Portland State University

PDXScholar

1996

\title{
An Evolutionary Simulation of the Tragedy of the Commons
}

Gretchen Oosterhout

Portland State University

Follow this and additional works at: https://pdxscholar.library.pdx.edu/open_access_etds Let us know how access to this document benefits you.

Recommended Citation

Oosterhout, Gretchen, "An Evolutionary Simulation of the Tragedy of the Commons" (1996). Dissertations and Theses. Paper 1251.

https://doi.org/10.15760/etd.1250

This Dissertation is brought to you for free and open access. It has been accepted for inclusion in Dissertations and Theses by an authorized administrator of PDXScholar. Please contact us if we can make this document more accessible: pdxscholar@pdx.edu. 


\section{INFORMATION TO USERS}

This manuscript has been reproduced from the microfilm master. UMI films the text directly from the original or copy submitted. Thus, some thesis and dissertation copies are in typewriter face, while others may be from any type of computer printer.

The quality of this reproduction is dependent upon the quality of the copy submitted. Broken or indistinct print, colored or poor quality illustrations and photographs, print bleedthrough, substandard margins, and improper alignment can adversely affect reproduction.

In the unlikely event that the author did not send UMI a complete manuscript and there are missing pages, these will be noted. Also, if unauthorized copyright material had to be removed, a note will indicate the deletion.

Oversize materials (e.g., maps, drawings, charts) are reproduced by sectioning the original, beginning at the upper left-hand comer and continuing from left to right in equal sections with small overlaps. Each original is also photographed in one exposure and is included in reduced form at the back of the book.

Photographs included in the original manuscript have been reproduced xerographically in this copy. Higher quality 6" $x$ 9" black and white photographic prints are available for any photographs or illustrations appearing in this copy for an additional charge. Contact UMI directly to order.

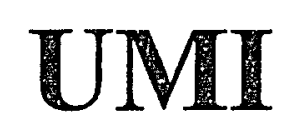

A Bell \& Howell Information Company 300 North Zeeb Road, Ann Artor MI 48106-1346 USA $313 / 761-4700 \quad 800 / 521-0600$ 


\title{
AN EVOLUTIONARY SIMULATION OF THE TRAGEDY OF THE COMMONS
}

by

\author{
GRETCHEN OOSTERHOUT
}

A dissertation submitted in partial fulfillment of the requirements for the degree of DOCTOR OF PHILOSOPHY

in

\section{SYSTEMS SCIENCE}

Portland State University

1996 
UMI Number: 9626732

UMI Microform 9626732

Copyright 1996, by UMI Company. All rights reserved.

This microform edition is protected against unauthorized copying under Title 17, United States Code.

\section{UMI \\ 300 North Zeeb Road \\ Ann Arbor, MI 48103}

Reproduced with permission of the copyright owner. Further reproduction prohibited without permission. 


\section{DISSERTATION APPROVAL}

The abstract and dissertation of Gretchen Oosterhout for the Doctor of Philosophy in Systems Science were presented April 10, 1996, and accepted by the dissertation committee and the doctoral program.

COMMITTEE APPROVALS:

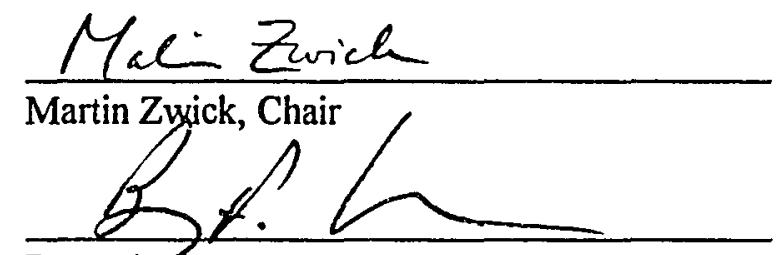

Barry Anderson

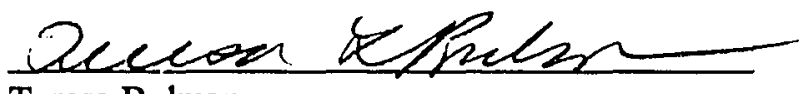

Teresa Bulman
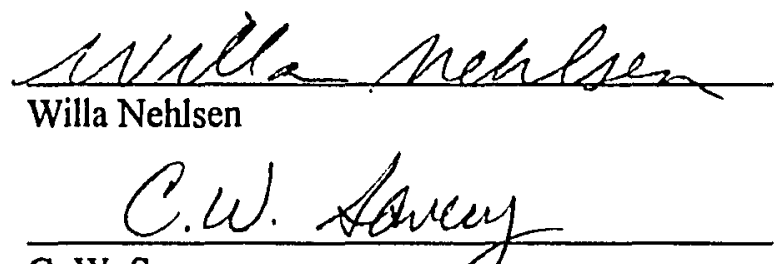

C. W. Savery

Representative of the Offico of Graduate Studies

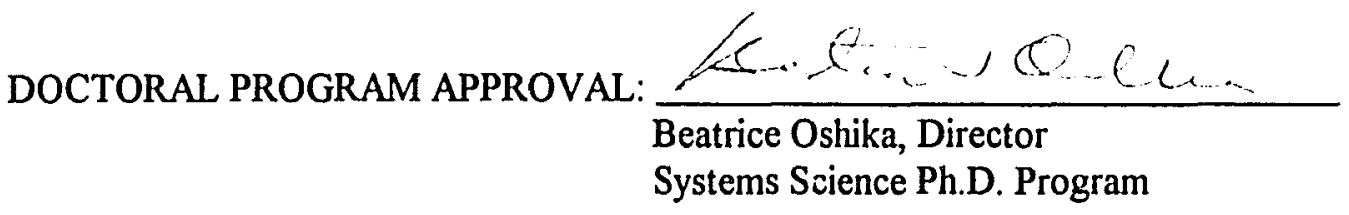

ACCEPTED FOR PORTLAND STATE UNIVERSITY BY THE LIBRARY

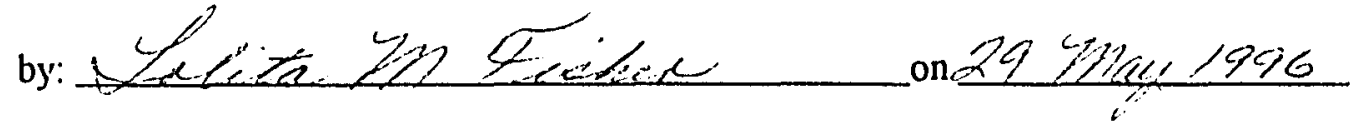




\begin{abstract}
A B S T RA C T
An abstract of the dissertation of Gretchen Oosterhout for the Doctor of Philosophy in Systems Science presented April 10, 1996.
\end{abstract}

Title: An Evolutionary Simulation of the Tragedy of the Commons

In his seminal essay, "The Tragedy of the Commons" (1968), Garrett Hardin argued that unless human population growth is controlled, the tragedy of common resource destruction is inevitable. This research consists of the development of an evolutionary computer model to simulate the Tragedy of the Commons, and social and economic solutions that have been proposed. In the simulations, multiattribute decision models are used to represent the tradeoffs a variety of types of individuals make among economic and social values in an uncertain environment. Individuals in each iteration of the simulation decide whether or not to exploit a common resource that has a stochastic regeneration rate. A genetic algorithm is used to simulate the way the decision makers respond to economic and social payoffs that result from their choices, as the commons responds to their actions over time.

Game theory analyses of the commons dilemma are also included that, in contrast to previous analyses of the Tragedy of the Commons, incorporate not only economic attributes, but social and aesthetic attributes as well. These analyses indicate 
that the games underlying the Tragedy of the Commons may be similar to not only the $\mathrm{N}$-person Prisoner's Dilemma, as is sometimes argued, but also $\mathrm{N}$-person games of Chicken, Benevolent Chicken, and Hero. Population diversity is found to be particularly important to solutions in both the evolutionary simulations and the game theory analyses.

The simulations and analyses support the hypothesis that, even if potential solutions that Hardin dismissed as unrealistic in the real world are given an opportunity to work in a simulated computer world, Hardin is right: for any given commons regeneration rate, the ultimate destruction of the commons can be prevented only by draconian economic or political measures, unrealistic rates of technological innovation or changes in social values, or coercive control of population growth. 


\section{CONTENTS}

List of symbols and abbreviations............................

List of Figures.....................................................iv

List of Tables...........................................................

List of Equations.....................................................viii

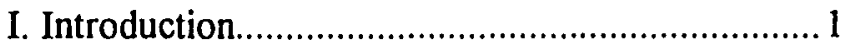

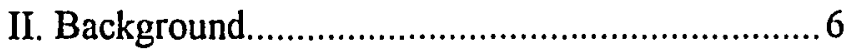

III. Game theory models vs decision theory models.. 19

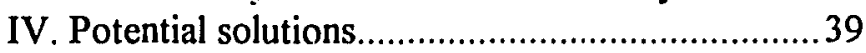

V. Significance of work.........................................66

VI. Modeling methodology .................................. 84

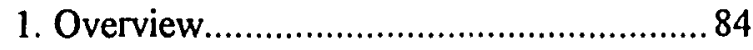

2. The commons model.............................. 93

3. The genetic algorithm............................ 94

4. The village model................................... 99

5. The decision model.................................. 100

6. Fitness..................................................... 131

7. Mathematical summary of model.............. 140

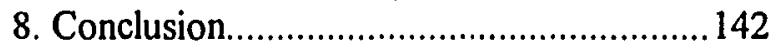

VII. Model development........................................ 144

Phase I: model development...................... 144

Phase II: factorial experiments..................... 154

VIII. Phase III: Potential solutions.......................... 177

Game theory analysis................................. 177

Solution simulations.....................................206

IX. Conclusion........................................................... 254

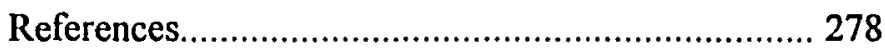

Appendix: Computer code listing............................ 285 


\section{SYMBOLS AND ABBREVIATIONS}

\begin{tabular}{|c|c|}
\hline$\hat{b}$ & Normalized regression coefficient \\
\hline Basin & Basin of attraction, type of longterm commons behavior pattern \\
\hline $\mathrm{BC}$ & Game of Benevolent Chicken \\
\hline$C_{\max }$ & Maximum conscience payoff \\
\hline DM & Decision maker; alternatively, Decision Model \\
\hline EMAV & Expected multiattribute value \\
\hline$E V$ & Expected value \\
\hline $\mathrm{EV}\{\}$ & Expectation operator \\
\hline$f$ & Final value \\
\hline fo & Generalized function of () \\
\hline$g_{0}$ & Gene that determines optimism or pessimism \\
\hline$g_{l}$ & Gene that determines coefficient of conscience payoff for Taking \\
\hline$g_{2}$ & Gene that determines coefficient of conscience payoff for Refraining \\
\hline$G$ & DM population growth constant \\
\hline GA & Genetic algorithm \\
\hline$i$ & Iteration number \\
\hline Iters & Iterations to extinction, dependent variable in factorial experiments \\
\hline ITQ & Individual Transferable Quota \\
\hline$j$ & Decision Maker genotype or Type number \\
\hline$k$ & Profit function parameter, determines slope and $y$-intercept \\
\hline$K$ & Commons maximum value, "carrying capacity" of $x$ \\
\hline$k_{f}$ & Final value for marginal profit function slope $k$ \\
\hline$k_{0}$ & Constant value for marginal profit function slope $k$ \\
\hline$l$ & $\begin{array}{l}\text { Outcome (e.g., DM Takes or Refrains, others Take or Refrain, and } \\
\text { commons is Good or Bad) }\end{array}$ \\
\hline$m$ & "Conscience" function parameter, determines slope and $y$-intercept \\
\hline MaxTakers & Maximum allowable harvest \\
\hline$m r$ & Mutation rate \\
\hline$M S E$ & Mean square error \\
\hline MSY & Maximum sustainable yield \\
\hline$M V L$ & $\begin{array}{l}\text { Minimum Viable Level, the value for } x \text { below which the commons } \\
\text { cannot recover }\end{array}$ \\
\hline$n$ & Slope of nonconsumable value function $N(x)$ \\
\hline$N\{\}$ & Normal distribution \\
\hline$N(x)$ & Nonconsumable value function \\
\hline$N_{\max }$ & Maximum possible value for nonconsumable value function \\
\hline$p$ & Probability \\
\hline $\mathrm{PD}$ & Prisoner's Dilemma game \\
\hline$p L$ & Probability of being among the legal first MaxTakers in a fishing de \\
\hline
\end{tabular}


$\mathrm{Pr} \quad$ Probability of being selected as a progenitor genotype to next iteration

$r_{0} \quad$ Commons regeneration rate constant

Refrainer DM that decides to Refrain from taking a unit of commons

$R V$

Realized Value

$r(x)$

Nominal Commons regeneration rate, $d x / d t$

SMV

Subjective marginal value

Stoch

$t$

$T \quad$ Number of Takers

Coefficient of variation of the stochastic regeneration rate: the standard

deviation divided by the mean

$T_{0} \quad$ Initial number of potential fishers

$T_{\max } \quad$ Total number of potential fishers

Taker DM that decides to Take a unit of commons

TFT Tit-for-Tat strategy for playing the PD

TSProb Probability that the higher-fitness member of a randomly selected pair of genotypes from one iteration will be selected for copying to the progenitor pool for the next iteration

Type $\quad$ Type of DM model, ranging from Hardin herdsman (Type I) to pure

Refrainer (Type IV)

$\bar{V} \quad$ Aggregate value function

$V_{c} \quad$ Normalized conscience function

$V_{\pi} \quad$ Normalized profit function

$w_{\pi} \quad$ Coefficient for the profit function

$x \quad$ State of the commons, in number of commons units

$x_{\infty} \quad$ Long term state of the conmons, at equilibrium

$z \quad$ Choice (Refrain or Take)

\section{Greek symbols}

$\alpha \quad$ Constant required to normalize the profit function

$\beta \quad$ Constant required to normalize the profit function

$\gamma \quad$ Rate of decrease of the subjective value function $V(\pi)$, reflecting

$\pi \quad$ Profit function

$\pi_{\max }^{\prime} \quad$ Marginal profit function maximum value; $y$-intercept in marginal profit

function graphs

$\pi^{\prime}(\mathrm{T}) \quad$ Marginal profit function

$\Delta t \quad$ Incremental time unit of discrete simultion

$\tau \quad 1$ for $z=$ Take; 0 for $z=$ Refrain 
Subscripts and superscripts

$0 \quad$ Initial or constant value

$c \quad$ Conscience function

$f \quad$ Final value

$i \quad$ Iteration number

$j \quad$ Decision Maker genotype or Type number

$l \quad$ Outcome (e.g., DM Takes or Refrains, others Take or Refrain, and commons is Good or Bad)

$n \quad$ Number of possible outcomes

$z \quad$ Choice (Take or Refrain)

* (Superscript) Particular value for number of Takers T or state of commons $x$ 


\section{LIST OF FIGURES}

FIGURE

Figure 2.1. Prisoner's Dilemma.

Figure 3.1. Game theory representation of the TOC.

Figure 3.2. Influence diagram of the TOC....

Figure 3.3. Prisoner's Dilemma and Chicken.

Figure 3.4. General case for payoffs of PD and Chicken.

Figure 6.1. Flowchart of the four interacting model components

of the TOC simulation.

Figure 6.2. Nominal commons regeneration rate curve, for $r_{0}=$ 0.04 ......

Figure 6.3. Marginal profit as a linear function of supply.

Figure 6.4. Marginal profit as a linear function of supply, at two population sizes, with constant slope.

Figure 6.5. Marginal profit as a linear function of supply, at two population sizes, with maximum marginal price held constant

Figure 6.6. Subjective marginal profit function (dotted line) and objective value function (solid line)

Figure 6.7. "Conscience" functions.

Figure 6.8. Type I or hard core Taker's decision tree

Figure 6.9. Type IV or hard core Refrainer's decision tree.

Figure 7.1. Hardin's TOC where the population is held constant, DMs do not take "Conscience" into account, and the commons is moderately stochastic (coefficient of variation $=2.0$ )

Figure 7.2. Hardin's TOC where the population, made up only of Type I DMs, grows at $G=0.0015$ per time increment

Figure 7.3. "Conscience" prevents the destruction of the commons, for a moderate, maximum population of potential fishers of twice the initial sustainable size (Figure 7.1)

Figure 7.4. "Conscience" solution fails for unlimited population growth.

Figure 7.5. Simulation with low population diversity on both diversity parameters: TSProb is 0.9 (low progenitor diversity) and $m r$ is 0.001 (low mutation rate).

Figure 7.6. Simulation with high progenitor diversity (TSProb is 0.5 ) but low mutation rate ( $m r$ is 0.1 ).

Figure 7.7. Simulation with high population diversity on both diversity parameters: TSProb is 0.5 (high progenitor diversity) and $m r$ is 0.1 (high mutation rate).

Figure 7.8. Simulation with high population diversity on both diversity parameters: TSProb is 0.5 (high progenitor diversity) and $m r$ is 0.1 (high mutation rate) (the same as the simulation in Figure 7.7), but with high stochasticity (Stoch $=2.0)$.

Figure 7.9. Interaction plot for stochasticity of the commons

Stoch and mutation rate $m r$, illustrating how low stochasticity and
PAGE

7

21

25

30

30

86

93

102

105

106

109

111

122

129

148

149

152

165

166

167 
high mutation rate contribute an effect that is greater than the sum of the two.

Figure 7.10. Interaction plot for TSProb and $m r$, illustrating a "crossing" interaction that can tend to hide single-factor effects.

Figure 7.11. Interaction plot for TSProb and Stoch, showing how when TSProb is high (diversity is low), the beneficial effect of low Stoch is canceled out.

Figure 7.12. TSProb*m interaction, illustrating the somewhatcrossing interaction between the parameter controlling most of the DM population diversity TSProb, and the parameter that determines the magnitude and slope of the "Conscience" function $m$.....

Figure 8.1. Two-person PD and Chicken, showing ordinal payoffs to row player (where " 3 " is best and " 0 " is worst)...

Figure 8.2. Payoff graphs of Prisoner's Dilemma and

Chicken.

Figure 8.3. Payoff graphs and two-person games of Hero and Benevolent Chicken.

Figure 8.4. Simplified decision tree.

Figure 8.5. Decision tree including nonconsumable use value $N(x)$

Figure 8.6. Base case payoffs for Type III DMs in the TOC

Figure 8.7. Type III payoff graphs, including an appropriate $N(x)$, showing Chicken-like preference ordering $(c>a>b>d) \ldots$

Figure 8.8. Type II payoff graphs, illustrating PD-like graphs when the commons is in good shape $(x=9800)$ but Chicken-like graphs when the commons is in bad shape $(x=2500)$.

Figure 8.9. Payoff graphs for Type I DMs, including $N(x)$

Figure 8.10. Summary of payoff graph game types, for Type II and III DMs.

Figure 8.11. TOC with population growth $G$ at 0.001 per iteration, profit function paramter $k$ of 3.0 , and "Conscience" function parameter $m=0.02$

Figure 8.12. The baseline simulation (Figure 8.11), but with demand parameter $k$ decreased by half. The commons survives longer than before, but still is harvested to extinction.

Figure 8.13. Simulation results when the profit function parameter $k$ is decreased by $10 \%$ if the commons falls below $x_{\text {max }} / 4$

Figure 8.14. Baseline simulation (Figure 8.11), but with a $20 \%$ increase in $r 0$ whenever the commons falls below $x_{\max } / 4$.

Figure 8.15. Baseline simulation (Figure 8.14), but with a more stochastic commons (Stoch $=1.0$, compared to 0.8 in previous examples).

Figure 8.16. Simulation results for increasing "Conscience" function paramter $m$ by $10 \%$ whenever the commons falls below $x_{\max } / 4$ 
Figure 8.17. Game between conservationist and extractor pressure groups, showing ordinal payoffs where the maximum $=3$ and the minimum $=0$.

Figure 8.18. Examples of linear and potential exponential forms of the "Conscience" function $C(x)$

Figure 8.19. Reference simulation, with $m=0.02, k=3.0$, and no $N(x)$

Figure 8.20. Population distribution in reference simulation (Figure 8.19), shortly before the commons goes extinct (Iter 100; $x=1380$ ).

Figure 8.21. Simulation identical to reference simulation (Figure 8.19), but with $N(x)$

Figure 8.22. Population distribution at Iter $=100$, with $N(x)$

Figure 8.23. Population distribution, just before the commons is destroyed $(x=260)$

Figure 8.24. Decision tree for fishers in a "free for all" fishery subjected to catch limits and potential penalties.

Figure 8.25. Typical results of simulations where the harvest levels are set at MaxTakers, and the perceived probability of getting caught for harvesting illegally is PrCaught. 


\section{LIST OF TABLES}

TABLE

PAGE

Table I. The four DM payoff Types and their payoffs

Table II. The four DM payoff Types and the alleles for the two genes $g_{l}$ and $g_{2}$. The alleles correspond to the coefficients of $C(x)$ in Table I

Table III. Summary table illustrating the total payoffs $\bar{V}$ that result from Eq. (6.14)

Table IV. Probability estimates and outcomes $\left[T^{*}, x^{*}\right]$ assumed by optimists and pessimists in the simulations

Table V. Settings for fifth factorial experiment

Table VI. Regression and ANOVA for the response variable indicating number of iterations to extinction Iter.

Table VII. Solution categories, methods for simulating, and brief explanations.

Table VIII. The four DM payoff types and their payoffs

Table LX. Contrasting contributions of the game-theoretical analysis and the decision-theoretical simulations. 


\section{LIST OF EQUATIONS}

(3.1) $\quad c>a>d>b \quad$ (Prisoner's Dilemma)

(3.2) $\mathrm{c}>\mathrm{a}>\mathrm{b}>\mathrm{d}$ (Chicken)

(6.1) $r(x)=\frac{d x(x)}{d t}=r_{0} x-r_{0} x^{2} / K$

(6.2) $r\left(x_{i}\right)=r_{0} x_{i}-r_{0} x_{i}^{2} / K$

(6.3) $x_{i+1}=x_{i}-T_{i}+N\left\{\left(r\left(x_{i}\right)\right.\right.$, Stoch $\left.* r\left(x_{i}\right)\right\}$

(6.4) $\operatorname{Pop}(t)=P o p(0) * \exp (G t)$

(6.5) $T \max _{i+1}=\operatorname{Tmax}_{i}+\operatorname{Tmax}_{i} * \exp (G \Delta t)$

(6.6) $\pi^{\prime}(T)=\pi_{\max }^{\prime}\left(1-T / T_{\max }\right)$

(6.7) $\pi^{\prime}(T)=\pi_{\max }^{\prime}\left(1-T /\right.$ Pop $\left._{\max }\right)$

(6.8) $\pi^{\prime}(T)=k_{0}\left(T_{\max }-7\right)$

(6.9) $\pi^{\prime}(T)=\pi_{\text {max }_{0}}\left(1-T / T_{\max }\right)$

(6.10) $\operatorname{SMV}\left(\pi^{\prime}\right)=\alpha-\beta\left(\exp \left(-\gamma \pi^{\prime}\right)\right)$

(6.11) $C^{\prime}(x)=-m x+C_{\text {max }}^{\prime}$

(6.12) $C^{\prime \prime}(x)=m(K-x)$

(6.13) $V_{c}\left(x_{i}, j, z\right)=\left[g_{1}^{j} C\left(x_{i}\right)\right] z+\left[g_{2}^{j} C\left(x_{i}\right)\right]\left(g_{2}^{j}-z\right)$

(6.14) $\bar{V}\left(T_{i}, x_{i}, j, z\right)=\left[w_{n}^{j} \pi\left(T_{i}\right)+g_{1}^{j} C\left(x_{i}\right)\right] z+\left[g_{2}^{j} C\left(x_{i}\right)\right]\left(g_{2}^{j}-z\right)$

(6.15) $\operatorname{EV}\left(T^{*}, x^{*}, j, z\right)=\sum_{x^{*}} \sum_{T^{*}} p\left(x^{*} \mid T^{*}\right) p(T \mid z) \bar{V}\left(T^{*}, x^{*} j, z\right)$

(6.16) $E V_{l}=\operatorname{Pr}($ Others Take $) * \operatorname{Pr}($ Commons Good Others Take $) *\left(w_{\pi}^{*} V_{\pi}+\right.$ $\left.w_{c}^{*} V_{c}\right)$

(6.17a) $\bar{V}\left(T_{i}, x_{i}, I I I, 1\right)=\pi\left(T_{i}\right)-C\left(x_{i}\right) \quad$ for Taking: $z=1$

(6.17b) $\bar{V}\left(T_{i}, x_{i}, I I, 0\right)=C\left(x_{i}\right) \quad$ for Refraining: $z=0$

(6.18) $F_{i j}=R V_{i j}=\bar{V}\left(T_{i}, x_{i}, j, z\right)$

(6.19) $\operatorname{Pr}(j, i+1)=f\left[\right.$ TSProb, $\left(F_{i j} / \frac{1}{T_{\max }} \sum_{j} F_{i j}\right]$

(7.1) $r(x)=r_{0} x-r_{0} x^{2} / K$ 
(7.2) $\quad \bar{V}_{I I I}($ Take $)=w_{\pi} \pi(T)-w_{c} C(x)=\bar{V}_{l l}($ Refrain $)=C(x)$

$\pi(100)=2 C(x)$

$w_{x}=w_{c}=1.0$

$k\left(T_{\max }-T\right)=2 m(K-x) \quad$ (from Eq. 6.8 and 6.12)

$3.0(200-100)=2 m(5000)$

(8.1) $\bar{V}($ Refrain $)=(a-b) R+b T_{\max }-a$

$\bar{V}($ Take $)=(c-d) R+d T_{\max }-d$

(8.2) $N(x)=n x+N_{\max }$

$n=\frac{N_{\max }}{x_{\max }}$

(8.3) $E V\{N(x)$, Take $\}=E V\{N(x)$, Refrain $\}=p w_{n} N($ good $)+(I-p) w_{n} N($ bad $)$

(8.4) $\pi(f e w)-C\left(x_{R}\right)+N\left(x_{R}\right)>C\left(x_{R}\right)+N\left(x_{R}\right)>$

$$
\pi(m a n y)-C\left(x_{T}\right)+N\left(x_{T}\right)>C\left(x_{T}\right)+N\left(x_{T}\right)
$$

(8.5) $\pi(\operatorname{many})-C\left(x_{T}\right)>C\left(x_{T}\right)$

(8.6) $\bar{V}_{\text {III }}($ Take $)=\pi(T)-C(x-T)+N(x-T)$

$\bar{V}_{I I I}($ Refrain $)=C(x-T)+N(x-T)$

(8.7) $C\left(x_{R}\right)+N\left(x_{R}\right)>\pi(m a n y)-C\left(x_{T}\right)+N\left(x_{T}\right) \quad$ or:

$$
C\left(x_{R}\right)+C\left(x_{T}\right)>\pi(m a n y)+N\left(x_{T}\right)-N\left(x_{R}\right)
$$

(8.8) $\pi(f e w)>2 C\left(x_{R}\right)$

(8.9) $C\left(x_{R}\right)+N\left(x_{R}\right)>C\left(x_{T}\right)+N\left(x_{T}\right)$ or:

$$
N\left(x_{R}\right)-N\left(x_{T}\right)>C\left(x_{T}\right)-C\left(x_{R}\right)
$$

(8.10) $N\left(x_{R}\right)-N\left(x_{T}\right)>C\left(x_{T}\right)-C\left(x_{R}\right)$

$$
\begin{aligned}
& \frac{d N}{d x}>-\frac{d C}{d x} \\
& n>-m
\end{aligned}
$$




\section{Chapter I}

\section{N T R O D U C T I O N}

This dissertation consists of the development of a computer simulation model that is used to explore the effects of individual decision processes on the development of, and potential solutions to, the Tragedy of the Commons (TOC). Garrett Hardin argued in his seminal 1968 essay, "The Tragedy of the Commons", that the tragedy of common resource destruction is the inevitable result in a finite world with no restrictions on population growth (Hardin, 1968). A "commons" was a public grazing area owned communally in villages in pre-industrial England and America, but the idea of a commons includes any resource (natural or otherwise) to which access is more or less open to all. Hardin argued that each villager always stands to profit by adding an additional cow to the commons, because the cost of the cow's grazing is shared by all, whereas the profit from the cow accrues to the villager alone. Villagers who decide not to add a cow in order to help conserve the commons stand to gain nothing, while their self-restraint adds to the profit earned by their less-responsible peers. Hence, the only "rational" decision is to exploit the resource.

Unfortunately, since all "rational" villagers must follow this line of reasoning, 
the result is that "ruin is the destination towards which all men rush, each pursuing his own best interest in a society that believes in the freedom of the commons" (Hardin, 1968). Hardin cites examples of such tragedies in the making as overgrazing of public lands and overfishing of the ocean. Hardin argues that as long as the population of exploiters is small enough that the resource can regenerate itself, the commons can survive; but because population growth ultimately increases demand beyond the commons' regeneration capacity, the tragedy of Malthusian "misery and vice" will inevitably result as necessary resources fall short of the requirements of the growing population.

Hardin argues that this tragedy is unavoidable because "natural selection favors the forces of psychological denial. The individual benefits as an individual from his ability to deny the truth even though society as a whole, of which he is a part, suffers" (Hardin, 1968). People who refrain from exploiting common resources, he says, have fewer viable offspring than those who exploit, and so biological and/or social evolution removes their sense of responsibility from the gene pool.

Hardin's model is driven by a neoclassical economist's strict definition of rationality, in which people who are "rational" choose behaviors that they believe will provide them as individuals the most economic benefit. In the model developed in this research, this definition of rationality is expanded to include other considerations; in particular, the decision-makers' consciences. In this model, a common resource is exploited by decision-makers (DMs) whose beliefs, values, and choices are represented 
by multi-attribute value models (Keeney and Raiffa, 1976; von Winterfeldt and Edwards, 1986). The social evolution of these DMs' beliefs and values is driven by how successful they are in achieving what they desire from the commons.

The commons is chaiacterized by its regeneration rate, which is affected not only by the actions of the DMs but also by stochastic natural disturbances (beneficial as well as harmful). Social evolution is modeled via a genetic algorithm (GA). GAs are computational methods based on genetics and evolution that are used for two, somewhat overlapping, purposes: (1) to solve nonlinear optimization problems, where the objective function (i.e., a mathematical function being maximized or minimized) is interpreted as a measure of relative "fitness" of potential optimization solutions; and (2) to simulate the genetic components of evolution. When used as an optimization tool, a GA is used to identify a region in the solution space that is relatively more optimal than other potential solution regions. When used as a tool for simulating evolutionary processes, a GA is used for simulating evolution, in order to explore evolutionary phenomena such as population dynamics (Lindgren, 1990), adaptation to environments (Bedau and Packard, 1990), interactions of hosts and parasites (Hillis, 1990) and punctuated equilibria (Lindgren, 1990). When a GA is used for optimization, the process by which the system evolves is generally of little interest; but when the GA is used as a tool to investigate evolution, the process itself and the final outcome are both of interest. In this research, the GA is used as a tool for simulating an evolutionary social process, rather than for doing an optimization. 
The GA is used here to explore how individual DMs' beliefs and values could evolve and interact, given a particular model of a commons and a variety of kinds of DMs. The purpose of this research is to explore approaches that have been proposed for solving the TOC, and to provide new insights into this important, and complex, kind of problems.

The fundamental research question is: "Is Hardin right?" That is, can the Tragedy of the Commons be avoided without controlling population growth?

In answering this question, the objectives are:

- To develop an evolutionary computer model that can be used to shed new light on the Tragedy of the Commons.

- To investigate what variables have the most impact on the outcome, and how they interact with one another.

- To investigate conditions under which the Tragedy of the Commons might be avoided.

The first five Chapters of this dissertation constitute the background and problem formulation. Chapter II begins with a description of the TOC, and contrasts the approaches to understanding the TOC favored in the fields of psychology, economics, and ecology. Chapter III discusses some difficulties with previous approaches to modeling the TOC. Chapter IV describes proposals in the literature for solving the TOC. Chapter V discusses the significance of the proposed work. Chapter VI describes the methodology used for simulating the TOC, Chapter VII describes the 
model development, Chapter VIII discusses the game theory analysis and simulation results, and Chapter IX is a conclusion and summary. 


\section{Chapter I I}

\section{B A C K G R O U N D}

Some of the most important, and intractable, problems facing humankind are social dilemmas such as the TOC. Dawes describes social dilemmas as being "defined by two simple properties: (a) each individual receives a higher payoff for a socially defecting choice (e.g., having additional children, using all the energy available, polluting his or her neighbors [sic]) than for a socially cooperative choice, no matter what the other individuals in society do, but (b) all individuals are better off if all cooperate than if all defect" (Dawes, 1980, 169).

The most well known model of social dilemmas is the Prisoner's Dilemma (PD). The PD is a game theory model of cooperation and competition, illustrated by the dilemma of two suspected criminals shown in the game theory matrix of Figure 2.1.

In this game, two suspected criminals are offered the same deal by the police: betray your accomplice and get the minimum sentence while your accomplice gets the maximum sentence, or stay silent. If you stay silent and your accomplice talks, your accomplice gets the minimum sentence (the best outcome, "3," indicating ordinal, not 
cardinal, preferences) and you get the maximum (the worst outcome, "0"), but if you stay silent and your accomplice also stays silent, you both get a reduced sentence. The reduced sentence is the second ("2") best outcome. The third (" 1 ") best outcome, a jail term longer than the reduced sentence but shorter than the maximum, results when both prisoners talk. For example, if $A$ is silent while $B$ talks, $A$ receives the worst ("0") outcome and $B$ receives the Best (" 3 ") outcome (Figure 2.1).

\begin{tabular}{|c|c|c|}
\hline & \multicolumn{2}{|c|}{ Prisoner B } \\
\hline & Silent & Talk \\
\hline Silent & 2,2 & 0,3 \\
\hline Talk & 3,0 & 1,1 \\
\hline
\end{tabular}

Figure 2.1. Prisioner's Dilemma. In each cell, the first payoff in each pair indicates the ordinal payoff to Prisoner $A$, and the second payoff indicates the ordinal payoff to Prisoner B. "3" indicates the most preferred outcome, "0" indicates the least preferred outcome.

The dilemma arises because both players have a dominant choice: both players prefer betraying their accomplice, regardless of what the accomplice does. The result is a deficient outcome: both receive the third-best outcome ("1, 1") when if they had somehow been able to cooperate and both stay silent, they would have been better off $(" 2,2 ")$.

The PD is a popular model of many social dilemmas because it so elegantly 
illustrates the conflict and apparent paradox between narrowly focused self-interest and the interests of the group to which a person belongs. For example, why should an individual ride a bus to work in order to reduce air pollution, since the payoff for being so responsible is the inconvenience and occasional frustration of relying on mass transit, with little real impact on the ultimate outcome? Taking a bus when others drive is the $(0,3)$ payoff in the PD. If many other people took the bus, everyone would enjoy cleaner air $(2,2)$, but it would still be more appealing to any individual to drive $(3,0)$. Driving is thus the dominant choice, with the deficient outcome of increased air pollution and traffic congestion $(1,1)$. The dilemma results because everyone's welfare would be improved if they could somehow reach a cooperative agreement to reduce driving $(2,2)$.

It is important to recognize that many people do take the bus to work, not only because they prefer riding to driving, but also because they enjoy a sense of doing the morally right thing. As a society, we particularly admire people who go so far as to give their lives for the sake of the morally right thing. Russian scientists chose to starve to death in the 1940's rather than eat the seeds in the world's largest seedbank, which had been left in their care by geneticist Nikolai Vavilov (Fowler and Mooney, 1990). Vavilov was arrested, and eventually died in prison, because genetics research was viewed as anti-communist, and because he dared disagree with the party line that acquired traits could be inherited. Before Vavilov's arrest he had managed to instill in his staff a belief in the vital importance of protecting biodiversity. A powerful belief it 
must have been, since they eventually died in order to protect their charge.

The point here is ihat the PD fails to predict the behaviors of a significant number of people, in a significant variety of settings, because some people consider different attributes and have different preferences than are accurately represented by the PD payoff matrix. Nonetheless, because it does so often predict what most people in fact do, it is a powerful model of the dilemma nature of many social and environmental problems.

Since the 1960s, there has been an explosion of research and literature concerning social dilemmas in the fields of psychology, political science, economics, biology, natural resource management, and ecology. Yet there is no consensus, even within fields, about how to solve these problems.

People who study human systems (social scientists), and those who study biological systems (ecologists) and have traditionally modeled social dilemmas from very different perspectives. Ecologists focus on biological systems, often relying on relatively simple models such as predator-prey relationships (e.g., Lotka-Volterra models, or Nicholson and Bailey's models (Holling, 1976)) or host-parasite models (Alexander, 1981). Their focus tends to be on characteristics of the biological system such as carrying capacity, species extinctions, and equilibrium concepts such as maximum sustainable yields. Because of all the difficulties in modeling these nonlinear systems, it has been difficult to develop ecosystem models unless stable equilibria could be assumed. Until recently, such systems have been analytically tractable only over 
small regions of the potential solution space, or through simplification so they could be treated as quasi-linear and stable. Furthermore, because the primarily biological perspective may miss important characteristics of the social, economic, and political system that cause social dilemmas, this perspective may also fail to get to the essence of the dilemmas.

In contrast to ecologists, social scientists have focused more on human behaviors such as organizational decision-making, community responses to resource scarcities, public policy development, and economic activities. Much theoretical and empirical work has been done in these fields, but researchers have had to rely on case studies, particularly in the fields of ocean fisheries (Hogarth, Norbury, Cunning, and Sommers, 1992; McCay, 1978; Ntiamoa-Baidu, 1991; Vrijenhoek, 1985), public lands management (Agarwall, Shukla, and Pal, 1993; Hardin and Baden, 1977), and water rights allocations (Allan and Flecker, 1993; Heinen and Low, 1992; Kompaore, 1989; Stott, 1991; Vrijenhoek, 1985); experimental work to learn more about why and how people respond to various aspects of dilemmas (Dawes, 1980; Edney and Harper, 1978; Vrijenhoek, 1985); theoretical arguments (Costanza, 1987; Dawes, 1980; Hamburger, 1973; Hardin, 1993; MacCrimmon and Messick, 1976); and very limited computer simulations such as iterated tournaments of the Prisoner's Dilemma (Axelrod, 1984; Axelrod, 1987; Hamburger, 1973; Lindgren, 1990; Miller, 1989). Just as few ecologists are social scientists, however, so are few social scientists ecologists. Ecologists and social scientists approach social dilemmas from within the 
frameworks of their particular fields, and neither field has been able to solve the problem. Political and economic solutions to social dilemmas are sabotaged by ecological aspects of the problem such as nonlinear, stochastic, or chaotic characteristics so common in biological systems but so uncommon in political, social, or economic models.

The contrast in perspectives is perhaps most dramatic between economists and ecologists. Although ecology and economics are beginning to share the rejection of reductionism in favor of a system level focus, as well as the acknowledgment of the fact that feedback cycles may be not only stabilizing but also amplifying (Hardin, 1993), there are some profoundly important differences. To begin with, economists focus on human economic systems, regarding the biologic system is primarily a source of raw materials and a sink for waste; while ecologists focus on biologic systems, regarding humans primarily as disturbance input sources.

In addition, although there is a growing understanding of nonlinear dynamic systems, chaos, and catastrophe theory, the assumption of ceteris paribus ("everything else being equal") is explicit in discussions of economic system response to perturbations, at least in most classical economics textbooks (Hardin, 1993). As a result, in a neo-classical economic system, nonlinearities tend to be ignored (Hardin, 1993). For this approach to be valid, the variables must be independent from one another and the system must be linear, or at least the effects of nonlinearities and interactions must be small. In contrast, the most fundamental idea of ecology is that 
everything is connected to everything else: "you can never do just one thing" (Hardin, 1993). Interactions are very important to ecologists: "As the ecologist might say, it is the secondary impacts that will get you if you do not consider the whole" (Odum, 1977, 1292).

The most fundamental construct of neo-classical economics has long been the "rational actor" model, which is based on the assumption that people left to their own devices will do what is in their own best interests. According to Adam Smith, the net effect of all these rational actors is that an "invisible hand" appears to guide their individual efforts to the serendipitous result that all prosper (Hardin, 1993). In contrast, according to ecologists such as Hardin (1993) and psychologists such as Dawes (1980), neo-classical economics is based on the fallacy of composition. That is, what appears to be good to an individual may end up being disastrous for society. This is particularly true when individuals are making decisions about commonly-held resources such as air, ground water, ocean fisheries, national forests, and public rangelands. As Hardin put it in his famous essay (1968, 20, ellipsis in original):

As a rational being, each herdsman seeks to maximize his gain. Explicitly or implicitly, more or less consciously, he asks, 'What is the utility to me of adding one more animal to my herd?' This utility has one negative and one positive component.

1. The positive component is a function of the increment of one animal. Since the herdsman receives all the proceeds from the sale of the additional animal, the positive utility is nearly +1 .

2. The negative component is a function of the additional overgrazing created by one more animal. Since, however, the effects of overgrazing are shared by all the herdsmen, the negative utility for any particular decision-making herdsman is only a fraction of -1 . 
Adding together the component partial utilities, the rational herdsman concludes that the only sensible course for him to pursue is to add another animal to his herd. And another...But this is the conclusion reached by each and every rational herdsman sharing a commons. Therein is the tragedy. Each man is locked into a system that compels him to increase his herd without limit - in a world that is limited.

Dawes suggests that we "contrast Hardin's analysis of herdsmen rushing toward their own destruction with Adam Smith's...analysis of the individual worker's unintended beneficence in a laissez-faire capitalistic society" (Dawes, 1980, 172). As Adam Smith put it $(1776,477)$ :

As every individual, therefore, endeavors as much as he can both to employ his capital in the support of domestic industry, and so to direct that industry that its produce may be of the greatest value; every individual necessarily labors to render the annual revenue of the society as great as he can...By preferring the support of domestic to that of foreign industry, he intends his own security; and by directing that industry in such a manner as its produce may be for the greatest value, he intends only his own gain, and he is in this, as in many other cases, led hy an invisible hand to promote an end which was no part of his intention.

The difference between Hardin and Smith, says Dawes, is not that they have different beliefs about the effects of selfish actors, but that "they are discussing different situations," in that Hardin is concerned with externalities that "are negative and greater than the individual's payoffs; [whereas] in Smith's Scotland they are positive" (Dawes, $1980,173)$. Both are describing nondeterministic, emergent systems, but in ecologist Hardin's system, the whole is greatly threatened, and ultimately diminished, by the sum of the parts. In economist Smith's system, the whole is greater than the sum of the 
parts.

Hardin and Smith are both describing systems that include positive-feedback cycles, but in Hardin's, feedback amplifies the rate of growth of harmful impacts, whereas in Smith's, feedback amplifies the rate of growth of beneficial impacts. In Hardin's system, social dilemmas tend to accelerate the destruction of the commons and ultimately the welfare of the citizens who are dependent on it. Smith's system, in contrast, depends for its very stability, and also its presumed beneficence, on social dilemmas: in a free market consisting of a large number of producers and consumers, there is always motivation for any member of a collective (e.g., a collective of productprice-setting producers, or labor-cost setting workers) to defect from the group's attempt to control the market. Smith and Hardin are not describing systems that are simple opposites; they are defining their systems differently and making different assumptions about stability: Smith treats as external to the problem the finiteness of resources that Hardin believes to be central.

Many researchers agree that a major cause of social dilemmas is the human tendency to discount the importance of potential impacts that seem spatially or temporally distant (Heinen and Low, 1992). They argue that the ability of humans to discount probably evolved in response to uncertainty, so that "the longer or farther something is away from our immediate reproductive interests, the less it will pay us to invest time, money, and/or energy, in its consideration" (Heinen and Low, 1992, 111). Animals and even insects apparently discount in much the same way (Griffin, 1992). 
Discounting fails to take into account the conflict regarding what the future will be like. Hardin portrays this conflict as being between the "meliorist" and the "pejorist" perspectives (Hardin and Baden, 1977, 126). Meliorists (such as many neo-classical economists, according to Hardin) believe that even if we truly are running out of fossil fuels and destroying the rain forests, the ozone layer, and so on (and they do not necessarily concede that we are), we will find a way to fix or ameliorate the problem (Morowitz, 1991; Pimm, Russell, Gittleman, and Brooks, 1995; Simon and Wildavsky, 1993). Pejorists, on the other hand, believe that all this meddling in extremely complex systems which we do not understand can result only in disaster, not only for human beings but also for the biosphere (Ehrenfeld, 1981; Hardin, 1992; Meffe and Ehrlich, 1993). Meliorists are optimistic, not only about whether we are creating disasters, but also about whether we can recover from them if they should happen to occur; whereas pejorists are pessimistic, believing that we are indeed creating disasters, and that they are not likely to be reversible.

Economists employ discounting explicitly by using interest rates to discount future costs and benefits in public policy decision-making. The result is that time frames greater than a decade or two simply drop out of economic decisions. Conservationists object, arguing that some environmentally important investments, such as growing forests or rebuilding watersheds, "may require 50 or more years to come to fruition" (Weiss, 1990, 30). Ecologist E.P. Odum suggests that a major source of conflict between conservation-oriented people and economics-oriented people is the difference 
in time frames considered. He says economic time frames tend to be two to four years because of the electoral cycle, in contrast to ecologists' time frames, which may be as long as centuries (Odum, 1977). Furthermore, the practice of using a discount rate in economic decision models encourages, rather than counteracts, the human tendency to disregard future effects, so that "the very process of discounting...encourages the public to underestimate the importance of future costs and defer their payment" (Daily and Ehrlich, 1992, 767).

Others object to discounting on moral grounds, because they see the conflict as between this generation and the next (Weiss, 1990). Some argue that discounting implies "...a gamble with the welfare of future generations, [because] estimates of future costs and benefits are uncertain, and there is both subjectivity and uncertainty in the selection of an appropriate discount rate" (Daily and Ehrlich, 1992, 767). Discounting may get carried to extremes when individuals expect that if they do not exploit a scarce resource now, someone else will; hence the future value of the resource to those individuals is essentially zero.

Hardin (1968) pointed out that distributional equity is theoretically an optimization problem. Different people have different value systems, so that even posing the optimization problem is difficult: one person might believe that immediate economic growth is the most important benefit not only to this generation but to future ones whose welfare is assumed to build on today's success. Another may believe that the future aesthetic values of streams and forests are most important. The TOC is 
enormously complicated by social values, and the question of how we are to compare the actual values that different people put on different things - "commensuring the incommensurables," as Hardin says, is difficult: "We want the maximum good per person; but what is good? To one person it is wilderness, to another it is ski lodges for thousands. To one it is estuaries to nourish ducks for hunters to shoot; to another it is factory land. Comparing one good with another is, we usually say, impossible because goods are incommensurable. Incommensurables cannot be compared". He offers an uncharacteristically optimistic view when he goes on to add (1968, 18-19):

...in real life incommensurables are commensurable. Only a criterion of judgment and a system of weighting are needed. In nature the criterion is survival. Is it better for a species to be small and hideable, or large and powerful? Natural selection commensurates the incommensurables. The compromise achieved depends on a natural weighting of the values of the variables...It is when the hidden decisions are made explicit that the arguments begin. The problem for the years ahead is to work out an acceptable theory of weighting. Synergistic effects, nonlinear variation, and difficulties in discounting the future make the intellectual problem difficult, but not (in principle) insoluble.

Unforturately, Hardin could not tell us exactly how we should go about determining — let alone agreeing on as a society — what the "criterion of judgment" and system of weighting should be. In fact, even if we could identify and agree on this theoretical objective function, it would undoubtedly be complex and nonlinear. There is no way to identify the global optimum for nonlinear optimization problems, although for many problems, the GA can at least identify regions of a solution space that are relatively more optimal that others. As Hardin acknowledges, the problem is indeed 
extremely difficult and may even be insoluble. Nonetheless the problem is important, and it is just these criteria of judgment, systems of weighting, and synergistic (nonlinear) effects, that this work explores. 


\section{Chapter II I}

\section{GAME THEORY MODELS VERSUS DECISION THEORY MODELS}

\section{A GAME THEORY MODEL AND A DECISION THEORY MODEL}

The two most widely used approaches to analyzing complex decisions are game theory and decision theory. There are advantages and disadvantages to both, and in order to see how these advantages and disadvantages might affect understanding the TOC, consider a simplified case of a DM who is considering whether to exploit a commons. The DM could be, for example, a logger who is deciding whether to undertake a clear-cut, a commercial fisher deciding whether to expand a fishing fleet, a factory owner deciding whether to pollute, or a couple deciding whether to have another child. We shall rely here on Hardin's idea of a herdsman and a common grazing area: a herdsman is deciding whether to add an additional cow to the open range. This herdsman is considering only two decision options (add a cow or not), 
although of course the decision could involve how many cows to add, whether to add them now or later, and so on.

There are many uncertainties facing anyone trying to make this kind of decision, and evaluating uncertainties is one of the most important parts of decision-making. However, for the sake of conceptual simplicity, all these uncertainties are here reduced to two potential outcomes: either everything will be "okay," or it will not. For example, the DM might ask whether the commons can carry an additional cow at a profitable level, and whether the market will be profitable when the cow is ready to be sold. There is the question of whether, if "everything" turns out not to be okay, the problem can be fixed or adapted to: that is, if the commons collapses, can the herdsman can go into another line of work, or can a technological fix be found, and so on. This decision could be conceptualized in a game theory matrix shown in Figure 3.1, where a herdsman is playing a game against Nature (the payoffs shown are the payoffs to the herdsman; in game theory, Nature receives no payoff but is only an actor).

In this matrix, the best outcome for the herdsman is when he adds a cow and everything is "okay" (cell "3" in Figure 3.1). The worst outcome ("0") is if he does not add a cow and everything is "not okay" anyway. In this matrix, the second ("2") best outcome is preferred to the third best outcome (" 1 ") because Hardin argued that a herdsman who adds a cow would still be better off than the herdsman who decides not to add a cow, regardless of whether everything turned out "okay" or not (Hardin, 1968). This point is certainly debatable, but for the moment we shall remain consistent 
with Hardin.

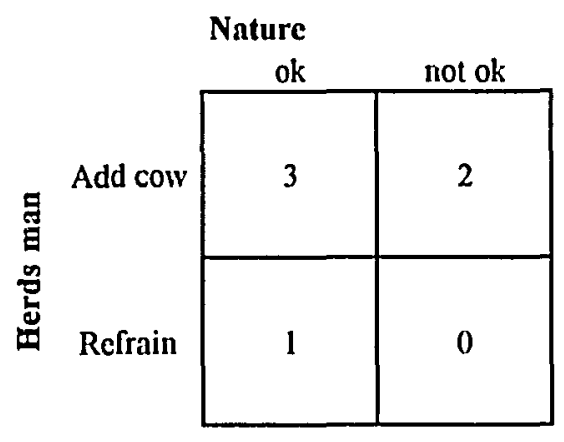

Figure 3.1. Game theory representation of the TOC. Ordinal payoffs $3,2,1$, $0=$ Best, Second, Third and Worst outcomes for the Herdsman.

The point illustrated by this simple matrix (for either arrangement of the "2" and "1" preferences) is that, according to the classic model, the herdsman has a strategy that is dominant; i.e., no matter what Nature does, he is always better off adding a cow. The reason for this is the key to the TOC as a social dilemma: the cost to the herdsman of any damage his added cow might do to the commons is small because it is shared by all, whereas the immediate benefit to him of adding a cow is potentially significant, because it is shared with no one. Regardless of whether or not the herdsman believes that "everything" will be "okay," as long as he believes that the costs will be mostly paid by others while the benefits will accrue only to him, he has no rational choice but to add a cow. In Hardin's terms, meliorists would assume the probability of "everything okay" to be high, regardless of which decision option they chose: hence, why not take the profitable option? Pejorists will see that "everything" may not be "okay", regardless of their choice. Since again it does not matter what they do, why not add the cow 
anyway?

The tragedy occurs because all herdsmen are supposedly "rational" and therefore must follow this same line of reasoning (a dominant choice), with the result that everyone decides to add an animal, and the commons gets destroyed (a deficient outcome). Whether or not any of the herdsmen recognizes the inescapable end result is irrelevant to their immediate decision regarding adding one more animal, because it is theoretically always rational to just add that one cow. This conflict between individual self interest and the long-term interests of the society to which the individual belongs is what results in the tragedy, because the individual's self interest always wins, and ultimately everyone loses.

The TOC in the real world is more complicated than a simple game theory model, however. Herdsmen may take into account more attributes than just their immediate self interests, and most important, uncertainty plays a substantial role in the real world. Although multiple attributes can be represented in game theory matrices, only the aggriegate payoffs can easily be shown in the cells. Illustrating the relative impacts of different attributes is thus awkward at best. The decision trees used in multiple attribute decision models show the individual attribute function payoffs clearly, and thus are more useful than game theory matrices for illustrating complex tradeoffs. Furthermore, one of the most important advantages of decision theory over game theory is that uncertainty is an important part of decision theory models, whereas game theory cannol represent probability notions about future states of nature. 
There are many theories about how people actually do make decisions.

However, it is widely accepted in decision theory that the normative decision rule is Expected Multiattribute Value or Utility (EMAV or EMAU), in which DMs try to choose the course of action that will maximize the value or utility they will receive as a result of their actions (von Winterfeldt and Edwards, 1986). One of the reasons EMAV is the normative choice is that it requires explicit consideration of all the important components of a decision (i.e., attributes, attribute weights, value functions, and risk attitudes in the EMAU part of the model, and beliefs about uncertain events in the expectation part of the model). Although the analytical rigor of EMAV lends itself well to computer modeling, it has not been used much outside the field of decision theory and optimization. Because the use of EMAV to model evolving decision processes is believed to be unique, this work should help expand the scope of applicability of this powerful tool. For these reasons, DMs in this model follow the EMAV rule (see Chapter VI).

It is important to note that there is little if any evidence that people ordinarily make decisions this carefully. We are known to be subject to a variety of biases, and to rely on sometimes-counter-productive heuristics that reduce the effort required to make decisions, but reduce their efficacy as well (c.f. Costanza, 1987; Russo and Shoemaker, 1989; von Winterfeldt and Edwards, 1986). Assuming that DMs use EMAV is a "best case" approach, which errs on the side of showing Hardin to be wrong. The burden of proof, in other words, remains with Hardin. 
To see how the decision problem shown in the game theory matrix. (Figure 3.1) might be modeled in more detail with EMAV, consider the influence diagram shown in Figure 3.2. The herdsman's choices that were shown in Figure 3.1 are the same in the influence diagram (shown lower case in parentheses), but Nature's choices are shown in the event nodes, which in an EMAV model have probabilities associated with them (event nodes that are completely determined by predecessor nodes shown in the influence diagram are indicated with rounded-corner boxes, whereas event nodes only partially determined by predecessor nodes are indicated with ovals). The decision choice is shown in the square box in Figure 3.2: TAKE OR NOT? Arrows indicate direction of influence. The net value of the outcome to the DM is indicated with the sideways triangle on the right side of Figure 3.2.

In Figure 3.2, PAYOFF TO ME is determined by two things: whether or not the DM chooses to TAKE, and the STATE OF THE COMMONS, which is in turn affected by ACTIONS BY OTHERS. The number of DMs who extract or preserve (ACTIONS BY OTHERS) affects the STATE OF COMMONS and ultimately a particular DM's payoffs. The STATE OF COMMONS node is shown as an oval rather than a rounded box because it is not completely determined by its predecessor node (ACTIONS BY OTHERS), but also by other factors not shown in the influence diagram (e.g., Nature's stochasticity). PAYOFF TO ME and PAYOFF TO OTHERS are both completely determined by their predecessor nodes, and are shown as rounded boxes. 


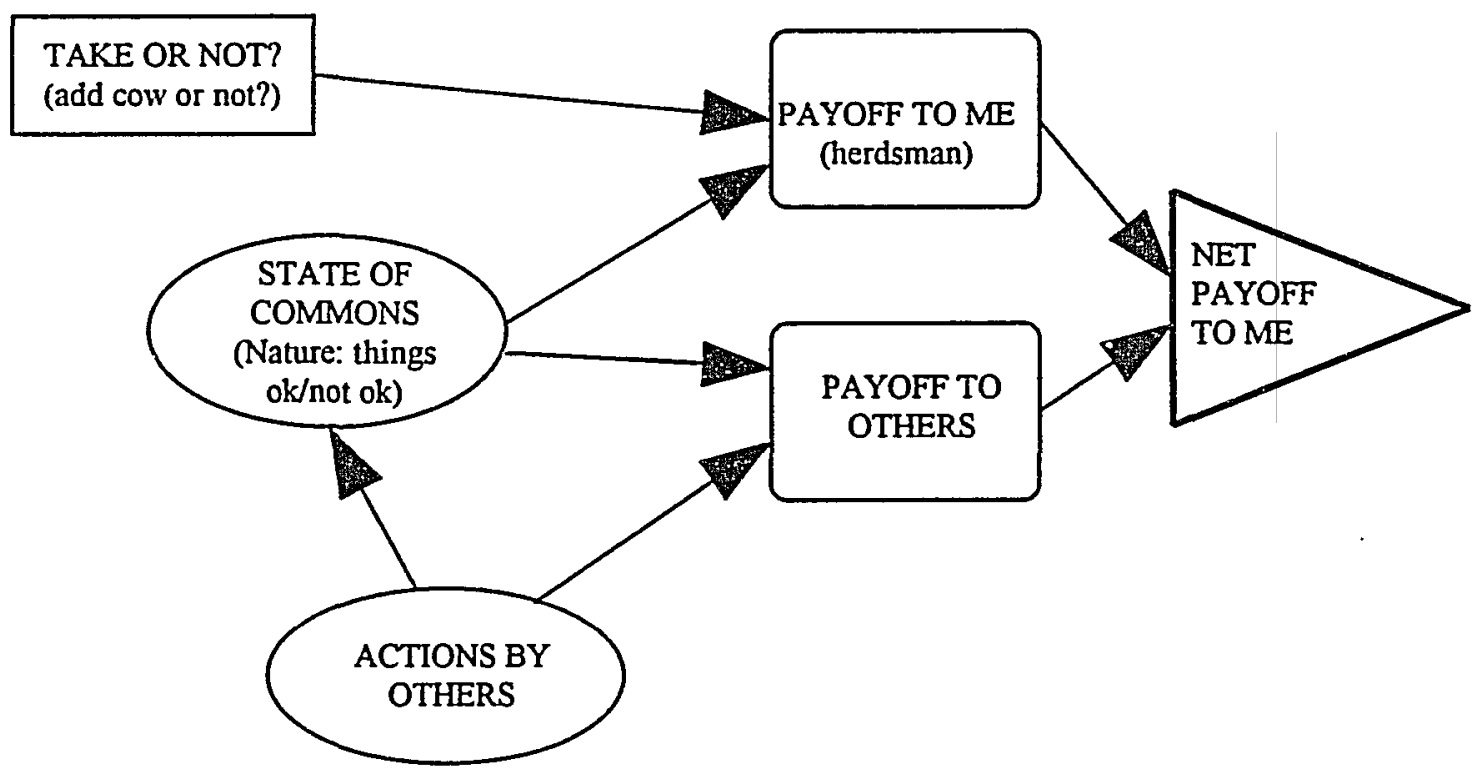

Figure 3.2. Influence diagram of the TOC. 
In this work (though not in Hardin's paper) PAYOFF TO ME may consist of not only a direct payoff due to a share of the commons taken or not (e.g., the money the herdsman gets from grazing a cow, or foregoes for not grazing a cow), but also a payoff that has to do with the DM's moral concerns, if any. The DM's sense of moral concerns affects how the DM feels about the course of action selected. The NET PAYOFF TO ME may thus consist of not only the DM's direct payoff from the commons, but also an indirect payoff due to that DM's moral concerns.

One of the key characteristics of the dilemma of the TOC is reflected in the fact that the decision made by any individual DM has negligible effect on the state of the commons: hence, there is no arrow from the decision nade to the STIATE OF COMMONS; but the aggregate effect from many other DMs does have significant effect on the state of the commons in each generation: hence, there are arrows between ACTIONS BY OTHERS and STATE OF COMMONS, Most of an individuals DM's actual payoffs (for a particular choice) is determined by what other DMs do.

The EMAV decision model is different from the game theory matrix in three key ivays: (1) in the EMAV model, uncertain events are incorporated into the calculation via probabilities, whereas in game theory, the likelihood of uncertain events is not indicated; (2) in EMAV, the sequence of events that could implact the outcome is clear, whereas in game theory it is not (although game "moves" by the players can be shown in extensive, or sequential, form); (3) in EMAV, the tradeoffs a DM might make among multiple attributes are explicit because the single-attribute value functions 
are important components of decision trees, whereas in game theory matrices, the tradeoffs among multiple attributes are not so clear because only the total payoffs are typically shown in the matrix cells.

\section{GAME THEORY MODELS}

The model developed in this dissertation may be the first computer model of social dilemmas based on the TOC. Many computer models of social dilemmas have been based on the Prisoner's Dilemma (Axelrod, 1984; Dawes, 1980; Hamburger, 1973; Lindgren, 1990), although it appears from the literature search that none has presented itself as a model of the TOC.

Because both the TOC and the PD are models of dilemmas where individually "rational" decision-making leads to collectively irrational outcomes, it could be argued that under some conditions the TOC could be modeled as an "N-person PD," which is a PD played by more than two players at a time (for a detailed description of $\mathrm{N}$-person game analysis methodology, see Chapter VIII). In the N-person PD, the payoff each player receives depends on how many players choose to remain loyal (in game theory terms, this option is to "Cooperate") and how many choose betrayal (in game theory terms, this option is to "Defect"). The underlying payoff structure is the same as in the pairwise PD, and is in effect summed over all players (see Chapter VIII).

The question here is whether the N-person PD is a good model for the TOC. 
The "remorseless logic" of Hardin's TOC (Hardin, 1968) is certainly present, because the collectively irrational result of the underlying two-person game is also characteristic of the N-person game. However, a number of researchers (Dawes, 1980; Hamburger, 1973; Rapoport, 1967; Shubik, 1970) have questioned the validity of applying the Nperson PD to social dilemmas. In particular, Hamburger (1973) explored N-person social dilemmas through the examination of two similar, but still distinct, types of games: games of contribution, and games of conservation. As an example of a "contribution problem" he describes a situation where each person in a group of people considers contributing a fixed sum to provide a playground that all can enjoy. Access to the playground is open to everyone, regardless of whether they contribute, and thus the "free rider" logic of the game dictates that no one contributes (a dominant choice). The result, of course, is that there ends up being no playground (a deficient outcome).

Hamburger argues that social dilemmas that arise because of conservation concerns, which he calls "conservation problems," are fundamentally different from "contribution problems" such as the playground problem described above. This distinction is important because it is argued here that the TOC is a conservation problem, not a contribution problem, and as such may not best be modeled as an Nperson PD. Hamburger says that a conservation problem occurs, for example, when residents of a city in the grip of a heatwave are warned not to run their air conditioners for fear of electrical blackout. He calls this a "probabilistic take-some game" (Hamburger, 1973). In this kind of game, "if everyone refrains, the resulting decrease in 
probability of blackout corresponds to an increased expected utility to the individual which exceeds his cost in perspiration.... Thus again, if each individual acts in his own best interest, the result is worse for each of them than if each had acted oppositely" (Hamburger, 1973, 28). He argues that, although the irrationality of the net result is typical of PDs, the games of contribution (e.g., the playground problem) and conservation (e.g., the blackout problem) are fundamentally different from each other. In short, he says the contribution problem becomes a game of the same form as an $\mathrm{N}$ person $\mathrm{PD}$, whereas the form of the game underlying the conservation problem depends on who is playing. Because this is a subtle but important point, Hamburger's arguments will be considered in more detail.

Hamburger argues that a different game than the PD could underlie the game of conservation: the game of Chicken. Chicken is based on the contest between two hotrodders racing towards each other in a single lane; the driver who "chickens out" by swerving out of the lane loses face but is not otherwise injured (or dead), whereas if neither chooses to chicken out, both may die in the resulting crash. A comparison of payoffs between the two games is shown in Figure 3.3, and a generalization of the two is shown in Figure 3.4.

If the TOC were posed as a game of Chicken instead of the PD, the worst outcome for any DM would no longer be to not add a cow to the commons when the other player (or players, in the case of an N-player game) added a cow (outcome $(0,3)$ in Figure 3.3a). In Chicken, the worst outcome for any player would be for both (all) 
players to add cows (outcome $(0,0)$ in Figure $3.3 \mathrm{~b}$ ), a point of view that may be more consistent with the environmentalist's or pejorist's perspective than the herdsman's.

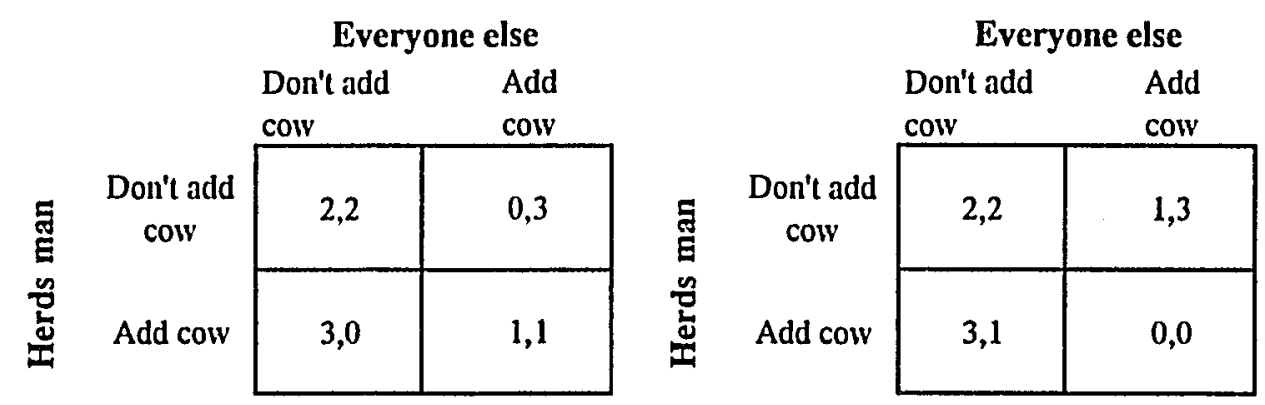

Figure 3.3a Prisoner's Dilemma

Figure 3.3b. Chicken

Figure 3.3. Prisoner's Dilemma and Chicken.

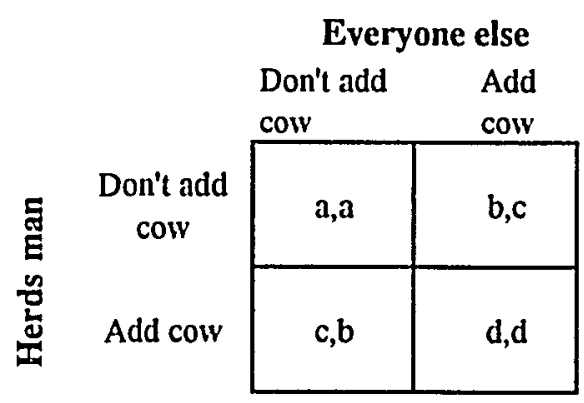

Figure 3.4. General case for payoffs of PD and Chicken.

In the PD shown in Figure 3.3a, the payoff preference order for a PD is:

(3.1) $c>a>d>b$

whereas in Chicken (Figure 3.3b), the preference order is:

(3.2) $c>a>b>d$

The difference between these two games is that the preference of $d>b$ of Eq.

(3.1) is reversed in Eq. (3.2): in the PD, the dominant strategy (shown shaded in Figure 
3.3) is always to add a cow, regardless of what the other player does, but in Chicken, there is no dominant strategy: the best choice depends on what the other player does.

Hamburger argues that whether a game of conservation is more like the PD or Chicken depends on the mix of strategies of the various players. That is, the strategic form of the game depends on how many players are deciding together at a time, how much they know about the other players' actions, what their preferences are, and what they believe about the true state of Nature. In the PD, none of that information affects the DM's choice, because defecting is always the dominant strategy; but in Chicken, the other players' likely actions do matter, regardless of whether the other player is other humans or Nature.

In a game of conservation, the city residents suffering from the heatwave have no way of knowing the point at which point the blackout will occur, and their payoff structures beyond that point are different from/their payoff structures just before that point. As long as the players are fairly certain that they are reasonably safe: from disaster, the game is probably most like the PD. But if any players believe they may be in the region of this disaster point, the game becomes more like Chicken to them. That is, the probabilities regarding what the other players, and Nature, will do, matter.

Different players may have different beliefs and values, and thus maly be playing different games and following different strategies. This situation cannot beirepresented with the $\mathrm{N}$-person $\mathrm{PD}$. If there are only relatively a few players, there is neither dilemma nor tragedy, since an individual's paydff for adding a cow is more /or less the 
same regardless of whether or not others add cows. If the commons has collapsed, adding a cow is pointless because it will starve to death: again there is no dilemma. The dilemma occurs when the commons is in the gray area where it is in danger of being exploited beyond its carrying capacity. Because Nature is playing a significant role, the true form of the underlying game is thus probabilistic: if Hamburger is right, then the TOC is in reality more like a game among $N$ players plus Nature (Hamburger, 1973), and under many of the most important circumstances is thus not well modeled by the N-player PD.

\section{PRISONER'S DILEMMA COMPUTER TOURNAMENTS}

Another approach to modeling N-person social dilemmas has been to allow a mix of PD strategies to compete with one another in a computer-simulated tournament. Axelrod (1984) held two such tournaments, in which each strategy submitted by a contestant played one at a time against all the other strategies submitted, each accumulating game points according to the two-person PD payoff matrix. Both tournaments were won by a simple strategy submitted by Amnon Rapoport, called "Tit for Tat" (TFT). In TFT, the player chooses the "Cooperate" option at the first encounter with another strategy, and thereafter chooses whatever action the opponent used in the last move. If two TFT players encounter each other repeatedly, they would consistently cooperate and would thus win the maximum number of points over time. 
Axelrod recruited biologist William Hamilton to help interpret the results of his tournaments in the light of evolutionary theory, and they developed an idea they called "cooperation theory" to extend the results of these tournaments to solving social dilemmas. He argued that these tournaments demonstrate that cooperation could evolve in a population if enough TFT players exist, because TFT is both "nice" and "provokable." By this he meant that a TFT player would generously offer to cooperate first, thereby running the chance of being taken advantage of by a "mean" strategy, but because a TFT player repleats the other player's previous move, a TFT player is "provokable" and thus can be taken advantage of only once. He concluded that the computer tournaments show that (Axelrod, 1984, 173):

...cooperation can get started by even a small cluster of individuals who are prepared to reciprocate cooperation, even in a world where no one else will cooperate. The analysis also shows that the two key requisites for cooperation to thrive are that the cooperation be based on reciprocity, and that the shadow of the future is important enough to make this reciprocity stable.

Axelrod emphasized that a social order based on reciprocity requires that individuals be able to recognize one another and to know the prior history of their interactions. He argued that since even bacteria could meet this requirement (by interacting with only one kind of organism), "so can people and nations" (Axelrod, $1984,173)$. He further claimed that his tournaments showed that "there is no need to assume trust between the players: the use of reciprocity can be enough to make defection unproductive. Altruism is not needed: successful strategies can elicit 
cooperation even from an egoist. Finally, no central authority is needed: cooperation based on reciprocity can be self-policing" (Axelrod, 1984, 174).

Artificial life researchers have also explored $\mathrm{N}$-person PD competitions, by modeling PD strategies as genotypes competing pairwise for game points in simulated evolutionary systems (Axelrod, 1987; Lindgren, 1990; Miller, 1989). Miller and Axelrod both used GAs to simulate pairwise competition among PD strategies. Miller allowed all the strategies to compete pairwise against one another, so that each strategy worked as a selection mechanism for each other strategy, whereas Axelrod used the higher-scoring performers from his previous iterated tournaments (Axelrod, 1984) to provide the fitness selection mechanism, although he too relied on pairwise interactions for the payoffs.

Kristian Lindgren developed the most intriguing evolutionary model of the PD because he included "noise." By "noise" he meant mistakes, as when for example a player intended to cooperate but defectied instead (due, in his model, to mutation) (Lindgren, 1990). He found that neither TFT nor any other pure strategy could be evolutionarily stable. Lindgren's evolving populations developed two particularly interesting phenomena: punctuated equilibria, and evolutionarily stable strategies ${ }^{1}$ (ESS) consisting of long memories and probabilistic or "mixed" strategies. He argued that punctuated equlibria (long periods dominated by particularly successful strategies

\footnotetext{
${ }^{1}$ Lindgren argues that there is in fact no evolutionarily stable pure strategy in the iterated $P D$, but that "in our model the presence of noise implies that every strategy can be regarded as a mixture of two opposite pure strategies, which allows for evolutionary stable strategies to exist" (Lindgren, 1990, 307).
} 
followed by catastrophic collapses) might be necessary in order for dramatically new and better strategies to gain a toehold, and suggested that such punctuated equilibria might be the key to how such complex marvels as eyes evolved; at least in his simulations, these abrupt collapses seemed necessary for the evolution of successful ESS. In contrast to Axelrod, Lindgren's solutions relied entirely on knowledge of past interactions. Axelrod argued that concern for the future is necessary for evolutionary solutions to the PD.

Although these tournaments might shed some light on what is required for rational humans to behave cooperatively, there are difficulties with extending their conclusions to the real world. The solutions they identify rely on pairwise interactions and knowledge of past interactions. These requirements make these solutions questionable for problems such as the TOC, which involves more or less anonymous individuals playing against anonymous multitudes, as well as Nature. Dawes (1980) argues that when iterated PD games are played between two players at a time, as they are in computer tournaments, they are unrepresentative of social dilemmas such as the TOC in general. He gives three reasons: in the real world, "harm for defecting behavior is diffused over a considerable number of players," rather than visited completely on one other player; in the real world, rather than each player knowing with certainty how the other has behaved, defecting behavior may be essentially anonymous; and most important, in pairwise games, "each player has total reinforcement control over the other....Thus, each player can attempt to shape the other's behavior by choice of 
defection and cooperation, while partially determining his or her own outcome by that same choice....In fact, Amnon Rapoport (1967) has shown that if subjects really can influence each others' subsequent choices, then the iterated PD isn't a dilemma at all!" (Dawes, 1980, 183).

Furthermore, TFT may work better than other strategies if players interact pairwise, but if one cannot tell what "TAT" was, or there was a variety of "TATS," then it is impossible to tell what "TIT" should be. TFT thus has no clear meaning when a DM is playing against many others, as well as Nature, simultaneously.

Despite these limitations, it can be argued that the PD, as a limiting case and provocative paradox, has been invaluable to the process of thinking through the causes of, and difficulties in solving, social dilemmas (Rapoport, 1967). Solutions to the commons dilemma require a more complex model, however, because of the reasons outlined above: the real players in the TOC perceive that they are playing different games (e.g., PD, Chicken, some mix of the two, or something else), they may consider multiple, conflicting objectives in their decisions; the probabilistic aspects of their choices are important and cannot be modeled effectively by game theory; and unlike the players in existing computer models, they are not restricted to pairwise interactions and knowledge of past histories for their solutions.

When players are assuming different payoff structures and rules, and those payoffs and rules depend on beliefs and moral values, the greater power and flexibility of decision theory may be required if a solution is to be found. The assumption that all 
players are strictly rational, have a role to play, and are playing the same game is fundamental to game theory. If one of the players is Nature, these assumptions become problematic. For example, although there are many definitions of rationality, in game theory, "rationality" has to do with choosing the course of action that is expected to provide the most of whatever the player desires. By this kind of definition, Nature may or may not be called "rational," but Nature's rationality (if there is suich a thing) is most likely fundamentally different than human players' rationality. Nature/may be playing a very different game than the humans are (or than humans think Nature is playing), and can certainly change the rules without telling anyone. Nature's role in the TOC is as a source of uncertainty, and because decision theory can handle uncertainty so much more easily than can game theory, it is thus a more useful tool.

If all of the players cannot be assumed to be playing the same game, or even having an effective part to play, game theory may be helpful in thinking through the various strategies different kinds of players might select, but it will be of little help in finding a solution. Game theory cannot include the impact of DMs' conflicting probability assessments, and does not explicitly account for the tradeoffs inherent to multiple objectives as easily as does decision theory. In the N-person PD, players' ordinal preferences are all the same, uncertainty plays no role, and potential tradeoffs among multiple attributes are invisible. In the real world, if a player values cooperation because of a belief that cooperation is morally right, and this player believes it is highly probable that the other player will cooperate, then this player will choose the 
"cooperate" option rather than the "defect" option because not only years in prison but also moral precepts are important; the additional attribute changes the payoffs so that the payoffs are no longer consistent with the PD. In sum, decision theory is a more powerful tool than game theory for dealing with uncertainty. Game theory is more useful for dealing with strategic aspects of a situation arising from the potential for cooperation and competition among actors.

The real issue, however, is not the game theory/decision theory dichotomy, but rather whether the TOC is more productively modeled as a game against Nature (i.e., as a multiattribute decision tree), a game against other players (as a game theory model), or even as a game against Nature and other players (i.e., some mix of the decision and game theory approaches). In particular, if the strategic aspects of the problem - the aspects of competition and cooperation among players - are important, then game theory has much to contribute; if the probabilistic aspects of the problem - the uncertainties about the state of the commons and the actions by other players - are important, then decision theory has more to contribute. Both approaches in fact have important contributions to make. 


\section{Chapter IV}

\section{POTENTIALSOLUTIONS}

Given the complexities of human decision-making, and the dilemmas resulting from the conflicts between our biological heritage and our biological future, escaping the TOC is a truly difficult challenge, because as Hardin and Baden so eloquently put it (Hardin and Baden, 1977, xii):

Memory decays; subconscious denial blinds; private interest corrupts; and long-standing institutions seek (so to speak) their own survival. In culture, as in biological ecosystems, we can never do merely one thing. Even when we perceive the errors of the past and the dangers of the future it is not obvious how we should, or can, alter human institutions to improve human welfare. In this crowded world of ours ummanaged commons are no longer tolerable: but how shall we manage them? What core norms of society need to be altered, and how?

It is also important to ask what we really mean by "solution." At a minimum, "solution" could mean merely that $H$. sapiens does not go extinct. The tragedy Hardin feared was defined only as "ruin," which in the context of his essay did not appear to mean extinction, necessarily, but rather the condition where Malthusian feedback 
mechanisms of "misery and vice" hold the population in check. In Malthus' framework, humans may be able to survive, but unless we deliberately control our population growth, we will often be miserable. In the literature about solutions to the TOC, clearcut objectives for a solution are rarely (if ever) defined, but the assumption appears to be that mere survival, though necessary, is not sufficient. Since these objectives appear to be shared by the researchers who have written about solutions to the TOC, in this work it is assumed that a "solution" results not only in survival of the human species, but also the survival of the commons, both at some desirable level of quality.

Many different solutions have been proposed by researchers and philosophers in many fields. Costanza, for example, argues that "All animals capable of choice can be trapped with the right bait. Intelligent ones can learn to avoid traps. Ingenious ones can even escape from traps. If we are to survive, we need to exercise our vaunted intelligence and ingenuity to see, avoid, and escape from the many complex traps we have laid for ourselves" (Costanza, 1987, 412). He cites a list of potential solutions developed by Cross and Guyer, consisting of "education (about the long-term, distributed impacts); insurance; superordinate authority (i.e., legal systems, government, and religion); and converting the trap to a tradeoff" (Costanza, 1987, 409).

Heinen and Low similarly identified five categories of proposed solutions: "information/education programmes, social incentives (through direct reciprocity), economic incentives (through small fines, etc.), the formation of coalitions, and broad 
governmental regulations" (Heinen and Low, 1992, 111). From their survey of empirical studies, they found considerable support for the hypothesis that "solutions will be easier for problems which are smaller in scale, and more immediate to the actor, than for those that are larger and farther away...in general, as problems become larger in scale, more difficult and drastic solutions will be required" (Heinen and Low, 1992, 111). They also found that one of the most important factors in preventing commons tragedies is the presence of rapid and clear feedback (Heinen and Low, 1992; Low and Heinen, 1993). They reasoned that "if enhancement of individual and familial survival and reproduction is the trait favoured by natural selection...resources should be most conservatively used when there is rapid and clear feedback regarding the impact on family and individual welfare" (Heinen and Low, 1992, 108).

They also argue that, unfortunately, given "our evolved psychological mechanisms," the most important problems are also often the most difficult (Heinen and Low, 1992, 109):

The easiest problems are those in which the costs are paid, and the benefits derived, by the same individuals, and the benefits come quickly...; in such cases, simply having the correct information may lead to a solution. When costs or benefits accrue at higher-than-individual levels, there is a potential for conflicts of interest within a level (e.g. gasoline prices: we all agree that something must be done, but we loathe to inflict taxes)....The most difficult cases are those such as global warming: the costs are incurred from the individual to the national levels...and the costs must be paid now for potential benefits in, say, 20 to 50 years. In such cases, anyone asked to pay a cost now is predicted to discount the value of information, the benefits to be derived, etc. In sum, the farther any case is from the individual costs, individual benefits corner, and the longer the time-scale, the more difficult it will be to 
solve.

In this survey of the literature, the proposed solutions appear to fall into six, somewhat overlapping, categories: technology, community identification, financial incentives, property rights, regulatory control, and population control. These approaches rely on changing the rules of the TOC (technology and property rights), changing the payoffs (community identification and financial incentives), or both (regulatory control and population control). The technology, property rights, community identification, and financial incentives solutions are based on an assumption that the commons can be preserved while still allowing more-or-less free individual choice decision-making. In contrast, the regulatory control approach assumes that decision-making should not be left to individuals, but should instead be regulated by an outside body.

Hardin's only solution — population control — is in reality a commons dilemma of its own. If Nature somehow achieved it for us (via, say, a pandemic or reduced fertility), then free choice decision-making could be preserved. If not, then according to Hardin, free choice in reproduction is not acceptable, because "freedom to breed will bring ruin to all" (Hardin, 1968, 29). Coercion, "preferably mutual", is thus required (Hardin, 1968). 


\section{TECHNOLOGY}

Throughout history, new scientific discoveries have repeatedly made "a mockery of old statements of impossibility," as Hardin puts it. As a result, "many nonscientists (but few scientists) think that anything we can dream of we can have (sooner or later)...Maybe tomorrow someone will invent an antigravity machine or find a way to travel faster than the speed of light. Who is to say what is impossible? The 'Who is to say?' of the cornucopists opens the mind's door to all conceivabilities" (Hardin, 1993, 68).

The "cornucopist" argument is that doomsayers have always been with us and have usually been wrong (Hardin, 1993). The reason doomsayers' prophesies fail is that it is impossible for forecasters to anticipate technological advances which, invented often by necessity, inevitably arrive to save the day. There may be limits on nonrenewable resources, but there are no limits on human ingenuity (Simon and Wildavsky, 1993). Groundwater may become increasingly polluted, for example, but technology will find a way to clean it up: all that is required is an increased dedication of technological resources.

As technology improves people's standards of living, people become healthier and better educated, so that they also tend to become more responsible citizens; better ways can then be found to manage commons - or so the argument goes (Dietz and Rosa, 1994; Morowitz, 1991; Pimm, et al, 1995; Simon and Wildavsky, 1993; Wilson, 
1993). The regeneration rate can be improved, say with fertilizers or genetic improvements in crops and livestock. People can recycle more, and recycling can become more efficient, the end products more useful, and so on. In the extreme, the most optimistic cornucopians believe that the infinite substitutability of factors such as labor and capital means that, as Nobel prize-winning economist Robert Solow puts it, "[T]he world can, in effect get along without natural resources" (Daly, 1980, 7).

The pessimists, however, disagree. There is no technical or structural solution to the commons problem over the long run, they say; ultimately, no matter how a commons is managed, its final fate is determined by the number of people exploiting it (Hardin and Baden, 1977). Along these lines, Daily and Ehrlich argue that despite the fact that there is a widespread belief that "technological advances will sufficiently lower per-capita impacts so that no major changes in lifestyle will be necessary," there is an inescapable limit: "technological achievements cannot make biophysical carrying capacity infinite... The bottom line is that the laws of thermodynamics inevitably limit biophysical carrying capacity if shortages of inputs or ecological collapse do not intervene first" (Daily and Ehrlich, 1992, 763).

Some even argue that technology is part of the problem, rather than the catchall solution. Environmental ethicist Georgescu-Roegen is one of the best-known advocates of simpler lifestyles, particularly in the wealthy countries; he argues that too many resources are wasted on trivial wants, whereas for the sake of long term sustainability and higher quality of life, resources should only be used for necessary, 
rather than "nonvital" purposes (Georgescu-Roegen, 1971, 21):

...the maximum of life quantity requires the minimum rate of natural resources depletion. By using these resources too quickly, man throws away that part of solar energy that will still be reaching the earth for a long time after he has departed. And everything that man has done in the last two hundred years or so puts him in the position of a fantastic spendthrift. There can be no doubt about it: any use of natural resources for the satisfaction of nonvital needs means a smaller quantity of life in the future. If we understand well the problem, the best use of our iron resources is to produce plows or harrows as they are needed, not Rolls Royces, not even agricultural tractors.

\section{COMMUNITY IDENTIFICATION AND MORAL CONSIDERATIONS}

Human beings have evolved not only to be selfishly biological competitors, but also to be strongly social, and thus susceptible to social forces such as religion, conscience, pride, and reciprocity. Cooperative tendencies might be built upon to manage common resources more rationally. For example, Heinen and Low suggest that, "Because of our inescapable history as a long-lived, social primate, we possess evolved proximate mechanisms to maximize our functioning in the social context. If we can play upon these evolved mechanisms again, we may be able to promote conservation strategies more effectively than if we require conscious cost-benefit calculations, or rely primarily upon economic costs and benefits" (Heinen and Low, 1992, 112).

Cooperation appears to be more likely when people do not feel threatened by 
scarcity, due either to actual shortages, or to other people's competitive behaviors. For people to not feel threatened by scarcity, the commons needs to be so large with respect to the population that there is little motivation for competitive (or, for that matter, cooperative) behaviors, or people need to feel connected enough to one another that sharing is valued more highly than the potential profit available from competition. Ostrom, for example, found in an empirical survey that common-pool resources could be sustainably managed when "individuals have shared a past and expect to share a future [where] it is important for individuals to maintain their reputations as reliable members of the community" (Ostrom, 1990, 88).

Heinen and Low note that "The most successful coalitions comprise individuals who have convergent interests: e.g. kin...monogamous mates...or frequently reciprocating individuals.... While all of us are loath to inflict costs upon ourselves, there is a possibility that we can use coalitions - which will sometimes accept small costs in order to impose greater costs on others or on society at large - to help us to 'leapfrog' from small-scale successes to larger-scale successes" (Heinen and Low, 1992, 113).

These arguments also appear to be supported by a number of studies of nomadic tribes and fishing villages in Africa (Bassett, 1988; Bencherifa and Johnson, 1991; Monbiot, 1994; Ntiamoa-Baidu, 1991; Stott, 1991). These researchers found that grazing and fishing commonses apparently worked for centuries, as long as their use was mediated by tribal customs and taboos. Unfortunately, when historical 
migration routes have been cut off or traditional beliefs altered by the increasing impact of European and American politics and culture, these commonses have collapsed.

Dawes and others have found empirically that cooperation is much more likely when groups are smaller and more homogeneous, somewhat more likely if choice is public rather than anonymous, and significantly more likely if subjects believe others will cooperate (Dawes, 1980). Rapoport accidentally discovered that even allowing experimental subjects to chat by the water cooler during a break significantly improved the rates of cooperation in N-person iterated PD experiments (Dawes, 1980). Since one of the most important defining features of the $\mathrm{PD}$ is the requirement that one's own choice is independent of what other player(s) may do, then people who change their choices because of others' expected behaviors are not, by definition, participating in a PD.

Some environmental ethicists and "deep" ecologists (Ehrenfeld, 1981; Etzioni, 1988; Sagoff, 1995; White, 1995; Wilson, 1992, 1993) call on human responsibilities to future humans as well as to lother species. Weiss argues for "education to foster a new planetary ethos rooted in a sense of belonging to a community of past, present, and future generations - all of which are responsible for the use and care of planet Earth" (Weiss, 1990, 10). Wilson theorizes that humans have an innate need to interact with, and care for, the natural world (the "biophilia hypothesis," see Wilson, 1984 and 1992). Working to invoke thiis presumably biologically-based need, and educating people about our responsibilities to future humans and other species, is an approach often used 
by biologists and environmental ethicists, perhaps because caring about the biosphere was what drew them to their disciplines in the first place.

People do seem to be sensitive to moral suasion in many contexts. Dawes, for example, found that the tendency of experimental subjects to cooperate in iterated Nperson PD games was significantly improved by "a 938 word sermon about group benefit, exploitation, whales, ethics, and so on" (Dawes, 1980, 188). Costanza supports the view that in some cases, relatively rigid social structures such as religion might work, but he also points out that "...there are problems with religion as a means to avoid social traps. The moral code must be relatively static to allow beliefs learned early in life to remain in force later. And it requires a relatively homogeneous community of like-minded believers for religion to be truly effective" (Costanza, 1987, 410).

Hardin expresses little faith in the efficacy of moral arguments, because "appeals to conscience" place people in double binds. People being preached to may feel that the person making the appeal regards them as "simpletons" (Hardin, 1968). The double bind occurs because if the "swayee" acts consistently with his or her newlyenlightened conscience, it could mean feeling taken advantage of, particularly if the sermonizer may not be trusted to also act conscientiously. The only way this double bind could be avoided, Dawes says, is if both the person being swayed and the person making the appeal feel bound together by a similar set of values: if, as Dawes says, "the person making the appeal also has a regard for his own 'clear conscience'...then he is 
equally bound" (Dawes, 1980, 179).

In effect, proponents of solving the TOC through education are hoping to change social values. It can take a long time to change social values, however - time which some believe we may not have. In addition, when the underlying game is PD or Chicken, it does not take too many defectors to ruin things foi everyone else. Thus, "For education to be effective in avoiding traps involving many individuals, all the participants must be educated" (Costanza, 1987, 409), quite an ambitious undertaking.

The key may be that education has an effect on behavior primarily when it provides information about near-term potential impacts on individuals (and their near kin). Thus, we would expect that as costs of not sharing or cooperating, and/or benefits of sharing or cooperating, become more salient, less-selfish behavior would become more attractive. It may be that if education can make resource scarcities more apparent, the payoffs will change.

Could they change enough? Dawes argues that the answer is "No, for while utilities associated with altruism, norms, and conscience may be made salient by knowledge, they do not necessarily overwhelm those associated with the payoffs. Repugnant as it may be from a normative point of view, moral and monetary (or survival) utilities combine in a compensatory fashion for most people" (Dawes, 1980, 191). That is, "Everyone may not have his or her price, but it does not require a systematic survey to establish that most people in the world will jompromise his or her [sic] altruistic or ethical values for money or survival" (Dawes, 1980, 191). 
Unfortunately, successful community-based commons management examples in the literature are restricted to small, usually primitive, homogeneous communities with low rates of population growth and shared ownership not only of the commons but also of any proceeds from the commons. It may be that community-based commons management methods only work as long as population is small relative to the commons, or at any rate is small enough, and homogeneous enough, that social cohesiveness can provide the necessary control. Under these conditions, there is in fact no dilemma and thus no tragedy. Unfortunately, there are no examples where community-based commons management has been shown to work when the population of the community grew beyond the carrying capacity of the commons.

\section{FINANCIAL INCENTIVES}

Some researchers argue that because $\mid$ we are biologically selfish organisms as well as social ones, solving the TOC may require stronger incentives than social and cultural mechanisms alone. Heinen and Low । (Heinen and Low, 1992, 107), for example, point out that:

If we suggest...that everyone should pay an immediate, relatively small cost...in the interests of gaining long-term global benefits which will be shared with non-relatives and competitors, we are asking for behaviours that have no evolutionary $\mid$ precedent. When we ask people to do things that cost them individually, with no benefit in the short term, and no matter how sensible they may be, we see defection; it hasn't worked as a widespread strategy in the past, and we see no convincing evidence that it will in the future. 
Appealing as a solution to the TOC based on cooperation might be, Daily and Ehrlich offer the view that, "Planning a world for highly cooperative, antimaterialistic, ecologically sensitive vegetarians would be of little value in correcting today's situation...In short, it seems prudent to evaluate the problem of sustainability for selfish, myopic people who are poorly organized politically, socially, and economically" (Daily and Ehrlich, 1992, 763). Hence, economic incentives.

To an economist, the problem with a commons is that the costs of exploitation are not reflected in the net benefits to the exploiter. The supply-and-demand control system envisioned by Adam Smith relies on prices for the linkages, but when supply and demand are decoupled by faulty pricing mechanism, the invisible hand no longer functions. The result is that, as Daily and Ehrlich $(1992,769)$ put it:

Prices relate to both biophysical and social carrying capacities in at least two important ways. First, underpricing of resources encourages unsustainable management. Underpricing often occurs because future generations have no means of making their demands for a resource known...Prices also play an important role in the rates of innovation. High prices constitute incentives for research and development of technologies that are more efficient or that substitute more abundant for scarce resources.

The implication seems to be that, to solve the TOC, one only has to create a payoff structure that accurately reflects the true long-term cost, supply, and demand functions. Then people will do what they should because they want to. Much has been written recently regarding the possibility of this approach to changing the payoffs inherent to the TOC (Heinen and Low, 1992; Low and Heinen, 1993; Ridley, 1993). 
The general idea is as follows (Heinen and Low, 1992, 108, italics in original):

Humans have evolved to use resources in reproductively selfish ways, and thus, if benefits of conservation can be made to outweigh costs for people - for example through a system of economic or other incentives which confer immediate or very short-term benefits on individuals and/or their families and potential reciprocators - then effective conservation strategies are likely to persist and spread. If this is the case, governments and organizations may find it productive to implement policies that create systems of incentives to conserve: the more immediate the benefit, the more successful should be the outcome.

Daily and Ehrlich similarly favor creating mechanisms that act on the market, such as fees, taxes, and insurance in order to "make short-term incentives consistent with long-term sustainability" (Daily and Ehrlich, 1992, 769). Costanza also favors fees or taxes, arguing that "many theorists believe that this method is the most effective in avoiding and escaping from social traps...one could turn the trap into a trade-off by taxing any consumption above the optimum level for resource stability" (Costanza, 1987, 410).

Costanza has more recently advocated treating commons resources, such as rangeland and fisheries (which he calls "natural capital stocks"), in a way that is similar to how capital is treated by investors: "By maintaining natural capital stocks (preferably by using a natural capital depletion tax), we can satisfy both the skeptics (since resources will be conserved for future generations) and the optimists (since this will raise the price of natural capital depletion and more rapidly induce the technical change they predict)" (Costanza and Daly, 1992, 38). Under this system, just as a responsible 
investment analyst would take into account the acceptable risk of a proposed investment, resource managers would be encouraged to take into account the acceptable risk of resource exploitation through a requirement that discount rates reflect the worst case scenarios for the future.

This question of uncertainty about the future, and what levels of risk we should assume on behalf of future generations, is at the heart of environmental conflicts. Hazardous waste management is a good example of a particularly difficult commons issue because there is often a great deal of ignorance and uncertainty (as well as potentially devastating risks) associated with hazardous waste issues. Costanza argues that in order to find an equitable solution to the problem of hazardous waste, "one must charge the producers of hazardous waste for the ultimate long-run environmental and health costs of these wastes, and the charges must be imposed at the time of the waste's production...[But] how can we charge producers of hazardous wastes for the ultimate long-run costs of their waste if we have no idea what (if any) those costs will actually be?" (Costanza, 1987, 410). Costanza suggests that society charge producers the worst-case costs, given the current knowledge. Producers will then be motivated to undertake research as well as to pollute less, he says.

The "catch" inherent in the idea of using financial incentives, however, is that it is difficult to imagine how, exactly, to put it into effect. As Dawes puts it, "The simplicity of this approach is appealing until we ask who will change the payoffs and how. The almost universal answer to the first question is government, and - 
somewhat surprisingly given the cultural background of the writers — the most common answer to the second question is: through coercion" (Dawes, 1980, 174). Particularly in our political system, although people might agree that changing the payoffs is desirable, in an election year few politicians want to be perceived as "tax and spend" types; as a result, "...the immediate individual costs to a politician promoting tax increases are seldom seen as counterbalanced by the long-term societal gain" (Heinen and Low, 1992, 109). Politicians campaign to lower, not to increase, taxes.

\section{PROPERTY RIGHTS}

If the problem is that important resources are mismanaged when held in common ownership, then perhaps the solution is to eliminate the common ownership. Perhaps Smith's invisible hand would work then, given the chance. The fact that people take better care of what is theirs, than what is shared, is well known: "Aristotle, for example, remarked: 'What is common to the greatest number gets the least amount of care. Men pay most attention to what is their own: they care less for what is common...When everyone has his own sphere of interest...the amount of interest will increase, because each man will feel that he is applying himself to what is his own"' (Hardin and Baden, 1977, xi).

The idea of the private property solution is to put a price on everything, and let demand determine the supply. Thus, for example, everyone would receive a certificate 
for a certain amount of clean air, and those who wished to, could sell their certificates - say, to a factory that wants to pollute. The price of pollution then would be paid by the beneficiaries, so that the problem of externalities would be reduced or eliminated. If people prefer to protect a threatened bird rather than to log a forest, let them get together and buy the forest (a la The Nature Conservancy).

One difficulty with converting publicly-owned resources to private property is in figuring out how to distribute a commons equitably (e.g., who should get it? how should it be priced? by what process should it be sold? what about future generations?). There is also the question of what kinds of laws are needed to protect everyone's long term interests. Hardin asks: "What shall we do? We have several options. We might sell them off as private property. We might keep them as public property, but allocate the right to enter them. The allocation might be on the basis of wealth, by the use of an auction system. It might be on the basis of merit, as defined by some agreed-upon standards. it might be by lottery. Or it might be on a first-come, first-served basis, administered to long queues." Unfortunately, he concludes, "These, I think, are all objectionable" (Hardin, 1968, 21). His objection is that either the distribution would not be equitable ("Every new enclosure of the commons involves the infringement of somebody's personal liberty" (Hardin, 1968, 29)), or the system might accelerate liquidation of a commons that is critical to survival.

Privatizing public resources is no guarantee that the resources will be used sustainably, anyway. In a series of laboratory experiments, Cass and Edney found that 
discount rates and fears about the effects of competition tended to cause problems even when property rights are imposed on commonses. In these experiments, subjects were allowed to draw points from a regenerating common pool. Despite the fact that subjects were always informed of the optimal harvesting strategies, subjects consistently overharvested the common resource. When the points were divided into "territories," subjects harvested optimally only when visibility of resource levels was provided, which is very difficult to do for commonses such as fisheries, groundwater, and clean air. If subjects were uncertain about resource levels, they still harvested their own private territories unsustainably (Cass and Edney, 1978). Fife explains such behavior by pointing out that it makes sense to liquidate a resource if the profit can be reinvested elsewhere for a greater return; the driving mechanism is that different people have different discount rates for different resources, and if there is sufficient uncertainty related to slowly harvesting one resource, it is "rational" to harvest it quickly and invest the proceeds in an enterprise subject to less risk (Fife, 1971).

A further difficulty arises because it may be technically difficult if not impossible to impose property rights on a commons when there are problems with restricting access. These issues have been dealt with recently in ocean fisheries, particularly in New Zealand and Alaska, by issuing individual transferable quotas (ITQs) to commercial fishers to "help match the fishing capacity to the stock's size while easing some of the social dislocation caused by such a transition" (Fujita and Hopkins, 1995, B7). The idea is that if people currently dependent on fishing are issued ITQs, which 
they can trade or sell just as if they were private property, then it is easier to manage access and to reduce total fishing efforts: fishers can be encouraged to go out of business by transferring their ITQs to others, including the state or conservation organizations which will not use them to fish (Jeffreys, 1993).

Unfortunately, ITQs have mainly been tried only in fisheries that were already in trouble, and there have been problems with implementation and enforcement (Duncan, 1995; Mathews, 1995). In a survey of ITQ efforts, Mace found that "The net effect is that, even with property rights, the discount rate... of commercial harvesters is almost certainly higher than that of the general public, particularly that of recreationists and conservationists" (Mace, 1993, 30). She argued that ITQs failed to save New Zealand's orange roughy fishery because managers did not understand the biology of the fish until it was too late (Mace, 1993). There have also been problems in New Zealand's ITQ efforts because political pressures have resulted in increased corporate control at the expense of entrepreneurial artisanal fishers (Duncan, 1995). Enforcement has also turned out to be a serious problem (Mace, 1993; Duncan, 1995).

\section{REGULATORY CONTROL}

Along the continuum from purely common to purely private ownership there is also a range of various forms of government. In fact, all the solution approaches described here rely on what Hardin calls "mutual coercion" - i.e., government - to 
some degree, because some kind of governmental system is required to provide the structure within which these solutions occur. However, all the solutions discussed above rely on more-or-less manipulated free choice: technological solutions change the rules so that people can harvest as they please without causing longterm problems, property rights solutions change the rules to eliminate the elements of the problem due to unrestricted access, and community identification or financial incentives change the payoffs so that people harvest sustainably by preference.

In order to not confuse it with other approaches, the "regulatory control" solution here is narrowly defined, and is essentially what Prugh, Costanza, Daly and others call a "command and control" approach (Prugh et al, 1995). For the purposes of this work, regulatory control is defined to include only solutions that turn over the responsibility for deciding who gets to harvest, when, and where, to a governmental body. For example, the state Department of Fish and Wildlife determines catch limits, methods, and timing; the Bureau of Land Management determines how many cattle can be grazed, by whom, on which allotments; the U.S. Forest Service determines how many trees can be logged, where, and how. Licenses or allotments may be auctioned off or sold to the public, and in some cases ITQs may fit in this category better than the "private property" category, depending on how strictly they are regulated. The bottom line, and the characteristic that distinguishes regulatory solutions here, is that the govermmem, not the individual, decides whether or not an individual can Take.

Regulatory control solutions work both by changing the rules and by changing 
the payoffs. They change the rules because they restrict access, as do property rights based solutions. The difference between the solutions that are categorized here as "property rights" and those that are "regulatory control" is that "property rights" solutions assume that individual decision-making will lead to the optimal outcome, and "regulatory control" assumes that it cannot. Regulatory control solutions rely on an authoritarian body that decides who gets to harvest, how, and when, according to some assumptions about what the harvest levels should be. Property rights solutions are based on an assumption that the market (i.e., free choice) will and should determine the optimal harvest levels.

For example, ITQs in fisheries fit the "property rights" solution category if the decisions about buying and selling the ITQs can be freely made by individuals, similarly to the way woodlot owners would buy and sell woodlots; i.e., the ITQ approach is truly a property rights approach if it leads to supply and demand controlling harvest levels. If instead, ITQs are simply thinly veiled licensing schemes like those that have traditionally been used by agencies to control the number of fish caught, then they really belong in the "regulatory control" category.

Regulatory control solutions assume that, left to their own devices, individuals will not achieve an optimal harvest level, and that the decision should not rest in their hands. In the limit, of course, regulatory control solutions also work by changing the payoffs, because a DM always has the option of breaking the law, and paying a penalty or going to jail. 
Many, if not most, commonses are in fact regulated by some administrative system. The rationale behind formation of governing structures is that, if people cannot always be depended on to be virtuous, then at least they can create social or political structures to coerce one another for their mutual benefit. The argument is that since humans have evolved to try to take as much of the pie for themselves and their offspring as possible, some kind of structure has to exist to force them to treat one another equitably. Creating a system of controls should make the future more predictable and thus the present more secure, than relying on unpredictable social and economic dynamics. Hardin advocates "mutual coercion mutually agreed upon" (as a means of managing a commons over the short run, but more importantly as a means of regulating population growth over the long run), but points out that the problem with any governmental system is always that of regulating the regulators themselves, who are subject to political pressures as well as ordinary greed (Hardin, 1968).

Administrative systems are intended not only to maintain social stability, but sometimes also to ensure the future. Future generations have no say in decisions made today that may have consequences for them. Given the human tendency to discount the future, there is little natural motivation to forego using resources today in order to ensure that unknown, unrelated people some time in the future might possibly need them. To some, this is an issue that must be dealt with formally. For example, arguing that "every generation receives a natural and cultural legacy in trust from its ancestors and holds it in trust for its descendants," Weiss proposes that an inter-generational (and 
international) trust fund be established, along with an ombudsman whose job it is to speak for future generations" (Weiss, 1990, 10).

The major objections to coercive systems are that, in the long run, they cost too much, and besides, at least on the scale of states or nations, they simply do not work: if they did, there would be some coercively-managed commons today that could be looked to as an example of sustainable management. The reason they do not work is that people try to get around the rules, and there may even be an increased motivation for many to do so if the system seems unfair (Dawes, 1980). Since people may prefer to try to cheat the system, monitoring and enforcement are required; thus, "Governments can forbid or regulate certain actions that have been deemed socially inappropriate. The problem with this approach is that it must be rigidly monitored and enforced, and the strong short-term incentive for individuals to try to ignore or avoid the regulations remains. A police force and legal system are very expensive to maintain" (Costanza, 1987, 409). Dawes agrees, adding that, "Sometimes, in fact, it is not even possible to avoid a dilemma by reward or coercion, because the costs of rewarding people for cooperating or effectively coercing them to do so exceed the gain the society derives from having everyone cooperate rather than defect" (Dawes, 1980, 175).

An additional problem is that if control and decision-making are turned over to an outside (even if theoretically representative) body, the individuals whose cooperation and ingenuity might be required to solve environmental problems may 
begin to perceive government to be "the problem," and thus changes in government (instead of in citizens' behavior) to be somehow the only potential solution. As Heinen and Low put it, "So long as 'government' is considered as a unified, external force to solve environmental problems, we will not solve anything. It is only too easy to suggest that 'the government' should raise gasoline prices, for example: but...the real problem is how to make it worth while to individuals to act in ways that will cause and support such a governmental response" (Heinen and Low, 1992, 113).

\section{POPULATION CONTROL}

It is well accepted that as long as populations are small with respect to a common resource, usage stays far enough below the carrying capacity that the commons will not be unsustainably exploited (Hardin, 1993; Low and Heinen, 1993; McCay, 1978; Patten and Odum, 1981). As Hardin put it, "The commons, if justifiable at all, is justifiable only under conditions of low-population density. As the human population has increased, the commons has had to be abandoned in one aspect after another" (Hardin, 1968, 28). Population control is the only solution that Hardin says will work over the long run. If population control were somehow magically achieved at a sustainable level, the effect would be to change the rules, because if population were small enough, relative to the commons regeneration rate, it would not matter what people did (Hardin, 1968). Population control can also change the payoffs, however, 
because of its effects on supply and demand.

Problems with commonses have become much more pressing as population has grown. For example (Hardin, 1968, 22):

The pollution problem is a consequence of population. It did not much matter how a lonely American frontiersman disposed of his waste...But as population became denser, the natural chemical and biological recycling processes became overloaded, calling for a redefinition of property rights...Using the commons as a cesspool does not harm the general public under frontier conditions, because there is no public; the same behavior in a metropolis is unbearable.

In this view, population growth is the root cause of misery where population is growing the fastest. Daly argues that "No other social or economic index divides the world so clearly and consistently into 'developed' and underdeveloped' as does fertility" (Daly, 1980, 13). Costanza and Daly worry about the widespread belief, particularly among mainstream economists, that growth is not only necessary, but also infinitely sustainable (Costanza and Daly, 1992).

Others argue that more humans born means more human ingenuity available for solving problems; President George Bush said "ev'ery human being represents hands to work, and not just another mouth to feed" (Cohen, 1995, 343). The most optimistic proponents of population growth, such as Ester Bosrup and Julian Simon, may "[acknowledge] that population growth and economic growth create increased demand for resources. But the resulting perceived or anticipated scarcity is presumed to drive technological progress and with it the search for substitutes and increased efficiency. 
Thus, [according to optimists such as Bosrup and Simon] the net effect of population and economic growth...is neutral or even positive" (Dietz and Rosa, 1994, 281).

Dietz describes another popular belief that, although population growth is a problem, it is not the problem; to some, he says, poor management, inefficient use of resources, and careless lifestyles (particularly in rich countries) are the problem (Dietz and Rosa, 1994). Mark Sagoff, for example, argues that problems such as pollution are not due to population but to lifestyles (Sagoff, 1995). The effects of population growth can thus be managed simply by reducing consumption and improving efficiency.

Hardin does not agree, of course. His argument is that ultimately, no technological, economic, or social solution will work over the long run unless population is controlled, and that even if his pejorist perspective is not widely accepted, it is still important to ask, "Whom shall we believe: The Technological Optimists, or the Limits Lobby? If we are wrong, which way of being wrong is more dangerous? What is the proper policy for the true conservatives?" (Hardin, 1971, 15). If we choose the cautious path and decide that population growth must be stopped, the problem then is how to control population, and how to buy the time necessary to do so. Framed this way, all of the above proposals may then be applied to population control, which is a TOC problem in its own right (Cohen, 1995). Absent some devastating pandemic or nuclear holocaust, slowing, stopping, and even reversing population growth would require many generations. It is unlikely that any one approach to preserving important commonses would be adequate, given the current approximately 40 -year doubling time 
for human population, coupled with the increasing per-capita rate of consumption of natural resources around the world. 


\section{Chapter $\mathrm{V}$}

\section{S I G N I F I CA N CE OF WORK}

Biologists, economists, psychologists, and other researchers interested in public policy agree that the root cause of social dilemmas such as the TOC is the fact that when immediate individual interests appear to corflict with long term social and ecological interests, our biological heritage dictates that the immediate individual interests win out. They do not agree on how best to go about solving this problem, nor do they agree on what the solution should look like.

Researchers from different fields frame and define the problem differently. As discussed in Chapter II, biologists and ecologists have traditionally focused on linear models of equilibrium processes in biotic systems, considering human behavior to be little more than a disturbiance input. Social scientists have focused on human decisionmaking and public policy development, considering the biosphere to be little more than a source or inputs and a sink for wastes. Because the problem is neither wholly biological nor wholly social, and the two systems are inextricable linked, both perspectives are needed. 
In the best tradition of systems science, this work provides a powerful simulation methodology which relies on well developed tools from both arenas: EMAV, provided by the field of decision theory, is used to model the social and economic human components; and a stochastic model of a simple natural system, from the field of ecology, is used to model the resource. The feedback loops between the two are modeled via a genetic algorithm, a tool that developed out of the confluence of evolutionary theory and optimization theory.

The goal of this study is to increase our understanding of how the evolution of individual decision processes in a social, political, and economic human system dependent on a slowly regenerating, commonly held natural resource, might impact efforts to solve the TOC. The study contributes to the knowledge base at the levels of both theory and practice in four ways:

1. It develops and demonstrates the usefulness of a powerful new computer modeling methodology that can help policy makers and researchers think about and solve this important and complex problem.

2. It contributes to ecological modeling theory and practice by providing a new way to use a computer to investigate the links between DMs and the natural systems they impact, as well as contributing to evolutionary modeling practice by demonstrating a new way to use the GA.

3. It contributes to decision theory by helping shed light on the ways dynamic interactions of individuals result in the development of, and potential solutions 
to, social dilemmas.

4. It contributes to decision practice by developing and demonstrating a powerful new way to incorporate dynamic processes of feedback and interaction to previously static decision analysis methods and optimization.

\section{A NEW COMPUTER MODELING METHODOLOGY}

The single most important tool for enhancing human problem solving is probably the digital computer, yet there is currently no satisfactory way to simulate social dilemmas using computers. Computer models help people think about complex problems like the TOC because they can be used to explore the effects and interactions of many variables, whereas humans are limited to thinking of at most a few variables at a time. A computer model can help facilitate communication, generate new solutions, and define and bound the problem. A good computer model helps researchers to determine what factors a problem solution is sensitive to, thus making experimental efforts more efficient. Whereas it is very difficult for humans to predict system behaviors when there are nonlinearities and variables that interact, computer simulations do not necessarily have to make predictions, but can allow previously unforeseen behaviors to emerge.

A computer simulation can help people deal with uncertainty. Using a computer simulation to explore interactions between socio-economic and natural processes is 
new and potentially very powerful. Public policy decision-making is full of uncertainties for which little reliable information is available. Experiments can sometimes be conducted on a small scale to try to understand the potential outcomes of a particular policy, but because political processes operate over time scales that are less than decades, while ecosystems operate over time scales that are more like centuries, the results of such experiments - even when they are measurable, which they often are not — are of limited use. Problems that lend themselves so poorly to empirical investigations are often explored by computer simulations.

Paul Edwards (1995) argues that even when models of complex systems are (of necessity) based on gross over-simplifications, they can still be powerful heuristic guides to understanding complex phenomena. Because complex models often must rely on many questionable assumptions and estimates, they can be criticized on the grounds that their estimates are predictively useless at statistically significant levels, he says; but they can be profoundly important to the development and comparison of policy scenarios and forecasting of trends. He cites the "limits to growth" (LTG) models from the 1970s as an example: An inter-disciplinary team of scientists developed a very complex computer model of world-wide resource consumption, population growth, pollution, capital, and agriculture. They concluded that exponential growth rates in consumption and population growth were not sustainable on a finite planet, and sounded an alarm that got a great deal of attention over the next couple of decades. The LTG models were attacked from every quarter for their lack of precision and 
accuracy, and because there was no real empirical validation. The models nonetheless had an important impact on the growing debate about the potential limits to human population growth (Edwards, 1995). The impact was due primarily to the trends they identified and the relative importance of the parameters and variables they used, population growth in particular.

The computer simulation developed by this study shares the LTG models' problem of the lack of good data on which to base empirical validation. The validity of the model has to be assessed on the basis of reasonableness of assumptions about trends and functional interactions of its parameters, rather than on whether the particular parameter values used are consistent with empirical data. The reason for this is that there simply are no empirical data that relate social and ethical values such as those reflected in the EMAV decision models, to the biological parameters of a commons, in the real world - not because this is not an extremely important question, but because it is such a difficult one.

This model uses two primary tools to deal with this lack of data. First is the reliance on very simple and widely accepted sub-models of a commons and of decisionmaking. Second is the reliance, where necessary, on a "best case" approach. For example, humans are not widely believed to make decisions using EMAV, but EMAV is the prescriptive method recommended by economists and cognitive psychologists. Natural resources are subject to many disturbances and generally do not regenerate so predictably nor successfully as the simple model $\mu$ sed here implies. In this approach, the 
question investigated is whether, given the simplest, optimistic assumptions about human decision-making and commons regeneration, it is possible to prevent the commons being destroyed, without controlling population growth. The results are presented as general relationships among model parameters, and between parameters and results, and not as statistically significant numbers which could be compared to empirical data, if any were available.

There is nothing new about any of the methodology of the components of the model; what is new is the way existing methodologies from diverse fields are used together, in order to increase understanding of an important and difficult problem.

\section{CONTRIBUTION TO ECOLOGICAL THEORY AND PRACTICE}

Ecosystem modeling has progressed to the point where landscape-wide evolutionary and successional processes are beginning to be simulated with extraordinary realism. However, despite the fact that human impacts are widely acknowledged to play an extremely important role in natural systems, no one has found a way yet to effectively incorporate human decision processes into dynamic models of natural systems. Although the ecosystem modeled in this study is a very simple one, it is solidly grounded in the field of ecology. Most important, it provides an innovative way to include evolutionary human decision processes in the study of natural biotic systems. 
A number of researchers have called for integrating decision theory into ecological models. Among the first were Fiering and Holling, who in 1974 proposed that decision theoretical tools such as pareto analysis and sensitivity analysis should be applied to dynamic, nonlinear models of system resilience in order to find optimal system management solutions (Fiering and Holling, 1974). Holling and Clark followed up by calling for a "new science of ecological engineering," focusing on the integration of ecology and economic modeling, policy analysis, and decision theory (Holling and Clark, 1975, 250). Bassett later concluded that his field study of grazing on public lands in Africa illustrated the urgent need for more research into the interrelationships between local, individual decision processes, and national or international political processes (Bassett, 1988). Belyaev and Khudoshina recently argued that there is an urgent need for simulation tools that can be used by resource managers to explore the interaction between the biosphere and human resource managers in order to develop ecologically sound guidelines for economic development (Belyaev and Khudoshina, 1992).

Scientists often express frustration with the fact that public policy tends to be driven by the short-term forces driving politicians, whereas the biosphere is driven by the long-term forces driving nature (Edney and Harper, 1978; Holling, 1969; Low and Heinen, 1993). The use of discount rates by economists and resource managers to deal with future outcomes is particularly repugnant to ecologists, because explicit discounting of future outcomes encourages what many see as an ecologically 
destructive human tendency, and because discount rates cause outcomes more than a decade or two in the future to become irrelevant. Because this model relies on an evolutionary model of decision-making and its ultimate impacts, it provides an innovative way to examine how the short-term, political, socio-economic realities of decision processes can ultimately impact the long term constraints of nature - and vice versa - without having to rely on discount rates.

\section{CONTRIBUTION TO DECISION THEORY}

Researchers interested in how people make decisions about social dilemmas have had to rely almost entirely on empirical work, because there are no computer models available that simulate these dilemmas adequately. The only computer models that have been developed to explore social dilemmas have been based on the iterated, $\mathrm{N}$-person PD. Although these models provide interesting insights into cooperation and competition in pairwise interactions between players with known histories, the TOC is more complex than can be represented by these simple models. Perhaps most important, solutions that have been proposed to the TOC in the literature cannot be readily modeled with the $\mathrm{PD}$ because these solutions involve decision attributes other than pure self interest, and take uncertain futures into account, whereas in the PD, neither uncertainty nor non-selfish interest is taken into account.

This work employs the more powerful tool of decision theory to simulate the 
TOC by including characteristics that simpler game theory models cannot include. In particular, the decision model allows explicit DM assessment of multiple objectives and uncertainties. Because the commons in this model changes in response to what the DMs do, and the GA portion of the model simulates the way people's beliefs and values might evolve in response to the dynamics of the commons, a more realistic simulation is provided of the dynamics of commons decision-making than previous PD-based models have been able to do. Psychologists can use this approach to explore potential interactions among decision variables such as DM beliefs (probability estimates), social values (attribute weights), and payoff structures (value functions). By using computer simulations to conduct such sensitivity analyses, researchers can identify variables that might be important to explore empirically. This information can then be used to design more sophisticated experiments to increase understanding of how people make decisions about commonly held resources, and how they respond to the outcomes.

Despite the fact that there has been an enormous amount of research in this area, it is well known that a significant number of people (around a third in most experiments (Dawes, 1980)) do not choose the dominant strategy of defecting in PD experiments, despite the fact that the definition of "rationality" assumed in game theory requires they should. It is hard to believe that thousands of experimental subjects who consistently choose the more socially responsible (and in fact, potentially more jointly beneficial) course of action are actually not "rational". We might conclude instead either that these cooperators are considering attributes (such as morality or social 
identification) other than the tangible payoffs (usually cash or grade points) offered in these experiments, or that they have different beliefs about the utility of the outcomes than defectors do. Although the prescriptive, static approach of modeling decisionmaking with EMAV has proven to be very useful for helping people make better decisions, there are no descriptive computer simulations available that simulate how they really do make decisions. There simply is no computer model of decision-making that will produce rational cooperators in social dilemma games, but it seems quite reasonable to expect that a model such as this one, which includes DMs' beliefs and values, could.

\section{CONTRIBUTION TO DECISION PRACTICE}

This study contributes to the field of decision practice by extending the welldeveloped, static, prescriptive model of decision-making, EMAV, to a more realistic and useful dynamic model of decision-making that incorporates the effects of outcome feedback processes. Cybernetic processes such as feedback and control are playing increasingly important roles in ecological as well as economic modeling (Jeffers, 1988), but the capacity of current decision models to incorporate these processes is primitive. Prescriptive decision practice as it exists today is typically a static process that begins with a decision-maker evaluating the state of the world and estimating the likely outcome of uncertain events, and ends with the decision-maker's selection of a course 
of action. DMs may improve their decision-making abilities by evaluating the success of their past efforts (Russo and Shoemaker, 1989), but the models of the world that DMs have to rely on when they evaluate complex decisions are essentially static.

This is not to say that EMAV does not or cannot take change into account, because incorporation of uncertainty into a decision is one of the main benefits of using EMAV; but the only way EMAV can take uncertainty into account is by assigning probability estimates to uncertain events and calculating the expected value of the outcomes. Decision trees do allow for decision nodes to follow event nodes and, thus, for decisions to be contingent on feedback and external control to some extent. A crude model of a feedback loop could be created in a standard decision tree with a succession of decision nodes and event nodes, but it would necessarily be very ungainly because the complexity would increase geometrically with the number of iterations modeled on a single decision tree. Modeling even a simple choice this way would quickly become daunting, and this is most likely a major reason why it is not ordinarily done. By providing a straightforward way to model the ongoing interactions - the feedback loops - between a DM's choices and the larger system's responses to those choices, this work provides a very powerful tool for evaluating complex and important decisions where feedback may play an important role.

In its prescriptive mode, decision-making is an optimization process, and it is important to note that GAs are already being used for optimization efforts, particularly for nonlinear problems (Axelrod, 1987; Ball and Gimblett, 1992; Riolo, 1992). 
Although Hardin has posited the TOC as a potentially solvable optimization problem with multiple, conflicting objectives (Hardin, 1968), he acknowledges that actually solving it is probably impossible unless society can agree on priorities and objectives. Unfortunately, the TOC, like other social dilemmas, occurs because of conflicting objectives on several levels: within-individual, between individuals, between generations, and between humans and other species. Because of the hierarchically complex relationships among these conflicting objectives, it is difficult to argue that a global optimization function could even be defined.

The GA is a good tool to apply to this kind of problem because its contribution is not typically to find the optimal point in a solution space, but rather to identify a relatively better region. If the solution space itself is changing, as it is in this work, then rather than serving as a global optimization tool, the GA serves as an evolutionary simulation tool. It does this by crudely simulating the way evolution acts as a shortsighted local, rather than a long-term global, optimizing tool itself.

\section{THE TRAGEDY OF MISSING THE POINT}

Hardin's paper, "The Tragedy of the Commons," has been cited over 570 times since it was published in 1968 (Trout, 1995). Many, if not most, of the citations, refer to his discussion of the difficulties in managing resources that are held in common, and they ignore his main point, which was that the difficulties arise because of population 
growth. It is interesting how reluctant people are to grasp his main point, which he was at some pains to make clear (Hardin, 1968, 17):

The class of "no technical solution problems" has members. My thesis is that the "population problem," as conventionally conceived, is a member of this class...It is fair to say that most people who anguish over the population problem are trying to find a way to avoid the evils of overpopulation without relinquishing any of the privileges they now enjoy.

Yet his essay is often criticized (or praised) by authors who make no mention of Hardin's principal thesis, but instead attack (or praise) him for allegedly claiming that the tragedy occurs because of common ownership. A typical example is the argument that the collapse of ocean fisheries is due not to population pressure, but to common ownership: "The current approach to ocean issues has been characterized as a 'tragedy of the commons' (Hardin 1968). The best way to end the tragedy is to end the 'commons'" (Jeffreys, 1993, 28).

Hardin did argue that externalities, and lack of assignable property rights, explain why people may overharvest commonses; but he emphasized that this is not a problem unless population grows beyond the commons carrying capacity. Hardin did not advocate private ownership as a longterm solution, and he did not advocate commons ownership either: "Perhaps the simplest summary of this analysis of man's population problems is this: the commons, if justifiable at all, is only justifiable under conditions of low population density" (Hardin, 1968, 28). The solution he advocated was limiting population growth, through mutual coercion, mutually agreed on. This 
point is often completely overlooked; Hardin would probably say it is overlooked because of psychological denial: population, he says, is a "taboo" issue.

Ecology magazine devoted an entire double issue (March/April, May/June, 1995) to the problem of overfishing. Included were some lengthy attacks on Hardin's "fallacy" of assuming that communal commons management could not work and that property rights solutions would (Duncan, 1995; Editors, 1995; Fairlie, Hagler, and O'Riordan, 1995). Almost nothing was said in the entire issue about the possibility of population growth being the root cause of the problem, nor that population growth might interfere with the proposed solutions. Overfishing was held to be a problem of distributional equity and overcapitalization. The primary solution advanced was a return to simpler technologies and more traditional management based on community identification. None of the articles in this double issue mentioned the fact that Hardin did not dispute the effectiveness of community-based commons management, as long as population is small relative to the commons regeneration capacity; he said that only after population grows beyond the commons carrying capacity do problems inevitably arise (Hardin, 1968).

Berkes et al. launched a similar attack on Hardin. They described several examples where community identification in traditional villages resulted in sustainable commons management for centuries, and where commonses were destroyed only after traditional management was displaced by privatization and/or government control (Berkes, Feeny, McCay, and Acheson, 1989). Again, not a word was said about the 
fact that in their examples, sustainable management only worked under conditions of low population density, and that changes in management occurred simultaneously to, or after, population grew beyond the commons carrying capacity.

In an opinion piece in Scientific American, Monbiot (1994) scathingly attacked Hardin's alleged advocacy of privatizing commonses. Monbiotdescribed the way a grazing commons in Kenya was destroyed after the commons was privatized, again ignoring the role of population growth completely. Responding to Monbiot in a letter to the editor, Hardin argued that the tragedy occurred because the herdsmen exceeded the commons carrying capacity, not because the commons was privatized: Monbiot, he said, had it "180 degrees the wrong way" (Hardin, 1994, 10).

The point here is that any metaphorical model, even one as elegant as Hardin's, is in danger of being misunderstood or misapplied, through ignorance, psychological denial, or deliberate intent. Because this research is also metaphorical in a way that is similar to Hardin's model, its thesis and its applications could also be misunderstood or misapplied. The research question, "Is Hardin right?" is inextricably linked to its logical extension: "Right about what?" In the preceding section, for example, methods for managing commonses were listed and described at some length, but this work is not about how best to manage commonses, any more than Hardin's essay was. The thesis of this work is that Hardin was right in arguing that no social, technological, or economic solution to natural resource destruction can work over the long rum unless population growth is controlled. 
What this means is that the conclusions of this work only apply to the question of whether solutions that can be made to work in the best of all possible worlds (i.e., a computer simulation of rational decision-making, with a predictable commons, and controlled population size), can also be made to work when population is allowed to grow. The results say little about the pragmatic aspects of any of the controlledpopulation solutions, particularly since the sub-models are based on "best case," simplistic, idealized assumptions. The question being investigated is how population growth could impact solutions that might work, given a set of idealized assumptions. Hardin said that the way population growth will impact any solution is that it will eventually make it fail: "Freedom to breed will bring ruin to all" (Hardin, 1968, 29). That is also the thesis of this work.

This concludes the background and problem formulation portions of this dissertation. There are two key requirements of a simulation of the TOC: (1) it must simulate the way individually "rational" decision processes of resource managers lead to the collectively irrational destruction of a commonly-held resource, and, more importantly, (2) it must be capable of simulating the way those decision processes might have to change in order to avoid the tragedy. Previous computer simulations based on the PD do not meet these requirements because they assume the players interact pairwise and know the histories of their interactions, and they do not take into account the multiple objectives and considerations of uncertainty as real DMs would do. The purpose of this work is to develop a computer simulation that addresses the 
limitations of previous computer simulations, in order to explore the impacts of population growth on existing proposals for solving the TOC, and to provide new insights into this important class of problems.

The remaining sections of this dissertation describe the methodology and findings of the research. In Chapter VI, the methodology of the integrated model is described in detail. In Chapter VII, the model development and some results are described. In Chapter VIII, the solutions are simulated and discussed. Chapter IX is a summary and conclusion. 


\section{Chapter VI}

\section{MODELING METHODOLOGY}

This simulation models the interactions among the DMs managing a commonlyheld resource and the response of the resource to their actions. The decision processes of resource managers are modeled using EMAV from the field of decision theory (Keeney and Raiffa, 1976; von Winterfeldt and Edwards, 1986). The commons is modeled using a simple ecological model such as those described by Odum (1969). The simulated evolution of decision processes, and the feedback linkage between the DMs and the commons, are modeled with artificial life simulation methodology based on Holland's (1975) work with GAs.

This chapter begins with a broad overview of the modeling methodology. In the following sections of the chapter, the commons model, GA, decision model, and fitness evaluation are described in more detail. 


\section{MODEL OVERVIEW}

In this model, different types of DMs compete with one another through many iterations for shares of a regenerating common resource subject to stochastic natural disturbances. Hardin's commons was a pasture grazed by cattle, but in this research, the commons is a population of fish in a lake, which is fished by the people in a nearby village. A fishery commons was chosen instead of a grazing commons for three reasons: ease of modeling, personal interest, and impontance of the problem.

Well-developed simple population models of fish are readily available, whereas it was difficult to know where to begin to model a cattle-and-rangeland commons system. Although some cattle are grazed on public lands in the developed world, most such "commonses" are so tightly regulated that they no longer resemble the kind of commons Hardin was discussing. Ocean fisheries, on the other hand, are almost unavoidably commonses on a world-wide basis, because fish move around too much for any one country (let alone any one fisher) to maintain control over stocks its citizens might consider "theirs." In addition, over half the fish eaten today comes from inshore marine commonses locally managed as more or less common-access artisanal (primitive or subsistence) fisheries (Fairlie, et al., 1995). These fisheries typically provide one-third to one-half of the protein in the parts of the world where population is growing the fastest. The world's fisheries are in an extreme crisis condition: the UN Food and Agriculture Organization rates $70 \%$ of the world's fish stocks as "depleted" 
or "almost depleted," and total catches have been dropping precipitously all over the world (e.g., by a third in the northwest Atlantic over the last 20 years) (Editors, 1995). The conceptual model is thus an artisanal fishery: a village and a lake. The DMs are the potential fishers in the village, and the commons is the stock of fish in the lake.

A flowchart of the four interacting model components (the commons model, the village model, the decision model, and the GA) is shown in Figure 6.1. At the beginning of each iteration $i$, the state of the commons is $x_{i}$. The initial population of villagers $P o p_{i}$ consists of a mix of different kinds of decision models. During each iteration of time increment $\Delta t$, the population grows and changes from $P o p_{i}$ to $P o p_{t+1}$, and the state of the commons changes from $x_{i}$ to $x_{i+1}$.

In the "Decision Models" part of Figure 6.1, the DM genotypes determine how each DM will evaluate the current state of the commons and the likely actions by others, in order to set up their decision trees. The DMs use those decision trees to make choices. A DM who decides to take a unit of the commons is a Taker, and a DM who decides not to is a Refrainer. The payoffs received by individual DMs are determined by their own choices and their genotypes (their genotypes represent their expectations and values), as well as by how many DMs decide to Take, and the new state of the commons $x_{i+1}$.

In the "Commons Model" part of Figure 6.1, $x$ is decreased by the number of Takers, and increased by its regeneration rate, for a net change from the old state $x_{i}$ to the new state of the commons $x_{i+1}$. 


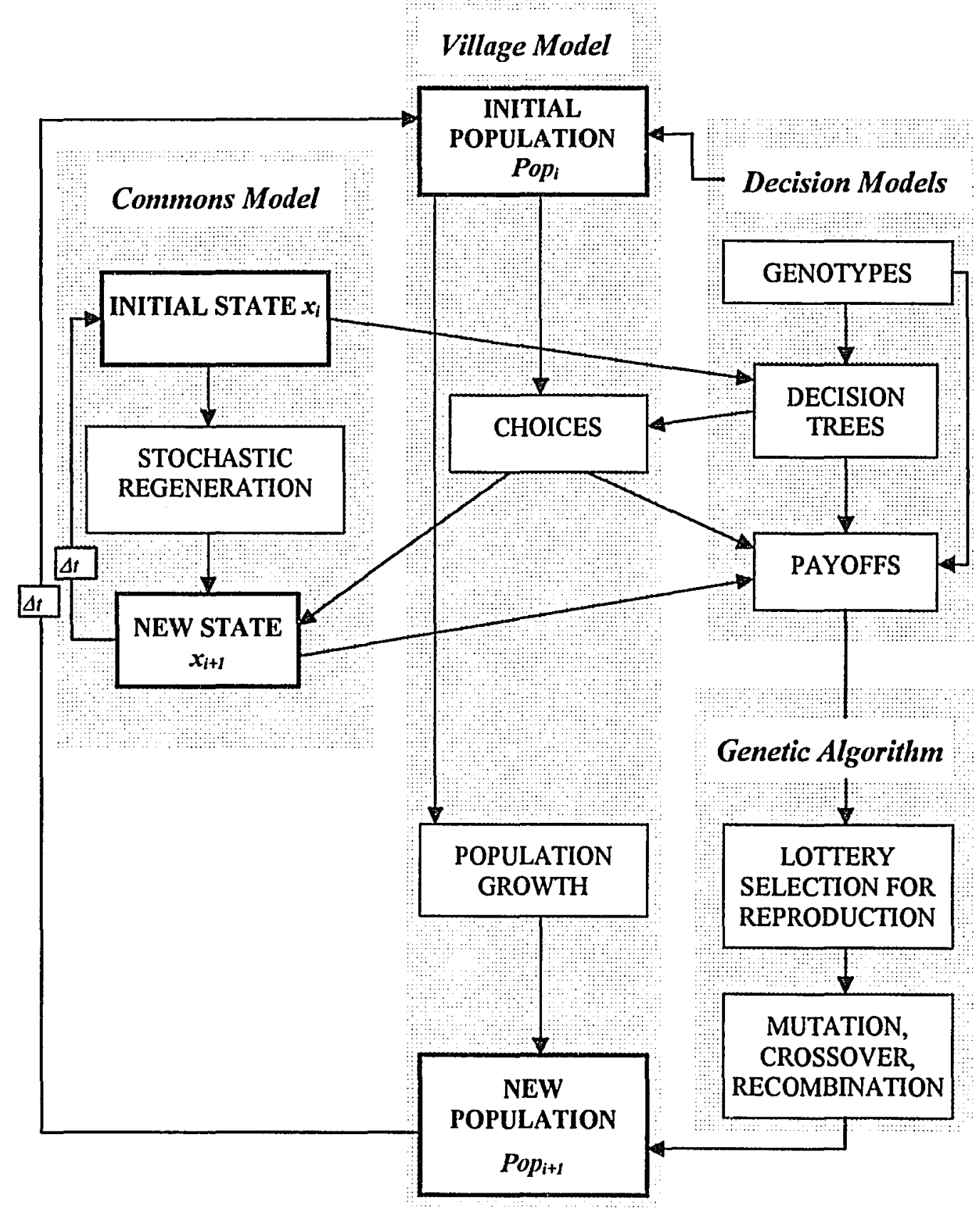

Figure 6.1. Flowchart of the four interacting model components of the TOC simulation. 
In the "GA Model" part of Figure 6.1, the relative magnitudes of the DM payoffs are used in a simulated lottery to select the genotypes that are used to create the population for the next iteration. The selected genotypes are subjected to mutation and recombination to produce the new population Pop $_{l+l}$, and the cycle starts all over again with the next iteration. Because of the probabilistic selection process of the GA, beliefs and values of DMs that provide relatively higher payoffs in one iteration will tend to be relatively more prevalent in the next. However, the commons is changing too, as is the composition of the population, so that DMs who are more successful when the commons is in, say, excellent condition, may not do so well after the commons becomes degraded.

The feedback loops between the DMs and the commons could be stabilizing or destabilizing, depending on the particular set of conditions and simulation parameters. For example, if the number of units of commons harvested is greater than the commons regeneration rate, then the state of the commons $x$ will decrease. As $x$ becomes increasingly scarce, there is less for the Takers to divide among themselves, but scarcity can increase the price they can get. Pessimists may leave the market but optimists may join it. As $x$ declines, some DMs who Refrain for moral reasons may actually get more benefit from a lower level of $x$ because they feel more virtuous about not Taking from a threatened commons than they would about not Taking from an abundant commons. If potential Takers leave the market (i.e., become Refrainers) then $x$ can again increase, eventually attracting more Takers back into the market, and the cycle 
repeats. This feedback loop does not necessarily lead to stability in this model nor in the real world: it could lead instead to an unstable cycle or an irreversible collapse of the commons, depending on the relationship between the rate of change of the numbers of Takers, the time lags, and the stochastic regeneration rate of the commons.

For example, if $x$ falls below the level at which the commons can regenerate itself (its Minimum Viable Level $M V L$ ), the change is irreversible, so even if there are no Takers, it cannot recover. On the other hand, if the regeneration rate of the commons is faster than the growth rate of Takers, there is little to check extraction growth, at least for awhile. As the regeneration rate and/or the extraction growth rate changes, then at any one iteration it is impossible to say which way the net feedback mechanism will be working. Whether the effect of these interactions is ultimately to accelerate or retard any changes depends on how tightly coupled the DM model and the commons model are, and the relative magnitude and sign of changes in the commons and the DM population composition over any series of iterations.

Simulated evolution here is not simulated biological evolution, but it is rather a mathematical tool used for modeling social change. What "evolves" in this model is the values and beliefs of the population that uses the commons, not the genetic material carried in the cells of human beings. The only biological elements simulated in this model are the commons regeneration and the increase in numbers of the human population. Population growth is simulated as a simple exponential growth function, driven by unidentified factors that have nothing to do with the GA nor with this model. 
The GA simulates changes in beliefs and values inside the heads of real people as they observe the results of their actions and those of other people. The distribution of genotypes in each new population of DMs at the end of each iteration is not a distribution of actual progeny of living decision-making people, but a distribution of beliefs and values held by living decision-making people (see the "Fitness" section later in this chapter).

The interacting sub-models of Figure 6.1 represent a simplistic picture of the interaction between human decision making and a natural resource. Garrett Hardin's formulation of the TOC was even simpler, however. This research is based on an argument that his metaphorical model was an inadequate representation of reality, and that the proposed model is better. It is not being claimed here that the proposed model is an adequate representation of reality, but only that it is an improvement over Hardin's. The direction of improvement is defined to be the direction of model complexity that helps increase understanding of how this difficult problem may be solved.

In the real world, the commons dilemma is so complex that it is difficult, if not impossible, to think about unless it is somehow simplified. The great advantage of very simple metaphors like Hardin's TOC is that they are easy to understand and intuitively appealing. Their simplicity lends them a logical neatness that makes the resulting conclusions highly compelling. The danger is that their very simplicity, intuitive appeal, and compelling conclusions may in some important cases not be faithful enough to the 
real world for the conclusions to be valid.

If the simple model is suspected of being not quite faithful enough to the real world to solve an important problem, then a more complex model may be required. Unfortunately, as a model becomes more complex it may be even more difficult to assess its validity because the difficulty in thinking about the model increases as the model complexity increlases. Just because the world is more complex than a simple model does not mean that a more complex model is necessarily any more valid than the simple one.

One way to go about model development is to start with a very simple model (such as the TOC) that is believed to be realistic under some simple set of circumstances, and add one or two new aspects (such as probability estimates, consideration of conscience, and feedback loops) that are thought to be potentillly important. If these new elements change the outcomes under the simple set of circumstances, and are arguably important in the real world, then further investigation of the importance and impacts of these new elements might be justified. The results would not necessarily mean that the new, more complex model is making correct predictions; they would only indicate that something important that was left out has now been added, and that additional important aspects of the problem are being considered. More lines of inquiry may then be opened, and progress towards a more accurate predictor model, and greater depth and breadth of understanding can $^{\text {be }}$ made. If the new elements do not change the outcomes from the simpler model, then 
this finding should help validate the simpler model; it should not, however, necessarily halt the search for more helpful models when the conclusions produced by the simpler models are so pessimistic.

The remaining four sections of this chapter provide more detailed discussions about how the commons is modeled, how the GA works, how the DMs make their decisions, and how fitness is assessed. 


\section{THE COMMONS}

A simple generic model of a commons is used. The state of the commons is expressed as the number of units $x$ available. The nominal regeneration function $r(x)$ is based on a logistic population model, where the rate of change of $x$ depends on the current state $x$ (Grant, 1986, 248):

(6.1) $r(x)=\frac{d x(x)}{d t}=r_{0} x-r_{0} x^{2} / K$

where $x$ is the state of the commons, $r_{0}$ is the regeneration rate constant, $K$ is the maximum value $x$ can be, and $r(x)$ is the rate of change of the commons $d x / d t$, at a particular commons state $x$ (see Figure 6.2).

The discrete version of Eq. (6.1) used in the simulation is:

(6.2) $r\left(x_{i}\right)=r_{0} x_{i}-r_{0} x_{i}^{2} / K$

where $r\left(x_{i}\right)=x_{i+1}-x_{i}$.

The state of the commons is determined not only by its nominal regeneration function $r(x)$, but also by the DMs' actions and Nature's stochasticity. Stochastic regeneration is represented as a normal distribution $N$ with mean $r(x)$ and a standard deviation that is proportional to the mean. The parameter Stoch determines the proportionality constant (i.e., Stoch is the coefficient of variation, or the standard deviation normalized by the mean). 


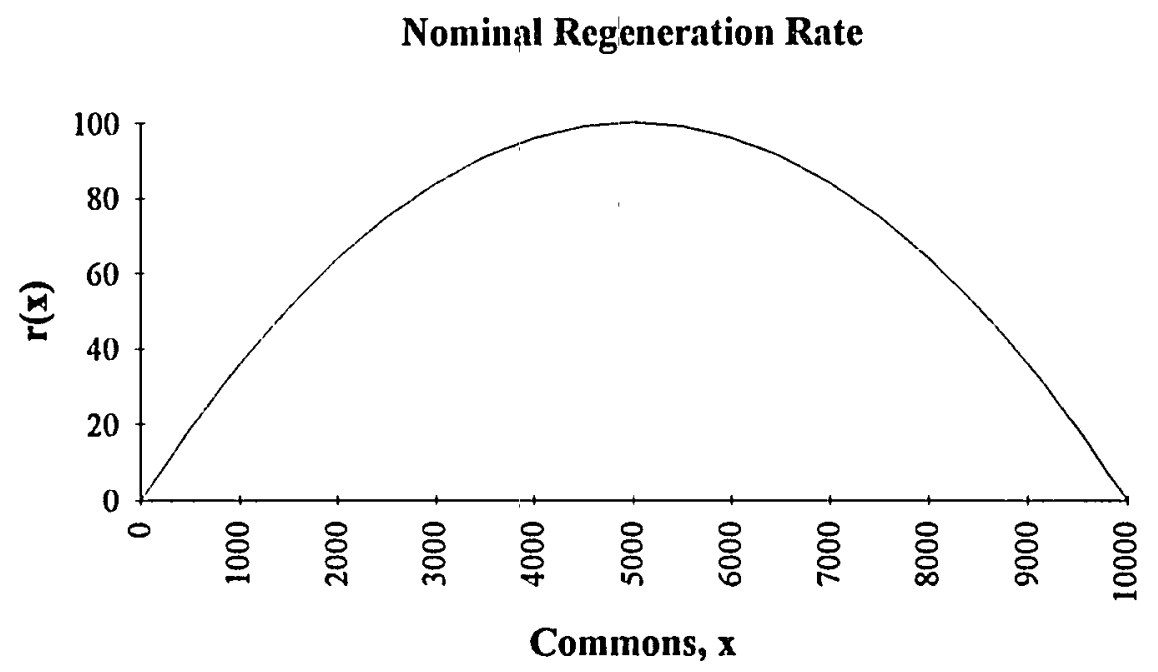

Figure 6.2. Nominal commons regeneration rate curve, for $r_{0}=0.04$.

The change in state of the commons during any iteration (from "initial state" $x_{i}$ to "new state" $x_{i+1}$ in Figure 6.1) is determined by the initial state of the commons $x_{i}$, by actions of the current population of DMs (i.e., the number of Takers $T_{i}$ ), stochasticity Stoch, and the commons regeneration rate $r\left(x_{\nu}\right)$, or:

(6.3) $x_{i+1}=x_{i}-T_{i}+N\left\{r\left(x_{i}\right)\right.$, Stoch *r( $\left.\left.x_{i}^{\prime}\right)\right\}$

where $N\left\{r\left(x_{i}\right)\right.$, Stoch $\left.* r\left(x_{i}\right)\right\}$ is a normal distribution with mean $r\left(x_{i}\right)$ and standard deviation Stoch * $r\left(x_{i}\right)$. 


\section{THE GENETIC ALGORITHM}

GAs work by simulating evolutionary adaptation. In the computer code, each individual in an evolving population is represented by a string (the "genotype") of binary variables (the "genes"), each of which assumes a value from the set of possible values ("alleles").

GAs were developed by researchers interested in simulating life processes on computers (Holland, 1992). In these biological models, genotypes represent individuals in a population whose evolution is being simulated. Selection for reproduction in each successive generation is a function of the "fitness" of each individual relative to the rest of the population. "Fitness" is a measure of the relative score an individual gets oin some mathematical function intended to represent natural selection processes.

When a GA is used as an optimization tool, an optimization problem is defined by the objective function, which serves as a selection or "fitness" function. The potential solutions to the optimization problem are represented as simulated genotypes, and the "fitness" function is used to select relatively better genotypes and discard poorer ones, until a relatively optimal set of potential solutions is identified. In Nature, evolution may be viewed as an ever-changing optimization problem that requires acquiring resources such as food that are necessary for living long enough to rear viable offspring. Because of the growing recognition that nature's optimization methods $/$ can be used computationally by humans, GAs are now being widely used as extremely 
powerful global optimization tools for complex nonlinear problems in many fields, including business, economics, and engineering. In these applications, a genotype represents a point in the domain of a function being optimized. The value of the function at that point is treated as the individual's "fitness." The points that correspond to higher values of the function being optimized are the most "fit" and thus eventually come to dominate the population.

In this work, the GA is used a computational tool for simulating evolution rather than as an optimization tool. The "fitness" function is not some constant function being maximized (or minimized) as it would be in an optimization problem, but is instead a computational tool for simulating a selection process. Payoffs received by the DMs are used as the selection mechanism. Because the actual payoffs depend on the state of the commons and the behavior of the DMs, DM characteristics that might be optimal when the commons is in one condition may not be as optimal when it is in a different condition. The definition of "optimal" changes as the commons changes, and so the GA is generating the dynamics of the simulation, rather than solving an optimization problem.

In this $\mathrm{GA}$, each new iteration is formed by a simulated lottery process ("Lottery selection for reproduction" in the "Genetic Algorithm" part of Figure 6.1). In this lottery, pairs of genomes ${ }^{2}$ are randomly selected (with replacement) from the

\footnotetext{
${ }^{2}$ A genotype is a particular, unique combination of alleles, but a genome could be any genotype. In the case where there are eight possible combinations of alleles, there are eight genotypes. The number of genomes is the population size.
} 
current population $P o p_{i}$, and their payoffs are compared. The genome with the higher payoff in each pair has a higher probability TSProb of being copied to the pool of genomes from which the new population $P_{o p_{i+1}}$ will be constructed (Riolo, 1992). If TSProb is 1.0, then the higher-"fitness" member of each pair is always copied to the next iteration's progenitor pool; if TSProb is less than 1.0, then a larger stock of lower"fitness" members is more likely to be retained. Note that these genomes are not the new population, but are instead the progenitors of the new population. Genomes are selected and discarded in this way until the desired population size is reached. There are other ways of forming the progenitor pool which may be better for more complex problems (Goldberg, 1989), and this simple method might not have worked so well if there were more than three genes with two alleles each (Riolo, 1992). This algorithm was simple to use and worked well here, however. In particular, the ability to maintain diversity via the TSProb parameter turned out to be very important to the results (see Chapter VII).

In the next step, pairs of genomes from the new progenitor pool are again randomly selected, this time without replacement. Each gene in each genome is subjected to the possibility of mutation, where the value of the gene is randomly "flipped" (i.e., from 1 to 0 or vice versa), with some low probability $m r$ (e.g., 0.001). Next, a crossover site along the binary string representing the genome is randomly selected, and the two individuals swap genome segments at this crossover point. For example, if the first progenitor of a pair is represented as $\{110\}$ and the second 
progenitor is $\{011\}$, and the crossover point is between the second and third positions, then the two new individuals are $\{111\}$ and $\{010\}$. The progenitors themselves disappear; they are all replaced with their offspring.

It is this process of recombination that gives GAs their computational power (Holland, 1992). The crossover operator creates new combinations of genes each iteration, although when there are only three genes to a genome, and two alleles per gene, there is not a lot of variety to rearrange. Groups of alleles ("schemata") that are particularly advantageous represent partial solutions to the "fitness" problem, and, particularly if the genes are adjacent in the genome, tend to occur more often than less advantageous schemata. For example, in the example of the previous paragraph, if genotypes with the schema $\{11 * *\}$ tend to have higher average "fitness" than $\{01 *\}$ (the * is a wildcard character that means the bit could be 0 or 1 ), then $\{11 *\}$ will tend to occur more often in successive iterations.

In the computer code, each genotype consists of a binary array in which each variable corresponds to some component of the DM's decision function such as a probability table or an attribute weight. At each iteration, the algorithm decodes the genotype array to obtain the necessary decision variables, which it then uses to form multi-attribute value functions (described later in this chapter, in the "Decision Model" section) on which the choice is made. The magnitude of the payoff a DM receives, relative to the rest of the population, determines the probability that a particular DM genotype will be selected as a progenitor to the next iteration, and thus will be 
subjected to mutation and recombination. As the iterations pass, schemata that are particularly advantageous appear more often in the population. The definition of "advantageous" is a moving target, however: genotypes that are relatively successful when the commons is in good shape may not be so successful when the commons is degraded.

This is an important feature of this GA: the "fitness" function itself changes over time. The actions of the DMs impact the commons, and the commons provides the payoffs. However, the commons also changes because of its stochastic regeneration. Payoffs are not fixed by some explicit "fitness" function as when GAs are used for optimization. When GAs are used for optimization problems, the "fitness" functions are fixed, known, mathematical functions. In contrast, Nature's "fitness" functions are implicit; that is, they emerge over time from the interactions among the living members of an ecosystem, and between those members and their environment. The selection process in this study is more nearly implicit than selection processes used in most artificial life simulations, because of the feedback loop between the DMs and the commons. As a result, the most "fit" genotypes at one iteration may not be the most "fit" at a later iteration, because the commons will have changed; in a sense, the "fitness" function also evolves. 


\section{THE VILLAGE MODEL}

The village model consists of only one calculation, namely, exponential population growth. The exponential growth function for a population Pop at time $t$, with a growth rate of $G$, is (Gillett, 1981, 361):

(6.4) $\operatorname{Pop}(t)=P o p(0) * \exp (G t)$

Exponential population growth is discretized in the simulation by defining the population of interest as the maximum total number of potential Takers, at any iteration $i$, to be Tmax $_{i}$ (this assumption is discussed in the "Decision Model" section of this chapter) and assuming the time elapsed during an iteration is $\Delta t$. Then Eq. (6.4) becomes:

(6.5) $\operatorname{Tmax}_{i+1}=\operatorname{Tmax}_{i}+\operatorname{Tmax}_{i} * \exp (G \Delta t)$ 


\section{THE DECISION MODEL}

Seven factors determine a DM's choice: the decision rule, decision attributes, single-attribute value functions, attribute weights, beliefs, and the multi-attribute yalue function (von Winterfeldt and Edwards, 1986). In this model, all DMs use maximization of expected multiattribute value (EMAV) as their decision rule. Their single-attribute value functions, attribute weights, and beliefs vary according to their genotypes.

The first step in developing an EMAV model is to determine the attributes that best reflect the values of the DMs, and determine the appropriate mathematical form for each single-attribute value function. The next step is to derive weighting factors for the attributes in order to make them commensurable. Finally, a combining rule is used to compute the total value of the weighted single-attribute functions (Keeney and Raiffa, 1976). Von Winterfeldt and Edwards (1986) recommend starting with the simplest possible functions, only adding more complexity if sensitivity analyses reveal a need to do so.

For this research, the decision attributes must represent not only the selfinterested profit motives acknowledged in Hardin's metaphor, but also more socially or morally responsible values as well. 


\section{Pure self interest: profit}

Hardin said that a herdsman's only payoff is the proceeds of sale, and the extraction costs are negligible because they are shared by many (Hardin, 1968). The pure self-interest or "profit" attribute $\pi$ represents Hardin's assumed payoff. $\pi$ is a simple supply-dependent marginal profit function reflecting declining marginal returns.

Although Hardin did not include any modifications, in this model the marginal profit function can be modified by subtracting extraction costs, taxes, or penalties from the sales price. In the base model it is assumed that each Taker is successful and catches one unit of fish per iteration, and so the supply $T$ available to consumers is always equal to the number of Takers. In the base model, marginal profit $d \pi d T$, or $\pi$, is a per-unit function that is linearly proportional to the supply $T$ :

$$
\pi^{\prime}(T)=\pi_{\max }^{\prime}\left(I-T / T_{\max }\right)
$$

where $T_{\max }$ is the maximum possible supply, equal to the maximum possible number of Takers.

Marginal returns are assumed to be declining, and so as the number of fish harvested goes up, the price per fish goes down (see Figure 6.3). The slope is $-\pi^{\prime}{ }_{m a x} / T_{\max }$ where $\pi_{\max }^{\prime}$ is the maximum possible marginal profit and $T_{\max }$ is the total number of possible fishers. The magnitude of this slope is the profit function parameter $k$.

If the entire population of potential fishers $T_{\max }$ chooses to Take, the market is 
assumed to be saturated. Each can then receive only the minimum profit, which is zero: $\pi^{\prime}\left(T_{\max }\right)=0$. If none of them chooses to Take, the potential profit any one of them could get if they did Take (i.e., the marginal profit for $\pi^{\prime}\left(0^{\prime}\right)$ ) would be the maximum $\pi_{\text {max }}^{\prime}$. The maximum per-unit profit is determined by the slope of the marginal profit function and the total population of potential fishers $T_{\max }$.

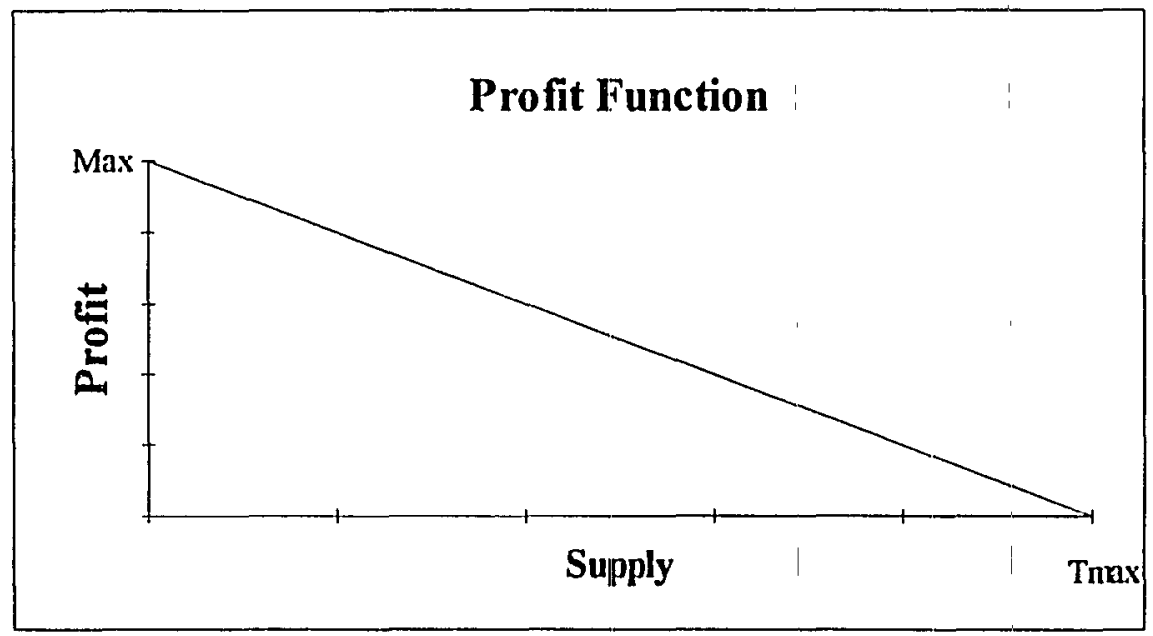

Figure 6.3. Marginal profit as a linear function of supply. The maximum supply is equal to the maximum possible number of Takers, $T_{\text {max }}$, which occurs when all the potential fishers choose to Take.

Each DM's total profit per iteration $\pi(I)$ is the marginal profit $\pi^{\prime}(T)$ times the number of units taken. Unless otherwise specified, the number of units Taken by a particular DM is always assumed to be one. Thus, $\pi(T)=(1) \pi^{\prime}(T):=\pi^{\prime}(T)$.

The total population consists of potential fishers as well as potential consumers, and so demand for fish grows as population grows. In a real village, the number of potential fishers may or may not increase proportionately to the nurnber of consumers, 
and it is difficult to justify any particular assumption about their relative growth. In the absence of any compelling reason for assuming otherwise, it is simplest to assume that the increase in the number of potential fishers is proportionate to the increase in number of consumers. With this assumption, the requirement that the market is saturated when all the potential fishers choose to Take is consistent for all population sizes, and so the marginal price sinks to zero at the right hand side of the graph (Figure 6.3) for any population size. However, even given this simplifying assumption, there are still two questions: what happens to the slope, and what happens to the $y$-intercept, of the marginal profit function when population increases?

While it does seem safe to assume that demand increases with increasing population, the question is how increasing demand should be represented with the marginal profit function. The marginal profit function slopes for different population sizes do not have to be parallel, or even linear, but it would be difficult to argue that the slopes would not be negative (i.e., that marginal price would not decrease with increasing supply). Three possibilities seem most reasonable: (1) a single marginal profit function for all population sizes, (2) constant slope but different $y$-intercepts, or (3) constant $y$-intercept but different slopes.

\section{Single marginal profit function for all populations}

It could be assumed that there is some maximum possible population Pop $_{\max }$ beyond which the potential fisher population could not grow, and that there is only one profit function that covers all possible population sizes. Then at any population size 
$T_{\max }<P o p_{\max }$ the requirement that the market be saturated when all potential fishers in a particular population decide to Take $\left(\pi^{\prime}\left(T_{\max }\right)=0\right)$ would be eliminated. The market would only be saturated if all the potential fishers in the maximum possible population Pop $p_{\max }$ decided to Take $\left(\pi^{\prime}\left(\right.\right.$ Pop $\left.\left._{\max }\right)=0\right)$. Such a marginal profit function would look like Figure 6.3 , but the equation of the marginal profit function would be slightly different because $T_{\max }$ would be replaced with Pop $_{\max }$ :

$$
\pi^{\prime}(T)=\pi_{\max }^{\prime}\left(I-T / P o p_{\max }\right)
$$

\section{Constant slope for all population sizes}

Another approach is to assume that as the population grows, the slope remains the same, and the market is saturated when all potential fishers Take, for any population size. In this case, the $y$-intercept $\pi_{\max }^{\prime}$ must increase as the population of potential fishers grows. Each population size would have its own, parallel, profit function with the same slope $k=k_{0}$ but a different $y$-intercept $\pi_{m a x}^{\prime}$. In other words, as the population grows, the curve moves out, away from the origin, as shown in Figure 6.4 .

In this case, the equation for the marginal profit function becomes:

(6.8) $\pi(T)=k_{0}\left(T_{\max }-T\right)$ where $k_{0}=\frac{\pi_{\max }}{T_{\max }}=$ constant

In Eq. (6.8), since the slope $k_{o}$ is constant, as the number of potential fishers 
$T_{\max }$ increases, so must the $y$-intercept $\pi_{\max }^{\prime}$. The marginal profit function is a function only of the (constant) slope and $\left(T_{\max }-T\right)$, which is the total number of Refrainers.

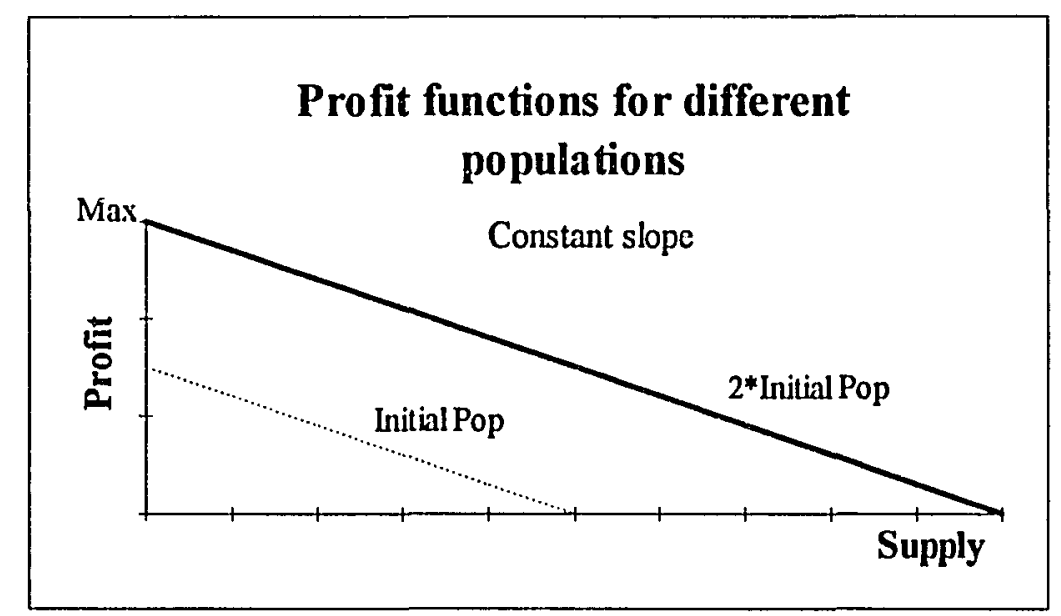

Figure 6.4. Marginal profit as a linear function of supply, at two population sizes, with constant slope. The dashed-line graph is for the lower, initial population size. The higher population size is twice that. The profit is zero when the supply is equal to the maximum possible number of Takers for a given population size, which occurs when all the potential fishers choose to Take.

\section{Constant y-intercept for all population sizes}

A third way to handle profit functions for different population sizes is to allow the slope to change so that the $y$-intercept remains a constant $\pi_{\text {max }_{o}}^{\prime}$ (see Figure 6.5). In this case, the maximum marginal price does not change with population size.

The equation for the marginal profit function is then the same as Eq. (6.6), but the difference is that $T_{\max }$ increases over time:

$$
\pi^{\prime}(T)=\pi_{\max _{0}}^{\prime}\left(1-T / T_{\max }\right)
$$


There are two significant differences between Eq. (6.9) and Eq. (6.7). First, in Eq. (6.9), the maximum possible profit is proportional to the fraction of the current population that decides to Take, whereas in Eq. (6.7), the maximum possible profit is proportional to the fraction of the maximum possible population that decides to Take. Second, in Eq. (6.7) the market is only saturated if the village is at its maximum population and all potential fishers decide to Take, whereas in Eq. (6.9) the market is saturated when all the potential fishers in any size village decide to Take.

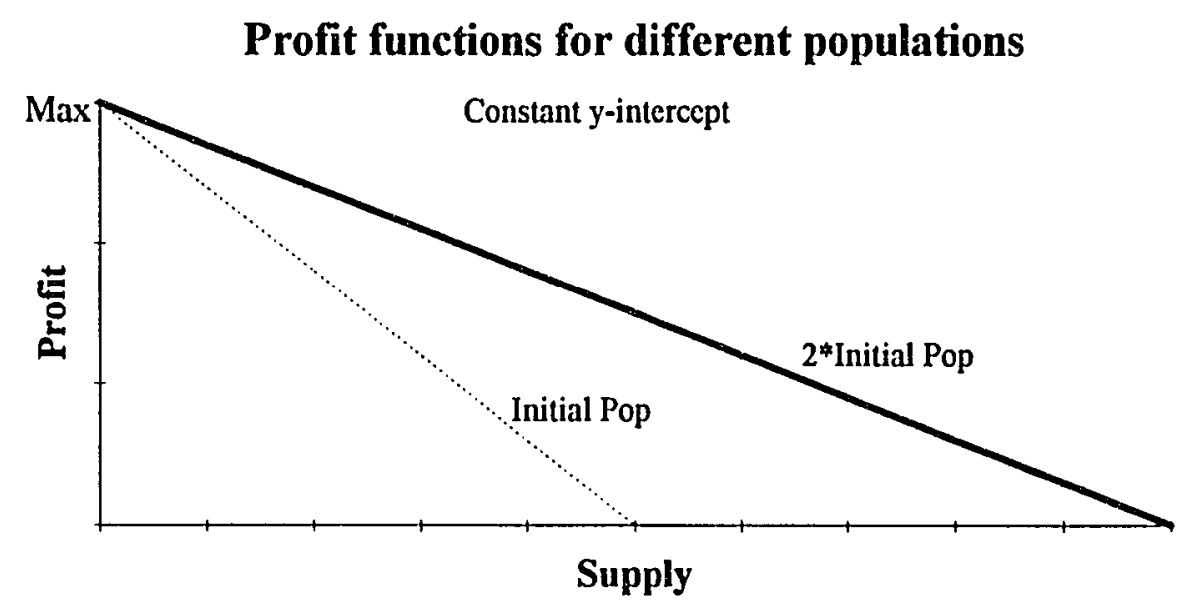

Figure 6.5. Marginal profit as a linear function of supply, at two population sizes, with maximum marginal profit held constant. The dashed-line graph is for the lower population size, and the higher population size is twice that. The profit is zero when the supply is equal to the maximum possible number of Takers for a given population size, which occurs when all the potential fishers choose to Take.

The marginal profit functions shown above are essentially demand curves, although in an economics text, supply is assumed to be a function of price (Gwartney and Stroup, 1980), rather than the other way around as it is here. If the market is 
always assumed to be at equilibrium, then the distinction does not matter.

The components of profit — prices and costs - are treated rather cavalierly here because it is simply assumed that per-unit (marginal) profit (per-unit price minus per-unit costs) increases linearly as supply drops. Extraction costs are not in general a direct function of the number of Takers, as price would be, but are more ordinarily a function of the state of the commons; there is no simple relationship between profit as a function of number of Takers, and extraction costs. Hardin treated extraction costs as negligible (Hardin, 1968). Here, costs are assumed to be incorporated into the marginal profit function.

The decreasing slopes in any of the marginal profit functions shown above imply that the marginal price that consumers are willing to pay is determined by supply (the number of fish that are caught). However, the supply and demand relationship in this simulation is not quite that straightforward: the number of fish that are caught is determined by the various fishermen's decision trees, which include speculations about how many others will Take, and hence the profit the DMs will be able to get. In that sense, supply is determined by anticipated demand. In these decision trees marginal profit is not the only attribute, however, because these potential fishers also may consider other attributes such as the one called "Conscience", which is not discussed in basic economics textbooks. The relationship between profit and supply is thus circular in the model, as it generally is in the real world.

All the marginal profit curves shown above have constant slopes, which means 
that demand is relatively more elastic in the high price range than in the low end: when an already-high price goes up, consumers are more sensitive to unit price changes and will tend to switch to substitutes or otherwise refrain from consuming that resource. If there are a lot of fish on the market and the price is low, consumers are more accepting of unit price changes and will pay the increased price. In general, the more vital a resource is perceived to be, relative to consumers' ability to pay (e.g., ernergency medical care or drinking water), the less elastic its demand (Gwartney and Stroup, 1980). If fish are the only source of protein in a village, the slope of any of the curves above will be nearly vertical because the underlying demand curves are relatively inelastic; but if lots of substitutes are easily available, the slope of the demand curve, and also the marginal profit function, will be relatively flat ${ }^{3}$.

A final complication in the single attribute value function for profit is that because subjective marginal value tends, for many people, to decline as the objective value increases for most goods (Gwartney and Stroup, 1980; Keeney and Raiffa, 1976), the subjective value of a particular profit to a particular DM could be different from the monetary value. Hardin ignored this effect because he assumed that the value of each additional unit was always +1 . If the subjective marginal value of profit were declining (e.g., the DMs were risk-averse), then the subjective profit functions would be as shown in the lower line of the graph in Figure 6.6.

\footnotetext{
3 "Flat" to an economist means parallel to the vertical axis; but to everyone clse, it neans parallel to the horizontal axis, and that is what it means here.
} 


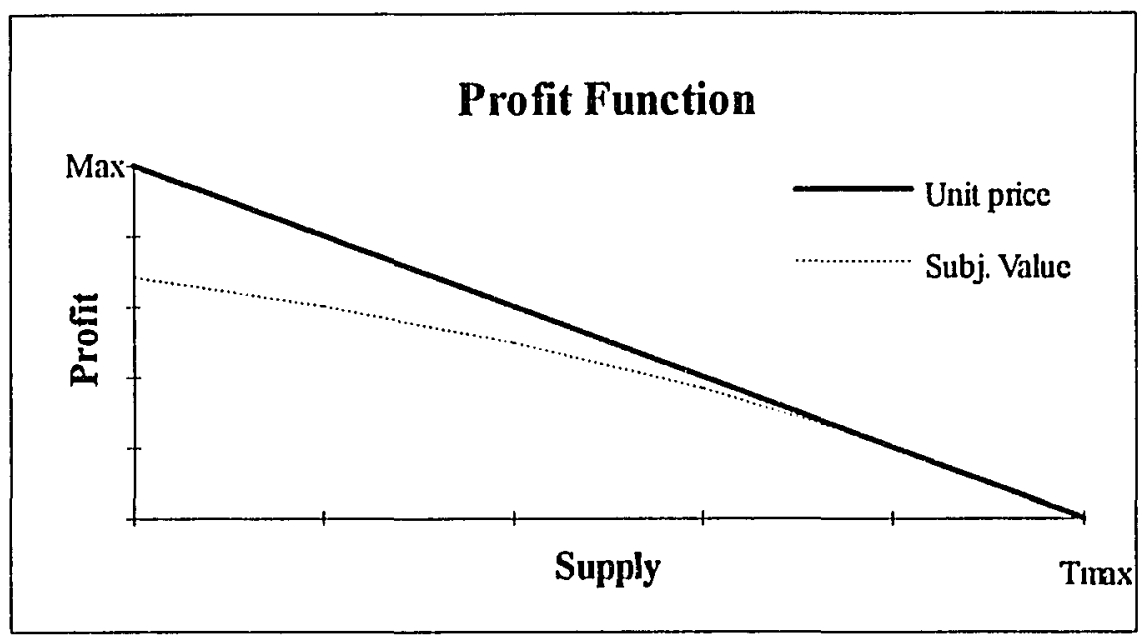

Figure 6.6. Subjective marginal profit function (dotted line) and objective value function (solid line).

The subjective marginal profit value function, which includes declining marginal value (shown with the dashed line in Figure 6.6) is related to the objective marginal profit function by (Keeney, 1992, 143):

(6.10) $\operatorname{SMV}\left(\pi^{\prime}\right)=\alpha-\beta\left(\exp \left(-\gamma \pi^{\prime}\right)\right)$

where $\alpha$ and $\beta$ are constants required to normalize the marginal profit function $\pi^{\prime}$, and $\gamma$ determines the rate of decrease of the subjective value function $S M V$ with increasing marginal profit $\pi^{\prime}$

Any of the three potential ways of handling the marginal profit function discussed above can be modified to account for declining marginal value. The question remains, however: Which of the marginal profit functions for growing populations is the most realistic? The advantage of assuming constant slope as in Figure 6.4 is the consistency of functional form across population sizes. In addition, both a basic 
economics textbook (Gwartney and Stroup, 1980) and a handbook on economic analysis of biodiversity (Pearce and Moran, 1994) assume the constant slope version (Figure 6.4); neither even discussed any other possibility for population growth. Because of this, as well as the consistency of form advantage, the assumption of constant slope (Figure 6,4) was used in the simulations unless otherwise specified.

Fortunately, as it turns out, which of the above formulations is used does not matter to the ultimate conclusion, and whether or not declining SMV is included does not matter either; all that matters is the assumption that demand grows with increasing population, and that it is subject to declining marginal returns.

\section{Conscience}

The second attribute value function in the decision trees is called the "Conscience" function $C(x) . C(x)$ is $\mid$ a simple linear function that rewards some DMs for Refraining and/or penalizes them for Taking. It represents concerns for the rights and needs of other people and/or other species, now and in the future.

"Conscience" is expressed as a linear per-unit or marginal function $C^{\prime}(x)$, where the conscience parameter $m$ determines the slope (see Figure 6.7):

(6.11) $C^{\prime}(x)=-m x+C^{\prime}{ }_{\max }$

In each iteration, each DM decides whether or not to Take one unit of commons, and so the total "Conscience" payoff per DM $C(x)$ is the per-unit payoff $C^{\prime}(x)$ times the number of units Taken ar Refrained. Because it is assumed that the decision is whether 


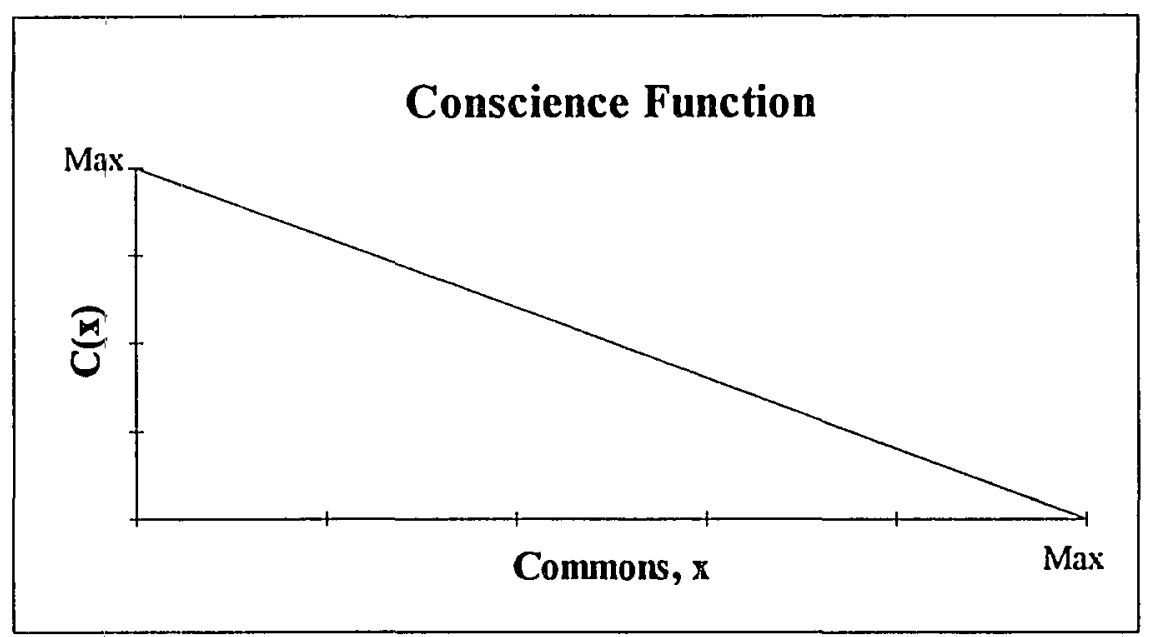

Figure 6.7. Marginal "Conscience" function. The state of the commons is $x$.

or not to Take one unit of commons, $C(x)$ is simply $1.0 * C^{\prime}(x)$ (note that $x$ is the state of the commons, not the number of units Taken). This makes the distinction between $C(x)$ and $C^{\prime}(x)$ computationally irrelevant, and thus for convenience the $C^{\prime}$ notation will be dropped. The theoretical distinction between a marginal attribute and a nonmarginal attribute is significant, however, because nonmarginal attribute value functions do not depend on the DM's choice and thus may drop out of decision trees ${ }^{4}$. If $C(x)$ were independent of choice (i.e., if it were a nonmarginal attribute), only the profit function would be represented in the decision trees and the role of social or moral considerations would be overlooked in the simulations. It is also difficult to imagine a "Conscience" function that rewarded or penalized a person for the ethical elements of a decision, but did not depend on that person's choice.

\footnotetext{
${ }^{4}$ Nonmarginal attributes drop out of decision trees only if the event nodes are also conditionally independent of the choice, as they are in this work; otherwise they may not.
} 
Note in Figure 6.7 that the magnitude of $C(x)$ increases as the commons becomes degraded. No DM feels particularly guilty about Taking (or virtuous about Refraining) if the commons is in excellent condition; when $x$ is at its maximum, $K$, then the marginal conscience function is assumed to be zero. Thus, since $m=C_{\max } / K$ : (6.12) $C(x)=m(K-x)$

The negative slope of $C(x)$ means that each commons unit becomes more important as the total number of them decreases. This is consistent with the way North American law and social mores apparently work: more resources are invested in trying to save or protect species that are perceived to be threatened or endangered, and there are often penalties for harming them as well as rewards for helping them. The moral and legal implications of killing a sockeye salmon in Idaho, where they are severely endangered, are more severe that they would be for killing a sockeye salmon in Alaska, where they are still plentiful.

Modeling conscience as a linear mathematical function that depends only on the state of the commons and the DM's choice is a great simplification of the kinds of things people take into account that might be grouped under "Conscience," as well as of the shapes different people's "Conscience" functions might have. Conscience could include judgments about distributional equity, both inter- and intra-generational, and even judgments about the rights of other organisms. Etzioni urges that economists acknowledge the "I\&We" dimension of values, which takes into account the weight that people put on responsibilities to others (Etzioni, 1988). Some environmental 
ethicists hold that human economic activities that lead to species extinctions are morally wrong (Ehrenfeld, 1981). A religious argument can be made either in favor of Taking or in favor of Refraining, depending on which part of the Bible one favors: Ehrenfeld, for example, argues that God gave humans the responsibility to care for His creation, but the Wise Use movement argues that God gave humans the responsibility to exploit His creation for their own ends (Ehrenfeld, 1981). To them, virtue would accrue for Taking and guilt for Refraining. Such a possibility is not included in the DM typology here: virtue is assigned to Refraining but not to Taking. The discussion about conscience-related solutions in the literature indicates that virtue applies to Refraining, and that is the assumption here as well.

Judgments about the morality of an act could also depend on what other people do. This would produce a "Conscience" function $C(x, T)$ that is dependent on the state of the commons $x$ as well as the number of Takers T. Such a function would be even more problematic to construct than the simple one shown in Figure 6.7, however. For example, one kind of person might believe that if most others are going to Take, then it is morally acceptable to go along with the crowd even if the commons is endangered; but another person might conclude that it is even more important to Refrain if others are going to be so irresponsible. Would $C(x, T)$ increase or decrease with the number of Takers? Hardin argued that it is the condition of the commons that determines the morality of an act: "the morality of an act is a function of the state of the system at the time it is performed. Using the commons as a cesspool does not harm the general 
public under frontier conditions, because there is no public; the same behavior in a metropolis is unbearable" (Hardin, 1968, 22, italics in original). To keep the model simple, Hardin's assumption is maintained here: the "Conscience" function is only a function of the state of the commons (and the DM's choice).

Because the "Conscience" payoff reflects the moral considerations of a "do not Take" decision rule, and the DMs assume that their individual actions have negligible effect on the commons, $C(x)$ reflects a deontological principle. To a deontologist, killing one of the last Idaho sockeye salmon is morally wrong, and thus so is killing one of many Alaska sockeye salmon: the act itself - killing fish — determines the morality, not the result of the act or the state of the system. However, there is also a rule-based utilitarian aspect to this function because the magnitude of $C(x)$ does depend on the state of the commons, even if that state is independent of a particular DM's choice: the magnitude of the "Conscience" penalty (or reward) increases as its relative effect on the commons increases. In this sense, killing one of many Alaska sockeye salmon would not carry as much moral weight as killing one of the last Idaho sockeye salmon because the degree of harm that results from the act depends on the state, and vulnerability, of the system.

In addition to factors that might be included in a "Conscience" function, there are certainly other assumptions that could be made about its shape. For example, it could be argued that if the commons is in very bad condition, some people would feel that they may as well Take, since the commons is already ruined. They might also feel 
that if the commons is in very good condition, Taking a unit would not matter either; only somewhere in the middle would conscience matter to them. $C(x)$ might then be shaped like an inverted " $\mathrm{U}$," with the peak somewhere near $x_{\max } / 2$.

"Conscience" could also be a discontinuous step function, reflecting the idea that for $x$ greater than, say, $x_{\max } / 2$, a DM would not feel any particular guilt about Taking (or sense of virtue for Refraining), but below that threshold concerns about ruining the commons would increase. It could then increase monotonically over some range as $x$ declines, and possibly at the lower end of the commons condition, there could be another step (either up or down). The Endangered Species Act, for example, "kicks in" at two levels: when a species is classified as "threatened" (a small number of individuals remain), and when it is "endangered" (very few individuals remain). Species or populations that are in danger of meeting criteria for listing under the Endangered Species Act are sometimes classified as "sensitive," and receive increased funding and management efforts intended to prevent their falling into the "threatened" or "endangered" categories. The levels of funding and effort do not increase smoothly along a continuum from "sensitive" to "endangered," and the moral aspects associated with Taking or Refraining from Taking may not increase smoothly either.

Finally, $C(x)$ could be a higher-order or exponential function, either over the whole range of $x$ or over any segment of a discontinuous or nonmonotonic function. A case could be made for any of these possible formulations of conscience; this seems, in fact, to suggest a rich area for further research. For example, government 
funding levels for restoration efforts for species such as salmon could be analyzed, or decision-analytic value function elicitation methods could be used, to derive something like "Conscience functions" for a variety of people. Occam's razor as well as prescriptive decision theory (e.g., Keeney, 1992; von Winterfeldt and Edwards, 1986) suggest using the simplest reasonable representation, and evaluating the potential effects of using other functional forms via sensitivity analyses (discussed in Chapter VIII). The simplest reasonable form would probably be a declining linear function such as Eq. (6.11). It is probably impossible to come up with a universal scale for "Conscience", let alone to determine how people might trade off greed and guilt. It is not the purpose of this research to take on such a task, but only to include a very crude approximation of the way "Conscience" might be used to offset greed in Hardin's simple metaphor, and to investigate whether it can be used to dispute Hardin's argument that no solution, including "appeals to conscience," will work in the face of growing human population.

Whatever form or definition a "Conscience" function might take, to Hardin's herdsmen only profit mattered. In effect, their attribute weight for "Conscience" was 0 , and their attribute weight for profit was 1.0. In this model, as in Hardin's metaphor, this type of DM always Takes. In contrast, a hard core conservationist would give no weight to profit and full weight to "Conscience", thus reversing the attribute weights. This kind of DM always Refrains. Hardin's herdsmen are represented as "Type I" DMs (see Table I), and hard core preservationists are represented as "Type IV" DMs. 


\begin{tabular}{|l|l|l|}
\hline DM payoff Type & $\begin{array}{l}\text { Payoff for } \\
\text { Taking one } \\
\text { unit }\end{array}$ & $\begin{array}{l}\text { Payoff for } \\
\text { Refraining } \\
\text { one unit }\end{array}$ \\
\hline I. Hard core Taker & $\pi(T)$ & 0 \\
\hline II. DM with mild conscience & $\pi(T)-C(x)$, & 0 \\
\hline III. Temptable conservationist & $\pi(T)-C(x)$ & $C(x)$ \\
\hline IV. Hard core Conservationist & 0 & $C(x)$ \\
\hline
\end{tabular}

Table I. The four DM payoff Types and their playoffs. $\pi(T)$ is the profit payoff, and $C(x)$ is "Conscience" payoff for Taking or Refraining one unit. $C(x)$ represents guilt in the "Payoff for Taking" column, and virtue in the "Payoff for Refraining" column.

There are two intermediate DM Types along the continuum from the hard core Taker Type I, to the hard core Refrainer Type IV.|A Type II DM feels guilt for Taking but feels no particular virtue for Refraining. This DM's total payoff for Taking is decreased by an amount $C(x)$, but the payoff for Refraining is still 0 . A Type II will Take until the commons declines to a low enough level that greed is overcome by guilt.

Like the Type II's, Type III DMs feel guilt in the amount $C(x)$ when Taking, but they also feel virtuous for Refraining. Like a Type IV, a Type III is a preservationist, but unlike a Type IV, a Type III will be tempted away from the "virtuous" choice if the commons is in fairly good shape and/or thepotentiall profit becomes substantial.

A decision tree would indicate algebraically that Type III DMs place twice the weight on $C(x)$ as do Type II's, because from a decision tree viewpoint it does not matter whether the "Conscience" payoff for Refraining is subtracted from the Taking 
payoff or added to the Refraining payoff. However, if $C(x)$ were reflected only in Type III's payoff for Taking, it would mean that the actual payoff for Refraining would be the same for Types II and III. Since actual payoffs are used as measures of relative fitness by the GA, it does matter to the GA which side of the decision equation $C(x)$ is on.

Note that the numerical value of $C(x)$ at any iteration $i$ is the same for all four DM payoff Types; but whether that value $C(x)$ is subtracted from a DM's Take payoff and/or is added to the Refrain payoff depends on whether the DM is Type $j=\mathrm{I}$, II, III or IV. The DM Type is determined by two genes $g_{1}$ and $g_{2}$ in the genome ${ }^{5}$. The alleles for $g_{1}$ and $g_{2}$ correspond to the coefficients of $C(x)$ in the payoffs shown in Table I (see Table II). The coefficient of $\pi(T)$ is determined by adding $g_{l}$ and $g_{2}:$ if the sum is less than 1.0 , then the coefficient of $\pi(T)$ is 1.0 (Types I, II, and III); otherwise it is 0.0 (Type IV).

\begin{tabular}{|l|l|l|}
\hline DM payoff Type & $g_{1}$ & $g_{2}$ \\
\hline I. Hard core Taker & 0 & 0 \\
\hline II. DM with mild conscience & -1 & 0 \\
\hline III. Temptable conservationist & -1 & 1 \\
\hline IV. Hard core Conservationist & 0 & 1 \\
\hline
\end{tabular}

Table II. The four DM payoff Types and the alleles for the Iwo genes $g_{1}$ and $g_{2}$. The alleles correspond to the coefficients of $C(x)$ in Table $I$.

${ }^{5}$ There are three genes in the genomes. $g_{0}$ is the gene that determines whether the DM is an optimist $\left(g_{0}=1\right)$ or a pessimist $\left(g_{0}=0\right)$. 
To put this mathematically, at iteration $i$ the "Conscience" payoff $V_{c}$ can be expressed as a function of the state of the commons $x$, the DM Type $j$, the DM's alleles for genes $g_{l}$ and $g_{2}$ and the DM choice $z$ :

$$
V_{c}\left(x_{i}, j, z\right)=\left[g_{1}^{j} C\left(x_{i}\right)\right] z+\left[g_{2}^{j} C\left(x_{i}\right)\right]\left(g_{2}^{j}-z\right)
$$

where $z=0$ for Refrain, and 1,0 for Take.

\section{Nonconsumable value $N(x)$}

There is another attribute that is sometimes included in analyses of natural resources that have some intrinsic or nonconsumable value (Pearce and Moran, 1994; Stern, Dietz, and Black, 1985-86). A nonconsumable attribute could represent enjoyment from hiking through an old-growth forest or across an ungrazed meadow, or from simply knowing there are wild steelhead in a river; its magnitude thus depends on the state of the commons $x$, and the single attribute function could then be designated $N(x)$. The value of $N(x)$ is not a function of any one DM's choice, but rather of the choices made by a great many other DMs, because of their impacts on the commons. $N(x)$ available to a DM is thus the same, regardless of what that one DM does, and if the event nodes are also conditionally independent of the choice (as they are in this study) $N(x)$ drops out of the decision tree $(N(x)$ is a nonmarginal attribute). How this happens will be shown in Chapter VI, along with an extensive discussion about the implications of this claim. 
Aggregation of the single-attribute value functions

Although it could be argued that there may be a second-order interaction between the two attributes (profit and "Conscience") for some DMs, this interaction is ignored in order to keep the model simple. The aggregate or total value is a weighted additive sum of the single attribute value functions (von Winterfeldt and Edwards, 1986; Keeney, 1992). The aggregate value function $\bar{V}$ for each outcome $\left(T_{i}, x_{i}\right)$ at iteration $i$ for each DM Type $j$ and choice $z$ is:

$$
\bar{V}\left(T_{i}, x_{i}, j, z\right)=\left[w_{\pi}^{j} \pi\left(\Gamma_{i}\right)+g_{1}^{j} C\left(x_{i}\right)\right] z+\left[g_{2}^{j} C\left(x_{i}\right)\right]\left(g_{2}^{j}-z\right)
$$

where:

$T_{i}=$ the number of Takers in iteration $i$

$x_{i}=$ state of the commons in iteration $i$

$j=$ DM Type number I, II, III or IV

$w_{\pi}^{j}=$ the coefficient of the profit function: 1 for Type I, II, and III DMs, and 0 for Type IV

$z$ = choice: 1 for Take and 0 for Refrain

$g_{1}^{j}$ and $g_{2}^{j}=$ the genes for DM $j$ that correspond to the coefficients for $C(x)$

(Table II)

To illustrate, the results of using Eq. (6.14) to calculate the total payoffs $\bar{V}$ are shown in Table III. The payoffs are the same as shown in Table I. 


\begin{tabular}{|l|l|l|l|l|l|}
\hline Type & $g_{1}^{j}$ & $g_{2}^{j}$ & $w_{\pi}^{j}$ & $\begin{array}{l}\bar{V} \text { for Taking } \\
(z=1)\end{array}$ & $\begin{array}{l}\bar{V} \text { for Refraining } \\
(z=0)\end{array}$ \\
\hline I & 0 & 0 & 1 & $\pi\left(T_{i}\right)$ & 0 \\
\hline II & -1 & 0 & 1 & $\pi\left(T_{i}\right)-C\left(x_{i}\right)$ & 0 \\
\hline III & -1 & 1 & 1 & $\pi\left(T_{\nu}\right)-C\left(x_{i}\right)$ & $C\left(x_{i}\right)$ \\
\hline IV & 0 & 1 & 0 & 0 & $C\left(x_{i}\right)$ \\
\hline
\end{tabular}

Table III. Summary table illustrating the total payoffs $\bar{V}$ that result from Eq. (6.14). $g_{1}^{j}$ and $g_{2}^{j}$ are the genes for DM $j$ that correspond to the coefficients for $C(x)$. The coefficient for $\pi\left(T_{1}\right)$ is 1.0 if the sum of $g_{1}^{j}$ and $g_{2}^{j}$ is less than 1.0 (Types I, II, and III), and 0.0 otherwise (Type IV).

\section{Expected Value}

Recall from the influence diagram in Figure 3.2 that the payoff a DM receives is determined not only by the DM's choice, but also by events whose outcomes are uncertain when the choice is made. In an EMAV model, the Expected Value $(E V)$ of each decision option is calculated by using the expectation operator from statistics to find the expected, or average, result of any course of action. For example, for a given current state of the commons $x^{*}$, choosing to Take could result in any of four potential outcomes, depending on the state of the commons $\left(x^{*}=\right.$ "Good" or "Bad" $)$ and what other DMs do $\left(T^{*}=\right.$ "most Take" or "most Refrain") (see Figure 6.8). 
EXAMPLE DECISION TREE FOR A TYPE I DM

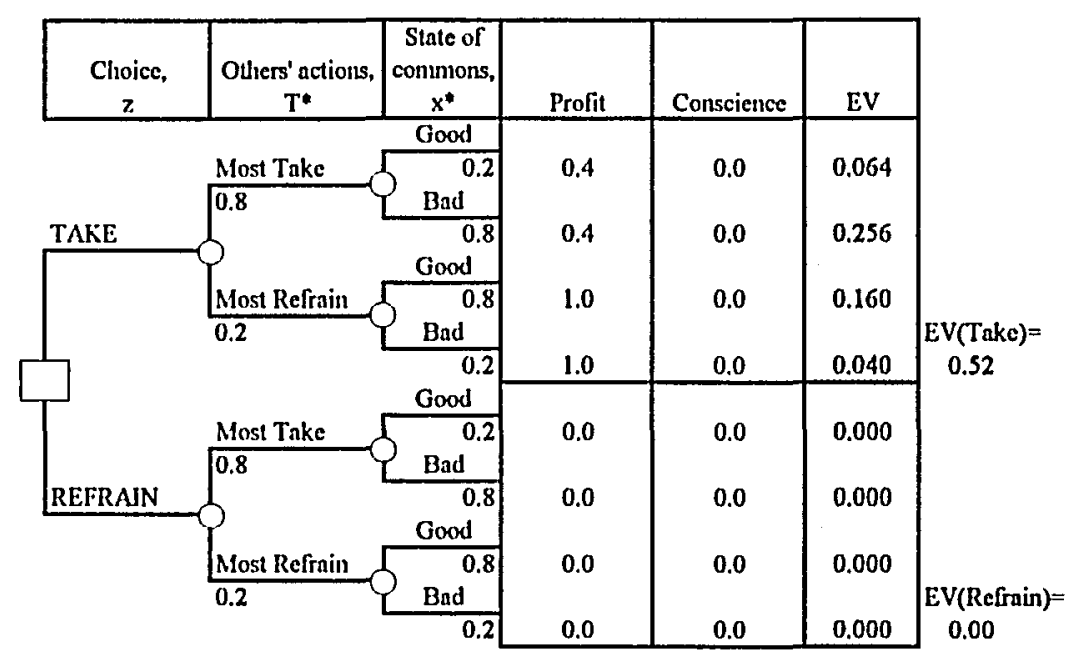

Figure 6.8. Type I or hard core Taker's decision tree.

The expected value of Taking is the average value of all the outcomes that might occur if the DM Takes. This average value is simply the sum of the aggregate values $\bar{V}$ for each of the four outcomes, where the number of Takers is $T^{*}$ and the state of the commons is $x^{*}$ (from Eq. (6.14)), each multiplied times the probability that it will occur.

For $\mathrm{DM} j$, the expected value $E V$ of choice $z$ is thus:

$$
\operatorname{EV}\left(T^{*}, x^{*}, j, z\right)=\sum_{x^{*}} \sum_{T^{*}} p\left(x^{*} \mid T^{*}\right) p\left(T^{*} \mid z\right) \bar{V}\left(T^{*}, x^{*} j, z\right)
$$

where:

$p\left(x^{*} \mid T^{*}\right) \quad=$ probability that the number of Takers will be $T^{*}$, given that the state of the commons is $x^{*}$

$p\left(T^{*} \mid z\right) \quad=$ probability that the number of Takers will be $T^{*}$, given that the 


$$
\begin{array}{ll} 
& \text { choice is } z \\
j & =\text { DM Type number I, II, III, or IV } \\
z & =\text { choice: } 1 \text { for Take and } 0 \text { for Refrain } \\
\bar{V} & =\text { aggregate value function (Eq. (6.14)) }
\end{array}
$$

Since the decision rule used by all these DMs is "maximize expected value," the DM chooses the option with the greatest $E V$.

To see how the value functions, attribute weights, and uncertainties are incorporated into the decision model, consider first the decision tree shown in Figure 6.8 , which reflects the values and beliefs of the Type I DM, who is a hard core Taker described by Hardin in the TOC (Hardin, 1968).

The oval event nodes from the influence diagram (Figure 3.2) have been replaced with circular event nodes in the branches on the left side of the decision tree in Figure 6.8. For the sake of simplicity, each event node is partitioned into two probability estimates, rather than being represented by a continuous distribution. DMs thus have to judge whether most DMs will Take or Refrain, and whether the state of the commons will be Good or Bad. They also have to define what they mean by "Good" or "Bad." Optimists expect fewer DMs to Take than do pessimists, and they expect the state of the commons to be better than pessimists do, regardless of whether it is Good or Bad.

The optimism or pessimism of these probability and outcome judgments is 
coded in the genotypes. If the value for the first gene in the genotype array $g_{0}$ is 1.0 , the DM is an optimist; if $g_{0}$ is 0 , the $\mathrm{DM}$ is a pessimist. Optimists and pessimists believe there are different probabilities asspciated with the event nodes shown in Figure 6.8 , as well as different outcomes $\left[T^{*}, x^{*}\right]$. A variety of levels for the magnitudes of the probabilities and the values used for $T^{*}$ and $x^{*}$ was investigated with the factorial experiments (see Chapter VII).

DMs base their estimates of what others will do $\left(T^{*}\right)$ on beliefs about how most people behave, and estimates about the state of the commons $\left(x^{\circ}\right)$ on the state of the commons of the last iteration. While it might have been more consistent to base expectations about how many others will Take on the last iteration, rather than on general beliefs about how people tend to act, doing so caused the simulations to oscillate too wildly. Basing estimates for $T^{*}$ on a more stable number $\left(T_{\max }\right.$ and a proportionality constant that varied with the genotypes) provided the "damping" necessary to solve the oscillation problem. This implies that, rather than having perfect knowledge about what others have done, and basing expectations on that, DMs stick to preconceived beliefs about how other people will act in the future, regardless of how they actually behaved recently. Sorne level of iearning is provided by the selection mechanism of the GA because it eliminates genotypes that are unreasonably optimistic or pessimistic.

Probability and outcome tables for optimists and pessimists in a typical simulation are shown in Table IV. For example, optimists believe that there is an $80 \%$ 
OPTIMISTS' PROBABILITY AND OUTCOME TABLE

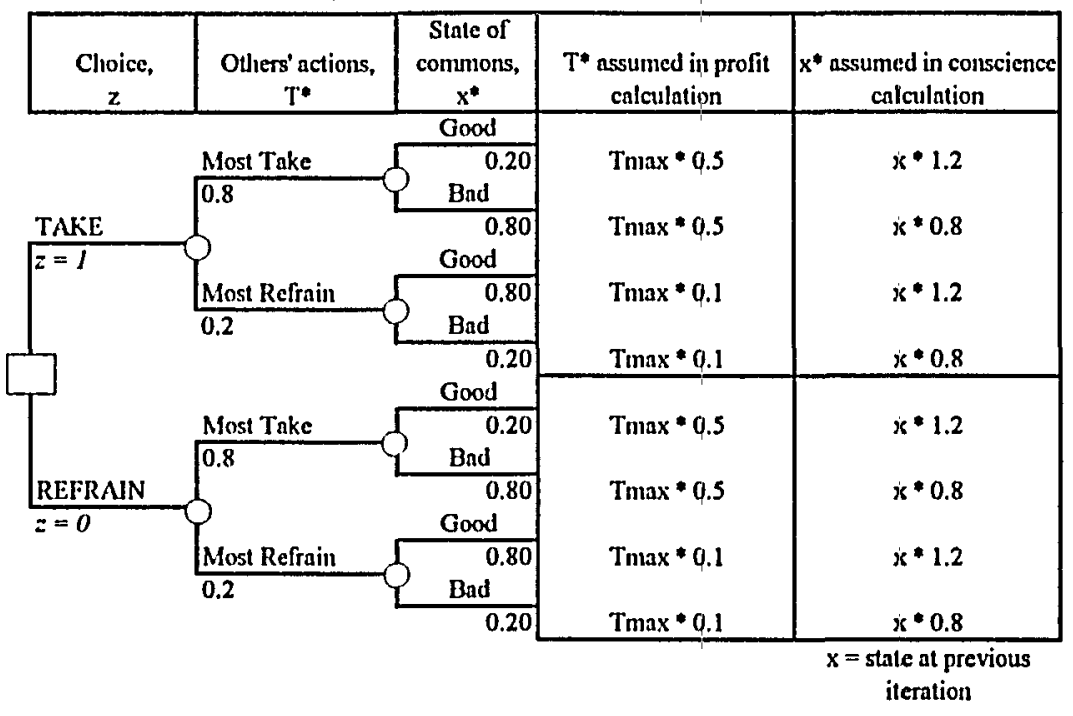

PESSIMISTS' PROBABILITY AND OUTCOME TABLE

\begin{tabular}{|c|c|c|c|c|}
\hline $\begin{array}{c}\text { Choice, } \\
z\end{array}$ & $\begin{array}{c}\text { Others' actions, } \\
T^{*}\end{array}$ & $\begin{array}{c}\text { State of } \\
\text { commions, } \\
\mathrm{x}^{*}\end{array}$ & $\begin{array}{l}\mathrm{T}^{*} \text { assumed in profit } \\
\text { calculation }\end{array}$ & $\begin{array}{c}x^{*} \text { assumed in conscience } \\
\text { calculation }\end{array}$ \\
\hline \multirow[b]{4}{*}{ TAKE } & & Good & \multirow[b]{2}{*}{ Tmax 0.9} & \multirow{4}{*}{$x * 0.8$} \\
\hline & Most Take & 0.05 & & \\
\hline & 0.9 & Bad & \multirow[b]{2}{*}{$\operatorname{Tmax} * 0.9$} & \\
\hline & & 0.95 & & \\
\hline \multirow[t]{7}{*}{$z=1$} & & Good & \multirow[b]{2}{*}{$\operatorname{Tm} \max 0.5$} & $x=0.5$ \\
\hline & Most Refrain & 0.50 & & $x * 0.8$ \\
\hline & 0.1 & Bad & & \multirow[b]{2}{*}{$x * 0.5$} \\
\hline & & 0.50 & $T \max * 0.5$ & \\
\hline & & Good & \multirow[b]{2}{*}{ Tmax 0.9} & \multirow[b]{2}{*}{$x * 0.8$} \\
\hline & Most Take & 0.05 & & \\
\hline & 0.9 & Bad & & \multirow[b]{2}{*}{$x * 0.5$} \\
\hline \multirow{2}{*}{$\frac{\text { REFRAIN }}{-=0}$} & & 0.95 & $T_{\max } * 0.9$ & \\
\hline & & Good & & \multirow[b]{2}{*}{$x \circ 0.8$} \\
\hline \multirow{4}{*}{$z=0$} & Most Refrain & 0.50 & Tmax $* 0.5$ & \\
\hline & 0.1 & Bad & & \\
\hline & & 0.50 & $T \max \cdot 0.5$ & $x \cdot 0.5$ \\
\hline & & & & $\begin{array}{c}\mathrm{x}=\text { state at previous } \\
\text { iteration }\end{array}$ \\
\hline
\end{tabular}

Table IV. Probability estimates and outcomes $[T, x]$ assumed by optimists and pessimists in the simulations. A variety of yalues for the probabilities and $T$ and $x^{*}$ were used in different simulations, but the tables shown here are typical. 
probability that the number of DMs who will Take will be $50 \%$ of the potential Takers (i.e., $T^{*}=0.5 * T_{\max }$, see the first row at the top of the Optimists' table in Table IV). They believe that there is a $20 \%$ chance that even if most others Take, the state of the commons will still be $20 \%$ better than the last iteration (i.e., $x^{*}=x_{i} * 1.2$ ). If instead most others Refrain, an optimist believes there to be an $80 \%$ probability that the state of the commons will be only $80 \%$ of the state at the last iteration (i.e., $x^{*}=x_{i}^{*} 0.8$ ). As discussed previously, these probabilities and outcomes are the same if the DM Takes or Refrains, because one of the fundamental nature of a commons dilemma is that actions by others, and the state of the commons, are negligibly affected by a particular DM's actions.

In contrast to optimists, in a typical simulation pessimists believe there is a better chance most people will Take: they estimate a $90 \%$ probability that $90 \%$ of the potential Takers will Take $\left(T^{*}=T_{\max } * 0.9\right.$; see the lower table in Table IV), and a $10 \%$ probability that $T^{*}$ will be $T_{\max } * 0.5$. If imost Take, a pessimist believes there is only a $5 \%$ chance that the commons will be in Good condition, where Good is only $80 \%$ of its previous condition $\left(x^{*}=x_{i} * 0.8\right)+$ in contrast to optimists, who think there is a $20 \%$ chance that the commons will be in "Good" condition, where they define "Good" to be $x^{*}=x_{i} * 1.2$.

An arbitrary normalized value function for profit $V_{\pi}$ is shown in the first column on the right side of the table in Figure 6.8. For this Type I DM, the highest $V_{\pi}$, 
1.0, is expected for choice Take, when the other DMs Refrain $\left(T^{*}=T_{\max } * 0.1\right)$. The state of the commons is irrelevant in this case because profit is only a function of the number of Takers and $C(x)$ is always zero for Type I's. Their worst payoff is if they Refrain, regardless of the state of the commons, because Type I's represent Hardin's herdsmen, who perceive no value in Refraining (Hardin, 1968).

The normalized value function for "Conscience" $V_{c}$ reflects what Type I DMs believe about right and wrong. The hard core Takers shown in Figure 6.8 have no "Conscience" and so $V_{c}$ is always zero (another way to put this, consistent with the earlier discussion, is that for Type I's, the coefficient for $C(x)$, as shown in Table III, is $0)$. As for the rest of their decision model, the Type I's shown in Figure 6.8 happen to be optimists, and so their probability and outcome estimates are as shown in the optimists' table in Table IV.

Eq. 6.14 is used to calculate the $E V$ for each possible outcome $l$, where the profit function $\pi(T)$ and "Conscience" function $C(x)$ are replaced with their normalized versions $V_{\pi}$ and $V_{c}$. For example, the $E V_{l}$ in Figure 6.8 when this optimistic Type I DM decides to Take, and expects most others to Take, and the state of the commons to be Good (first row in the $E V$ column), is:

$$
\begin{aligned}
E V_{l}= & \operatorname{Pr}(\text { Others Take }) * \operatorname{Pr}(\text { Commons is Good } \mid \text { Others Take }) *\left(V_{\pi}+\right. \\
& \left.V_{c}\right) \\
= & 0.8 * 0.2 *(0.4+0) \\
= & 0.064
\end{aligned}
$$


The calculation shown in Eq. (6.16) is performed for each of the four possible outcomes that could result for this DM if the choice is to Take. The results are added, to yield an expected value EV(Take) of 0.52 (Figure 6.8). An equivalent calculation is performed for the choice to Refrain, to yield an EV(Refrain) of 0.0 . Given a Type I's emphasis on profit, and lack of interest in "Conscience", as Hardin predicted, this DM has no choice but to Take a unit of the commons $(E V($ Take $)=0.52)$ rather than to Refrain (EV(Refrain) $=0.0)$.

As a contrasting example, consider the Type IV DM shown in Figure 6.9, who perceives no value in Taking $\left(w_{\pi}=0\right)$, but bases the decision instead only on the "Conscience" function. The Type IV shown in Figure 6.9 happens to be a pessimist. A pessimistic Type IV DM is more representative of a hard core preservationist, i.e., a pejorist in Hardin's terms (Hardin, 1977), and hard core preservationists always prefer to Refrain $(E V($ Refrain $)=0.28$ in Figure 6.9) rather than to Take $(E V($ Take $)=0)$.

To complete these examples, recall that where a DM falls along the continuum between the extremes illustrated by these two DMs is determined by that DM's genotype. One gene $\left(g_{0}\right)$ determines the DM's level of pessimism or optimism; each allele corresponds to a different table of probability estimates and expected outcomes (Table IV). The other two genes, $g_{l}$ and $g_{2}$, determine their profit and "Conscience" value functions (Table III). Just as was done in these two examples, decision trees for the remaining six DM genotypes are created by using the probability and outcome tables of Table IV, which depend on the allele for $g_{0}$; and the profit and "Conscience" 
payoffs of Table III, which depend on the alleles for $g_{1}$ and $g_{2}$.

EXAMPLE DECISION TREE FOR A TYPE IV DM

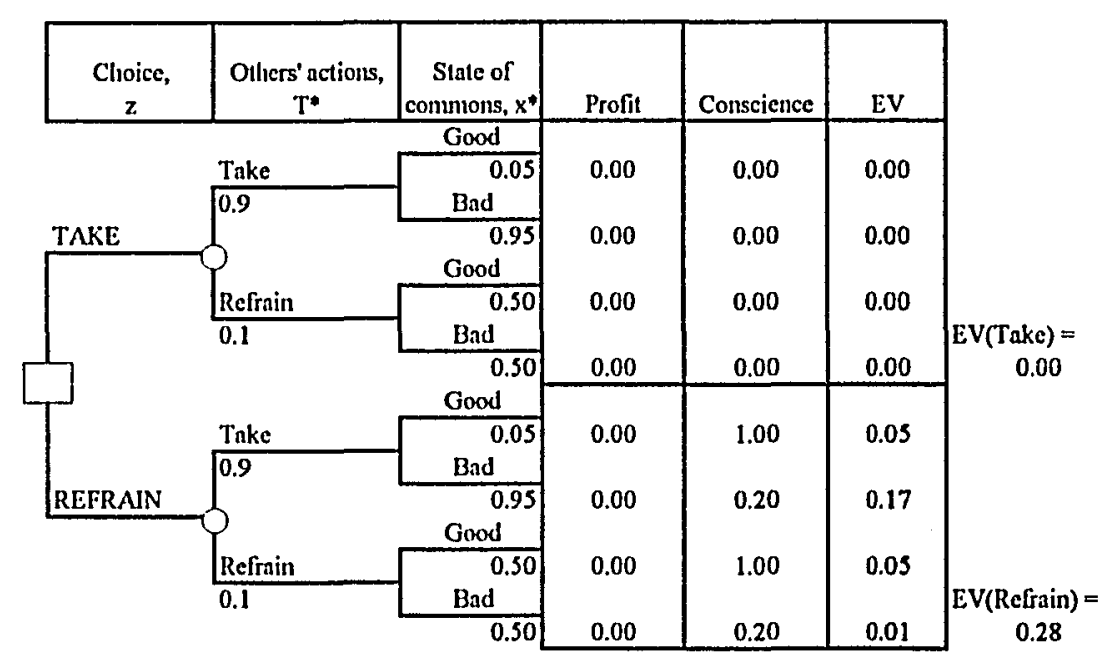

Figure 6.9. Type IV or hard core Refrainer's decision tree.

The contrast between the two DM extremes described above is particularly important because according to decision theory, both may be defined as "rational," in that they select the actions that they believe have the best chance of providing the most of what they value. Yet Hardin argued that only the self-interested herdsman meets the criteria of "rationality" (Hardin, 1968). If Hardin's simple model is indeed a realistic representation of common resource decision making, then the TOC is an inescapable dilemma with no solution. However, if including the impacts of beliefs about likely outcomes, or the importance of "Conscience", makes a model more faithful to the real world, then it may be that under some circumstances solutions are possible. The point is that when a more complete model of decision making is considered than can be represented by the archetype TOC, it may be possible for rational DMs to avoid 
destroying the commons. In that case, Hardin would be wrong. 


\section{FITNESS}

\section{The meaning of fitness}

The function that is used to measure relative "goodness" of the genotypes is the cornerstone of any evolutionary model and is usually called the "fitness function." In biology, fitness is the basis for reproductive selection and thus evolution. In this model, the only aspects of biology that are modeled are the regeneration rate of the commons and the population growth of the villagers. Nothing is implied about the genetics or biological evolution of the villagers; what "evolves" in this model is the distribution of beliefs and values of the villagers.

The simulated mechanisms of evolution in this GA are no more than mathematical devices for simulating social change. "Genes," which are used by the GA to represent beliefs and values, are simply binary elements of the computer code. What they represent is more like Richard Dawkins' "memes" (Dawkins, 1978) than like biological genes. Dawkins coined the word "meme" to represent a transmissible unit of social evolution analogous to a gene in bijological evolution. To Dawkins, memes are ideas. They can be transmitted from one person to the next, they can mutate in the process, and they are subject to something like recombination: in "memetic" evolution, mutation would be something like what happens in the game of "gossip," where the message received has some probability of being different from the message transmitted. 
In "memetic" evolution, recombination would be the means whereby new ideas are developed from combinations of elements from old ideas, or where people learn, or change their minds; mutation would be where they fail to communicate accurately.

Thomas Dietz has recently developed an idea of social evolution that fits the evolutionary framework of this model quite well (Dietz and Burns, 1992, 187):

By [social] evolution we mean models of the generation of variety, transmission of information and the action of selection and other processes (drift, migration, etc.) on the distribution of information within a population over time. When the information is contained in genes, this general model becomes the neo-Darwinian synthetic theory of evolution. When the information consists of social rules, the general scheme becomes a theory of sociocultural evolution. Macro or population-level phenomena and structures are shaped by micro-level processes and in turn are the selective environment for the micro-level process.

What Dietz means by "rules" is similar to what Dawkins means by "memes," and corresponds to what this work means by "genes" — i.e., beliefs and values used for making decisions and evaluating their outcomes (Dietz and Burns, 1992, note 3, 197):

Such rules specify what exists and, as a corollary, what does not exist, what nature, society, men and women are like. They shape images of what is real and true, what factors to take into account, and what to ignore (Czarniawska-Joerges 1988). Evaluative rules assign values to things, people, deeds, events and states of the world, defining what is 'good' or 'bad,' what is acceptable and unacceptable, what is right, just, beautiful, attractive, enjoyable as well as their opposites -- in general what people should strive for or avoid.

Dietz says that social evolution is a kind of learning process, in which an individual tries a rule, "assesses the results and retains, modifies or rejects the rule 
based on the outcome" (Dietz and Rosa, 1994, 190). Further, (Dietz and Rosa»1994, 190):

Social learning is the cultural analog of the genetic transmission process. Processes of transmission, selection, migration, drift, recombination and mutation will lead to changes over time in prevalence of rules in a culture...such changes are social evolution.

To Dietz, the selection process that drives social evolution is subjective. He envisions a selection process for these "rules," or beliefs and values, that is virtually the same as the GA selection process used in this work (Dietz and Rosa, 1994, 190):

Actions that implement rules produce responses from other actors and the ecosystem. Such responses, once perceived (using rules of interpretation) may cause an actor to modify or discard some rule or set of rules. In addition to direct experience in trying out rules, actors use the observed behavior of others and social discourse...to determine rules that are likely to produce desirable outcomes.

In a similar way, to Dawkins, the fitness of a "memotype," like the fitness of a genotype, is a measure of how prevalent it is in a population and thus how successful it is in taking hold within a population. Dawkins argues that memes that are evolutionarily successful, like genes that are evolutionarily successful, tend to propagate through a population more vigorously than memes that are less successful (Dawkins, 1978). Evolutionary success or "fitness" for a meme thus reflects the popularity of an idea in a population.

What "evolves" in this work is memotypes - those sets of values and beliefs, or what Dietz would call rules - that individual people might hold at any one time 
step. At the next time step, a real-world optimist might become a pessimist, or someone who had no "Conscience" might become concerned about the moral aspects of an action. These changes are represented by the "evolutionary" mechanism provided by the GA. It is not the human species that evolves in this model; it is beliefs and values held by a population of humans in a small village.

Evolutionary change in this model is driven by the actual value of the outcomes, measured on two attributes: profit, and "Conscience" "Conscience" functions are used to calculate the payoffs as are used in the decision trees (see Table I or III). However, whereas in the decision trees, the expectation operator from statistics (Eq. (6.15)) is used to estimate profit and "Conscience" payoffs by multiplying the estimated values of potential outcomes $\left[T^{*}, x^{*}\right]$ by the probabilities of their occurrence, the actwal payoffs are calculated by using the actual numbers of Takers and the actual state of the commons $\left[T_{i}, x_{i}\right]$ instead of the estimated numbers $\left[T^{*}, x^{*}\right]$. No probability estimates are needed for the actual payoff calculations because the outcomes are known (examples of "fitness" calculations are given below).

The actual payoffs are used as the selection mechanism ("fitness function") in the GA. Alleles that provide relatively greater payoffs become more prevalent than those that provide relatively lower payoffs at any particular time. If optimists get better

\footnotetext{
${ }^{6}$ It is important to cmplasize that these idealized DMs use the most widely accepted tool of prescriptive decision making, rather than the apparently haphazard approach ordinary humans ordinarily use. Assuming that everyone uses a prescriptive method for making decisionsl is a "best case" approach, and thus tends to bias the model towards showing that Hardin is wrong:i if even such careful DMs as these cannot escape the commons tragedy, it tends to support Hardin's thesis.
} 
payoffs than pessimists, there will be more optimists in the next iteration: more genotypes will have $g_{0}=1$ than $g_{0}=0$; if DMs who include a "Conscience" penalty for Taking $\left(g_{l}=-1\right)$ get better payoffs than those who do not $\left(g_{l}=0\right)$, more genotypes will have $g_{l}=-1$. The more popular decision models are thus the ones that are found to be more "fit" by the GA, by virtue of their resulting in greater payoffs. The selection process of the GA causes decision trees that provide greater payoffs to be more prevalent in successive iterations.

The state of the social ecosystem in this metaphorical fishing village is represented by the distribution of memes (per Dawkins) or rules (per Dietz): that is, the distribution of values and beliefs. Social evolution is then represented by the change in distribution over time of what is called genotypes in a GA. Here, evolutionary time is not measured by generations, as it would be in biology, but rather by an unspecified measure used as the iteration time unit $\Delta t$ of the discrete computer simulation. This simulation discretizes what are in reality three continuous processes: exponential population growth of the village, logistic regeneration of the commons, and the social evolution of beliefs and values in the village. The time step used by the discretization of the (continuous) logistic and exponential functions is simply one arbitrary nondimensional unit that is equivalent to the period of time between one discrete set of decisions by the villagers, and the next.

Using subjective rewards and penalties to drive an evolutionary model has a potential difficulty that arises from the problem of interpersonal comparison of value. 
No satisfactory way to compare one person's utiles to another has yet been established in decision theory. It is a great simplification to assume that, because a Type I DM's choice produces a net normalized payoff of 0.7 , while a Type III DM's choice yields 0.3 , that the Type I will become more popular in the next time step. A payoff of 0.7 on one person's scale might be less than 0.3 on someone else's scale, unless it is somehow known how much one unit is worth, on one person's scale, to someone else with a different scale. It is well established in decision theory that even as apparently objective a unit as a dollar is generally worth more to a person who already has only ten dollars, than to a person who already has a thousand dollars (c.f., Keeney and Raiffa, 1976). This was not a problem for Hardin's herdsmen, because Hardin simply assumed that each cow is worth " +1 ", on a scale he did not specify. In the real world, the relative value of a "cow-unit" would certainly be different for different people, and even for the same person in different circumstances. No such scale has ever been established. This is the source of the problem of interpersonal value comparisons.

For lack of any better approach available, the approach taken here is to assume interpersonal comparability, where the units are units of profit: consistent with Hardin, it is assumed that a unit of profit, for number of Takers $T$, is worth the same amount to anyone who values profit at all (i.e., Types I, II, and III DMs; to Type IV's, profit is worthless). However, inconsistent with Hardin, but consistent with simple economic models, the value of the profit function is not always " +1 ", but is $\pi(T)$ : it depends on how many units are on the market (i.e., the number of Takers $T$ ). Because the value of 
$\pi(T)$ is the same for all Type I, II, and III DMs, it means interpersonal comparability is assumed.

Similarly, interpersonal comparability also means that the value of the "Conscience" payoff $C(x)$ is also the same for all. The difference among the four DM Types is due to the coefficients associated with the profit and "Conscience" functions (Table IV). These coefficients can only be $0,-1$, and 1 , and so there are only a very few, limited, and primitive kinds of payoffs represented. However, Hardin assumed all villagers had exactly the same payoff function, with a value of +1 under all conditions, and he discounted any considerations but profit. While far from a perfect representation of real decision-makers, this model is still a step towards reality - albeit an idealized and simplistic reality - from Hardin's.

\section{Calculation of fitness}

The payoff received by each DM at the end of each iteration is determined by the actual number of Takers $T_{i}$, the actual state of the commons $x_{i}$ resulting from all the other DMs' actions, and by that DM's "Conscience" and profit function coefficients. The actual payoff a DM receives, relative to the payoffs received by the rest of the population, is used as the DM's "fitness function" for the evolutionary part of the model. The actual payoff is called the Realized Value $R V$. Recall that the decision trees use the expectation operator from statistics (Eq. (6.15)) to calculate Expected Value EV-i.e., the estimated outcomes based on judgments about how many Takers there 
might be $T^{*}$, and what the state of the commons might be $x^{*}$.

In contract to the $E V$ calculations used in the decision trees, the actual payoff calculations $R V$ do not use probabilities and judgments because the actual number of Takers and the actual state of the commons are known: $T^{*}$ and $x^{*}$ are replaced with $T_{\text {, }}$ and $x_{i}$, and $R V$ is calculated by using the aggregate value function $\bar{V}$ of Eq. (6.14).

To illustrate, consider the payoff received by a DM who is a "temptable preserver" or a Type III from Table I. Recall that for a Type III DM, profit is important but is diminished by a "Conscience" penalty for Taking; a Type III also gets a "Conscience" bonus for Refraining. Eq. (6.14) is shown again here for convenience, applied to a Type III DM:

$$
\bar{V}\left(T_{i}, x_{i}, j, z\right)=\left[w_{n}^{j} \pi\left(T_{i}\right)+g_{1}^{j} C\left(x_{i}\right)\right] z+\left[g_{2}^{j} C\left(x_{i}\right)\right]\left(g_{2}^{j}-z\right)
$$

where: $\quad j=\mathrm{DM}$ Type III

$$
\begin{aligned}
& z=\text { Choice, } 1 \text { for Taking and } 0 \text { for Refraining } \\
& g_{1}^{j}=\text { coefficient for } C(x) \text { for Taking for } \mathrm{DM} j=-1 \\
& g_{2}^{j}=\text { coefficient of } C(x) \text { for Refraining for } \mathrm{DM} j=1
\end{aligned}
$$

Using these values in Eq. (6.14) gives the Type III's actual payoff for outcome $\left(T, x_{i}\right)$

$$
\begin{array}{ll}
\bar{V}\left(T_{i}, x_{i}, I I I, I\right)=\pi\left(T_{i}\right)-C\left(x_{i}\right) & \text { for Taking: } z=1 \\
\bar{V}\left(T_{i}, x_{i}, I I I, 0\right)=C\left(x_{i}\right) & \text { for Refraining: } z=0
\end{array}
$$

If this Type III DM had decided to Take, the actual payoff $R V$ would be Eq. 
(6.17a); if this DM had decided to Refrain, the actual payoff $R V$ would be Eq. (6.17b).

Equivalent calculations are used for the other three Types of DMs. The payoffs received by all the DMs are used in the selection, mutation, and recombination steps of the GA discussed previously; thus, $R V$ is used as the "fitness" function in the GA.

To summarize the fitness calculation, the "fitness" function" used by the GA is:

$$
F_{i j}=R V_{i j}=\bar{V}\left(T_{i}, x_{i}, j, z\right)
$$

where $\bar{V}$ is the aggregate value function (Eq. (6.14)), applied for $\mathrm{DM} j$ in iteration $i$, who makes choice $z(z=1$ for Take and 0 for Refrain) when the actual number of Takers is $T_{i}$ and the actual state of the commons is $x_{i} . F_{i j}=R V_{i j}$ is used in the GA to select the progenitors for the population of the next iteration.

Now that all the components of the "fitness" function have been derived, it is possible to express the probability $\operatorname{Pr}$ of a particular genotype $j$, in iteration $i$, being a progenitor to iteration $i+I$ (and hence subject to mutation and recombination). This probability is a function $f$ of two things: the probability TSProb (see Section 3, earlier in this chapter) that the more fit member of a pair of genotypes will be selected to go through mutation and recombination; and the "fitness" function $F_{i j}$ :

$$
\operatorname{Pr}(j, i+I)=f\left[\operatorname{TSProb},\left(F_{i j} / \frac{1}{T_{\max }} \sum_{j} F_{i j}\right]\right.
$$

where $T_{\max }$ is the effective population size or number of potential Takers. 


\section{MATHEMATICAL SUMMARY OF MODEL}

Figure 6.1, at the beginning of this chapter, provided an overview of the way the four components of this model (the commons model, the decision models, the village model, and the GA) interact. The discussion that followed developed the model components of more rigorously, and it may be useful here to summarize the sub-models mathematically. The equation reference numbers are the same as in the text above.

\section{- Commons model}

\section{$\underline{\text { Nominal regeneration }}$}

$$
r\left(x_{i}\right)=r_{0} x_{i}-r_{0} x_{i}^{2} / K
$$

Nominal regeneration rate at iteration $i=$ logistic growth equation. $r_{0}$ is the regeneration rate constant, $x$ is the state of the commons, and $K$ is the commons carrying capacity or $x_{\text {max }}$

\section{State of the commons}

$$
x_{i+1}=x_{i}-T_{i}+N\left\{r\left(x_{i}\right), \text { Stoch } * r\left(x_{i}\right)\right\}
$$

State of the commons at itcration $i+l=$ state at iteration $i$ - depletion by Takers + stochastic regeneration. $N\left\{r\left(x_{i}\right)\right.$. Stoch $\left.* r\left(x_{i}\right)\right\}=$ sample from the normal distribution with mean $r\left(x_{i}\right)$ and slandard deviation Stoch $*\left(x_{i}\right)$.

\section{- Village model}

\section{Population growth}

(6.5) $\operatorname{Tmax}_{i+l}=\operatorname{Tmax}_{i}+T \max x_{i} * \exp (G \Delta t)$

Number of potential Takers at iteration $i+l=$ Number of potential Takers at iteration $i+$ exponential growth equation, with growth rate $G$, over time increment $\Delta t$. 
- Decision model

\section{Aggregate Value of payoff}

$$
\bar{V}\left(T_{i}, x_{i}, j, z\right)=\left[w_{\pi}^{j} \pi\left(T_{i}\right)+g_{1}^{j} C\left(x_{i}\right)\right] z+\left[g_{2}^{j} C\left(x_{i}\right)\right]\left(g_{2}^{j}-z\right)
$$

Aggregate weighted payof $=$ weighted Profit and weighted "Conscience" (guilt) payoffs for Taking $(z=1)$ or weighted "Conscience" (virtue) payoff for Refraining $(z=0)$. $w_{\pi}^{j}=1$ for all Types except IV, for whom $w_{\pi}^{j}=0 . g_{1}^{j}$ is the gene that determines the coefficient $(-1$ or 0$)$ for $C(x)$ for DM $j$ for Taking; $g_{2}^{j}$ is the gene that determines the coefficient $(0$ or 1$)$ for $C(x)$ for DM $j$ for Refraining.

\section{Expected Value of payoff}

$$
\operatorname{EV}\left(T^{*}, x^{*}, j, z\right)=\sum_{x^{*}} \sum_{T^{*}} p\left(x^{*} \mid T^{*}\right) p\left(T^{*} \mid z\right) \bar{V}\left(T^{*}, x^{*} j, z\right)
$$

Expected payoff for DM Type $j=\operatorname{sum}$ of expected payoffs for each potential outcome $\left(T, x^{*}\right)$ for choice $z$. $T^{*}$ is the number of DMs expected to Take, and $x^{*}$ is the state of the commons, assumed in a particular outcome of the decision trecs. $V\left(T_{i}, x_{i}, j, z\right)$ is Eq. (6.14).

\section{- Genetic algorithm}

\section{Fitness}

$$
F_{i j}=R V_{i j}=\bar{V}\left(T_{i}, x_{i}, j, z\right)
$$

Fitness of genotype $j$ at iteration $i=$ realized value $R V_{i j}$ which is calculated as $V_{i j}$ (Eq. 6.14).

Genotype fractions

$$
\operatorname{Pr}(j, i+1)=f\left[\operatorname{TSProb},\left(F_{i j} / \frac{1}{T_{\max }} \sum_{j} F_{i j}\right]\right.
$$

Probability of genotype $j$ being a progenitor genotype to the population of DMs in iteration $i+l$ is a function of the parameter TSProb and the payoff received by genotype $j$, compared to the average payoff received by the effective population of potential Takers, $T_{\operatorname{mar}}$, in iteration $i$. 


\section{CONCLUSION}

A computer model of the Tragedy of the Commons will be most useful if it simplifies the complexities of this social dilemma enough to help think it through more effectively, without eliminating or fatally altering aspects that are critical to the definition of, or solution to, the problem. One of the major challenges of this work is that, to be valid, such a model has to integrate knowledge and understanding from the fields of biology, evolution, ecology, psychology, political science, and economics (not to mention computer science), and it must do so in a way that is acceptable and comprehensible to people in all these fields.

Because this model relies on well-established methodologies from each of these fields, the methods for each component of the model should be straightforward to evaluate. The DMs use particularly simple versions of the workhorse of prescriptive decision making, expected multiple attribute value (EMAV) decision trees. The commons model is an uncomplicated, uniform ecological system with an ordinary logistic regeneration function, a simple stochastic disturbance generator, and single species whose value to humans is represented by simple continuous functions. The process of social change is simulated with a GA that is relatively unsophisticated compared to other more complex GAs being used by artificial life researchers today (Riolo, 1992).

It is, of course, not just the components, but the linkages, that are important. 
The feedback loop that links the commons with the DMs provides the mechanism that ties these components together. The idea that the actions of human decision makers (represented by the choices of the DM genotypes) may have effects on a public resource (represented by the change of state of the commons in response to the DM actions), and that the state of the common resource may in turn have effects on the human decision makers (via the payoff they receive as a result of their actions), is hardly novel. What is novel is including these linkages in a model in which the linkages are not only relatively simple, but more important, reasonably believable as well. 


\section{Chapter VII}

\section{MODEL DEVELOPMENT}

This study was conducted in three phases. In the first phase, the computer simulation was developed and used to simulate Garrett Hardin's formulation of the TOC. The objective of this phase was to develop and validate the simulation by applying it to a case where there is broad agreement as to what the results should be.

In the second phase, an analysis of the simulation parameters was performed via a series of factorial experiments. The objectives of this phase were to refine the model and to determine the relative impacts of the model parameters. These first two phases, which are discussed in this chapter, were somewhat iterative because the basic formulation of the model and the computer code were revised as more was learned from initial experiments.

In the third phase (discussed in the next chapter), potential solutions to the TOC were further investigated, by running simulations and by analyzing the decision models using game theory. The objective of this phase was to investigate the answer to two questions: "Under what conditions could the TOC be solved?" and, "Was Hardin right?" 


\section{PHASE I: MODEL DE.VELOPMENT}

\section{Hardin's TOC}

Hardin described two situations for his villagers. In the first, historical, phase, population growth was held in check by factors such as disease and war. Villagers harvested as much as they wanted from the commons, and because the equilibrium population was relatively low, their harvest rate remained below a sustainable equilibrium value. In the second, modern, phase, the "happy day" came when disease and war no longer checked population growth, and harvest levels grew to exceed the commons capacity for regeneration. Eventually the villagers destroyed the commons (Hardin, 1968).

In Hardin's TOC, the community was populated only by herdsmen: there were no Refrainers, only Takers. Hardin had no Refrainers in his TOC because, as he argued, the "remorseless logic" of the commons dictates that DMs have no choice but to Take: Refrainers receive no payoff, since the only payoff Hardin considered was from Taking. Because it provides no payoff, Refraining would not be what Axelrod calls an Evolutionarily Stable Strategy (Axelrod, 1984) and thus would be evolutionarily self-eliminating.

To simulate Hardin's historical situation, the population growth rate would be zero. The commons regeneration rate need only be greater than or equal to the number 
of potential Takers. The population size is thus determined by the setting for the regeneration rate constant ${ }_{r_{o}}$ (or vice-yersa). For this case, the simulation would be expected to produce a long-term equilibrium where all DMs choose to Take and the commons hovers around some constant non-zero leval.

To simulate Hardin's modern situation, the only change is that the population growth rate would be greater than zero. For this case, the number of Takers would be expected to grow to exceed the commons ability to regenerate, and the commons to then be destroyed.

These assumptions about Hardin's two village situations were used to determine $r_{0}$ and the population growth rate $G$. The maximum number of potential Takers, and thus the equilibrium harvest level for Hardin's historical situation, was set to 100 in order to leave plenty of computer memory for growing populations, while still allowing a large enough population of DMs so the GA would work properly. It was assumed that the equilibrium harvest level would equal the maximum sustainable yield $M S Y$. Recall that the (non-stochastic) regeneration rate equation is (Grant, 1986, 248):

$$
r(x)=r_{0} x-r_{0} x^{2} / K
$$

where $r(x)$ is the regeneration rate of the commons, $x$ is the state of the commons, $r_{0}$ is the regeneration rate constant, and $K^{7}$ is the maximum possible state of the commons

\footnotetext{
${ }^{7} \mathrm{~K}$ is ordinarily used in biology to indicate carrying capacity, which is what $x_{\max }$ means here. "Carrying capacity" means the number of commons units that the commons can support, rather than the number of lumans the commons can support. It would be "cleaner" to dispense with $K$ and simply use $x_{\max }$, but the Grant equation is such a fundamental of biology that it was preferred to use the usual form.
} 
$x_{\max } . M S Y$ is assumed to be the maximum possible regeneration rate, which occurs at $K / 2$ for Grant's Equation. Thus, for $x=K / 2$ and $r(x)=100, r_{0}$ is 0.04 .

The regeneration rate of a real commons is never as deterministic as Eq. (7.1) implies, however, and so stochasticity is added, in a simple way, by sampling from a normal distribution with a mean of $r(x)$ and some desired standard deviation. In fisheries populations the actual regeneration rate often varies within a range that is at least \pm two or three times the nominal rate (Huntington, 1994). Assuming that this approximation represents \pm one standard deviation, then the distribution for the actual regeneration rate would be normally distributed with a mean of $r(x)$ and a standard deviation of $2 r(x)$ or $3 r(x)$.

As for the profit function, all that is really necessary for Hardin's TOC simulations is that profit be greater than zero, since the payoff for Refraining is zero. However, it was argued previously that a more realistic representation than Hardin's would have profit as a moderately steep function of supply. Thus, a moderately steep slope (Gwartney and Stroup, 1980) of -1.5 was chosen initially. The result for the situation where the population of the village is constant, and all choose to Take, is shown in Figure 7.1.

For the "modern" situation where population is no longer held in check by disease and war, the ordinary exponential population growth equation (Eq. (6.5)) is used to simulate population growth. The population growth equation is in terms of the time increment $\Delta t$ and the time increment in the simulation is defined only as the time 
between decision iterations. A reasonable assumption would be that this time increment would be on the order of weeks or months - probably not days or years. The exact value is not very important, because any growth rate greater than zero should produce Hardin's expected result. Human population growth is currently $1.5 \%$ per year, worldwide (Brown, Lenssen, and Kane, 1995), so as an approximation (based on the decision increment being about one tenth of a year), a growth rate of about one tenth of the current annual rate was used.

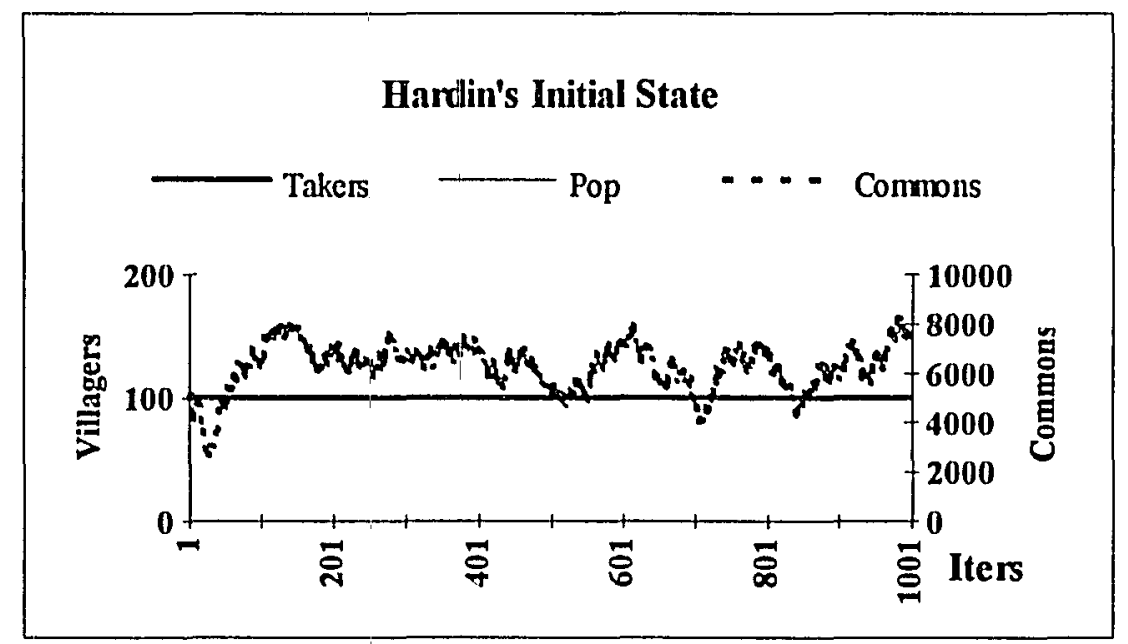

Figure 7.1. Hardin's TOC where the population is held constant, DMs do not take "Conscience" into account, and the commons is moderately stochastic (coefficient of variation $=2.0$ ). This simulation ran 1001 iterations lters. Because the entire population of potential Takers chooses to Take, the two lines for Pop and Takers are indistinguishable from each other.

The result is shown in Figure 7.2: as Hardin predicted, harvest levels that were sustainable for limited population prove inadequate to satisfy a growing population, and as a result the commons is destroyed.

Regardless of which of the three possibilities is used for how the profit function 
varies with growing populations (discussed in the "Decision Model" section of the previous chapter), Figure 7.2 is the result. This is because there is no payoff for Refraining, only for Taking. Similarly, assuming declining marginal value for the profit function also has no effect, as long as there is no payoff for Refraining.

\section{"Conscience"}

Although Hardin made no allowance for conscience, the "Conscience" function is an important element in this work because something like conscience is an important component of some of the proposed solutions. "Conscience" needed to be included in the experiments of the next phase, and so preliminary values for the slope and $y$ intercept for the "Conscience" function were determined in this first phase.

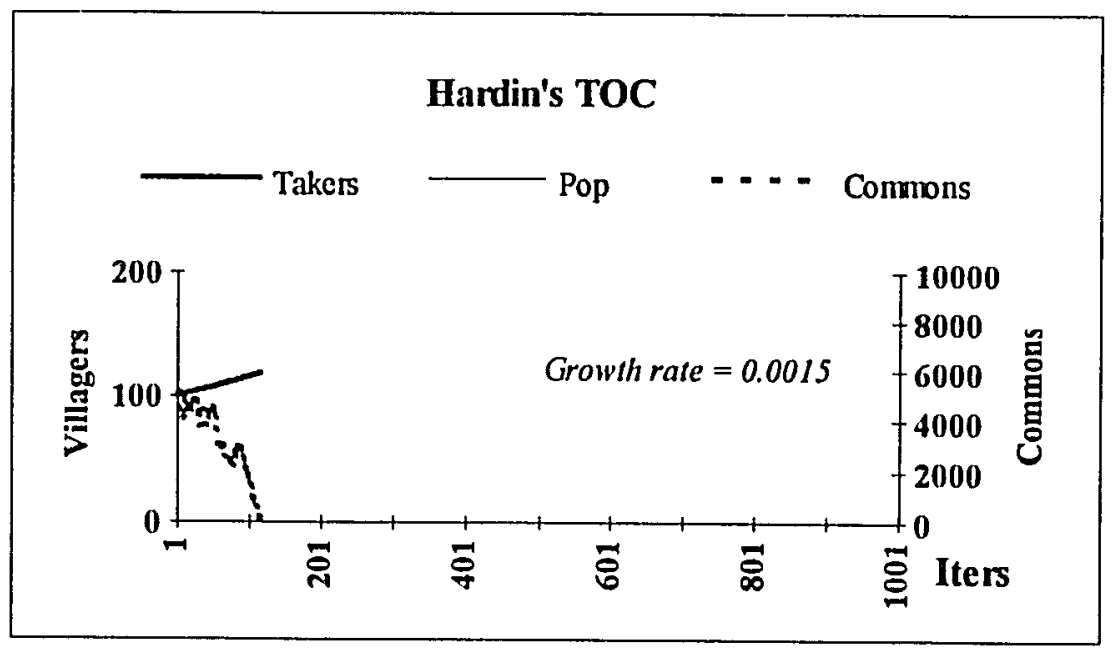

Figure 7.2. Hardin's TOC where the population, made up only of Type I DMS, grows at $G=0.0015$ per time increment. DMs do not take "Conscience" into account, so the number of Takers is the same as the population of potential Takers, and the commons is moderately stochastic (coefficient of variation = 2.0). The entire population chooses to Take, and so the lines for Pop and Takers are indistinguishable from each other. 
The slope and $y$-intercept were determined by assuming two things:

1. "Conscience" would produce a solution for a population that is twice the sustainable population without "Conscience": since the sustainable population for Hardin's historical equilibrium case is 100 , "Conscience" is required to offset profit enough that a population of $200 \mathrm{DMs}$ would only harvest 100 or fewer units of cómmons, on average.

2. "Conscience" would cause some DMs to switch to Refraining once the commons dropped below $K / 2$. This requirement means that some of the DMs, such as Types I and II, could always Take, but that others, such as Types III and IV, would Refrain; and that there would be enqugh Refrainers to keep the harvest level below the regeneration rate. Stochasticity is assumed to be zero, initially.

The Type III DMs were used for this calculation. The aggregate value of Taking was set equal to the value of Refraining, for $x=K / 2=5000$, and the number of Takers was set to the sustainable number, 100:

$$
\begin{aligned}
& \left.\bar{V}_{l I I}(\text { Take })=w_{\pi} \pi(T)-C(x)=\bar{V}_{l l l}(\text { Refrain })=C(x) \quad \text { (from Eq. }(6.17)\right) \\
& \pi(100)=2 C(x) \\
& k\left(T_{\max }-T\right)=2 m(K-x) \quad \text { (from Eq. (6.8) and (6.12)) } \\
& 3.0(200-100)=2 m(5000)
\end{aligned}
$$

For a profit function $\pi(I)$ with a moderately steep slope $k$ of 3.0 , the slope $m$ for $C(x)$ was found to be 0.03 . 
The exact values used for these assumptions are not very important, because the question is not what the slope has to be, but rather, once a slope is identified that meets the two criteria, can it be made to work for growing populations? All that is needed is a "Conscience" function that increases as the commons declines, that impacts some of the DMs (e.g., at least the Type III's and IV's) some of the time, and causes enough of them to Refrain so that the commons can survive at twice the initial sustainable population (Hardin's historical equilibrium situation). If a particular $m$ proved inadequate to solve the TOC for twice the initial sustainable population, it could be increased. The impacts of different values for this slope were examined with the factorial experiments of Phase II.

The results of including "Conscience", for a population that grows only to twice the initial sustainable population, are shown in Figure 7.3 (compare to Figure 7.2, where the simulation parameters were the same but none of the DMs had a "Conscience").

The difference between Figure 7.2 and Figure 7.3 is that including "Conscience" for Type II, III, and IV DMs is enough to cause the villagers to harvest sustainably, as long as the population of potential fishers remains below twice the original sustainable size (or $2 * 100)$. Unfortunately, if the population of potential fishers is allowed to grow beyond 200 , eventually the temptation of increased profits (due to increased demand) outweighs the costs of "Conscience" for enough of the villagers that they still end up destroying the commons, as shown in Figure 7.4 - even though less than half 
of them are Taking. The simulation in Figure 7.4 is the same as that in Figure 7.3, except that there is no limit on population growth.

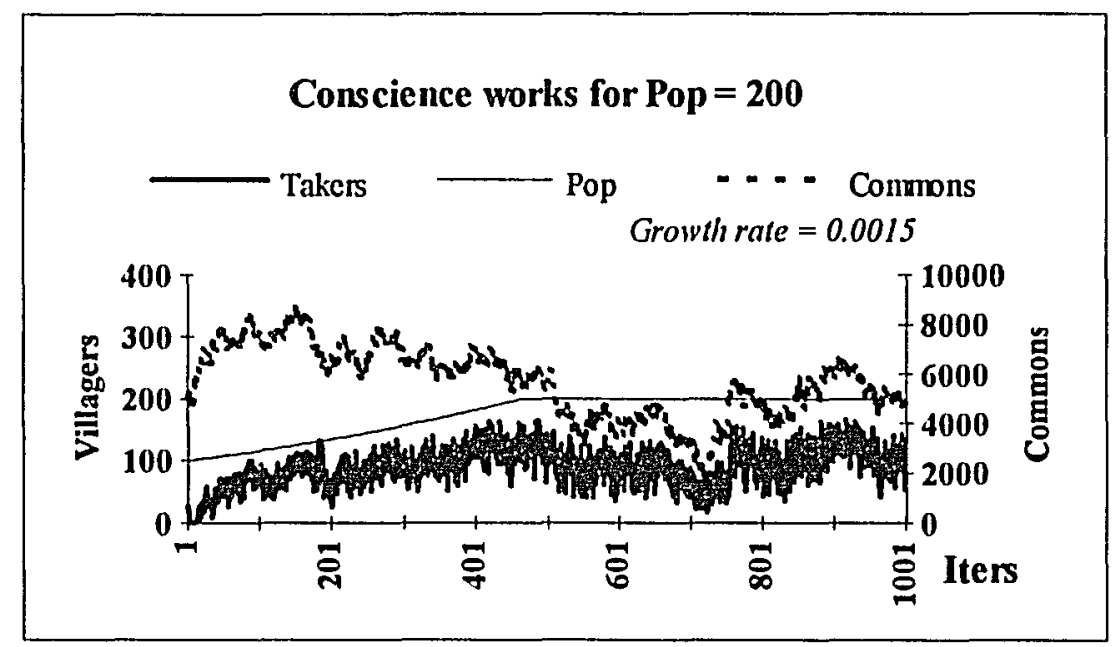

Figure 7.3. "Conscience" prevents the destruction of the commons, for a moderate, maximum population of potential fishers of twice the initial sustainable size (Figure 7.1). Stochasticity is moderate: the coefficient of variation $\mathbf{= 2 . 0}$.

The solution fails if the population of potential fishers exceeds 200 (twice the historical sustainable population) because the "Conscience" function parameter $\boldsymbol{m}$ was derived with an arbitrary assumption that "Conscience" would work up to about double the initial population. The "Conscience" function can be given a steeper slope such as $m$ $=0.06$, so that the village will harvest sustainably at a potential fisher population of, say, 300, but then as the population grows beyond that, the solution will fail again (see the solution simulations in Chapter VIII).

Solutions based on this simple "Conscience" function may be found to work for any maximum population size, but as long as demand increases with increasing 
population, and "Conscience" increases as the state of the commons declines, eventually these solutions all must fail. Hardin (1968) said that conscience could not

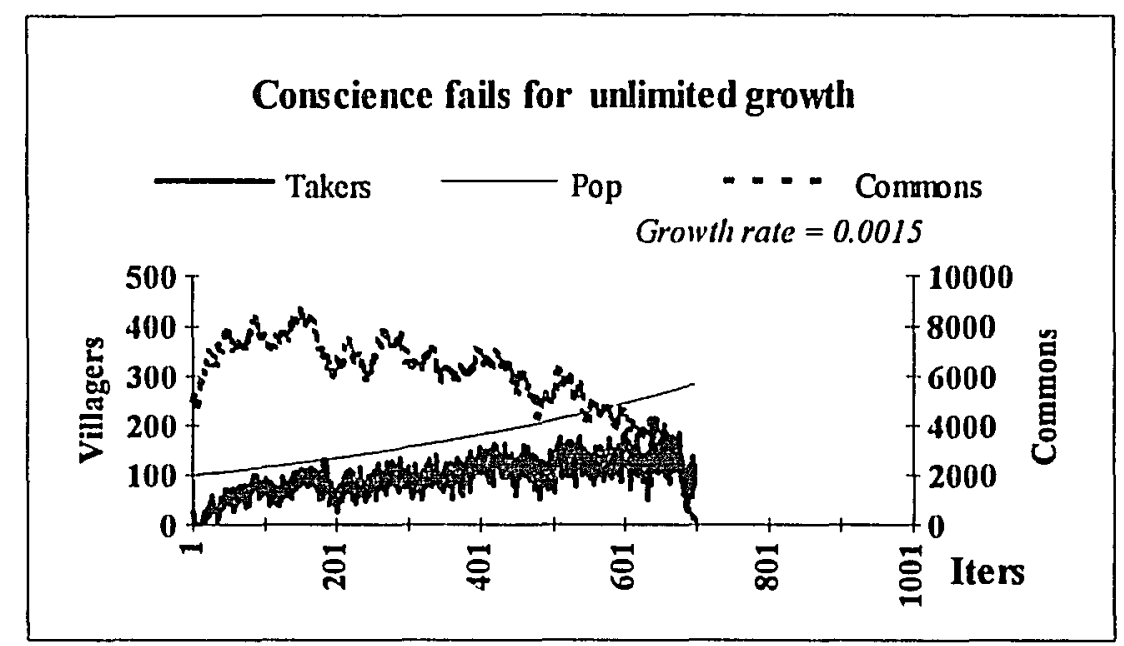

Figure 7.4. "Conscience" solution fails for unlimited population growth. Stochasticity is moderate: the coefficient of variation $=2.0$.

solve the TOC, mainly because people would feel like fools if they Refrained so that others could Take. He did not allow for the possibility that there might be some DMs whose "Consciences" never permitted them to Take, as there are here; nor even that "Consciences"might work up to a point, as it did here. Nonetheless, he did say that no voluntary solutions could work over the long run if population were allowed to grow. In this first phase of this work, under the simplest of assumptions, his argument is supported, and the simulation performs consistently with Hardin's predictions. 


\section{PHASE II: FACTORIAL EXPERIMENTS}

The objectives of the second phase of the study were to further refine the simulation and to determine the reiative impacts of the model parameters. It is important to emphasize that these experiments were conducted only to gain a general understanding of how the parameters in the simulation interact, which simulation parameters are the most important, and what the most useful settings might be for the solution simulations that follow. These experiments were not as rigorous, therefore, as they might have been if the dissertation were primarily an empirical, rather than a theoretical, investigation: in an empirical dissertation, the data would have to be rigorously analyzed in order to confirm or disconfirm a particular hypothesis. In this theoretical investigation, the factorial experiments are used as a convenient way to help develop a theoretical model.

This approach is based on Lenore Fahrig's (1991) work using factorial experimental methods to analyze population dynamics in stochastic ecological sinulations. Fahrig used factorial experiments to make the sensitivity analysis for multiple variables more efficient. She had to use statistical analysis methods because the simulations she was interested in were stochastic. The work here is also stochastic because GAs are not deterministic, and thus the factorial sensitivity studies also have to be analyzed statistically. Each simulation run is a sample from the effectively infinite number of simulations that could be run, because the stochastic elements of the 
simulation are determined by the random number generator, which is initialized with a random number seed set by the user.

Fahrig argued that a major problem with simulations in general, and stochastic simulations in particular, is that the outcomes of such simulations often depend on the particular parameter values chosen. She pointed out that it is crucial to the validity of any simulation to formulate relationships between the parameters chosen and the output. In order to demonstrate the method she was advocating, she used factorial experiments to analyze some existing stochastic simulations of ecosystem population dynamics, and then used statistical analysis to determine the functional relationships between the parameter ranges and the output.

In this work, all the major components are essentially created from scratch because there are no empirical data that can be directly used. For example, the regeneration function of the commons is loosely based on a fisheries regeneration model. Its stochasticity is modeled with a normal probability distribution (see Chapter VI, Section 2). The DMs' decision models are based on a distillation from a very broad review of the literature on decision making and economics, but the assumptions used for the profit and "Conscience" functions are simplistic (see Chapter VI, Section 5). The mutation rate and reproduction control parameters of the $\mathrm{G} A$ are arbitrary (see Chapter VI, Section 3). Since the particular values used for these important parameters determine the results, it is important to be able to characterize the output as a function of the input ranges selected. Only in this way can the results be extended to wider 
applicability than to just this particular model.

The variables in the model which were examined with the factorial experiments are: the range of alleles included for each of the genes representing the DMs' beliefs and values, the parameters in the profit and "Conscience" functions, the stochasticity of the commons, and the mutation rate and reproduction control parameters of the GA.

A series of four factorial experiments and numerous trial-and-error explorations were conducted during the model and computer code development work. Results of these were used to refine the computer code and the model, to determine the appropriate levels for experimental parameters, and to determine what the response variables should be. The fifth factorial experiment was performed on the final model, and is a synthesis of all the earlier explorations. This last experiment is the only one that is discussed in detail here.

\section{Methods}

The preliminary four experiments indicated that the variables that might be important were: the slopes of the profit and "Conscience" functions (variables $k$ and $m$, respectively), stochasticity of the commons Stoch, mutation rate $m r$ of the GA, and the probability TSProb that relatively higher-fitness genotypes from one iteration would be subjected to mutation and recombination and thus become "parents" or progenitors ${ }^{8}$ to

\footnotetext{
8 "Parents" implies biological reproduction, and because this is a model of social evolution, not biological evolution, another word was needed that did not seem so closely tied to the biological notions of parents and children.
} 
the next iteration.

The probability tables (Table IV) used in the decision trees were not found to be significant by any of the four preliminary experiments $(p>0.2)$, even when they were varied to an unreasonable extent (e.g., optimists thought there was only a $10 \%$ probability that the commons would be in Good condition if most DMs Refrained, instead of the nominal $80 \%$ shown in Table IV). This finding was a surprise, because it seemed reasonable that people's choices might be sensitive to how certain they were about what others would do and what the state of the commons might be. The reason the estimates turned out to be insignificant is that the decision trees were set up so that the Type I and IV DMs both have dominant strategies (Type I's always Take and Type IV's always Refrain), Type II's almost always Take, and only Type III's change their minds when the commons becomes degraded. Probability estimates are thus only really important to Type III's, who did not have enough of an impact on the factorial experinnents to indicate statistical significance for the probability estimates. The simulations were sensitive to probability estimates insofar as the GA selected the optimism or pessimism allele for gene $g_{0}$ when some of the solution mechansims were simulated (see the "Solution Simulations" section of Chapter VIII), but this effect was not detected by the factorial experiments.

All of these experiments were run with a constant population size of 200 , which was the "cutoff" value used to define the "Conscience" function (the default "Conscience" function can preserve the commons up to a population of about 200). In 
the preliminary experiments, the commons state was initialized at $x_{0}=K * 0.25, K * 0.5$, or $K * 0.75$ (recall that $K$ is the maximum $x$ can be). There was no significant difference in results for $x_{0}$ of $K * 0.5$ and $K * 0.75$, but the commons tended to quickly go extinct if it was initialized at $K * 0.25$. It was decided to assume that the simulation began at the equilibrium point assumed for Hardin's sustainable population, which was $x_{\infty} \approx K$ * 0.5. The settings used in the fifth factorial are shown in Table V.

Earlier experiments were replicated three to five times, and acceptable (defined below) models were easily achieved with three replications. With two levels for each of five variables, and three replications, the number of runs was thus 96 . All other assignable parameters required in the computer code were held constant for all 96 runs, except for the random number seed. The random number generator is what drives all the stochastic components of the simulations. The random number generator is used by the GA to create the initial genotypes, and to simulate the stochastic processes of progenitor selection, mutation, and recombination (see Chapter VI, Section 3). It is also used in the commons model to simulate stochastic variation in the regeneration rate via the normal distribution sampling function (see Chapter VI, Section 2). The sequence of numbers generated by the random number generator is determined by the random number seed. In the first simulation run for any of the experiments, the seed was selected from a random number table in a statistics text. For each run thereafter, the random number generator printed out a random number at the end of each trial, and this was used as a seed for the next trial. 


\begin{tabular}{|l|c|c|}
\hline Parameter & Low level & High level \\
\hline Slope of profit function, $k$ & 1.5 & 3.0 \\
\hline Slope of "Conscience" function, $m$ & 0.02 & 0.04 \\
\hline Stochasticity, Stoch & 0 & 2.0 \\
\hline Mutation rate, $m r$ & 0.001 & 0.01 \\
\hline $\begin{array}{l}\text { Probability of selection for } \\
\text { mutation and recombination, } \\
T S P r o b\end{array}$ & 0.5 & 0.9 \\
\hline Initial state of the commons, $x_{0}$ & 5000 & 5000 \\
\hline Population (potential fishers) & 200 & 200 \\
\hline Commons regeneration rate, $r_{0}$ & 0.04 & 0.04 \\
\hline
\end{tabular}

Table V. Seltings for fifth factorial experiment.

Although a variety of response variables (average state of the commons over time, average number of Takers, pattern of long-term behavior, and iterations to extinction) was recorded in the earlier experiments, by the fifth experiment it was clear that iterations to extinction Iter was the only one that provided consistently useful ANOVAs. The simulations were run up to 1800 iterations, because in earlier experiments extinction never occurred beyond about 1200 iterations and otherwise, stable patterns were established by the 500 th or 600 th iteration. For the statistical analysis, the trials that exceeded 1800 iterations were coded as 3600 , in order to ensure that the difference between trials that led to extinction and trials that did not were adequately differentiated. Significantly different results, particularly $R_{a d j}^{2}$ and mean square error MSE, were not achieved by increasing 3600 to 7200 , and the ANOVAs for the 3600 codings produced no outliers nor high leverage cases. Results became ambiguous for codings below about 2500 . 
Regressions and ANOVAs were conducted using Systat ${ }^{\circledR}$ (1992) The objectives for the model development were:

- Reasonableness: find a model that makes intuitive sense, i.e., one for which it is possible to explain why some factors are included and others are not.

- Explanatory power: find the minimum number of variables that explain the maximum amount of variation in the data, as indicated by $p<0.015, R_{a d j}^{2}$, and mean square error MSE.

- Validity: find a model that is statistically defensible, as indicated by plots of the residuals versus the regression estimates, normal probability plots of the residuals, and absence of high leverage or outlier cases.

The models were developed by performing regressions and ANOVAs on all variables and all two- and three-factor interactions, and then manually adding or deleting variables, including interactions; as well as by having Systat ${ }^{\circledR}$ perform backwards and forwards stepping.

\section{ANOVA: Iterations to Extinction}

The regression and ANOVA for the model that fits the objectives the best, for the response variable "iterations to extinction" Iters, are shown in Table VI.

Table VI shows a model in which all five factors are statistically significant, as main effects or in interactions. It is important to note that the regression is not intended to be used to predict continuous system response for settings between the two 
levels of each paranneter, but rather as a relatively easy way to conduct a sensitivity analysis. Regression is a convenient way to evaluate the relative importance and direction of effect of several parameters at once, by performing $t$-tests on the main effects and lower level interactions.

These experiments were not conducted in order to establish predictive relationships, but rather to evaluate the relative impacts of parameters that drive a

\begin{tabular}{|c|c|c|c|c|c|}
\hline$R_{a d j}^{2}=0.812$ & Standard error & $=628.4$ & \multicolumn{3}{|c|}{ Dependent variable $=$ Iters } \\
\hline Variable & $\hat{b}$ & $\begin{array}{l}\text { Standard } \\
\text { error }\end{array}$ & $\begin{array}{l}\hat{b} \\
\text { standardized }\end{array}$ & $t$ & $p$ (2-tail) \\
\hline Constant & -899.1 & 624.9 & 0 & -1.4 & 0.154 \\
\hline Stochasticity $S$ & -748.9 & 242.5 & -0.5 & -3.1 & 0.003 \\
\hline $\begin{array}{l}\text { Mutation rate } \\
\text { mrr }\end{array}$ & 39581.4 & 4891.0 & 1.4 & 8.1 & 0.000 \\
\hline Profit slope $k$ & 6.76 .2 & 208.7 & -0.4 & 3.2 & 0.002 \\
\hline $\begin{array}{l}\text { Selection } \\
\text { Probability } \\
\text { TSProb }\end{array}$ & $\begin{array}{l}-2699.0 \\
\mid\end{array}$ & 618.5 & -0.4 & -4.4 & 0.000 \\
\hline S*TSProb & 1012.3 & 320.7 & 0.5 & 3.2 & 0.002 \\
\hline$S * \|$ & -3326.2 & i295.7 & -0.2 & -2.6 & 0.012 \\
\hline TSProbtiur & -39791.7 & 6478.3 & -1.0 & -6.1 & 0.000 \\
\hline TSProb*m & 268003.9 & 22216.4 & 1.8 & 12.1 & 0.000 \\
\hline TSProb*k:*m & -75764.7 & 9064.5 & -1.4 & -8.4 & 0.000 \\
\hline
\end{tabular}

ANALYSIS OF VARIANCE

\begin{tabular}{|l|l|l|l|l|l|}
\hline Source & $\begin{array}{l}\text { Sum of } \\
\text { squares }\end{array}$ & $\begin{array}{l}\text { Degrees of } \\
\text { freedom }\end{array}$ & mean-square & F-ratio & $p$ \\
\hline Regression & $0.1657 \mathrm{E}+09$ & 9 & $0.1841 \mathrm{E}+08$ & 46.6 & 0.000 \\
\hline Residual & $0.3396 \mathrm{E}+08$ & 86 & $0.3949 \mathrm{E}+06$ & & \\
\hline
\end{tabular}

Table VI. Regression and ANOVA for the response variable indicating number of iterations to extinction Iters.

simulation model and to get an idea of what the range of settings ought to be for the solution simulations in Phase III. If the regression were to be used to develop predictive relationships, it would imply that the regression function that relates each of 
the independent variables with the dependent variable is monotonic: i.e., that if the dependent variable were varied continuously from the low to the high level, then the response would also either increase or decrease continuously, with no hump or valley in between. In a rigorous exploration, if there were any doubts, it would be advisable to conduct at least a threc-level factorial in order to be able to look at the response surfaces and to check for non-monotonicity. This exploration is simply a convenient way to conduct a rough sensitivity analysis as part of model development, and it would thus be hard to justify going to such extremes.

The single factor with the most important impact on how long the commons survives is the mutation rate $m r$ (standardized beta $\hat{b}=1.4, p<0.0005$ ). Since $\hat{b}$ is positive, it indicates that Iters increases with $m r$ : the commons lasts longer when $m r$ is at its higher level. The mutation rate is important because it constantly (re)introduces variety into the evolutionary model by randomly, with some small probability $\mathrm{mr}$, switching alleles at a gene locus (only one mutation is allowed per genome, per iteration). A mutation means that a DM genome could be changed from an optimist to a pessimist, or from a Type I who will always Take to, say, a Type IV who will always Refrain.

There are only three, dichotomous genes in this GA: $g_{0}$, the optimism/pessimism gene; $g_{l}$, the gene that determines the coefficient for the "Conscience" penalty $C(x)$ for Taking, and $g_{2}$, the gene that determines the coefficient for the "Conscience" reward for Refraining. Since three dichotomous genes means 
there are only eight $\left(2^{3}\right)$ genotypes possible, the simulation tends to converge quickly. When it does, there is no variety in the population to allow the DMs to adapt to changing conditions. For example, if the simulation converges quickly and consists of all Type I's, they always Take, even when the commons is almost completely gone. There are then no Type III's or IV's left who could become more prevalent with the changing conditions, thus perhaps preventing the over-harvesting soon enough to avoid destroying the commons.

One pararneter that helps prevent premature convergence is $m r$; TSProb is the other (standardized $\hat{b}=-0.4, p<0.0005$ ). TSProb is the probability that higher-fitness genotypes in one iteration will be selected as progenitors for the next iteration (see Chapter $\mathrm{V}$, Section 3 ). The standardized regression coefficient $\hat{b}$ is negative for TSProb, indicating that the commons survives longer when TSProb is lower.

If TSProb is relatively low, then a relatively high proportion of less fit genotypes persists in the population. The variety that is maintained in the population by high $m r$ and low TSProb is important for allowing the villagers to respond to changing conditions. TSProb and $m r$ preserve a healthy contingent of "crackpots" in the population: DMs who persist in doing the "irrational" thing at one time often turn out to be "rational" later. The fitness function acts as enough of a gatekeeper to prevent too many "crackpots" from taking over the population.

Figures 7.5, 7.6, and 7.7 illustrate typical simulation response patterns for the two diversity parameters. In these three figures, the other experimental variables are 
held constant (profit function parameter $k$, "Conscience" function parameter $m$, and stochasticity Stoch — discussed below — are all low; effective population size $T_{\max }$ is held at 200 , which is twice the sustainable size). In Figure 7.5, the mutation rate is low and the progenitor diversity is also low; the commons quickly crashes at 172 iterations. Although the DMs do alter their behavior as the commons declines, there is not enough diversity for an adequate number of Refrainers to take over in time to prevent the commons crash.

In Figure 7.6, the only difference is that the progenitor diversity is high (i.e., TSProb is low); the commons lasts longer (486 iterations), but still crashes. This indicates that lowering TSProb in order to maintain progenitor diversity helps, but not enough. The simulation exhibits fairly erratic behavior because the behavior of the DMs is more erratic: with TSProb only at 0.5 , approximately half the progenitors selected for each new iteration are lower-scoring genotypes. The GA is doing an inefficient job of "optimizing" each new iteration, which is why there is more diversity; but it is also why the behavior tends to be more erratic. It is not unusual, in fact, for simulations with high TSProb settings to exhibit patterns that look like undamped oscillations.

In Figure 7.7, both diversity parameters support high diversity and the commons lasts forever. Note too that the erratic behavior typical of high progenitor diversity and low mutation rate has been replaced with a fairly smooth, steady-state oscillation. This is a typical pattern when both diversity parameters are high: apparently the higher mutation rate acts as a damper to counteract the wilder swings that high 
TSProb setting causes.

\section{Low progenitor diversity and mutation rate}

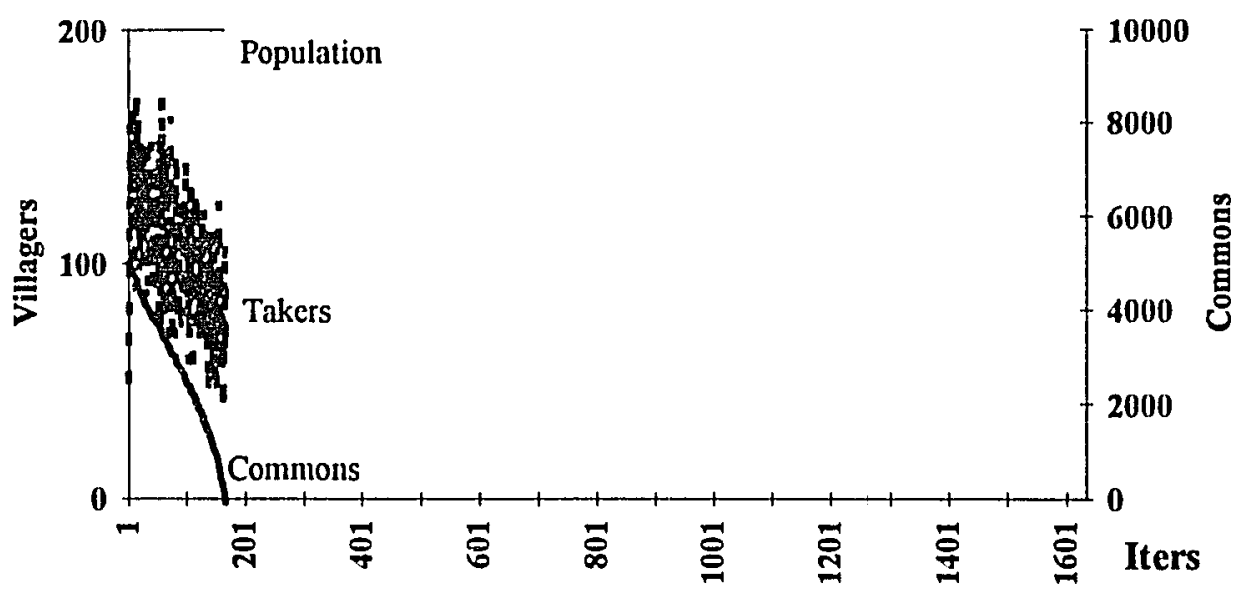

Figure 7.5. Simulation with low population diversity on both diversity parameters: TSProb is 0.9 (low progenitor diversity) and $m r$ is 0.001 (low mutation rate).

\section{High progenitor diversity but low mutation rate}

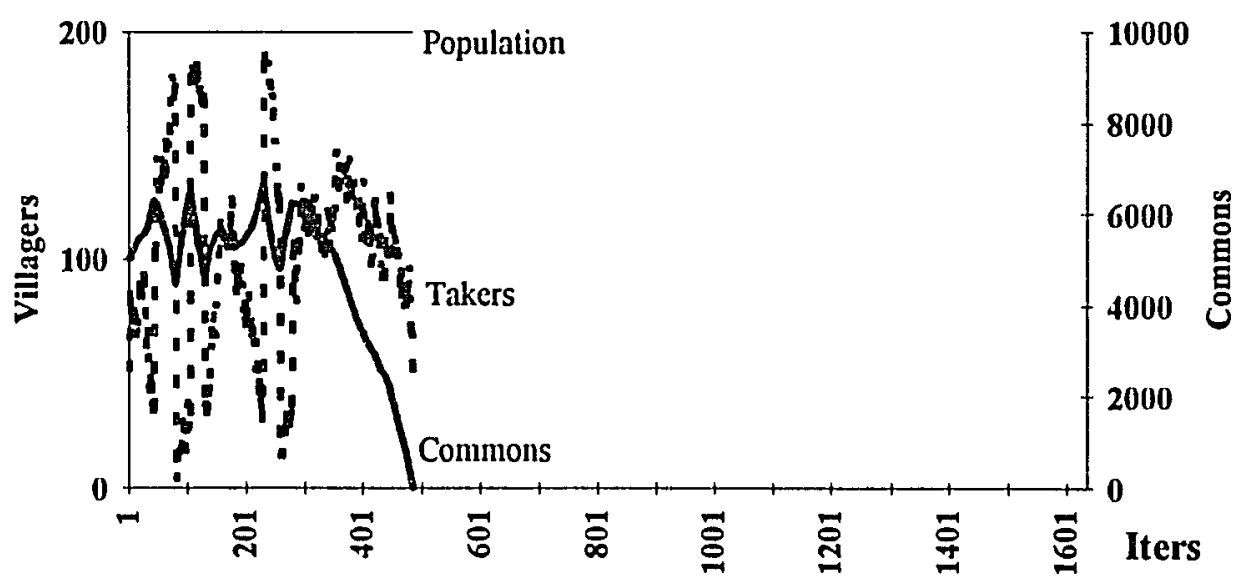

Figure 7.6. Simulation vrith high progenitor diversity $(T S P r o b=0.5)$ but low mutation rate $(m r=0.1)$. 
High progenitor diversity and high mutation rate

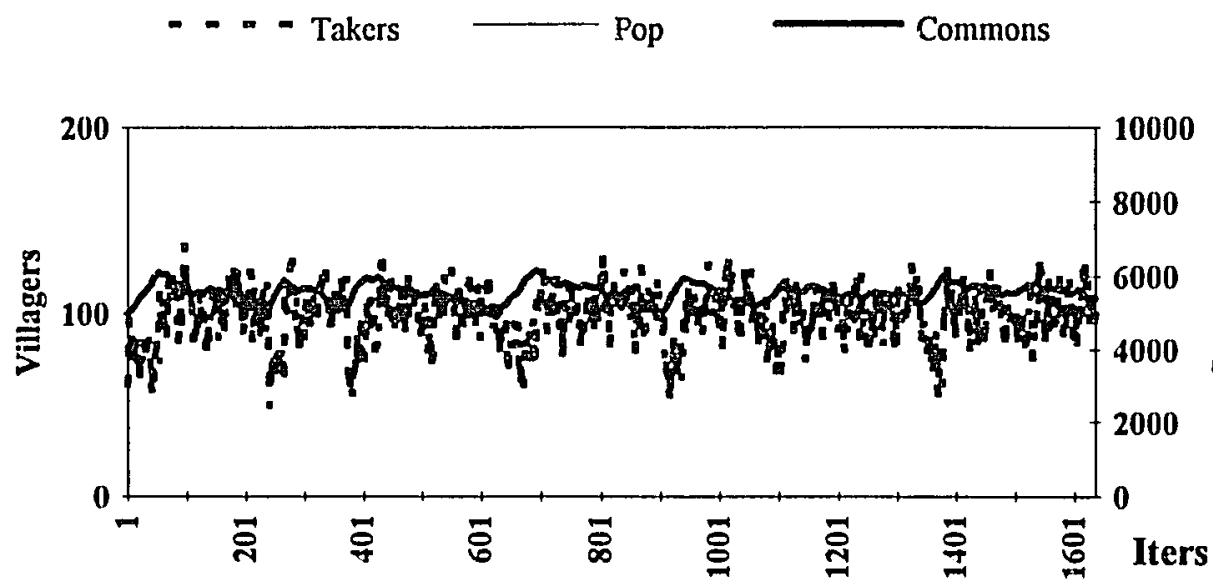

Figure 7.7. Simulation with high population diversity on both diversity parameters: TSProb is 0.5 (high progenitor diversity) and $m r$ is 0.1 (high mutation rate).

Commons stochasticity is also important as a main effect (standardized $\hat{b}=$ $-0.5, p=0.003$ ) because the more erratic the commons regeneration rate, the more difficult it is for the DMs to respond appropriately. In other words, the negative $\hat{b}$ indicates that as stochasticity increases, the number of iterations the commons persists decreases. For example, Figure 7.8 shows the same simulation as Figure 7.7 except that stochasticity is high in Figure 7.8 whereas it is zero in Figure 7.7.

In Figure 7.8 the commons crashes, even though the number of Takers hovers around the sustainable number (100). This is an example of why the traditional fisheries harvest management method of maintaining harvest levels at a theoretical maximum 
sustainable yield (MSY) is sometimes criticized (Holling, 1978; Ludwig, Hilborn, and Walters, 1993): MSY management is based on an assumption that the regeneration rate of the commons can be reliably estimated. When stochasticity is high, however, more margin needs to be built into the harvesting level, because there is some unknown probability that the true MSY is considerably less than the theoretical one at any time.

In Figure 7.8, MSY is theoretically 100, but the stochasticity of the commons regeneration rate makes the real MSY something less than the assumed MSY.

Overharvesting results, and the commons is not able to recover.

\section{High diversity, high stochasticity}

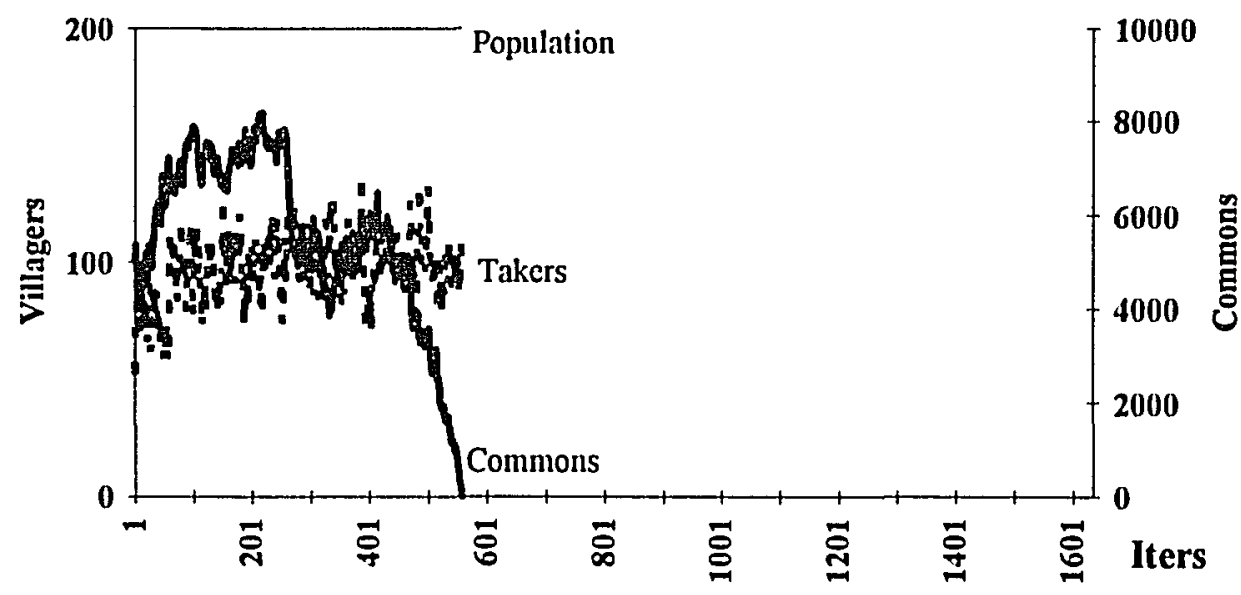

Figure 7.8. Simulation with high population diversity on both diversity parameters: TSProb is 0.5 (high progenitor diversity) and $m r$ is 0.1 ?high mutation rate) (the same as the simulation in Figure 7.7), but with high stochasticity (Stoch $=2.0$ )

The profit function parameter $k$ is the last important main effect (standardized

$\hat{b}=0.4, p=0.002$ ) because $k$ determines the slope and $y$-intercept of the profit 
function. $\hat{b}$ is negative, meaning that extinction happens faster with greater $k$. This is because increasing $k$ increases the slope and magnitude of the profit function (i.e., increases demand) and thus, profit becomes more tempting ( $k$ is discussed at length, and plots of simulations illustrating the impacts of $k$ are shown, in the solutions discussion of Chapter VIII).

The interaction plots illustrate the implications of interactions among parameters. For example, the interaction Stoch $* m r$ (standardized $\hat{b}=-0.2, p=0.012$ ) is shown in Figure 7.9. This is an interaction between the stochasticity of the commons and the mutation rate of the DMs. Figure 7.9 shows that the commons persists longer (on average) when $m r$ is high (0.1), regardless of whether Stoch is low (Stoch $=0$, the dashed line) or high (Stoch $=2$, the bold line).

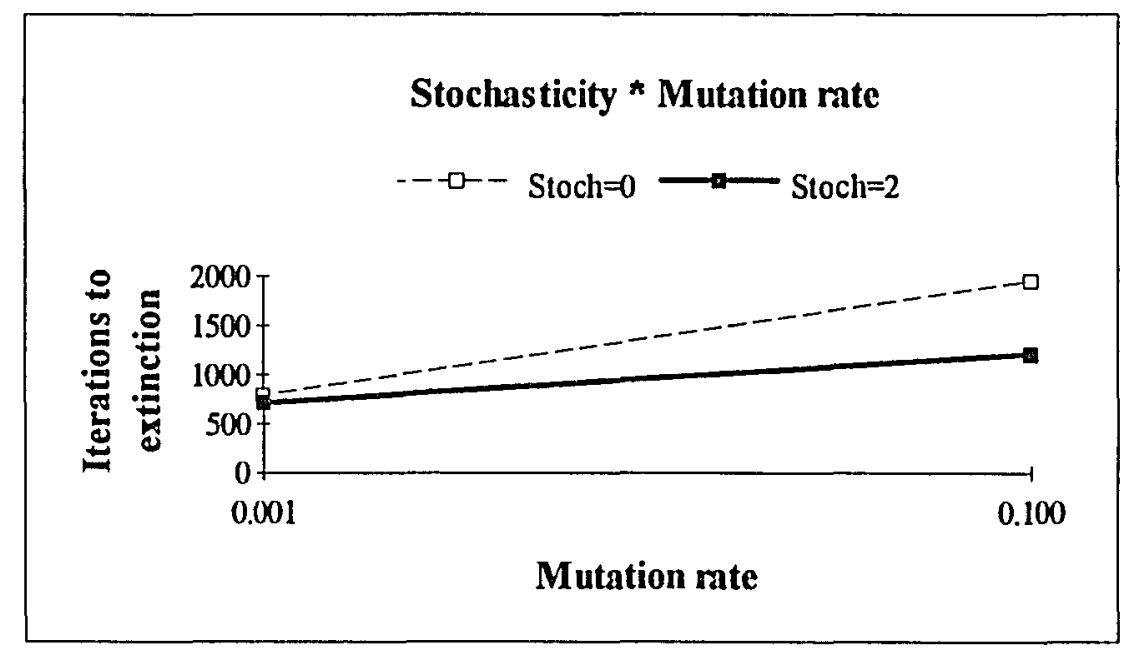

Figure 7.9. Interaction plot for stochasticity of the commons Stoch and mutation rate $m r$, illustrating how low stochasticity and high mutation rate contribute an effect that is in addition to their single-factor effects.

However, there is more of a difference in performance between the high and low Stoch trials at high $m r$ than at low $m r$. This means that Stoch and $m r$ terms not 
only have the single-factor effects described above, but they also interact to add an additional synergistic "bonus" for low Sloch and high mr. Although the two lines are not parallel, they both have positive upwards slopes and thus act more or less as complements. In other words, low commons stochasticity is beneficial on its own because it makes the commons behave more predictably, and a high DM mutation rate is beneficial on its own because it keeps a variety of types of DMs in the population; but the two parameter settings are even more beneficial in combination.

TSProb also interacts with $m r$ (standardized $\hat{b}=-1.0, p<0.0005$ ), as shown in Figure 7.10.

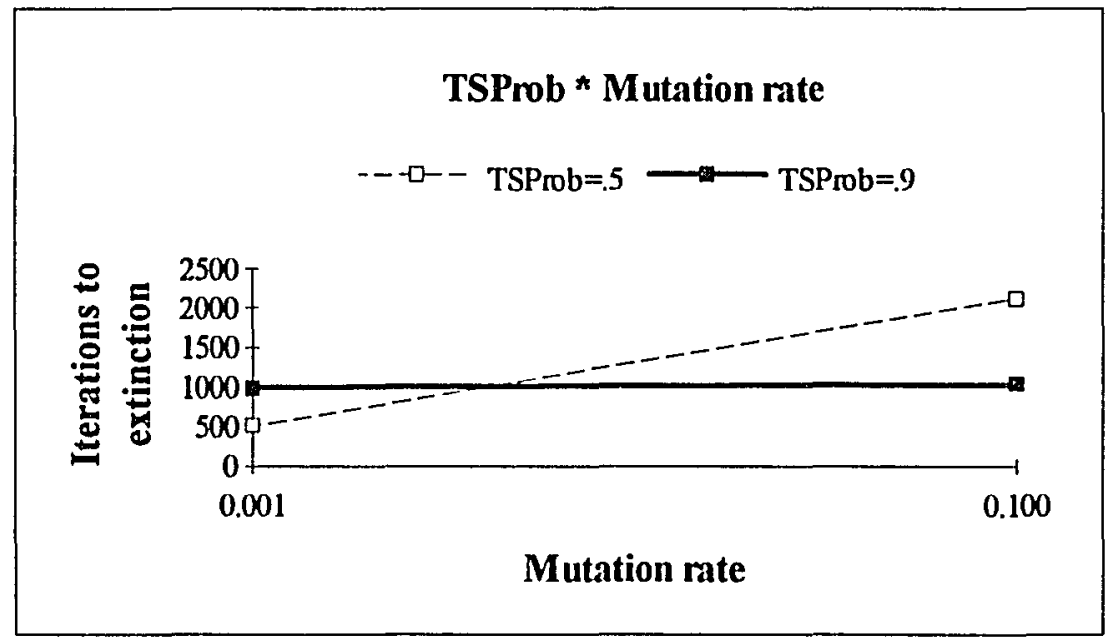

Figure 7.10. Interaction plot for TSProb and $m r$, illustrating a "crossing" interaction that can tend to hide single-factor effects. When population diversily is low (TSProb is high), the effect of the mutation rate is canceled out. When diversity is high, the beneficial effect of high $\mathrm{mr}$ is amplified, but so is the harmful effect of low $\mathrm{mr}$.

Figure 7.10 shows a different kind of interaction, called a "crossing" interaction. Recall that when the parameter that controls progenitor diversity TSProb is high, DM diversity tends to be low because higher-fitness individuals have a higher probability of 
being progenitors to the next iteration of DMs. For low-diversity populations (high TSProb, shown bold in Figure 7.10), the average number of iterations to extinction is essentially the same for high and low $m r$ (i.e., the bold line is flat); but when high diversity is maintained by a low TSProb, $m r$ does make a difference: the beneficial effect of low TSProb amplifies the beneficial effect of high $m r$ : the dashed line slopes upward (this is also illustrated in Figure 7.7, above). At the low mr end of the graph, however, the harmful effects of high TSProb and low $m r$ also act together, this time to produce worse results than either factor would have done alone. TSProb maintains diversity by keeping less "fit" progenitors in the population from one iteration to the next, and $m r$ maintains diversity by randomly changing alleles. When TSProb is low, selection pressures rapidly destroy whatever diversity $m r$ occasionally (re)introduces into the population.

The Stoch $*$ TSProb interaction (standardized $\hat{b}=0.5, p=0.002$ ) shown in Figure 7.11 is another crossing interaction. When Stoch is high, the effect of TSProb tends to be canceled out (the bold line in Figure 7.11 is nearly horizontal); but when Stoch is low, the beneficial effect of increased population diversity (low TSProb) can be seen.

The TSProb $* m$ interaction (standardized $\hat{b}=1.8, p<0.0005$ ) is also crossing in nature, although the slopes of the high diversity $($ TSProb $=0.5)$ and low diversity $($ TSProb $=0.9)$ lines are both upward (see Figure 7.12).

Similarly to the profit function parameter $k$, the "Conscience" function parameter $m$ sets the slope and $y$-intercept for the "Conscience" function: when $m$ is relatively low (0.02), "Conscience" is not as important to DMs as when $m$ is high, and the commons tends to go extinct faster. For low $m$ trials such as those shown in Figures 7.5, 7.6, and 7.7 at the beginning of this section, high-diversity (low TSProb, 
dashed line) populations do better than low-diversity populations. But when $m$ is relatively large, the commons lasts longen on average regardless of TSProb, and the effect of TSProb disappears. This means that when "Conscience" becomes more important to most DMs, they show more of a tendency to Refrain. Diversity in the population is then not as important as when some DMs are less driven by "Conscience."

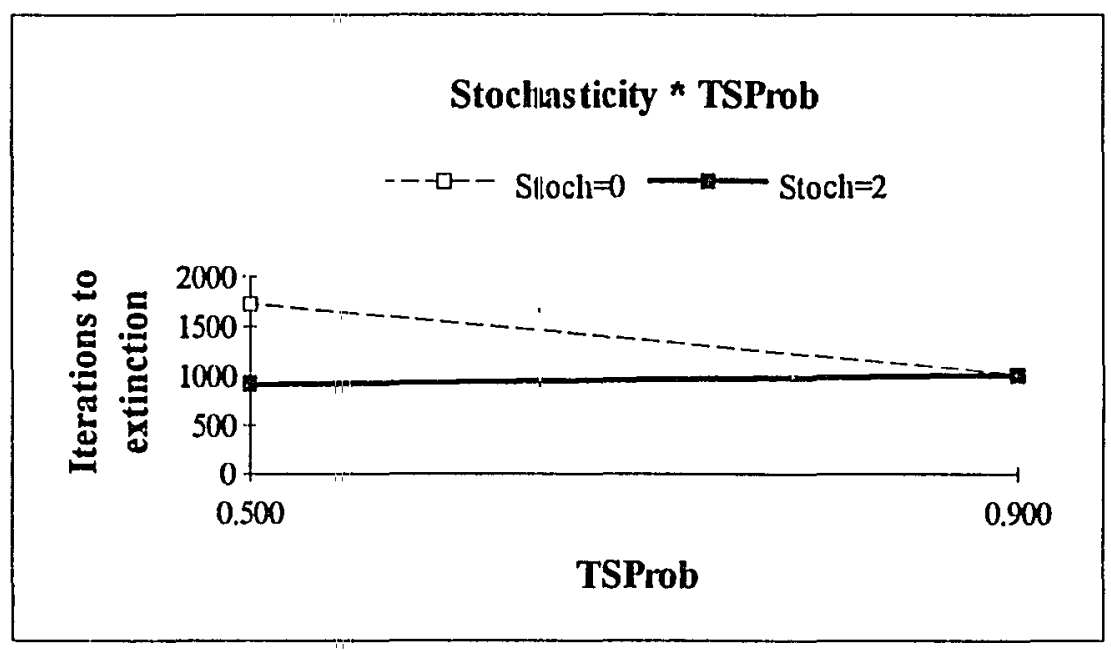

Figure 7.11. Interaction plot for TSFirob and Stoch, showing how when TSProb is high (diversily is low), the /beneficial effect of low Stoch is canceled out.

The three-factor interaction TSProb*k*m (standardized $\hat{b}=-1.4, p<0.0005$ ) cannot be shown in a two-factor plot. Three-way interactions are harder to visualize or interpret than two-factor interactions, but the general idea of how they work is the same: whenever a regression involves significant interaction terms, it means that combinations of settings for the variables involved produce synergistic contributions beyond the contributions of lower-order effects alone. For simplicity's sake, it is generally preferable to find an acceptable model that does not include interactions 


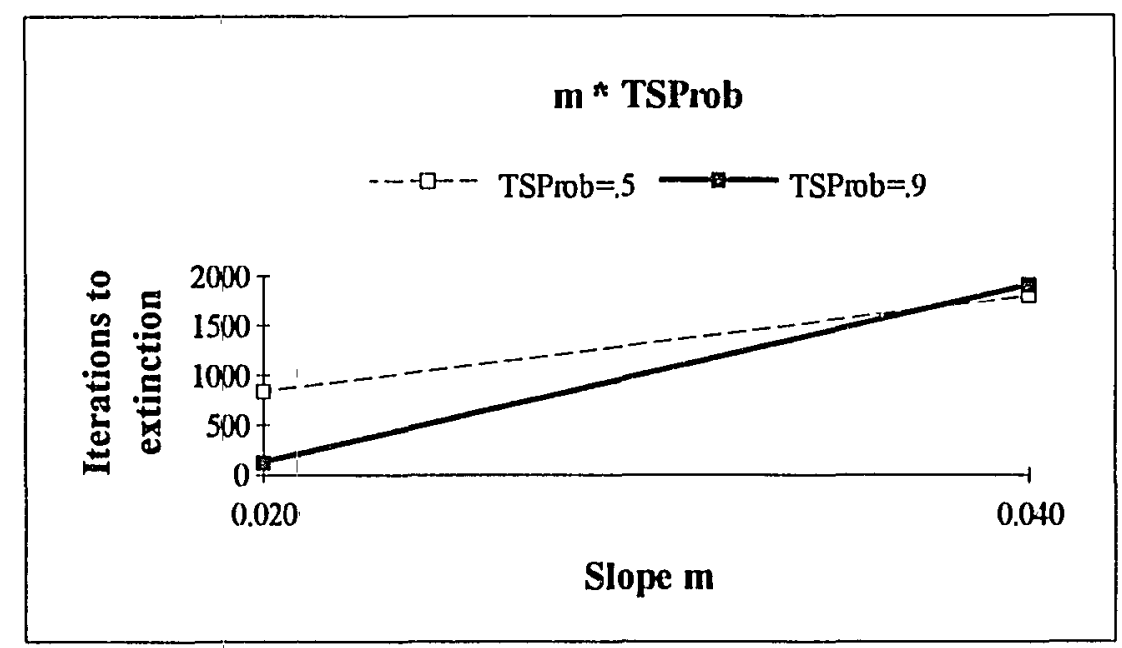

Figure 7.12. $m *$ TSProb interaction, illustrating the somewhat-crossing interaction between the parameter controlling most of the DM population diversity TSProb, and the parameter that determines the magnitude and slope of the "Conscience" function $m$. When "Conscience" is relatively important $(m=0.04)$, population diversity TSProb has little effect on commons persistence; but when "Conscience" is less important $(m=0.02$ ), the higher diversity populations (lower TSProb, dashed line) last longer than the lower diversity populations.

beyond two factors (note that since there are five single factors, interactions could involve as many as five variables). However, the best regression that could be found without a three-way interaction produced $R_{a d j}^{2}$ of only 0.66 . The ANOVA shown in Table VI was the best that could be found, according to the objectives outlined above:

- Reasonableness: the contributions of Stoch, TSProb, $m r$, and $k$ are consistent with intuitive expectations: the commons lasts longer when stochasticity is low, diversity is high, and the profit function is less steep and lower in maximum magnitude. Although the "Conscience" function parameter $m$ is included in two of the significant interaction terms (TSProb*m and TSProb*k *iil), it is not significant as a main effect, probably because it is involved in 
crossing interactions (crossing interactions tend to "cancel out" or hide main effects).

- Explanatory power: The model chosen has the maximum $R_{a+j}^{2}(0.81)$ and minimum MSE (628.4) for any models that include only variables with $p$ values less than 0.015 , and no interaction terms greater than third-order.

- Validity: the plots (not shown) of residual terms, versus estimates, exhibit good uniform scatter with no obvious patterns that could indicate inequality of variance, and the normal probability plot (not shown) is quite straight. No outliers (studentized residual $>2.5$ ) were identified for the model chosen.

\section{Conclusion}

The simulations in the next chapter will further explore what this model suggests about TOC solutions, but the experimental findings discussed above already suggest that the following elements are necessary for a commons management system to be workable, at least in the hypothetical system modeled by this simulation:

- Stochasticity of the commons (Stoch) must be low enough, and/or forecasting accurate enough, to allow planning and decision making to be effective. Technological means of reducing stochasticity sometimes yield the hoped-for results, but in general, healthier ecosystems tend to not be as sensitive to disturbances as weakened systems. The most effective means for dealing with the possibility of erroneous forecasting is to make sure the system being forecasted is as healthy as possible. Even if the forecasts are wrong, the system then has enough resilience to recover. 
- There must be enough diversity of beliefs and values that the population can respond in time to surprises. Diversity is maintained when a society protects and perhaps even encourages contrary viewpoints (via TSProb in the simulations), and when it chooses to invest in efforts such as funding research and higher education, that work to constantly (re)introduce diversity (via $m r$ in the simulations).

- Some kind of payoff must provide enough of a benefit that some people will choose to Refrain because they receive more from Refraining than profit provides them for Taking. This payoff could be something like the "Conscience" function $C(x)$ in the simulations; it could also be a monetary payoff such as a tax credit or payment.

- Demand must be elastic enough (low profit function slope $k$ in the simulations) that, in contrast to what Hardin claimed, people feel they have a choice, and do not feel compelled to Take regardless of the temptation from profit.

Although the factorial experiments were all run with constant population sizes, the importance of the profit function parameter $k$ implies a rather discouraging conclusion about the impacts of increasing population. Increasing $k$ in the experiments leads to commons destruction, regardless of the settings for other variables. Recall from the previous chapter that the slope and magnitude of the profit function increase with increasing population; thus, if population increases, so does $k$. Parameters that help prevent commons destruction (the "Conscience" function parameter $m$, and the diversity components TSProb and $m r$ ) are not affected by increasing population size. Since the impact of profit increases while the impacts from other parameters do not, there is little to counteract human greed as it grows with human population. 
The fact that demand grows with population is built into the mathematical model and thus actually dictates this outcome - but demand increasing with population is also consistent with the real world: profit is a function of supply and demand, which are functions of absolute as well as relative scarcity. As population grows, a resource becomes not only scarcer in an absolute sense because of consumption, but also relatively scarcer because of increased numbers of would-be consumers. There are more DMs choosing to Take with increasing population for two reasons: there are more people making decisions because there are more of them, and Taking becomes relatively more attractive to almost all DMs as the population grows (due to increașed $k$ ).

Perhaps the most interesting, and unexpected, finding of these experiments was the importance of population diversity. This finding suggests that Ashby's (1956) Law of Requisite Variety (LRV) might apply to these simulations. Ashby argued that, in order to be effective, the controller for a cybernetic system must be able to absorb at least as much variety ${ }^{9}$ as the system can produce: only variety can absorb (or "control") variety. Ashby was concerned with cybernetic systems with feedback control in a very general sense, and his LRV can be applied to many kinds of real systems. In this model, the GA "feeds back" to the DMs the effects of their actions on the commons. The DMs act as the controller, and the commons is the system being controlled. Consistent with Ashby's law, only by maintaining a high enough level of variety themselves (via $\mathrm{mr}$ and TSProb) can the DMs respond effectively to changes in the commons. When stochasticity is higher in the commons, it means the variety of the system being controlled is higher, at least over a particular time period. Then the DMs require higher

9 Ashby used "variety" to indicate the number of states a system could exhibit. 
$m r$ and/or TSProb in order to respond effectively: hence the significance of the interaction terms Stoch * $m r$ and Stoch * TSProb.

The importance of variety in these experiments is also consistent with the Fundamental Theorem of Natural Selection in real evolutionary systems. This theorem states that (Meffe \& Ehrlich, 1993, p. 9):

...'the rate of increase in fitness of any organism at any time is equal to its additive genetic variance in fitness at that time.' This means that the ability to adapt to changing circumstances is directly proportional to the amount of genetic diversity carried in a population. Loss of diversity is equated with loss of adaptability. A good working guideline, then, is that maintenance of genetic diversity in populations is good, and losses of diversity through drift, inbreeding, or other means, is bad. 


\section{Chapter V III}

\section{PHASE III: POTENTIAL SOLUTIONS}

\section{GAME THEORY ANALYSIS}

EMAV decision trees and game theory matrices provide different insights into decision processes. In decision trees, actions by others (including Nature) are represented by probabilistic event nodes, and the outcomes of these event nodes are only important insofar as they determine the payoffs received by the DM. In game theory, payoffs others will receive are important in their own right because they promote an understanding of the competitive and cooperative characteristics of the situation. Probabilities appear only if it is assumed that a player is using a mixed strategy. Although both game theory and decision theory rely on a strict definition of "rationality" on the part of all decision-makers, in game theory assumptions must be made about what kind of decision rule other players are following (e.g., maximin, minimax regret, difference maximization, or mixed maximin). EMAV decision theory generally seeks to maximize expected utility; game theory seeks to identify the best strategy for a particular decision rule. 
This analysis was motivated by Henry Hamburger's (1973) argument that commons dilemmas may not always be PDs. He argued that while many social dilemmas could be modeled as N-person PDs, conservation dilemmas may better be modeled as N-person games with elements of Chicken, or as "compound" games where different players play different games against one another (Hamburger, 1973).

Discussions of environmental issues in the literature and in casual conversation suggest that some people do express preferences consistent with the N-person PD. However, others sound more like they perceive a game of Chicken because they see total destruction as being the worst possible outcome, instead of the second-worst outcome (see Figure 8.1). If this is so, it has important implications for solutions to environmental commons dilemmas, because Chicken, and its variations, do not have the non-pareto-optimal, dominant strategy of the $\mathrm{PD}$, and are thus theoretically easier to solve.

\begin{tabular}{|c|c|c|c|c|c|}
\hline \multirow[b]{3}{*}{ DM Refrains } & \multicolumn{2}{|c|}{ Other player } & & \multicolumn{2}{|c|}{ Other player } \\
\hline & Refrain & Take & & Refrain & Take \\
\hline & $2 a$ & $b_{b} 0$ & DM Refrains & 2 & 1 \\
\hline DM Takes & $3 c$ & $\begin{array}{ll}d & 1\end{array}$ & DM Takes & 3 & 0 \\
\hline
\end{tabular}

Figure 8.1. Two-person $P D$ and Chicken, showing ordinal payoffs to row player (where " 3 " is best and " 0 " is worst). Cells are labeled $a, b, c, d$. "DM" is Decision Maker. PD and Chicken are symmetric by definition, and thus payoffs are the same to the column player. 


\section{Kinds of games}

There are two fundamental differences between the PD and Chicken: (1) the PD has a strongly dominant, non-pareto optimal strategy (always Take), whereas in Chicken, a player's optimal choice is to do the opposite of what other players do; and (2) if the outcome is total destruction of the commons, a PD player will still prefer Taking over Refraining, whereas a Chicken player's preference will be the reverse (see Figure 8.1).

The preference orders for the two games are:

PD: $c>a>d>b$

Chicken: $c>a>b>d$

Since Taking is dominant in the PD, any ordinary decision rule will produce the same result. However, with no dominant strategy, a Chicken player must pick a decision rule. In the absence of knowledge about what the other player will do, the most conservative choice would be to adopt a maximin strategy. A player who uses maximin would choose to Refrain because that way, the least bad "worst case" possibility would be chosen. In contrast, an optimist using an expected value decision rule would Take, because of an expectation that others would Refrain. A pessimist would Refrain. Maximin is consistent with the pessimists' choice, but not with the optimists' choice, and so if there is some rational reason for expecting a favorable situation, maximin could be an irrationally cautious rule for optimists. There are other 
decision rules that could be used, such as minimax regret, or difference maximizing, but a typical game theorist's recommendation would be to pick the dominant strategy, where possible, and use maximin if necessary (Hamburger, 1979). The other workhorse of decision-making is EMAV's expected value rule. Only the three most commonly recommended rules are examined here (pick the dominant alternative, maximin, and maximize EMAV).

The games shown in Figure 8.1 are two person games, whereas there are so many DMs in the TOC that any underlying games may be considered N-person games. It is customary to illustrate the payoffs in symmetric N-person games with graphs, where the payoffs for Taking (usually called "Defecting") and Refraining (usually called "Cooperating") depend on the number of total Refrainers $R$ and the maximum possible number of Takers $T_{\max }$; the number of Takers is then $T_{\max } \operatorname{minus} R$. Although only ordinal payoffs such as those in Figure 8.1 are required to define the two-person PD and Chicken, at least interval payoffs are required to create the graphs. Two-person symmetric interval game payoffs can be converted to $\mathrm{N}$-person compound game payoffs as (Hamburger, 1973):

$$
\begin{aligned}
& \bar{V}(\text { Refrain })=(a-b) R+b T_{\max }-a \\
& \bar{V}(\text { Take })=(c-d) R+d T_{\max }-d
\end{aligned}
$$

where $a, b, c$, and $d$ (see Figure 8.1) are interval payoffs, $\bar{V}$ (Refrain) is the payoff for Refraining, and $\bar{V}$ (Take) is the payoff for Taking. 
For example, if the ordinal payoffs $0,1,2,3$ shown in Figure 8.1 were actually interval payoffs $0,1,2,3$, and $T_{\max }$ were 100 , then the payoff graphs for an $\mathrm{N}$-person PD and an N-person Chicken game would look like the graphs in Figure 8.2. Note that $\bar{V}$ (Refrain) is undefined if there are no Refrainers, and $\bar{V}$ (Take) is undefined if there are no Takers.
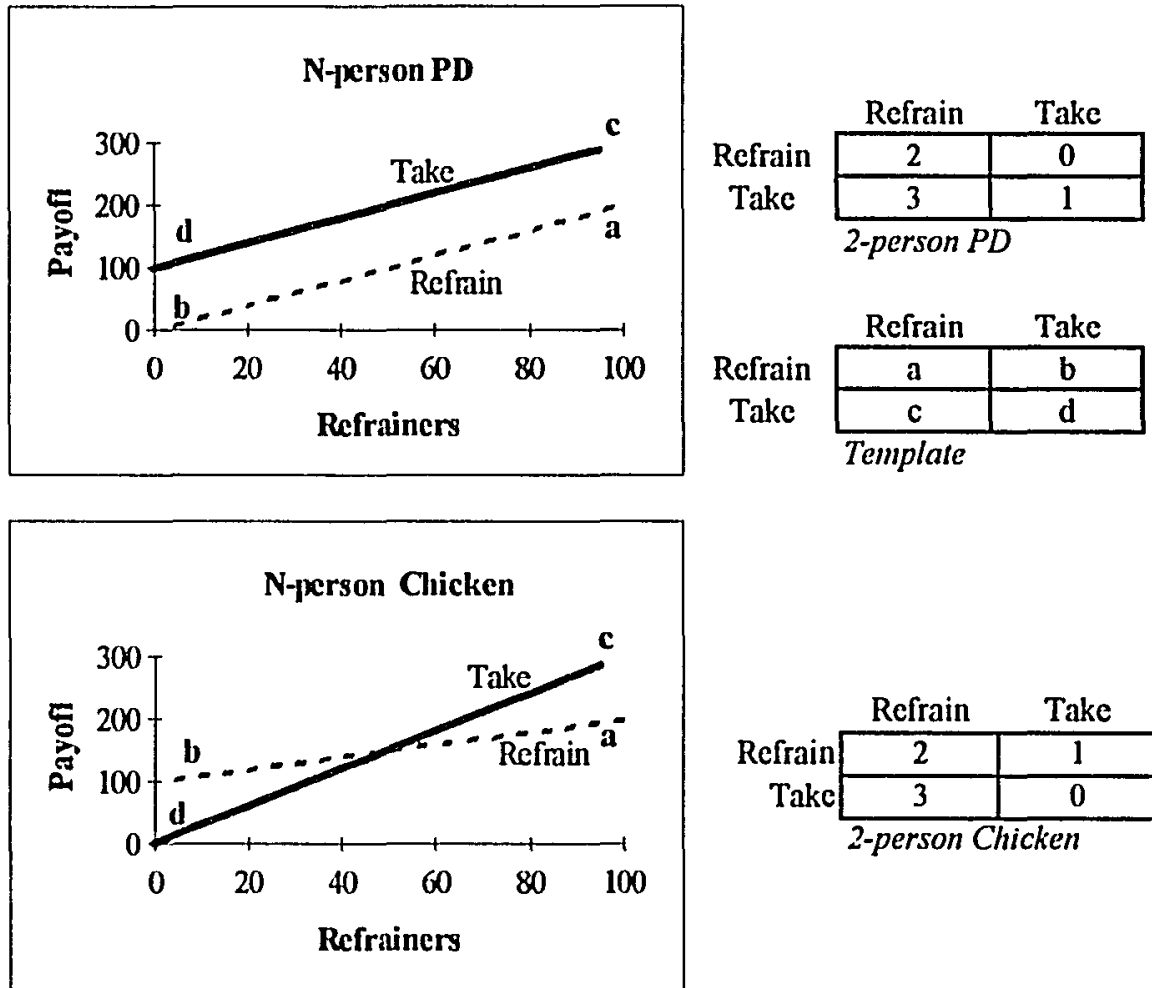

\begin{tabular}{|c|c|c|}
\hline & Refrain & Take \\
\hline Refrain & 2 & 1 \\
\hline Take & 3 & 0 \\
\hline
\end{tabular}

Figure 8.2. Payoff graphs of PD and Chicken. The vertical axes show the payoffs to a particular player for Taking or Refraining, given the tolal number of Refrainers shown on the horizontal axis. The payoffs to each player are calculated using Eq. (8.1). The matrices show the interval payoffs from the two-person games that underlie the $\mathrm{N}$-person payoff calculations. The labels $a, b, c, d$ on the graphs correspond to the cells in the matrices, as shown in the "Template" matrix. Note that $\bar{V}$ (Refrain, 0 Refrainers) and $\bar{V}$ (Take, 0 Takers) are undefined. 
The two most important characteristics that define an $\mathrm{N}$-person PD graph are (Hamburger, 1979):

- $\bar{V}($ Take $)>\bar{V}$ (Refrain): the Taking graph is always above Refraining graph: $c>a$ and $d>b$ : Taking is the dominant strategy.

- $\bar{V}$ (Refrain), when all Refrain $>\bar{V}$ (Take), when none Refrain: the right end of the Refrain graph is higher than the left end of the Take graph: $a>d$ : The dominant strategy produces a deficient outcome.

The most important characteristics that define an N-person Chicken payoff graph are:

- $\bar{V}$ (Refrain), when most Take $>\bar{V}($ Take $)$, when most Take: $b>d$, opposite of PD.

- $\bar{V}$ (Take), when móst Refrain $>\bar{V}$ (Refrain), when most Refrain: $c>a$, same as PD.

- $\bar{V}$ (Refrain), when all Refrain $>\bar{V}($ Take), when none Refrain: $a>d$, same as $\mathrm{PD}$.

The main indicator that distinguishes the two graphs is the fact that in the PD, the $\bar{V}$ (Take) and $\bar{V}$ (Refrain) graphs never cross, and $\bar{V}$ (Take) is always above $\bar{V}$ (Refrain). In Chicken, the graphs must cross somewhere because in Chicken, a player's preference depends on how many other players Refrain. This is equivalent to saying that there is no dominant strategy in Chicken, whereas there is in the PD, just as 
is the case for two-player games. The crossing point of the Chicken graphs indicates a point of theoretically globally stable equilibrium because Taking is favored when the number of Refrainers is more than those at the crossover point, and Refraining is favored when the number of Refrainers is less than those at the crossing point. If this equilibrium point could be identified, and everyone could agree on who the Takers and Refrainers were to be, then this equilibrium would represent a theoretical solution to an $\mathrm{N}$-person game of Chicken. Unfortunately, the requirement that the crossover point be known, and that everyone must agree on who Takes and who Refrains, is exactly why this kind of solution is so problematic in the real world.

Two other games that are similar to Chicken, in that they have the same two stable equilibria, are Hero and Benevolent Chicken (BC) (see Figure 8.3).

In Chicken, Benevolent Chicken, and Hero, if all players do the same thing, they all receive either the worst or next-to-worst outcome. In Benevolent Chicken, the " 1 " and "2" mid-range payoffs of Chicken are swapped. In Benevolent and ordinary Chicken, a player's first choice is to Take and have the others Refrain. In Chicken, the payoff for Refraining is greater if others Refrain than if they Take $(a>b)$. In Benevolent Chicken, a player who Refrains magnanimously prefers that the opponents Take: presumably the player is benevolent enough to prefer that at least some players get the maximum payoff, even if that player is not in the more fortunate group.

In Hero, the "2" and "3" payoffs of Benevolent Chicken are swapped. A Hero player's first choice is to be the only Refrainer. Hero is the game played by a certain 
type of member of a crowd watching a person drowning. If everyone else stands around watching (i.e., Taking the easy way out), then a player who jumps in to rescue the drowning victim gets to be a hero (i.e., by Refraining from taking the easy way out). If someone else jumps in first, this player would prefer not to jump in the water after all, so that, as in Chicken, $b$ and $c$ are both stable equilibria.

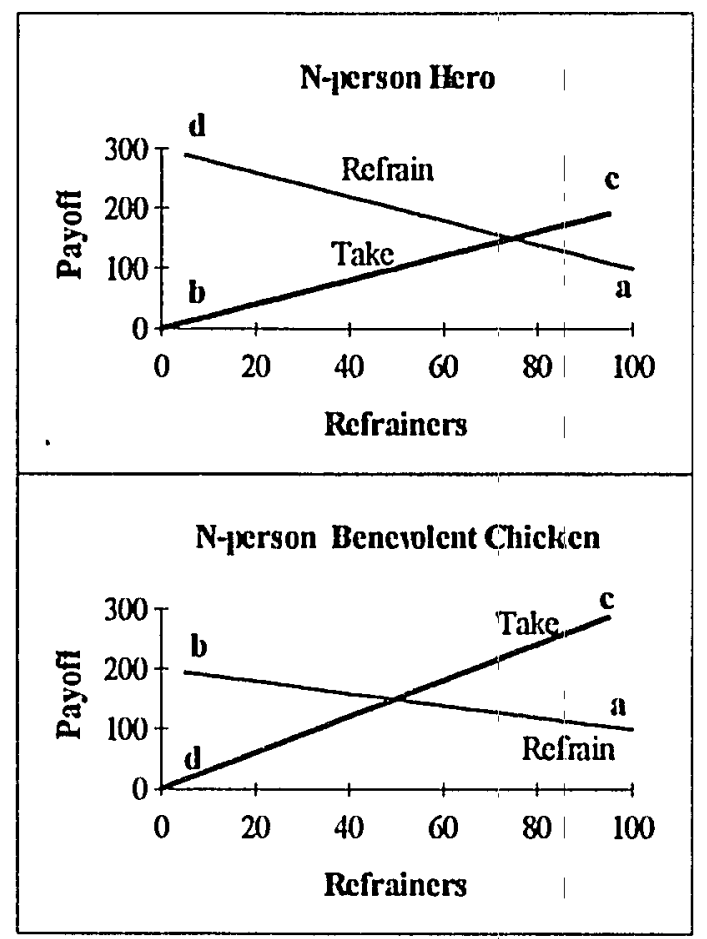

\begin{tabular}{|c|c|c|}
\hline & Refrain & Take \\
\hline Refrain & 1 & 3 \\
\hline Take & 2 & 0 \\
\hline
\end{tabular}

\begin{tabular}{|c|c|c|}
\hline & Refrain & Take \\
\hline Rcfrain & 1 & 2 \\
\hline Take & 3 & 0 \\
\hline
\end{tabular}

Figure 8.3. Payoff graphs and two-person games of Hero and Benevolent Chicken.

Hero, Chicken, and Benevolent Chicken are tricky dilemmas because if everyone tries to get their best payoffs, they will all get their worst (Chicken and Benevolent Chicken) or next-to-worst (Hero) payoffs. In order to achieve one of the two pareto-optimal outcomes, all players cannot make the same choice. It is interesting 
that a worse outcome would result for these three games if all the players were following such idealistic guidelines as Kant's imperative (act so that if everyone's actions reflected the rule underlying your act, everyone would be better off) or the Golden Rule, than if there were a good mix of selfish opportunists and virtuous idealists. Communication can help resolve these games, unlike the PD (because of its dominant strategy), although the situation is also sensitive to threats and lies. Although symmetry is assumed to apply by definition in the games named above, Hamburger (1973) points out that in N-person games, a play'er could be simultaneously playing several different games against other individual players. Then the payoffs illustrated by the graphs for a particular player would be the sums of payoffs of multiple two-person games. If symmetry is assumed, then all players have the same payoffs and the calculations are straightforward; but there is no straightforward way of representing $\mathrm{N}$-person games where each player is simultaneously playing different games against other players, let alone if the game is a Hobbesian "Warre of All against All" (Hobbes, 1649). As argued earlier, assuming pairwise interactions in order to represent $N$-person games becomes meaningless if there is a large number of players.

It is difficult to imagine that all the players in the TOC could have the same payoff graphs, or be expecting that others had the same payoff graphs, let alone interacting pairwise. In fact, it is difficult to imagine that any player in the TOC in general knows what the rules are perceived to be by the other players, let alone what their payoffs might be. Any DM's payoffs, based on the calculations of the previous 
chapters, could be represented by graphs as shown above; but unless symmetry is assumed, no assumption can be made about any games that might underlie those graphs.

Hamburger deals with this problem by showing how one player playing different pairwise games simultaneously against other players can have payoff graphs that look like N-person PD or Chicken graphs (Hamburger, 1973). While it is difficult to know what to call such graphs, Hamburger acknowledges that it is nonetheless important to recognize the character of the resulting graphs. He thus the calls any graphs "PD-like" if they exhibit the typical $c>a>d>b$ ordering of the PD, or "Chicken-like" if they exhibit the $c>a>b>d$ ordering of Chicken. The ordering of the endpoints $a, b, c$, and $d$ of the payoff graphs could result from one player playing the same game pairwise against all other players, using Eq. (8.1), or from one player playing different games pairwise against all other players, or simply from outcome calculations based on how many Take and how many Refrain, without making any lassumptions about other players' payoff graphs. Each player's payoff graph may in fact be unique, and if the ordering is $c>a>d>b$, consistent with the $\mathrm{PD}_{n}$ it would be called "PD-like" ; if it $c>a>b>d$, consistent with Chicken, it would be called "Chicken-like".

The same approach is followed here. That is, if the payoff orderings in a payoff graph are consistent with the ordering of, say, the $\mathrm{PD}$, the graph may be referred to as "PD-like." It is impossible to tell from the graph whether the DM is actually playing the $\mathrm{PD}$, because the PD is defined by pairwise, symmetric payoff matrices and any one 
DM's graph reflects only that DM's payoffs, given the range of potential numbers of Refrainers used to calculate the outcome. It only means that the payoffs for that DM are ordered in such a way that if the game were completely symmetric (all players "see" the same graph), then it would be an N-person PD. The implication then is that Taking will be dominant for that player, and that if most players Take, then players with the PD-like ordering will be worse off than they would have been if most had Refrained. Note that if other players have different payoff graphs, so that, say, Taking is dominant for them but is also pareto optimal, everyone would still Take - but the players with the PD-like graphs would feel worse off than they would have if all had Refrained (a deficient ontcome), while the other kind of players would not (not a deficient outcome). The difference in optimality of outcomes for players with different payoff orderings can easily be shown in $2 \times 2$ game matrices, because the "We both Take" cell can be compared to the "We both Refrain" cell. N-player graphs only show the optimality af outcomes for players whose payoffs are represented by those graphs.

\section{TOC games}

In order to answer the question, "What kinds of games are people playing in the TOC?" it is helpful to simplify the decision trees used in the EMAV simulations (see Chapter VI, Section 4). Since the condition of the commons is strongly dependent on actions by the population as a whole ${ }^{10}$, the double event nodes from the decision trees

\footnotetext{
${ }^{10}$ The commons condition is also determined by its stochastic regeneration function, but in the game theory analysis stochasticity is ignored.
} 
in the decision model section ("Number of Takers" and "Condition of commons") are combined into the single event nodes shown in Figure 8.4.

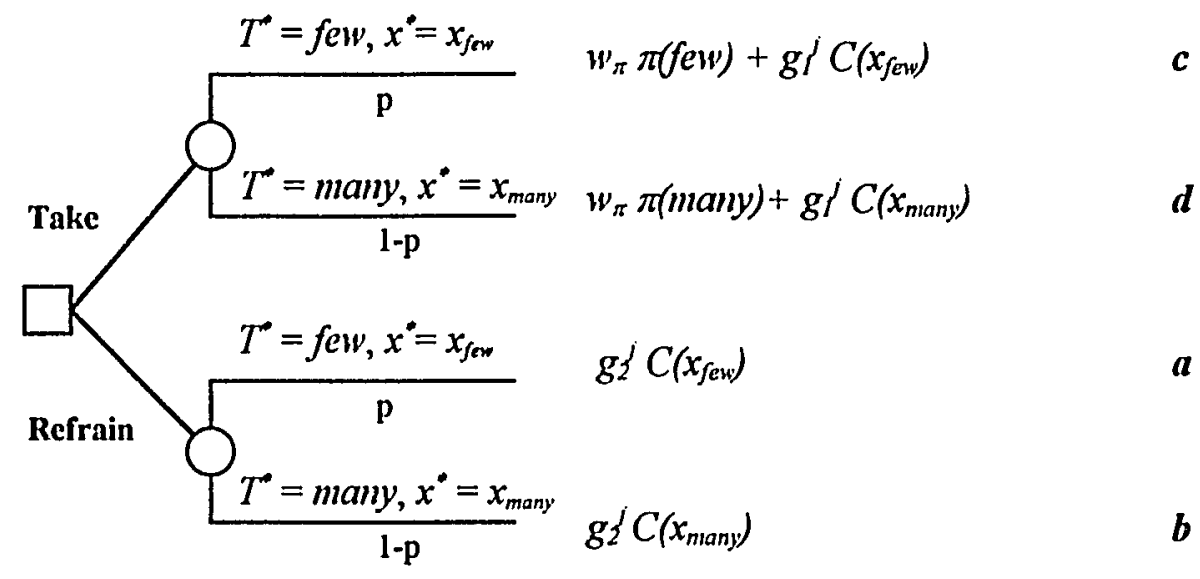

Figure 8.4. Simplified decision tree for DM $j$. The choice (Take or Refrain) is indicated by the box on the left, and the event nodes are indicated with circles: either $T$ = "few" (few Take, with probability $p$ ) and the commons will be in condition $x^{*}=x_{\text {fow }}$, or many will Take (with probability 1-p) and the commons will be in condition $x^{*}=x_{\text {many }} . x_{\text {fow }}>x_{\text {many }}$. The payoffs are listed on the right hand side (see Chapter VI, Section 5), and they correspond to the payoff graph points labeled $a, b, c, d . w_{\pi}$ is the profit function coefficient. The genes $g_{1}{ }^{j}$ and $g_{2}{ }^{j}$ determine the coefficients of the "Conscience" $C(x)$ penalty (for Taking) or reward (for Refraining) for DM $j$ (see Table III). $\pi$ (few) is the profit when few Take, and $\pi$ (many) is the profit when many Take.

One of the fundamental characteristics of a commons dilemma is that any individual DM's actions have negligible effect on the commons or on the choices made by others. This means that the probability estimates in Figure 8.4 are the same for Taking and Refraining.

Because of the conditional independence of the event nodes from the decision, any attribute that has the same value for Taking as for Refraining will cancel out of the 
decision. For example, in ecological economics it is not uncommon for a monotonically increasing, nonconsumable value attribute $N(x)$ to be added to resource management decision trees (Pearce and Moran, 1994). $N(x)$ is dependent on the state of the commons $x$ and represents nonconsumptive activities such as snorkeling to look at fish in a fishing commons, hiking through a grazing commons, or saving a commons for later; existence values such as enjoying knowing that there are wolves in Yellowstone without expecting ever to see one; or saving the commons for later. As a very crude approximation, $N(x)$ is assumed here to be linear, with slope $n>0$ and $y$-intercept of $0 . N(0)$ is assumed to be zero. $N_{\max }$ corresponds to $x=x_{\max }=K$. Thus:

$$
\begin{aligned}
& N(x)=n x+N_{\max } \\
& n=\frac{N_{\max }}{x_{\max }}
\end{aligned}
$$

It is well beyond the scope of this work to determine what values should be used for $N_{\max }$, and thus for $n$. As a first approximation, $n$ is set proportional to $-m$ (the slope of $C(x)$ ) and $N_{\max }$ is set proportional to $C_{\max }$. The impacts of the proportionality constants are discussed later. As was mentioned earlier, $N(x)$ was left out of the decision tree, because it is independent of the choice. Any DM can enjoy the same non-use value regardless of whether that DM Takes or Refrains.

The expected value $E V$ of the nonconsumable value $N(x)$ is a constant on both sides of the decision equation, and hence is irrelevant to the decision (see Figure 8.5):

$$
E V\left\{N\left(x^{*}\right), \text { Take }\right\}=E V\left\{N\left(x^{*}\right), \text { Refrain }\right\}=p w_{n} N\left(x_{f e w}\right)+(1-p) w_{n} N\left(x_{\text {many }}\right)
$$




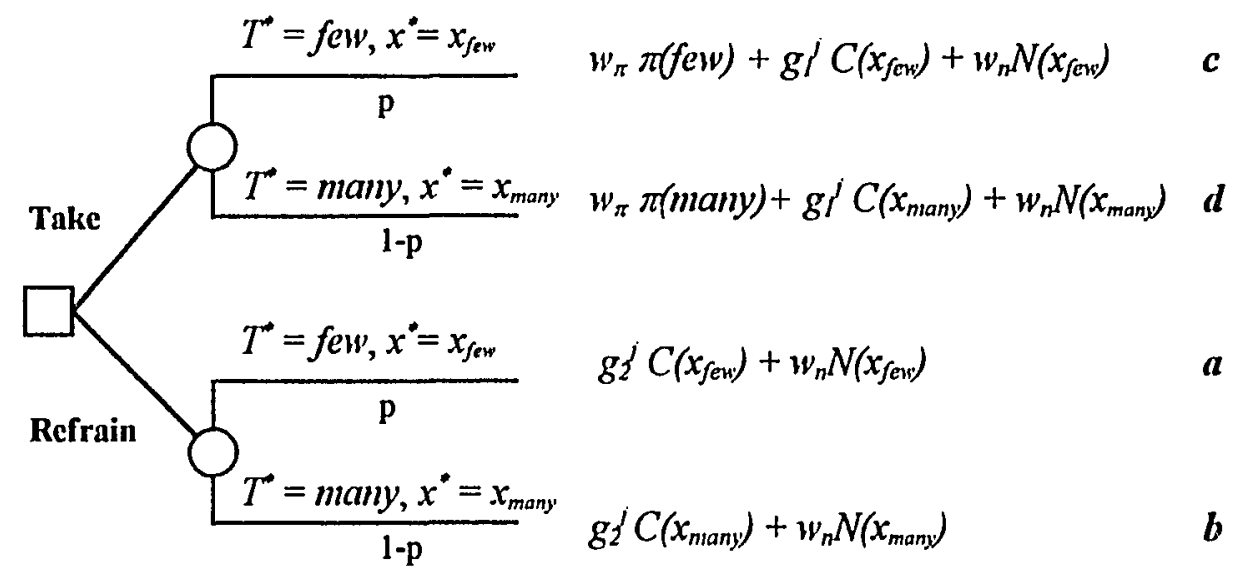

Figure 8.5. Decision tree including nonconsumable use value $N(x)$.

$N(x)$ is far from irrelevant to a game theory analysis, as will be shown below, because only when it, or something like it, is included, can the PD or Chicken arise.

The payoffs shown in Figure 8.5 are the same as those of Type III DMs, with the addition of $N(x)$. Recall that the DM Types are ranked in order of relative importance of "Conscience":

- Type I's have no "Conscience" and always Take;

- Type Il's usually Take but pay a "Conscience" penalty for Taking that becomes important when the commons becomes badly degraded;

- Type III's not only pay a "Conscience" penalty for Taking but also receive a "Conscience" reward for Refraining, and so they Refrain more readily than Type II's; and

- Type IV's always Refrain because they receive no reward for Taking. 
Type III payoffs are used as the base case in the analysis that follows. Types I, II, and IV DMs result when terms are dropped from the Type III model. This fact will be used to evaluate what kinds of games the different DM Types are playing.

The payoffs corresponding to the decision tree of Figure 8.5 are shown in the matrix form of Figure 8.6.

\begin{tabular}{|c|c|c|}
\hline & \multicolumn{2}{|c|}{ Most DMs choose: } \\
\hline & Refrain & Take \\
\hline DM Refrains & $C\left(x_{j}\right)+N\left(x_{j}\right)$ & $C\left(x_{m}\right)+N\left(x_{m}\right)$ \\
\hline DM Takes & $\begin{array}{l}\pi\left(\gamma^{\prime}(w)-C\left(x_{f}\right)\right. \\
+N\left(x_{f}\right)\end{array}$ & $\begin{array}{l}\pi(\text { many })-C\left(x_{m}\right) \\
+N\left(x_{m}\right)\end{array}$ \\
\hline
\end{tabular}

Figure 8.6. Base case payoffs for Type III DMs. The state of the commons when most DMs Refrain is $x_{\text {few, }}$ and the state when most Take is $x_{\text {many }}$. Thus, the "Conscience" payoff when most Refrain is $C\left(x_{\text {for }}\right)$, and when most Take it is $C\left(x_{\text {many }}\right)$. The nonconsumable payoff when most Refrain is $N\left(x_{\text {few }}\right)$, and when most Take it is $N\left(x_{\text {man }}\right)$. Profit when most Take is $\pi($ many), and profit when most Refrain is $\pi(f e w) . w_{\pi}$ and the $C(x)$ coefficients $g_{1}$ and $g_{2}$, though not shown, are implied.

For a homogeneous population with the payoffs of Figure 8.6 to be playing an $\mathrm{N}$-person $\mathrm{PD}$, the weighted payoffs must be such that the preference order is $c>a>d$ $>b$ :

$$
\begin{aligned}
& \pi(f e w)-C\left(x_{f(w w}\right)+N\left(x_{f o n}\right)>C\left(x_{f o n}\right)+N\left(x_{f o w}\right)> \\
& \pi(\text { many })-C\left(x_{\text {man }}\right)+N\left(x_{\text {man }}\right)>C\left(x_{\text {man }}\right)+N\left(x_{\text {many }}\right)
\end{aligned}
$$

The inequalities of Eq. (8.4) will be considered one by one. 


\section{Last inequality of Eq. (8.4): $d>b$}

Starting with the last inequality in Eq. (8.4), note that $N\left(x_{\text {many }}\right)$ can be subtracted from both sides of the inequality, and so it becomes:

$\pi(\operatorname{many})-C\left(x_{\text {man }}\right)>C\left(x_{\text {many }}\right)$

Now, $\pi($ many $)$ is a minimum whereas $C\left(x_{\text {many }}\right)$ is a maximum. This inequality will hold only if the weighted profit payoff when most Take, $\pi(\operatorname{many})$, is greater than the "Conscience" payoff when most Take. Unless Eq. (8.5) holds, the game cannot be a PD: as long as the other inequalities hold, the game will instead be Chicken ( $c>a>$ $b>d)$.

Impacts from "Conscience" increase because of a higher attribute weight, higher $C_{\text {max }}$, or because $x$ is low (the commons is endangered). This means that the inequality will always hold for Type I's because their weight for $C(x)$ is zero; and it will never hold for Type IV DMs, because their weight for $\pi(T)$ is zero. Type III DM payoff graphs (see Figure 8.7 ) are unlikely to show a PD-like ordering because $\pi(m a n y)$ approaches zero while $2 C\left(x_{\text {man }}\right)$ approaches a maximum; for them, $b>d$. Their payoff graphs can look like the N-person Chicken payoff graphs of Figure $8.2(c>a>b>d)$, but not like the PD graphs $(c>a>d>b)$. For Type II DMs, it is possible for $d>b$ as well as $b>d$. Their graphs can thus exhibit both PD-like and Chicken-like characteristics (as long as the other inequalities hold). 
Type II payoffs, $x=7500$, with $N(x)$

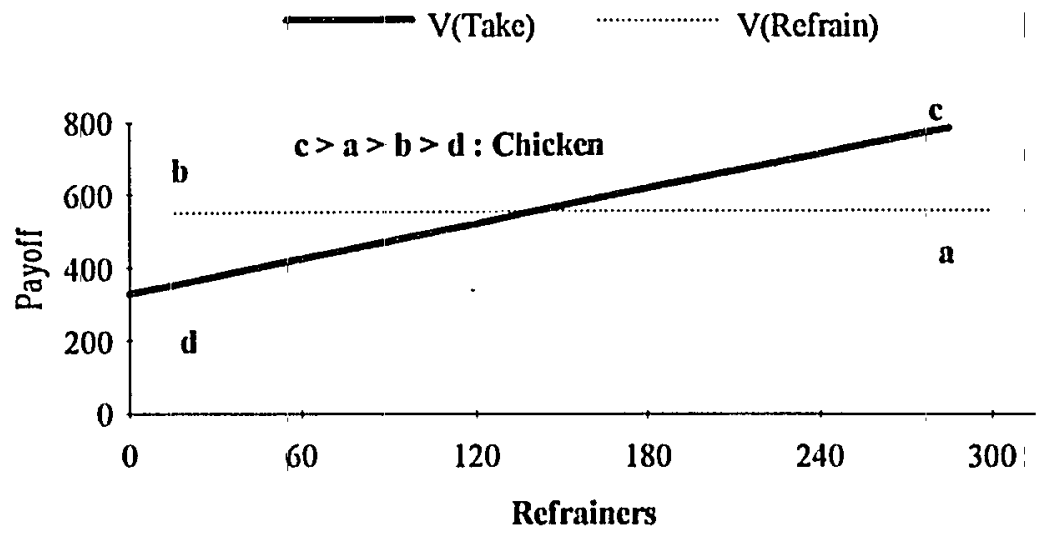

Figure 8.7. Type III payoff graphs, including an appropriate $N(x)$ (discussed below), showing Chicken-like preference ordering $(c>a>b>d)$. Although the dashed graph for the Refraining payoff (line $b-a)$ looks flat, it actually slopes upwards to the right $(a>b)$. The condition of the commons is good $\left(x_{\text {tow }}=7500\right)$. As the commons declines, the intersection of the two lines would move towards the right, indicating a greater tendency to Retirain as the commons declines.

The profit and "Conscience" functions described earlier are used to create these payoff graphs. The nonconsumable value function $N(x)$ was not included in the decision models, but is added here. As a simplification, the regeneration of the commons is ignored and the state of the commons $x$ is decreased by the number of units Taken in the calculations for $C(x)$ and $N(x)$ by replacing $x$ with $x-T$. Thus, for a Type III DM, the payoff graphs are calculated using Eq. (8.6) (see Figure 8.7):

$$
\begin{aligned}
& \bar{V}_{I I I}(\text { Take })=\pi(T)-C(x-T)+N(x-T) \\
& \bar{V}_{I J}(\text { Refrain })=C(x-T)+N(x-T)
\end{aligned}
$$


The payoff graphs for Type II DMs are calculated using the same Eq. (8.6) except that the coefficient for $C(x-T)$ is 0.0 for $\bar{V}$ "(Refrain) (see Figure 8.8).
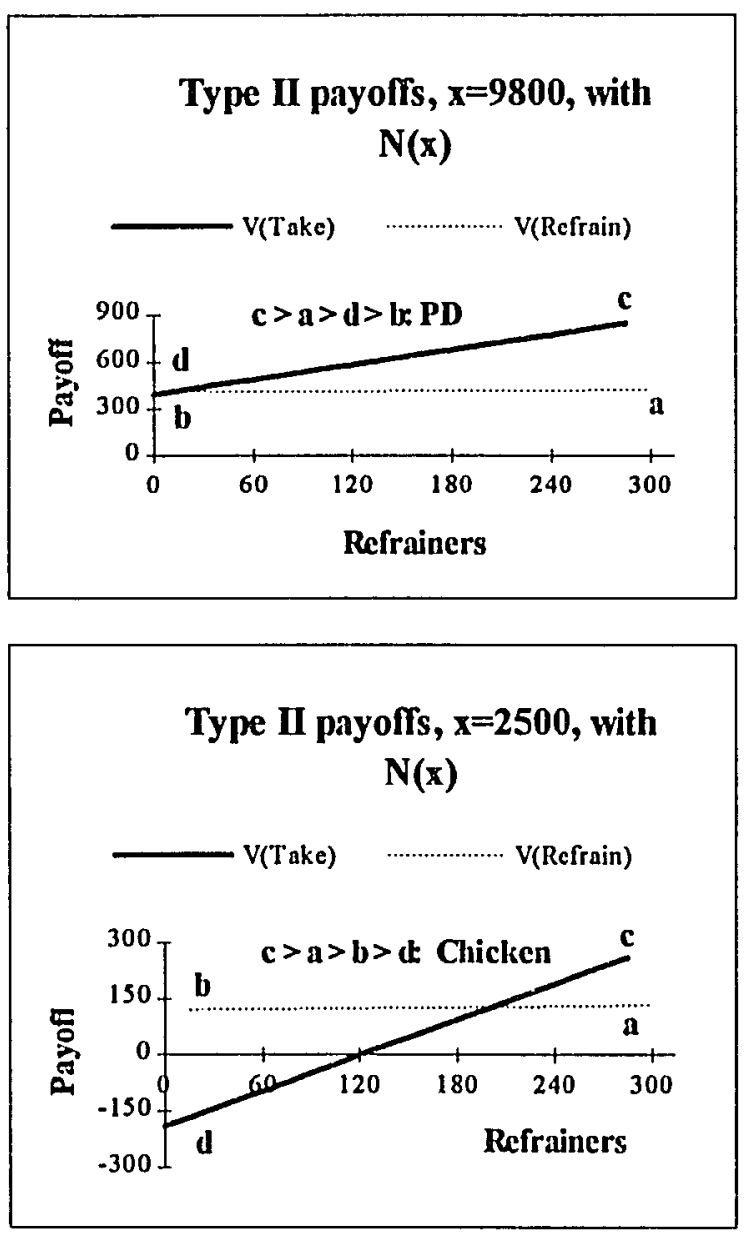

Figure 8.8. Type II payoff graphs, illustrating PD-like graphs when the commons is in good shape $(x=9800)$ but Chicken-like graphs when the commons is in bad shape $(x=2500)$. In the upper graph, although it looks like the two graphs cross, they actually do not, because the payoff to Refrainers when none Refrain (labeled $b$ ) is undefined.

When an appropriate $N(x)$ is included (discussed below) and $x$ is high, the payoff graphs for Type II DMs exhibit the same characteristics as the PD payoff graphs 
of Figure 8.2 (c>a>d>b); but when $x$ is low, the payoff graphs exhibit the same characteristics as the Chicken graphs of Figure $8.2(c>a>b>d)$. If $N(x)$ is not included, Type II payoff graphs cannot be PD-like or Chicken-like, but are instead like the graphs of Benevolent Chicken or Hero.

Recall that the only difference between Chicken and the PD is that in the PD, a player prefers to Take even if everyone else is Taking $(d>b)$ but in Chicken, a player prefers to Refrain if everyone else is Taking $(b>d)$. As long as the worst case outcome is still apparently not too bad, Type II payoff' graphs show the same ordering as the PD: they will always Take. However, if the worst case outcome approaches disaster, they "chicken out" and their payoff graphs are consistent instead with a game of Chicken: they have no dominant strategy.

This is an intriguing result, because the perceived "disastrousness" of the worst case outcome determines whether Type II DMs have a dominant choice or not. The PD-like graph in Figure 8.8 indicates a DM who will always Take, regardless of what others are expected to do. The Chicken-like graph in Figure 8.8 indicates a DM who prefers to do the opposite of what most others do. Thus, in order to decide what to do, a person with the payoffs shown in the bottom graph of Figure 8.8 will need to guess what most other people's graphs look like, and/or will have to pick a decision rule. In contrast, a person with the payoffs of the PD-like graph will not need to guess, and regardless of decision rule, can automatically Take. This is also true for Type I DMs, but neither of the more preservationist Type III's or Type IV's can get into PD games 
because their "Conscience" functions provide a payoff for Refraining.

Middle inequality of Eq. (8.4): $a>d$

Returning now to the inequalities required for a PD in Eq. (8.4), the next inequality to the left in Eq. (8.4) is a little more complex $(a>d)$ :

$$
\begin{array}{r}
C\left(x_{f e w}\right)+N\left(x_{f e w}\right)>\pi(\text { many })-C\left(x_{\text {many }}\right)+N\left(x_{\text {many }}\right) \quad \text { or: } \\
C\left(x_{f e w}\right)+C\left(x_{\text {many }}\right)>\pi(\text { many })+N\left(x_{\text {many }}\right)-N\left(x_{f e w}\right)
\end{array}
$$

$\pi($ many $)$ is a minimum, and $N\left(x_{\text {many }}\right)-N\left(x_{\text {few }}\right)$ is always less than zero. As long as the weighted "Conscience" function has some value, this inequality will probably hold, particularly for the more preservationist Types III and IV. This inequality is required for all four games discussed above, and what it means is that the payoff to each Refrainer if all Refrain is greater than the payoff to each Taker if all Take. This is the source of the dilemma of the PD: Taking is dominant in the PD, and so all will Take; but everyone would be better off if all Refrained.

A PD-like payoff graph is more likely for larger populations made up of Type I's or Type II's because the more such Takers there are, the larger the difference between $x_{f e w}$ and $x_{\text {many }}$ (see. Figure 8.9) For smaller populations, it is more likely that the inequality of Eq. (8.7) will not hold. If Eq. (8.7) does not hold, while the other inequalities do hold, Taking will still be dominant for Types I and II, but it will now be pareto-optimal $(d>a)$. This calls to mind Hardin's historical equilibrium state, where everyone who wanted to could Take, with no problem, because there were few enough villagers that their total harvest remained below the commons ability to regenerate. 

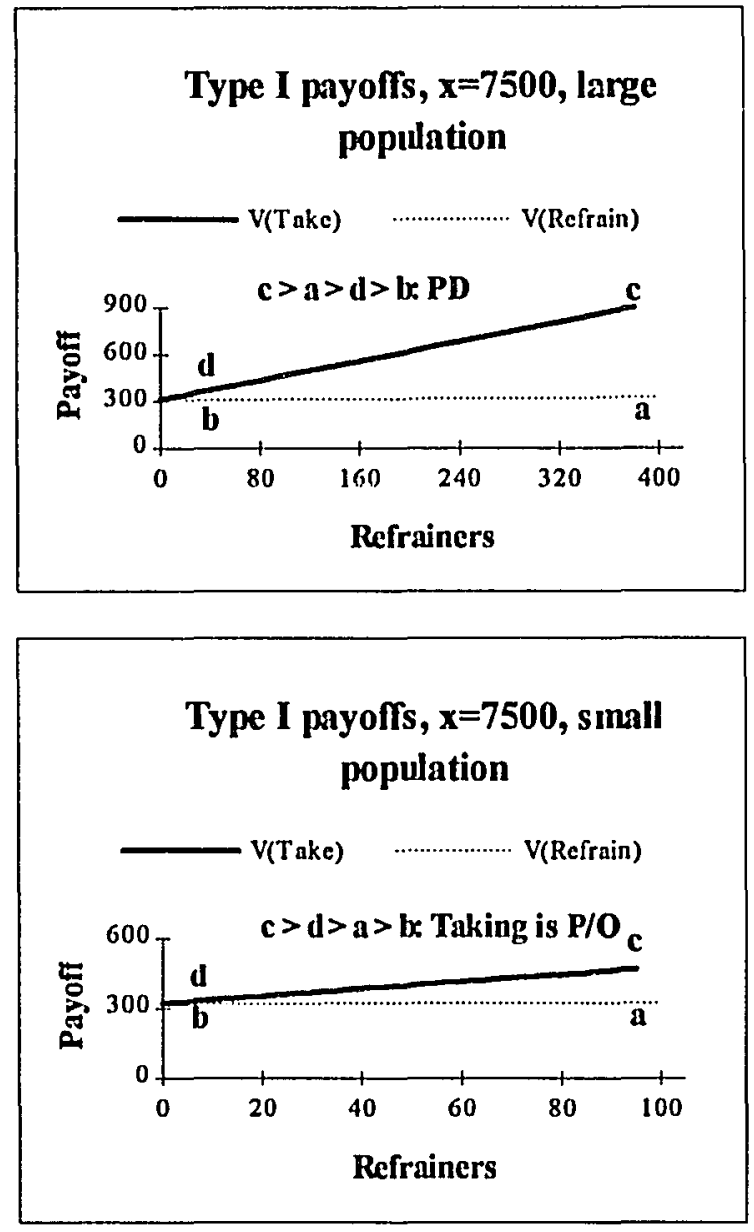

Figure 8.9. Payoff graphs for Type I DMs, including $N(x)$. The upper graph is for a relatively large population (the maximum number of Refrainers = $400)$, and the payoff ordering is PD-like ( $c>a>d>b)$. Taking is dominant but not pareto-optimal. The graph on the bottom is for a relatively small population (the maximum number of Refrainers $=100$ ), and the payoff ordering is not PD-like: Taking is dominant, but because $d>a$, it is also pareto-optimal $(\mathrm{P} / \mathrm{O})$.

First inequality of Eq. (8.4): $c>a$

$N\left(x_{f e w}\right)$ appears on both sides of the first inequality of Eq. (8.4), and so that 
inequality $(c>a)$ becomes:

(8.8) $\pi(\mathrm{few})>2 \mathrm{C}\left(\mathrm{x}_{\mathrm{few}}\right)$

$\pi(f e w)$ is a maximum, while $C\left(x_{f e w}\right)$ is a minimum. As long as the weighted profit payoff is greater than the weighted "Conscience" payoff, then $c>a$, consistent with the PD as well as Chicken. If the DM discounts profit compared to "Conscience", so that $a<c$, neither the PD nor Chicken can occur. Refraining may then be dominant. This inequality thus will not apply for Type IV DMs, would be more likely for Type II's than for Type III's, and would always apply for Type II's.

\section{An additional important inequality of Eq. (8.4): $a>b$}

The part of the inequality of Eq. (8.4) that may be the most interesting is the requirement that $a>b$ for both $\mathrm{PD}$ and Chicken:

$$
\begin{gathered}
C\left(x_{\text {few }}+N\left(x_{f e w}\right)>C\left(x_{\text {many }}\right)+N\left(x_{\text {many }}\right)\right. \\
N\left(x_{\text {few }}\right)-N\left(x_{\text {many }}\right)>C\left(x_{\text {many }}\right)-C\left(x_{\text {few }}\right)
\end{gathered}
$$

Unless a function that increases with $x$, such as $N(x)$, is included, neither the PD nor Chicken can develop because without such a function, it is impossible for $a>b$ : $C\left(x_{\text {fow }}\right)$ cannot be greater than $C\left(x_{\text {many }}\right)$ Recall that $N(x)$ represents the stake in the commons that players might have regardless of whether they Take or Refrain. There are four possibilities that allow $a>b$ : (1) players may already be Taking, so that the value of their current investments will be diminished by others Taking (Muhsam, 1977); (2) they may hope to Take sometime in the future; (3) they may place a higher current and future nonconsumable value on a commons in good condition than one in bad 
condition; or (4) they may feel foolish for Refraining if others Take (but not if others Refrain). Feeling foolish is not represented by any of these three attributes, but it could be included if the game were shown as a regret matrix.

Hardin's description of his herdsmen implied that they cared only for profit, and not at all about any nonconsumable value of the commons. Without $N(x)$, it is impossible for $a>b$ for any DM. Does this mean they could not be playing the PD? The answer to that question is fundamental to the difference between game theory and decision theory.

Hardin framed the herdsman's choice as whether or not he should add a cow to his herd, thus implying some stake in the commons even if the herdsman chose to Refrain. H.V. Muhsam (1977) argued that the TOC is algebraically a PD when the herdsmen already have herds grazing on the commons. The herdsman's existing herd will be negatively impacted if others add cattle, no matter what he does. This significant detail does not appear in an EMAV decision tree because it is independent of choice. If this decision is posed in the prescriptive decision theoretical way (as it is in the TOC simulations), then only the marginal attributes $\pi(T)$ and $C(x)$ should be included. Including $N(x)$ does no harm, because it has no effect; but it does make the calculations more complicated, without adding anything to the efficacy of the decision outcome.

However, if only marginal attributes are carried over into the game theoretical representation, then $N(x)$ would not be included there either. Games of Benevolent Chicken and Hero could then develop, but never PD or Chicken. If the DM's total 
welfare is represented, however, then $N(x)$ would be included in the payoff calculations. Representing the decision "at the margin," as an economist might say, leads to a different game theoretical analysis than representing non-marginal aspects of the decision. "At the margin," the dilemma of the PD is invisible.

A final requirement for the inequality of Eq. (8.9) to hold is that the slope $n$ of the $N(x)$ function must be steeper than the slope $m$ of the "Conscience" function; i.e., dividing both sides of Eq. (8.9) by $\left(x_{2}-x_{1}\right)$ and taking the limit as $\Delta x$ approaches zero yields:

(8.10) $N\left(x_{R}\right)-N\left(x_{T}\right)>C\left(x_{T}\right)-C\left(x_{R}\right)$

$$
\begin{aligned}
& \frac{d N}{d x}>-\frac{d C}{d x} \\
& n>-m
\end{aligned}
$$

It is thus necessary (though not sufficient) that the slope for the $N(x)$ function be steeper than the slope for $C(x)$, for Chicken or PD to develop. If Eq. (8.10) is reversed, the less dangerous games of Hero or Benevolent Chicken are more likely (as long as the other inequalities of Eq. (8.4) apply). What this means is that Chicken and the PD can only arise when (1) players receive a payoff that increases with $x$ (such as $N(x)$ ) even if they Refrain, and (2) players' nonconsumable valuations of the commons are more sensitive to changes in the commons than their "Consciences" are.

Intuition might suggest that it would make no sense for a person to actually prefer that others Take $(b>a)$, regardless of one's own actions. However, that is why 
Benevolent Chicken and Hero are such interesting games: in Hero, the player does not get to be a hero umless other people do the "wrong" thing (i.e., let the drowning victim drown). In Hero, players prefer that others Take so that they can get the glory of Refraining. This is analogous, for example, to a type of environmental activist who enjoys the spotlight and the "glory" of being more righteous than others, and would be disappointed if others actually did what the activist told them they should do (Dowie, 1995). In Benevolent Chicken, players are more generous or community-minded than in Chicken (or the PD), because they prefer that at least some people get their first choice.

\section{Conclusion}

A long-term solution to the TOC requires that enough players prefer to Refrain that the commons does not get destroyed. If players have a dominant strategy, it does not matter what decision rule they follow or what they think others will do: Type I's always Take, and Type IV's always Refrain. For the less hard-line DM Types, the choice is more problematic.

Types II and III Take when $N(x)$ is included and the commons is not in too bad condition, but they do not have a dominant strategy when either $N(x)$ is left out, or the commons is in bad condition (see Figure 8.10). If $N(x)$ is left out, Types II and III payoff graphs look like Benevolent Chicken or Hero. If $N(x)$ is included, Type II payoff graphs look like the PD when the commons is in good condition, but like Chicken if the 
commons is in bad condition. With $N(x)$, Type III graphs look like Chicken, Benevolent Chicken or Hero. Recall that Type III's are more like environmentalists than Type II's.

\begin{tabular}{c|l|l|}
\multicolumn{1}{c}{} & \multicolumn{1}{c}{$\boldsymbol{x}=\operatorname{good}$} & \multicolumn{1}{c|}{$\boldsymbol{x}=\mathbf{b a d}$} \\
\cline { 2 - 3 } $\begin{array}{c}\text { Type II } \\
\text { Type III }\end{array}$ & PD & Chicken \\
$N(x)$ not included & BC & Hero, BC, Clicken \\
\cline { 2 - 3 }$N$ & BC & Hero \\
\cline { 2 - 3 } & &
\end{tabular}

Figure 8.10. Summary of payoff graph game types, for Type II and III DMs. "BC" indicates Benevolent Chicken.

When these DMs do not have a dominant choice, their payoff graphs look like the N-person Chicken, Benevolent Chicken, or Hero graphs, and their preferences are then to do the opposite of what they think others will do. If they have some reason to believe that most other players' payoff graphs favor Taking, then they will Refrain because they expect others to Take. If they believe that other players' graphs are like Chicken, Benevolent Chicken, or Hero, then they have to estimate the condition of the commons and/or pick a decision rule. For example, if they use an expected value rule, pessimists will Refrain and optimists will Take. If they use a maximin rule, they will act like pessimists in that they will Refrain because it is the most conservative choice.

Thus, a solution to the TOC requires a healthy contingent of pessimists because pessimistic players with no dominant choice prefer Refraining. This suggests that increasing the sense of fear or caution could help solve the TOC; this is consistent with the approach of educating people about the disasters looming if humans do not change 
their ways. Interestingly, however, increasing people's appreciation for the nonconsumable value of the commons can only influence their preferences regarding other people's actions, because each person's own action has negligible effect on their own utilization of the nonconsumable commons value. That is the essence of commons dilemmas, of course: an individual's actions do not seem to matter; it is the actions of everyone together that matters. The fact that concern about what other people do is often what drives lobbying for governmental action is consistent with Hardin's argument that mutual coercion, mutually agreed on is the only feasible approach to solving social dilemmas (Hardin, 1968), although he was referring specifically to how to achieve population control.

The game theory analysis indicates that is not just pessimists that are required, but diversity in general, to solve the TOC. If everyone has the same payoff graphs, and follows the same decision rule, then all will either Refrain or Take. The only exception to this claim is that if everyone is a Type IV, then all will Refrain, which could be considered an extreme case of a solution to the TOC. For Type IV's, doing without consumable commons products such as lumber, beef, or fish presents no problem. However, although no definition of a TOC solution was found in the literature review, it seems reasonable to assume that a solution implies some kind of optimal utilization of the commons, and not just abandoning any kind of extractive activities altogether.

In contrast, if everyone is a Type I, then all will Take, and if they have no use for any kind of $N(x)$, again the outcome is theoretically acceptable to them; but it is not 
really a solution to the TOC because the commons would eventually be destroyed if the population grew. If instead the population is all Type II's, and everyone has a payoff graph like Chicken, and follows, say, a maximin rule, then all will Refrain, which is not a pareto-optimal outcome. Similarly, if everyone has a graph like Hero, is pessimistic, and follows an expected value rule, then all will also Refrain, and all will receive their next-to-worst outcome. And so on: conformity leads to ruin.

Unfortunately, even if there is enough diversity and sense of caution for a particular population size, the solution still will not be stable if population grows: once the maximum sustainable number of allowable Takets is reached, the population increase must consist primarily of cautious pessimists who prefer to Refrain. If the population grows, the profit function increases in magnitude, and so Refraining becomes less appealing as population, and thus demand, grows.

These major conclusions from the game theory analysis (that diversity is important and that population growth threatens the stability of any solution) were also important findings from the EMAV-based simulation experiments of the last section. Hardin said nothing about population diversity, but his thesis about the futility of any solutions that do not include population control appears to be robust from both a game theory and a decision theory perspective. It is interesting that Hardin's formulation does not produce a PD-like game unless it is assumed that the herdsmen have a stake in the commons even if they choose to Refrain. Related to this is the implication that an attribute $N(x)$ that is pivotal to the game theory results, is irrelevant to an EMAV 
analysis".

The importance of $C(x)$ in the EMAV simulations and game theory analysis conflicts to some extent with Hardin's dismissal of solutions based on "appeals to conscience." In the game theory analysis and the EMAV simulations, "Conscience" is an important factor in diminishing the inevitability of Taking. "Conscience" helps payoff graphs to conform to more tractable preference structures consistent with Hero or Benevolent Chicken, rather than more threatening preference structures consistent with the PD. A factor that game theory highlights, but is hidden in the EMAV simulations, is the role of beliefs about the state of the commons: the probability estimates in the EMAV decision trees that reflected DMs' beliefs labout the state of the commons were not found to be significant in any of the factorial experiments, implying that expectations about the state of the commons or number of Takers would not affect the dominance of a choice ${ }^{12}$. Yet the game theory analysis showed how expectations about the state of the commons could turn a game of PD into a game of Chicken, at least for the Type II DMs. Because PD has a dominant strategy whereas Chicken does not, this is an important finding that the EMAV analysis missed.

\footnotetext{
"Although $N(x)$ drops out of the decision trees, if a DMincludes $N(x)$, then $N(x)$ contributes to the "fitness" calculation used in the GA. This effect is examined in the solution simulations of the next chapter.

${ }^{12}$ As noted before, this was because of the rigidity of most of the DM Types, in that only the Type III's are likely to change their choices when expectations change. The optimism/pessimism alleles for the $g_{0}$ gene do sometimes turn out to be important, in the solution simulations.
} 


\section{SOLUTION SIMULATIONS}

Six overlapping categories of solutions to the TOC were discussed in Chapter IV: technology, community identification and moral considerations, financial incentives, property rights, regulatory control, and population control. A seventh possibility, nonconsumable value $N(x)$, was found to be important by the game theory analysis. In this third, and final, phase of the work, the results of the factorial experiments from Phase II (Chapter VII) were used to select a baseline simulation, which was then modified in order to simulate important characteristics of these potential solutions. A summary of the solution categories, how they were simulated, and a brief explanation for each, is given in Table VII.

Solutions based on privatizing the commons cannot be represented with the simulation, because the simulation is based on an assumption/ of common access; in effect, the private property solution to the TOC means eliminating the commons, which is not assumed to be a solution. Population control is included in the analysis of each solution approach rather than explicitly as a separate category, because the question investigated was whether any of the solutions could be made to work without population control. Also, it was assumed and demonstrated in Phase I that population control could solve the TOC (see Figures 7.1 and 7.3), because as long as the number of Takers is kept below the sustainable harvest level (100 commons units per iteration), 


\begin{tabular}{|c|c|c|}
\hline Solution eategoriy & $\begin{array}{l}\text { Parameter(s) or functions } \\
\text { used to simulate }\end{array}$ & Explanation \\
\hline \multirow[t]{2}{*}{ 1. Technolagy | } & 1a. Profit function parameter $k$ & $\begin{array}{l}\text { Technology decreases } \\
\text { demand for the commons by } \\
\text { providing substitutes and/or } \\
\text { increasing efficiency. }\end{array}$ \\
\hline & $\begin{array}{l}\text { lb. Commons regeneration rate } \\
\text { constant } r_{0}\end{array}$ & $\begin{array}{l}\text { Technology increases supply } \\
\text { by increasing the } \\
\text { regeneration rate. }\end{array}$ \\
\hline 2. Community idlentification & $\begin{array}{l}\text { "Conscience" function } \\
\text { parameter } m\end{array}$ & $\begin{array}{l}\text { Responsibility to others is } \\
\text { represented by increasing } \\
\text { the sense of guilt for Taking } \\
\text { and/or virtue for Refraining. }\end{array}$ \\
\hline $\begin{array}{l}\text { 3. Noncorisumablle value } \\
\text { function }\end{array}$ & $\begin{array}{l}\text { Nonconsumable attribute } \\
\text { function } N(x)\end{array}$ & $\begin{array}{l}N(x) \text { drops out of decision } \\
\text { trees but was shown to be } \\
\text { potentially important in the } \\
\text { game theory analysis. }\end{array}$ \\
\hline 4. Financial incelntives & $\begin{array}{l}\text { Penalties or bonuses modifying } \\
\text { the profit function } \pi(T)\end{array}$ & $\begin{array}{l}\text { Taxes, credits, and fines are } \\
\text { used to reward potential } \\
\text { Takers for Refraining or } \\
\text { penalize them for Taking. }\end{array}$ \\
\hline 5. Property rights & Not simulated & $\begin{array}{l}\text { If the commons is } \\
\text { liquidated, or otherwise } \\
\text { divided up among users, the } \\
\text { TOC no longer applies and } \\
\text { the simulation does not well } \\
\text { represent the situation. }\end{array}$ \\
\hline 6. Regulatory control & $\begin{array}{l}\text { Penalty function for Taking if } \\
\text { more than the harvest limit } \\
\text { MaxTakers is cxceeded }\end{array}$ & $\begin{array}{l}\text { A maximum allowable } \\
\text { harvest level MaxTakers is } \\
\text { enforced with a penalty } \\
\text { function imposed on illegal } \\
\text { Takers who get caught. }\end{array}$ \\
\hline 7. Populatian control & $\begin{array}{l}\text { Exponential population growth } \\
\text { parameter } G \text {, upper limit to } \\
\text { population size Pop } p_{\max } \text {, and/or } \\
\text { profit function parameter } k \text {, } \\
\text { "Conscience" function } \\
\text { parameter } m \text {, regeneration rate } \\
\text { constant } r_{0}, \text { and } \\
\text { nonconsumable attribute } \\
\text { function } N(x)\end{array}$ & $\begin{array}{l}\text { Included in each solution } \\
\text { analysis rather than as a } \\
\text { separate section, either by } \\
\text { controlling population, or by } \\
\text { demonstrating how any } \\
\text { solution has to change if } \\
\text { population is allowed to } \\
\text { grow. }\end{array}$ \\
\hline
\end{tabular}

Table VII. Solution categories, methods for simulating, and brief explanations. 
the commons is assumed to be able to regenerate. Thus, this chapter consists of simulations to explore the technology, community identification, financial incentives, nonconsumable value, and regulatory control approaches to solving the TOC. Each approach is examined in the light of the effects of population growth.

Unless otherwise indicated, "Conscience" is included in all the simulations, because it is difficult to imagine that any real village could not have some people who take some degree of community or moral considerations into account. With the "Conscience" function at a fairly low level $(m=0.02)$, and profit at a fairly high level $(k$ $=3.0$ ), "Conscience" has enough of an effect to allow a population of up to about 150 potential Takers to harvest at or below the sustainable level (100 units) indefinitely. If the population grows, demand increases to the point that the effects from "Conscience" are overwhelmed. Then, unless some other solution mechanism is implemented, the commons will be destroyed.

\section{Technological solutions}

One of the abiding hopes of many people in the world is that scientists and engineers can find ways to mitigate if not solve the growing problems with commonses in the world. Fish farming, for example, now provides $14 \%$ of the fish sold in the world, and the percentage is increasing rapidly (Brown, Lenssen, and Kane, 1995). Most of the wild salmon runs in the Columbia River basin have been replaced with declining populations of hatchery fish (Huntington, Nehlsen, and Bowers, 1996). Air 
pollution has been decreased in many places by automobile exhaust cleaning technology such as catalytic converters and increased efficiency of internal combustion engines, and by "scrubbers" installed in factory exhaust pipes (Brown, Lenssen, and Kane, 1995). Sewage treatment plants remove much of the pollution that cities would otherwise pump into rivers. And so on.

Technology is often viewed not as the hero but as the villain, however. For example, in Atlantic as well as Pacific ocean fisheries, international fleets of huge factory trawler ships using sophisticated technologies for finding and catching fish have decimated the once-abundant cod, mackerel, herring, anchovy, haddock, halibut, and other stocks that were sustainably fished for centuries by srnall-scale artisanal fishers. As the stocks declined, technology provided ever-more-effective tools for finding and catching the smaller, younger, and more widely dispersed fish that remained as the fisheries declined. The fish populations collapsed, and trawlers switched to other species. Artisanal fishers, out-competed and out-maneuvered by the international fleets, appealed to their governments for help, and many went out of business (Fairlie, Hagler, and O'Riordan, 1995). None of the decimated stocks have ever recovered.

Whether the net effects of technology are beneficial or harmful is not the question here, however. The question is whether it makes sense to assume that technology can make it possible for the human population to continue growing and consuming at the current rate: can the TOC be averted by technology?

These simulations investigate, in a very simple way, what might be required for 
technology to solve the TOC, given a growing population. The simulations rely on "best case" assumptions about human rationality and the regeneration of the commons. The baseline simulation (see Figure 8.11) is essentially the second simulation of Phase 1, where potential fishers trade off profit and "Conscience" in making their decisions. In this baseline case, as long as the number of potential Takers stays below about 150, the village harvests sustainably enough to allow the commons to regenerate itself. If the population grows, however, demand increases, and the harvest level exceeds the ability of the commons to regenerate (Figure 8.11) ${ }^{13}$. Then the commons is destroyed.

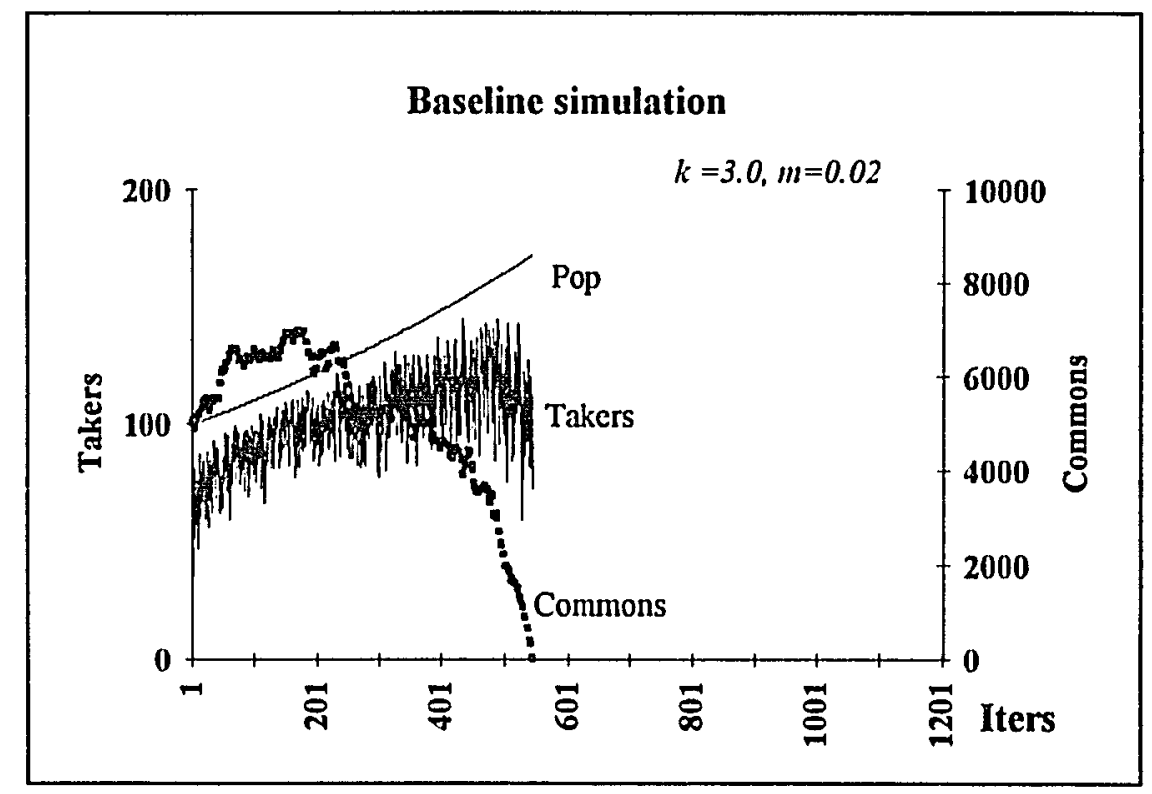

Figure 8.11. TOC with population growth $G$ at 0.001 per iteration, profit function paramter $k$ of 3.0 , and "Conscience" function parameter $m=0.02$. The same simulation with $G$ of 0 produces sustainable harvesting forever, but with a growth rate of $0.1 \%$ per iteration, the commons is destroyed by about 550 iterations.

\footnotetext{
${ }^{13}$ The graphs shown in this section were chosen because they were typical of the results for multiple runs using different random number seeds.
} 
Technology can theoretically help in three ways: 1) increasing the effective regeneration rate of the commons via, for example, hatcheries; 2) providing substitutes via, for example, fish farms or providing alternate food sources; and 3) increasing the efficiency of use, so that one fish, for example, feeds more people. The later two approaches (providing substitutes and increasing efficiency) work to decrease demand, which is represented in the code by the marginal profit function parameter $k$. The first approach (increasing supply) can be represented in the simulations by increasing the effective regeneration rate constant $r_{0}$.

\section{Decreasing demand}

It was shown in the factorial experiments of Phase II that decreasing $k$ is indeed an important factor in allowing the commons to survive. However, as long as demand otherwise tends to increase with population, technology must work to decrease $k$ at least as much as population tends to increase it. For example, cutting $k$ in half in the simulation permits the commons to last another couple of hundred iterations, but eventually the population grows to the point that the commons is demolished (Figure 8.12).

In order for the population to continue growing without destroying the commons, $k$ would have to continue declining, due to improving either efficiency or the availability of attractive substitutes. To simulate this, the code was modified so that whenever the commons falls below some critical value, such as $x_{\max } / 4\left(x_{\max }\right.$ is the 
maximum level that the commons can be), $k$ is decreased by an arbitrarily chosen value of $10 \%$. The result is shown in Figure 8.13 .

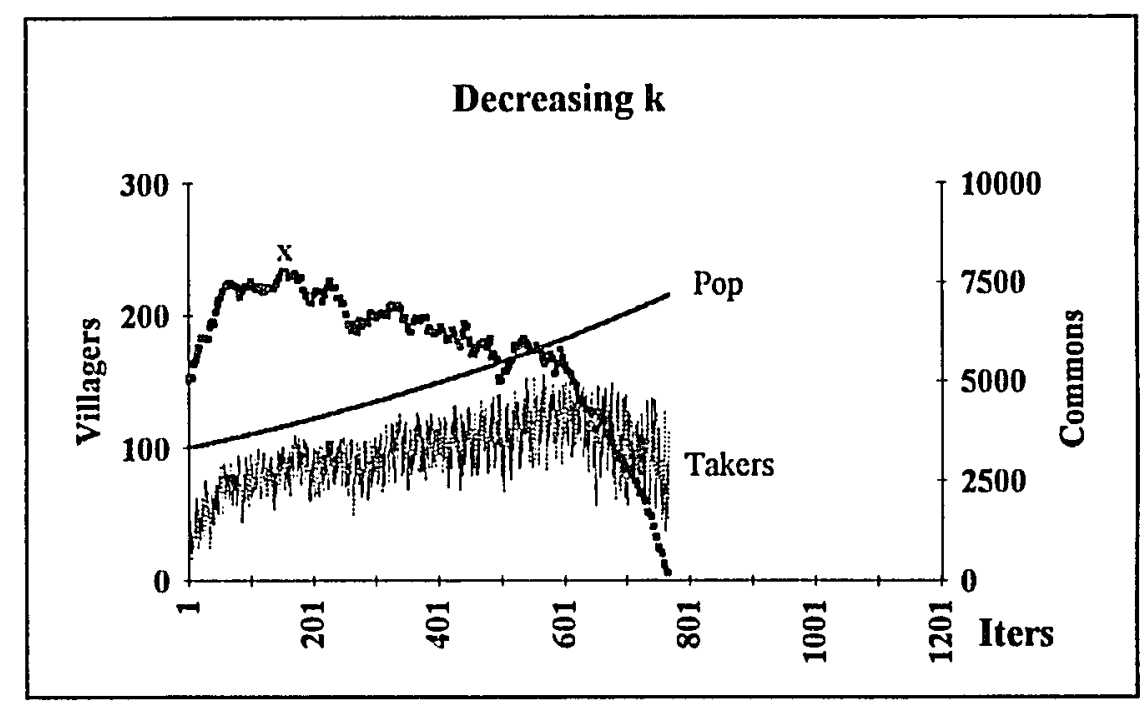

Figure 8.12. The baseline simulation (Figure 8.11), but with demand parameter $k$ decreased by half. The commons survives longer than before, but still is harvested to extinction.

The stepwise reduction in $k$ reflects a decrease in the slope and $y$-intercept of the marginal profit function ( $k$ is essentially the slope of the demand curve). Recall that in the base simulation, demand increases proportional to population. Technology's challenge is to counteract this growing demand by providing substitutes or efficiencies that decrease demand. In Figure 8.13, as population grows, the initial slope $k_{0}=3.0$ remains constant up to the point that the increasing number of Takers has depleted the commons down to $x_{\max } / 4$ (at about iteration 98 ). Then technology decreases $k$ by $10 \%$, but $x$ is still below $x_{\text {max }} / 4$ and so $k$ is immediately decreased again, and again - until 
$x$ gets the chance to climb back above $x_{\max } / 4(k=0.9) . k$ remains constant at the new value while the population continues to grow, until $x$ drops too far again, and so on until the end of this run, when $k_{f}$, the final value for $k$, drops to 0.3 .
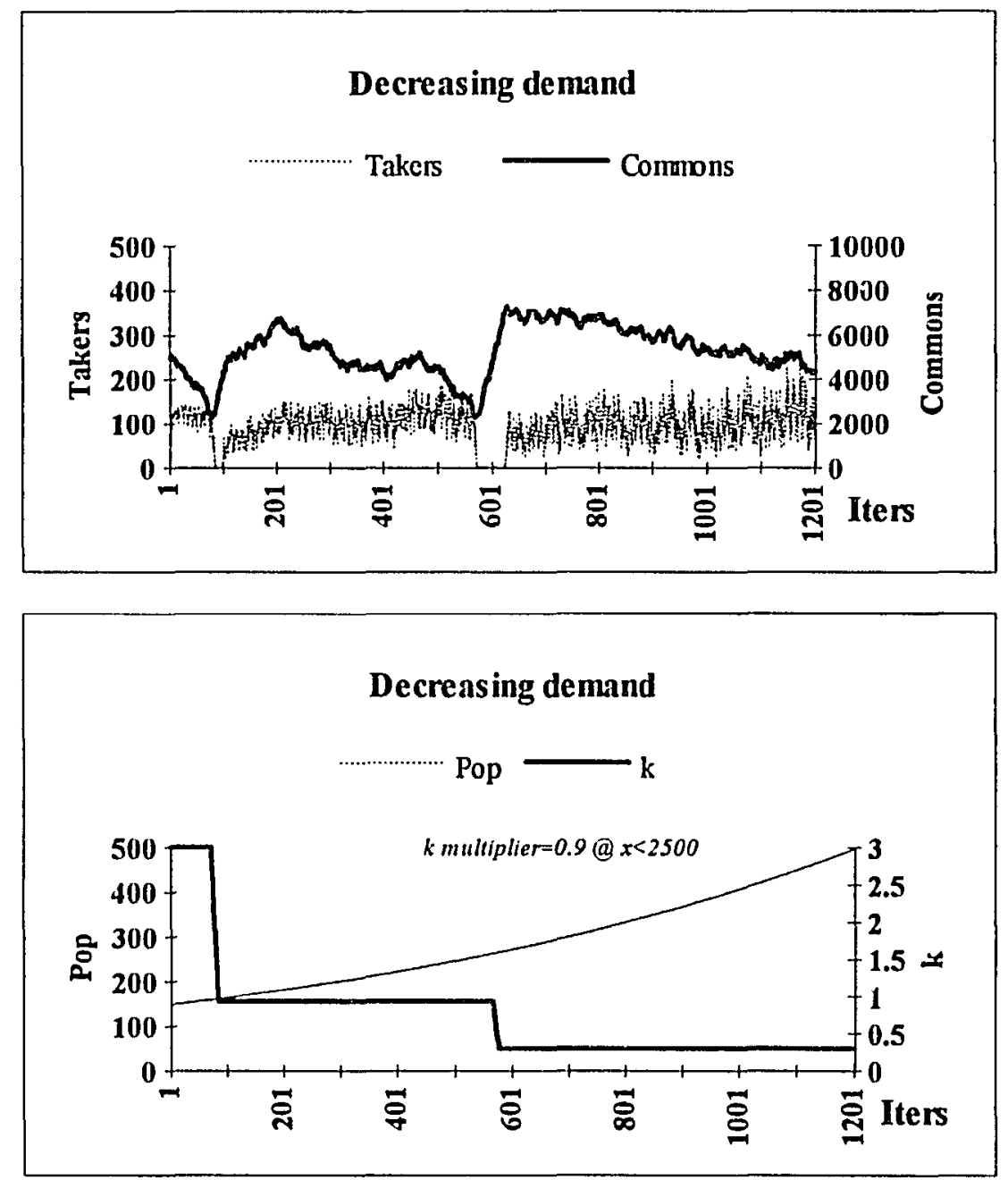

Figure 8.13. Simulation results when the profit function parameter $k$ is decreased by $10 \%$ if the commons falls below $x_{\max } / 4$. The upper graph shows the number of Takers and the state of the commons over 1200 iterations; the lower graph shows the total population and $k$ over the same period. 
Demand is thus torn between two forces: growing population driving it up, and technology driving it down. At the beginning of the simulation in Figure 8.13, the ratio of the profit parameter and the "Conscience" parameter is $k / m=150$; but by the end of the simulation, the ratio is only 14.8 . "Conscience" is then relatively much more important compared to profit, simply because demand for the commons product has dropped dramatically, while no change has been made in the "Conscience" function.

In order for the commons to survive in this simulation, technology has to continually increase the availability of attractive substitutes, or increase efficiency of use. In order to sustain about a tripling of population, technology has to decrease demand by a factor of ten $\left(k_{0} / k_{f}=3.0 / 0.3\right)$. To allow population to continue growing, it would be necessary for technology to continue decreasing demand, at an exponential rate, at the same time that there are exponential increases in demand due to growing population. Harrison points out that it is not only growing population but also growing per-person consumption that creates the challenge for technology today. He says that even using the U.N.'s lowest projections, by 2050, per-person consumption of natural resources will have doubled. In order to maintain today's destructive rate of impact on the environment, let alone to decrease it, Harrison says that technology would have to cut the damage done by each unit of consumption by 72 percent (Harrison, 1994).

Unfortunately, there is good evidence that the law of declining marginal returns, and not the law of exponential growth, tends to apply to technological progress with 
respect to natural resources. For example, the technological wonders that created the "green revolution" in agriculture initially developed at an exponential rate. The green revolution at first caused food prices to drop and supplies to increase, not only because of increased efficiency but also because of the ongoing introduction of new and improved products. In recent years, however, the rate of increase has fallen off in a way that looks very much like a typical graph of a function exhibiting declining marginal returns (Brown, Lenssen, and Kane, 1995). The decline is attributed primarily to increasing degradation of commonses world wide: growing populations have replaced former farmland with cities, grazing areas have become severely degraded due to overgrazing and desertification, groundwater supplies have become diminished, and the biodiversity of important bacteriological and insect "friends" has declined, while the pesticide-resistence of bacteriological and insect "foes" has increased. Technology, which often has actually helped exacerbate these relatively new problems, has had little success solving them.

Scientists have also had little success developing substitutes for any vital commonses. This is a particular concern in poor countries. For example, much of the "third world" depends on local fisheries for most of their protein, and can neither turn to increased imports (because of relative poverty) nor to new local food sources such as farming, since environmental degradation tends to be particularly acute in these areas. In ironic contrast to the difficulties poor countries have in finding substitutes for the fish on which they are so dependent, rich countries have actually increased their 
rate of substitution of fish for other meats: in 1988-1989, consumers in rich countries ate nearly three times as much fish per person as did people in poor countries (Kent, 1995). Not only do we in the north eat more fish (much of which comes from poor countries), we use more fish to feed the poultry and pork that we are increasingly substituting for beef: $30 \%$ of the world's fish catch is converted to fishmeal, and half of that is exported to developed nations to feed poultry and pigs (Kent, 1995). Technology is certainly playing a role in this conversion, but so far it has hurt, not helped, the destruction of the world's fishing commonses.

\section{Increasing supply}

If cutting demand is not enough, then perhaps increasing supply will work. For example, technology could increase the regeneration rate constant $r_{0}$ when a commons becomes depleted to some level, say $x_{\max } / 4$. In the simulation shown in Figure 8.14, the regeneration rate increases by $20 \%$ whenever the commons falls below $x_{\max } / 4$.

Technology saves the day. However, $r_{0}$ has to increase tenfold - from 0.04 to $0.4-$ in order to sustain a little more than a threefold population increase. Since population increases exponentially, $r_{0}$ has to increase exponentially as well. The increase in $r_{0}$ is guaranteed in the simulation, as is the determination of the critical commons level at which the increase is needed. This is not the case in the real world. Furthermore, the real world is stochastic. Stochasticity of the commons was held low in the above examples (Stoch $=0.8$, but increasing it slightly (to 1.0 ) leads to the results shown in Figure 8.15. 

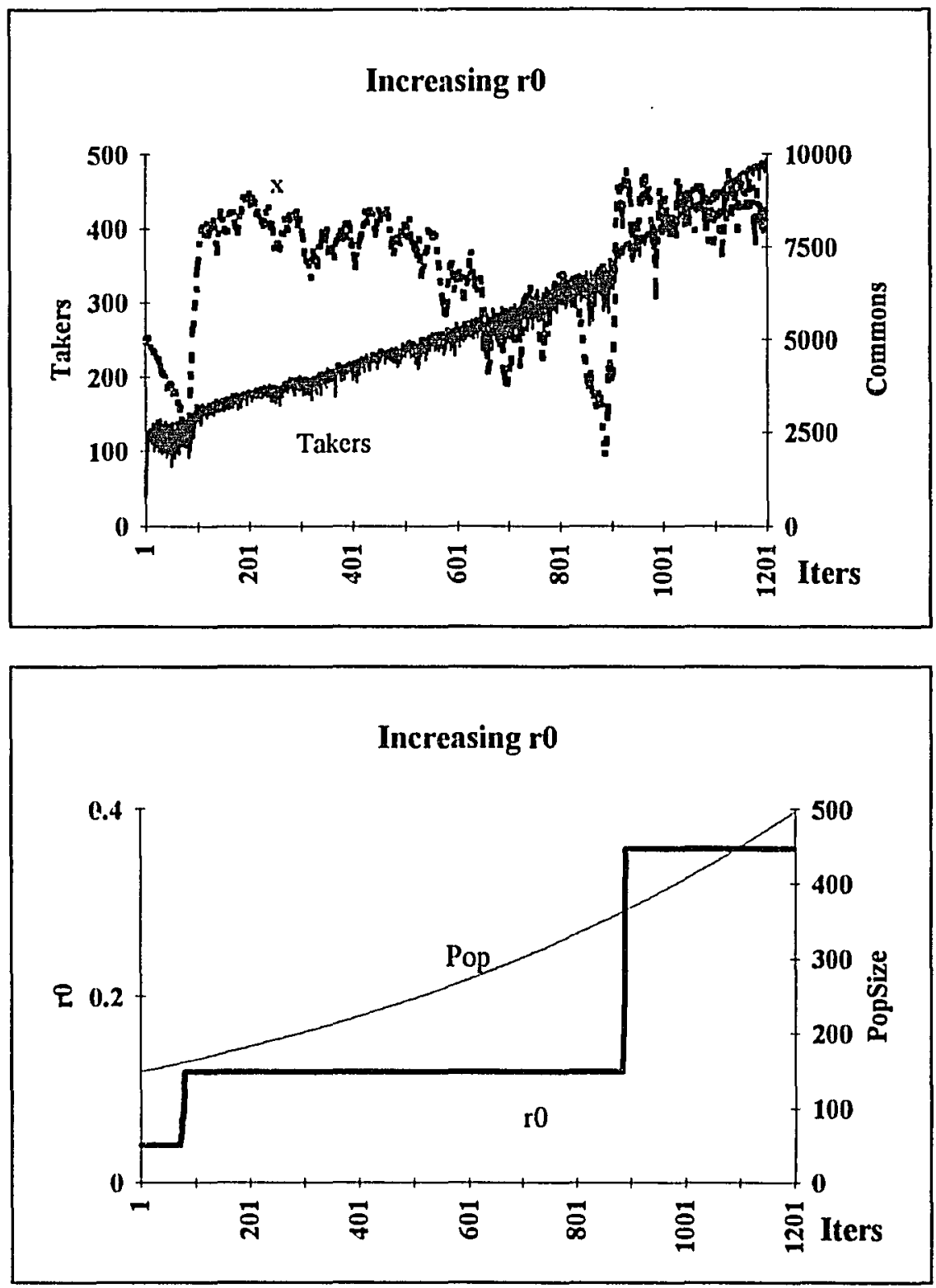

Figure 8.14. Baseline simulation (Figure 8.11), but with a $20 \%$ increase in $r_{0}$ whenever the commons falls below $x_{\max } / 4$. The upper graph shows the number of Takers and the state of the commons over 1200 iterations, while the population grows from 150 to 500 . The lower graph shows $r_{0}$ and the total population over the same period. 

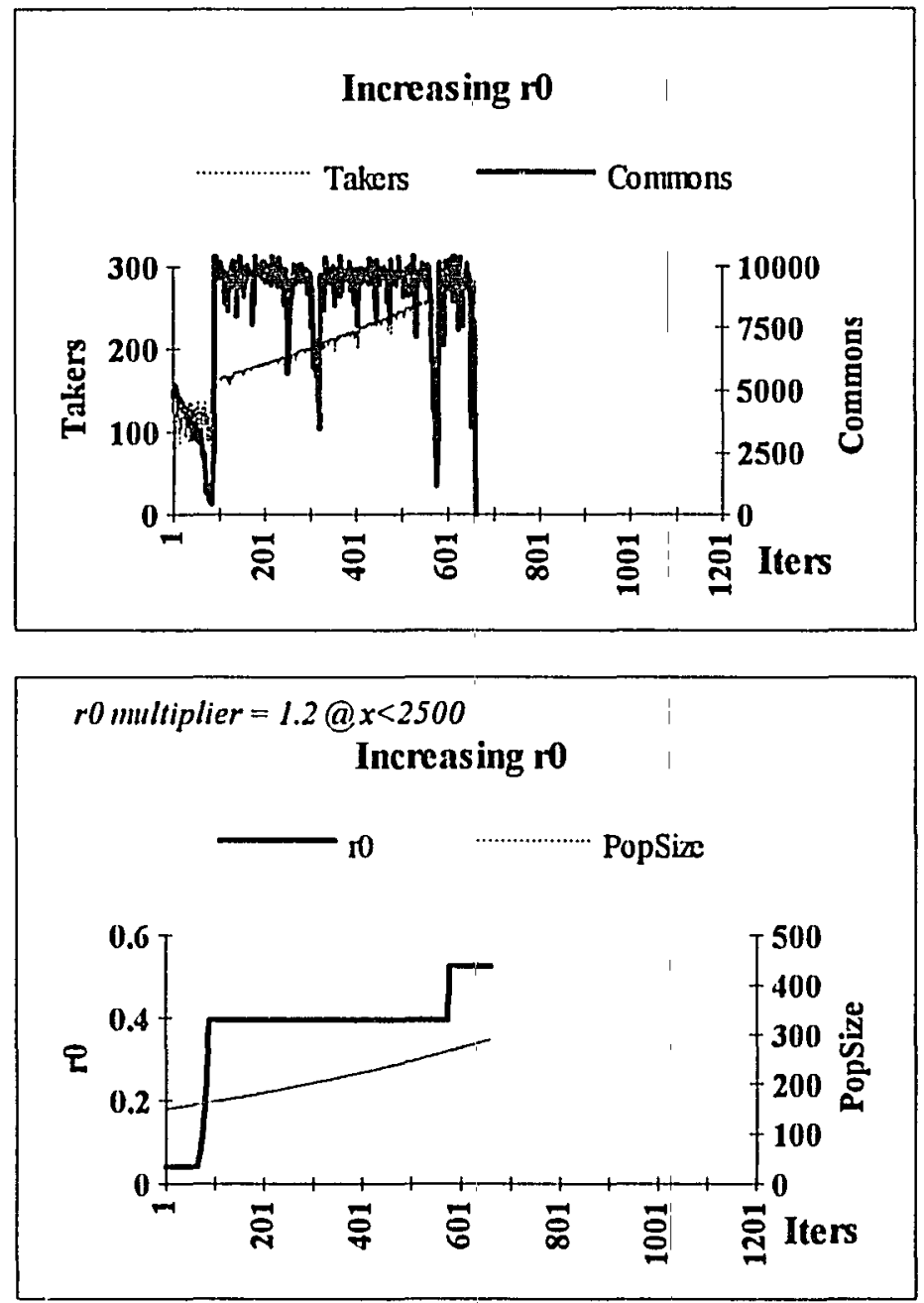

Figure 8.15. Baseline simulation (Figure 8.14), but with a more stochastic commons (Stoch $=1.0$, compared to 0.8 in previous examples). Increasing $r_{0}$ by $20 \%$ is adequate to save the commons with relatively low stochasticity, but not when it is increased slightly.

Hardin's (1968) main thesis was that population growth belongs to the class of problems for which there is no technological solution. The simulations suggest that if supply could be reliably increased and/or demand reliably decreased, technology can 
help solve the TOC. However, for population to continue growing exponentially indefinitely, technology must also grow exponentially indefinitely, with some margin to allow for stochasticity. That is, engineers and scientists must find new substitutes, increase efficiency, and/or increase commons production, at an exponentially increasing rate.

If this seems reasonable to expect, then Hardin's thesis would not be supported. However, the evidence of the "green revolution," let alone the world's fisheries, is not encouraging. Technology's response to depleted ocean fisheries has been to fish more efficiently and voraciously, thus accelerating, not mitigating, the destruction. In agriculture, the initial miraculous efficiency improvements and new product developments of the green revolution have more recently exhibited a clear/dependence on the law of declining marginal returns. If the law of declining marginal returns and not the law of exponential growth applies to increasing the supply of, or decreasing the demand for, natural resources, then Hardin's thesis is supported.

\section{Community identification and moral concerns}

Commons management by small, close-knit communities has a long and mostly successful history, at least until the current century. In the simulations, the "Conscience" function $C(x)$ is used to represent the major driving force behind successful community-based management, as well as the effects of (other) moral considerations. 
"Conscience" adds a penalty for Taking and/or a reward for Refraining to some of the DMs' decision trees and game matrices. The simulation outcome for a relatively low "Conscience" parameter $m$ is shown above in the baseline simulation of Figure 8.11: the profit parameter $k$ is 3.0 and the "Conscience" parameter $m$ is $0.02 . \mathrm{k} / \mathrm{m}=$ 150 , and "Conscience" is inadequate to prevent the destruction of the commons. Doubling $m$ permits the commons to survive up to a maximum population of about 200 (see Figure 7.3, Chapter VII). What would it take for $m$ to allow indefinite population growth?

To answer this question, a similar approach was taken to that used for decreasing $k$ or increasing $r_{0}$. In the computer code, $m$ is increased by an arbitrarily chosen $10 \%$ whenever the commons falls below $x_{\max } / 4$. Nothing else is changed: there are still the same four DM Types, each of which has a pessimist version and an optimist version, stochasticity is relatively low $($ Stoch $=0.8)$, and $k=3.0$. The result is shown in Figure 8.16.

The initial ratio $k / m$ in the simulation shown in Figure 8.16 is 3.0/0.02 or 150, and the final rat:o, after the population increases by a factor of 3.3 (to 500), is 15.2 . The conclusion that could be drawn here is that increasing the relative importance of DMs' "Consciences" whenever the commons becomes depleted does allow the commons to survive. Those who still decide to Take and are unaffected by their "Consciences" (Type I's ) still receive the same profit payoff, but when the "Conscience" function becomes relatively more important, those who are affected by 
their "Consciences" (Types II, III and IV) receive higher payoffs. The selection mechanism of the GA tends to help them prevail, and thus to save the commons.
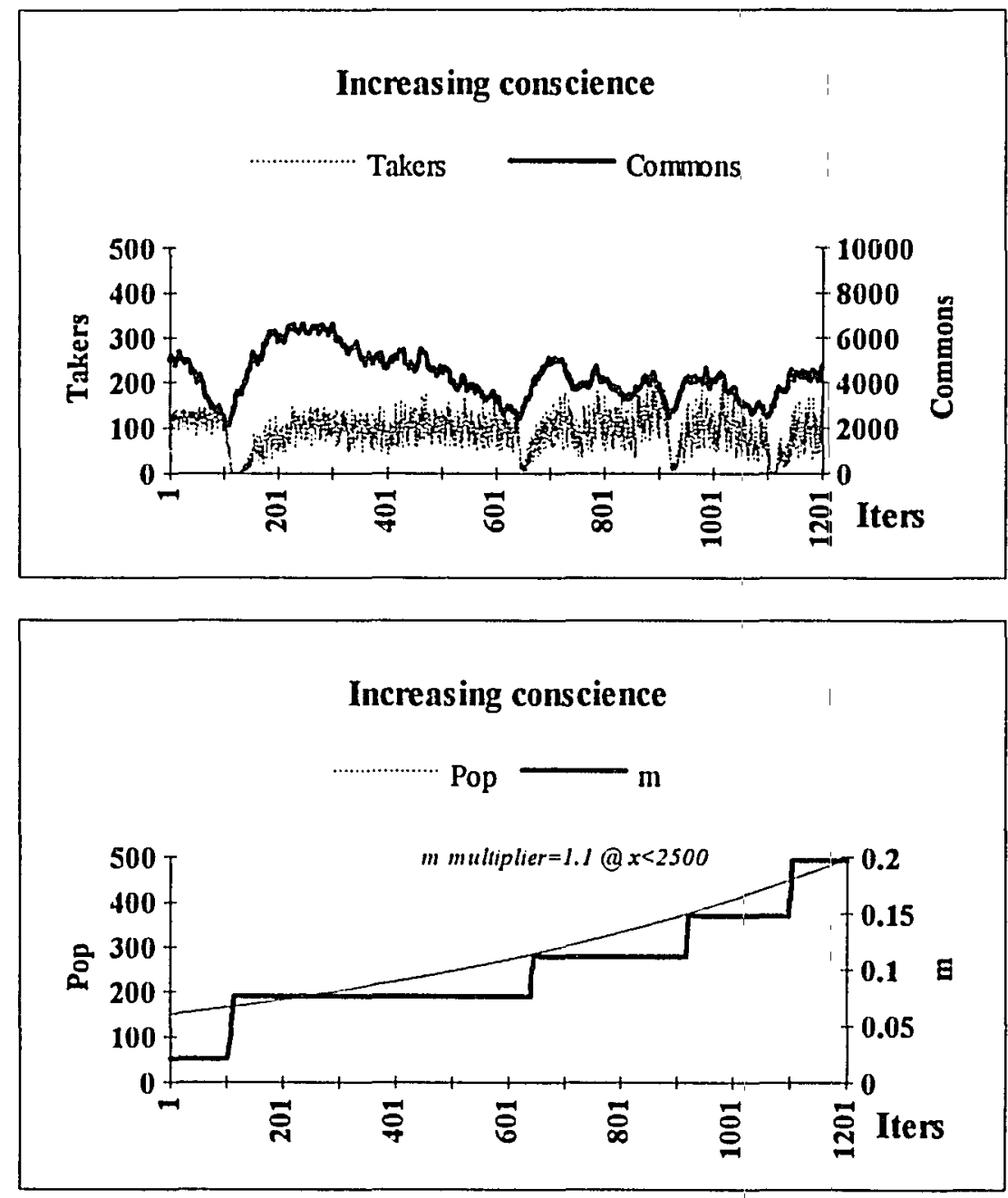

Figure 8.16. Simulation results for increasing "Conscience" function parameter $m$ by $10 \%$ whenever the commons falls below $x_{\max } / 4$. Population more than triples while $m$ increases tenfold.

What this might represent in the real world is increased power for the conservationist movement, as more people become concerned about the social and 
ethical implications of degrading a natural resource. Increasing $m$ represents decreased social approval of commercial activities that harm the commons, leading perhaps to the enactment of stronger environmental laws. A smaller portion of the population would be engaged in harvesting the commons, and would receive higher profits because of the drop in supply; but because of the decreased supply and higher prices, consumers would have to pay higher prices, do without, or switch to substitutes. It is difficult to imagine a growing population being able to simply give up an important food source or source of income unless something else becomes available; hence, for this solution to work, technology would probably also have to be developing substitutes so that people have jobs and resources to switch $t o$. Otherwise, decreased supply and higher prices would eventually counteract the effects of increased $m$, and the "Conscience" solution would fail.

For example, environmental laws such as the Endangered Species Act cancelled most public timber sales in the Pacific Northwest in the late 1980's, just when a population and building boom began in the region. Demand for lumber increased at the same time that supplies decreased. The outcry from the affected public eventually led to a suspension of environmental laws so that logging could resume. The same kind of thing has happened repeatedly during the past century in countless small towns dependent on fisheries all over the world: at the same time that supplies are decreasing, demand is increasing. In the simulations, as $x$ declines, the value of "Conscience" $C(x)$ increases stepwise - but if increasing population causes demand to increase, it will 
always eventually outstrip the effects of "Conscience," unless the $C(x)$ function itself increases, via increasing $m$.

This suggests a potential cyclic pattern to increases in demand and "Conscience". For example, having $m$ increase by $10 \%$ whenever the commons falls below, say, $x_{\max } / 4$, while demand is steadily increasing leads to a kind of accelerating arms race between the conservationists and the fishers. Whenever the commons becomes too depleted, conservationists raise the "Conscience" level, , and lobbying for stricter controls or laws. If they are successful, unemployment may increase, and consumers may begin to object to lower supplies and higher prices. Extractors and consumers increase demand to the point that potential profit again exceeds the impacts of "Conscience". And so on, as the cycle repeats.

It is hard to imagine this contest continuing indefinitely in the real world, as it could in the simulation. One side or the other could get tired of the battle. Extractors could stay in power long enough to destroy the commons, or conservationists could eventually cause extractors to give up and switch to another profession. Technology could provide substitutes or efficiency measures that reduce demand.

How long the contest continues depends on how long the commons survives: once the commons is gone, the contest is over. The extractors can end the battle any time by either giving up or by destroying the commons. If they give up, and the commons recovers, the contest could start all over again. The effect of the conservationists is actually to prolong the fight by conserving the commons. The only 
way they can end the contest is by giving up so that the extractors destroy the commons, or by somehow reducing demand to the point that extractors choose, or are forced, to give up themselves. In a game theory matrix, this contest might look like Figure 8.17.

\begin{tabular}{ll|l|} 
& \multicolumn{2}{c}{$\begin{array}{l}\text { Envirommentalists } \\
\text { Give up }\end{array}$} \\
& & Fight \\
Takers Refrain & 1,3 & 0,2 \\
\cline { 2 - 3 } Takers Take & 3,0 & 2,1 \\
\cline { 2 - 3 }
\end{tabular}

Figure 8.17. Game between conservationist and extractor pressure groups, showing ordinal payoffs where the maximum $=3$ and the minimum $=0$. Takers have a dominant strategy (always Take), whereas conservationists prefer to fight if the Takers Take ive up if Takers Refrain.

In the game of Figure 8.17, Takers will always Take, regardless of what the conservationists do. Conservationists do not have a dominant strategy, but prefer to fight if Takers Take give up if Takers Refrain. They know Takers have a dominant strategy, and so they must continue fighting: they end up in the lower right hand cell of the matrix in Figure 8.17. This is a stable outcome, as well as pareto optimal, at least in theory. In this formulation, the game goes on as long as the commons survives. For the game to change, the rules or payoffs would have to change as well.

When there was a rush to enact new environmental laws in the 1960 s and 1970s, the conservationists were winning. Takers did not give up, however, and by the mid-1990s, attacks on those laws by Congress implied that the Takers were winning for 
the moment, although conservationists had not given up either. If the relaxation in environmental laws leads to increased commons destruction, then the level of public alarm may rise again. Neither side has shown any inclination to give up, consistent with the payoffs shown in Figure 8.17.

It is worth considering briefly what the effects might be of different formulations for the "Conscience" function discussed in Chapter VI. Although $C(x)$ is modeled here as a linearly decreasing function of $x$, a case could be made for it being something different, particularly a higher order or exponential function, a nonmonotonic but still continuous "humped" function, or a step function.

For example, instead of increasing linearly as $x$ declines, $C(x)$ could increase at an increasing rate as the commons declines, in the form of a higher order or exponential function (see Figure 8.18).

Then, when the commons becomes severely endangered, $C(x)$ might be more effective at getting DMs to Refrain because its worth, relative to the profit function, would be greater than it is as an equivalent linear function. This would appear to enhance its potential for solving the TOC, because the impacts of an exponential $C(x)$ would be greater at lower $x$, when they are most needed, than they are for an equivalent linear $C(x)$. It does not seem unreasonable to believe that at least some people become much more alarmed about threatened commonses than a linear $C(x)$ implies, and for them an exponential $C(x)$ would appear to be more reasonable. 


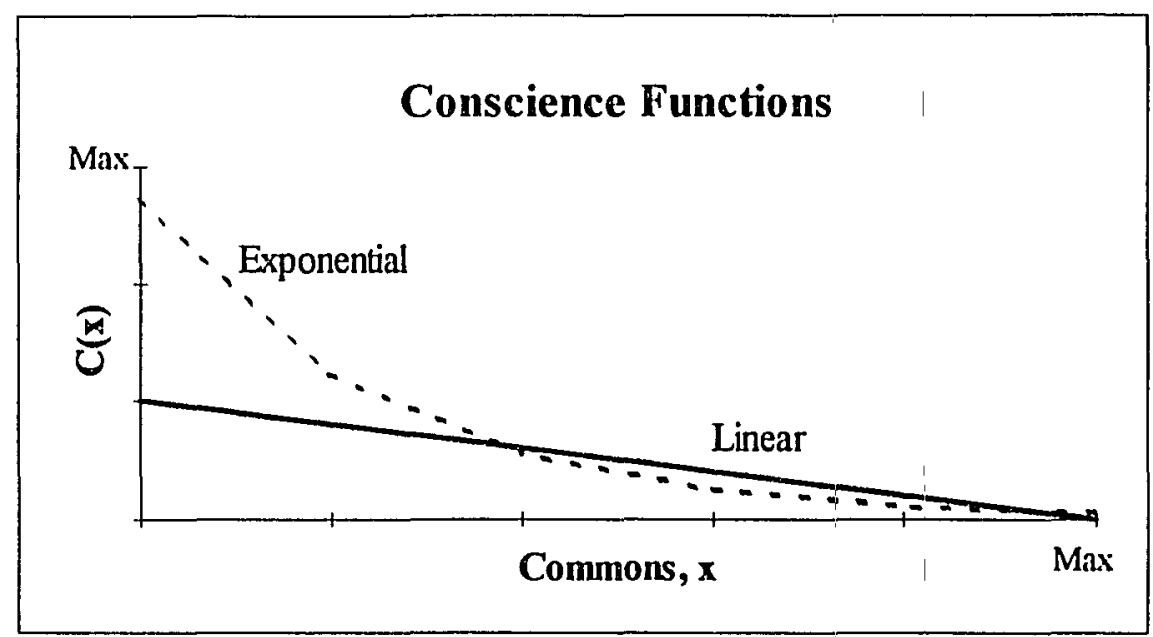

Figure 8.18. Examples of linear and potential exponential forms of the "Conscience" function $C(x)$.

There is not as much difference between an exponential $C(x)$ and an equivalent linear $C(x)$ as it might seem, because the exponential form is essentially equivalent in effect to a series of linear functions whose slopes increase as $x$ declines. In the simulations described above, a "Conscience" solution can always be found for any population size as long as the "Conscience" function parameter $m$ can increase as needed. The slope of $C(x)$ that really matters is the slope when $x$ drops low enough to be endangered, because as long as $x$ remains adequate, there is enough self-restraint in the population that the DMs can avoid destroying the commons.

There are three main difficulties that the exponential function approach has in common with the linear formulation: (1) Just as with the linear $C(x)$, as long as profit is proportional to population while $C(x)$ is a function of the commons, increased demand due to increased population will still eventually outstrip the effects of the "Conscience" 
function. At what point that occurs depends on the slope and $y$-intercept, regardless of whether the function is linear or exponential. (2) As species become threatened or endangered, their ability to recover is increasingly sensitive to environmental stochasticity. There is a threshold value for $x$ below which a species or population cannot recover, and any form of $C(x)$ would have to stop the harvesting before the commons falls below that threshold value, or the commons cannot recover. No one knows what that threshold value is for any species, and the problem is exacerbated because environmental and species demographic stochasticity increases as habitats become degraded. (3) Just as with the linear formulation, different people have different threshold alarm levels, and enough of them would have to have a high enough cutoff point to prevent commons destruction.

As for the "hump" shaped $C(x)$ discussed in Chapter VI, recall that for this kind of $C(x)$, the maximum values fall somewhere in the middle of the range of $x$ rather than towards the lower end. If the commons is in very poor condition, instead of caring more about saving the endangered commons, as they do in the linear or exponential formulations, DMs may decide that it does not matter what they do since the commons is doomed anyway. Then $C(x)$ approaches zero as $x$ declines. At the other end of the continuum, just as with the linear or exponential formulations, most DMs believe that as long as the commons is in excellent condition, again it does not matter what they do. Thus, DMs with a "humped" $C(x)$ would be more reluctant to Take while $x$ was in the mid range but less reluctant to Take if the commons became degraded (or if it was in 
good condition). Particularly if stochasticity is a factor, such a "Conscience" function would not tend to help solve the TOC because its effects would become less effective just as the need for it intensified. Although no rigorous study was conducted, during the development stages of this work, simulations were performed with the linear $C(x)$ replaced with various "humped" functions, and no discernible difference in outcome was found.

A third possibility is to represent the "Conscience" function as a stepwise, or discontinuous, function. The Endangered Species Act, for example, "kicks in" at a very low level of $x$ and otherwise requires no particular activities to protect species that may be at risk but are not defined as "threatened" under the Act. If the steps increased uniformly as $x$ declined, it would have no discernible effect on the ultimate conclusions because the effect would be approximately the same as a linear function drawn through the steps. If they increased more steeply, the equivalent continuous function would be polynomial or exponential, with the effect discussed above; and if a more "humped" function could be fitted through the steps, the effect would be roughly the same as for the continuous "hump" function.

As discussed in Chapter VI, it is difficult to say what people's various "Conscience" functions ought to be, and the linear form was chosen/because it seemed reasonable and because there was no compelling reason for anything more complex. Nonetheless, this could be a fruitful line for further research. 


\section{Nonconsumable value}

In the game theory analysis, an appreciation for the nonconsumable value of the commons $N(x)$ was found to be important. $N(x)$ was not included in the factorial experiments because $N(x)$ can be enjoyed regardless of whether one Takes or Refrains, and so is irrelevant to an EMAV decision tree. $N(x)$ is really more of an identification with nature than with community, in contrast to $C(x)$, which encompasses any moral considerations. Individual DMs' actions have no effect on their $N(x)$ payoffs, which are determined by the state of the commons, which is in turn affected by the actions of other people. The only way to increase one's $N(x)$ payoff is thus by getting other people to Refrain.

Increasing others' appreciation for nonconsumable values means getting them to add $N(x)$ to their decision trees or increase the weight they give to $N(x)$. This approach was explored by adding $N(x)$ to some of the decision trees. Because it drops out of the decision trees (as discussed in Chapter VI and earlier in this chapter), if $N(x)$ were added to the payoffs for all four DM Types, it would have no effect on the results. It would have the effect of adding a constant to the "fitness" of all DMs, because everyone would get the $N(x)$ payoff regardless of their actions.

However, $N(x)$ can affect the outcome if only some of the DMs include it, because it provides a bonus that gives $\mid \mathrm{DMs}$ who value it more of a chance to be progenitors in the GA. That is, it increases the payoffs for any DM Types that include 
it, and thus their relative fitness scores. Similarly, if all DMs included $N(x)$ but gave it different weights, those who gave it higher weight would get higher scores.

There is a subtle complication with this conclusion that must not be overlooked: $N(x)$ adds a bonus to DM Types that include it, but the only effect it has on the fate of the commons is by giving those Types a boost; only if those Types happen to be Refrainers will it help preserve the commons. If Type IV's include $N(x)$, while Type I's do not, for example, then Type IV's (who always Refrain) will get a bonus for $N(x)$ whereas Type I's (who always Take) will not. Type IV's will then have more of a chance in the progenitor selection lottery. It is important to keep in mind that $N(x)$ does not change anyone's preferences; it just increases the relative attractiveness of being a DM Type that includes $N(x)$. This is particularly so when the commons is in relatively good condition because $N(x)$ increases with $x$.

To simulate the effects of including $N(x)$ in some of the DMs' decision trees, the simulation was modified so that Types III and IV DMs included $N(x)$ in their payoffs. The rationale behind choosing the more "environmentalist" Types was that this was the only way $N(x)$ could be expected to help save the commons; if an $N(x)$ bonus were given instead to all DMs, it would make no difference to the outcome, and if it were given to only the more "extractor" Types I and II, it would tend to accelerate the commons destruction. Throughout this work the "burden of proof" has been left to Hardin: when assumptions have to be made, they are made in favor of any solution that is being simulated because Hardin said nothing will work unless population is 
controlled. If even these optimistic assumptions do not produce solutions that are robust to population growth, it is less likely that they would work for more realistic assumptions.

Table VIII shows the total payoffs assumed, including $N(x)$. A reference run, without $N(x)$, is shown in Figure 8.19. The simulation in Figure 8.19 lasts 117 iterations ${ }^{14}$, and near the end of the simulation, the population consists of pessimistic Type I DMs who prefer to Take, and pessimistic Type III DMs who prefer to Refrain.

\begin{tabular}{|l|l|l|}
\hline DM payoff Type & $\begin{array}{l}\text { Payoff for } \\
\text { Taking }\end{array}$ & $\begin{array}{l}\text { Payoff for } \\
\text { Refraining }\end{array}$ \\
\hline I. Hard core Extractor & $\pi(T)$ & 0 \\
\hline II. DM with mild "Conscience" & $\pi(T)-C(x)$ & 0 \\
\hline $\begin{array}{l}\text { III. Temptable conservationist } \\
\text { with an appreciation for the } \\
\text { nonconsumable commons value }\end{array}$ & $\begin{array}{l}\pi T)-C(x)+ \\
\text { IV. Hard core Conservationist }\end{array}$ & $N(x)+N(x)$ \\
\hline
\end{tabular}

Table VIII. The four DM payoff types and their payoffs. $\pi(T)$ is profit, $C(x)$ is the "Conscience" function, and $N(x)$ is the nonconsumable value funtion.

A histogram of the population just before the crash, at iteration 100 , is shown in Figure 8.20.

\footnotetext{
${ }^{14}$ The scttings for this simulation were the same as for the base case simulation shown in Figure 8.11; the only difference was that the initial population size was 150 , rather than 100 as it was in Figure 8.11. This is why the simulation of Figure 8.19 ended so much sooner than the earlier one.
} 


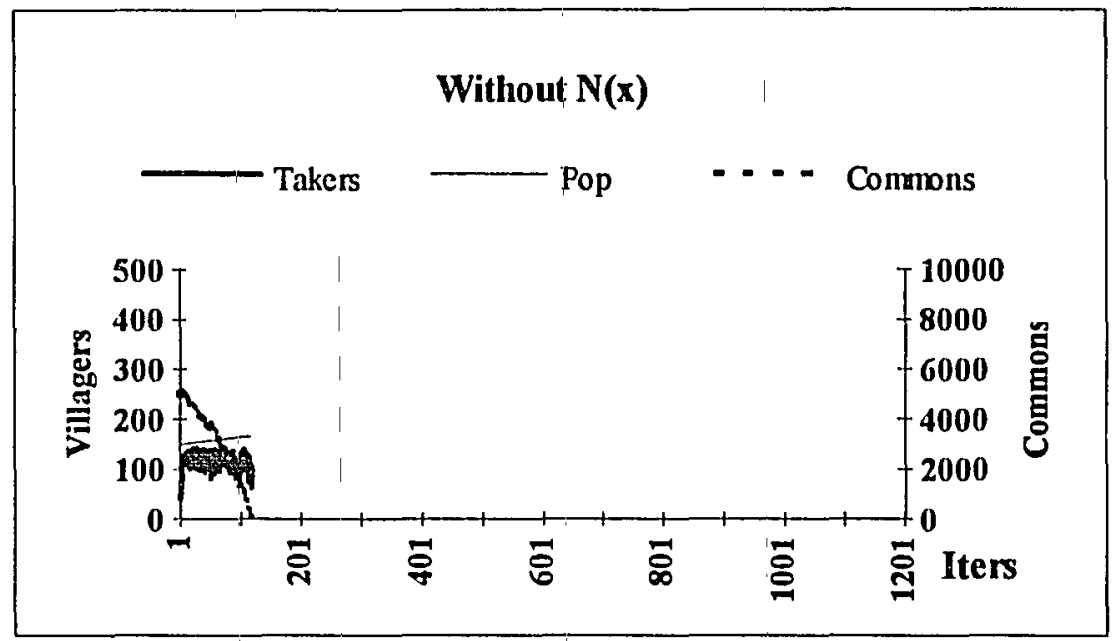

Figure 8.19. Reference simulation, with $m=0.02, k=3.0$, and no $N(x)$. The commons is destroyed by the 117 th iteration.

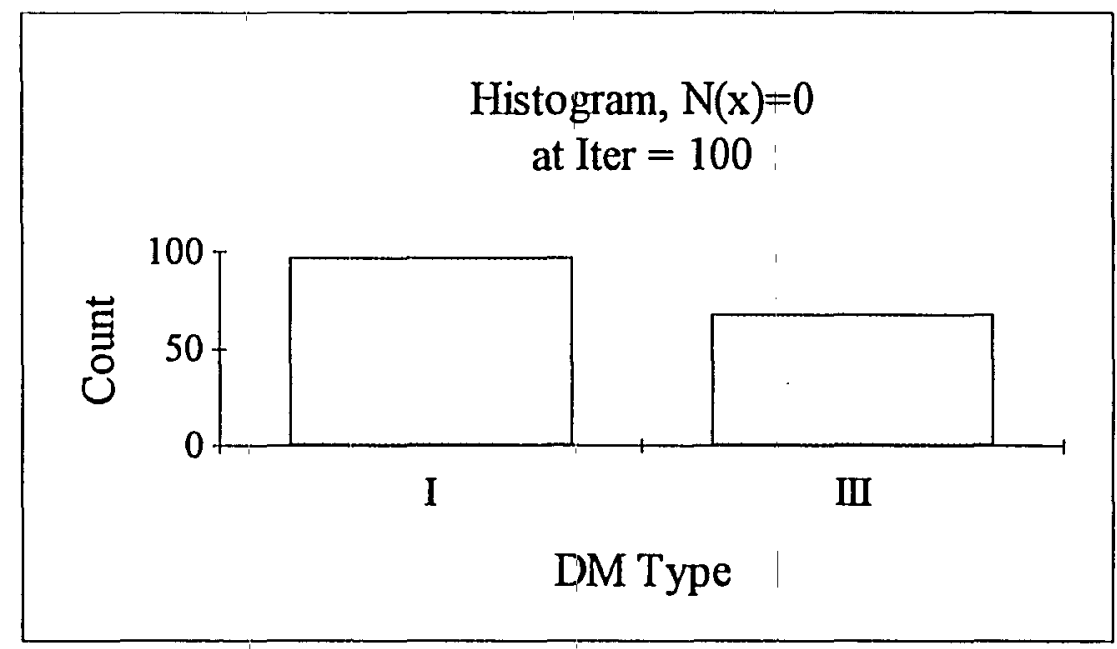

Figure 8.20. Population distribution in reference simulation (Figure 8.19), shortly before the commons goes extinct (Iter 100; $x \mid=1380$ ). All DMs are pessimists, and the 97 IType l's, who receive a higher payoff, prefer to Take, while the 68 Type III's prefer to Refrain. 
In the next simulation, $N(x)$ is added to the payoff functions (see Table VI). The $N(x)$ and $C(x)$ value functions are assumed to be equally weighted, and the slope of $N(x)$ is assumed to be the same as for $C(x)$, with opposite sign. These assumptions are somewhat arbitrary, because it is hard to justify any particular relationship between the two. However, it does not seem unreasonable to assume that people who care about both "Conscience" and the nonconsumable commons product could assign them similar value functions. The same simulation that was shown in Figure 8.19, except with the addition of $N(x)$, is shown in Figure 8.21. The commons lasts 155 iterations longer, but still is destroyed.

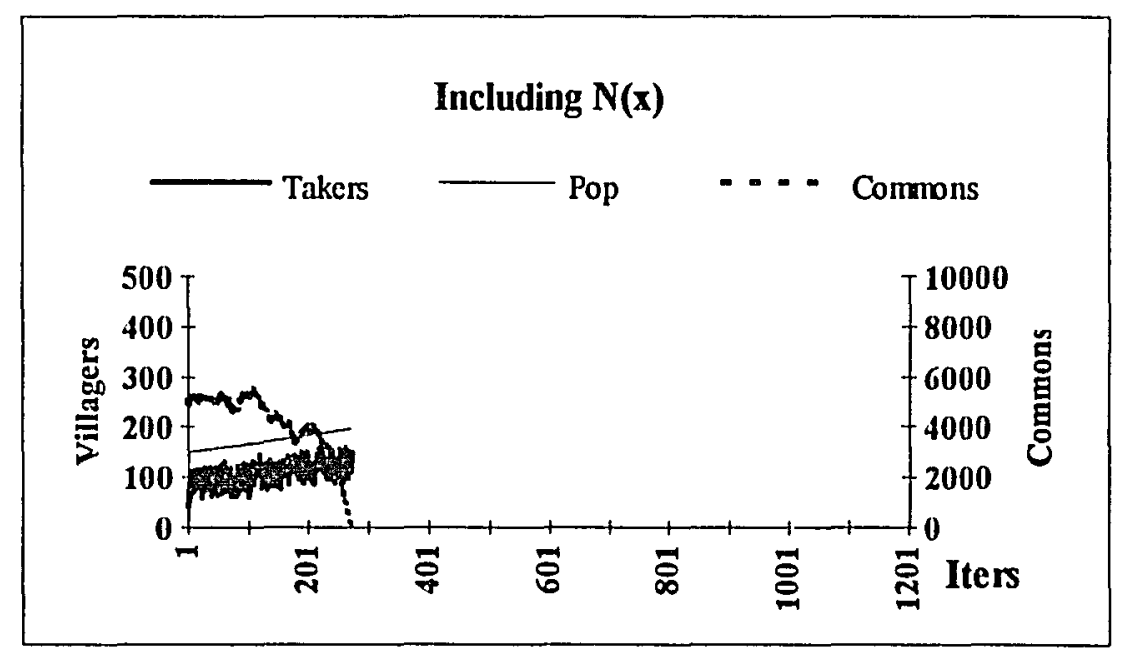

Figure 8.21. Simulation identical to reference simulation (Figure 8.19), but with $N(x)$. 
Two histograms are shown in Figures 8.22 and 8.23: the population at iteration 100, which can be compared to the histogram at iteration 100 in Figure 8.20; and the population shortly before the commons is destroyed, at iteration 272 .

A comparison of the three histograms reveals the impact of $N(x)$ : without it, the population quickly converges to Type I's (who Take) and a smaller number of Type III's (who Refrain). With $N(x)$, the Type IV Refrainers are predominant for awhile (Figure 8.22), at least until demand grows to the point that the Type I's take over (Figure 8.23). The presence of the Type IV's serves|to slow the extraction rate at the time when the commons would otherwise have been destroyed (Figure 8.20). As the population grows and demand increases, not only does potential profit increase, but the payoff from $N(x)$ decreases as the commons declines. Type I DMs regain their advantage, and at the end of the simulation (Figure 8.23), $N(x)$ makes little difference. Doubling the magnitude of $N(x)$ allows the commons to survive even longer (326 iterations instead of 272), but tripling it only allows the commons to survive ten more iterations. Doubling both the $N(x)$ and $C(x)$ parameters allows the commons to suvive 611 itrerations, a significant improvement.

$N(x)$ and $C(x)$ could increase like this together, if, for example, society perceived increasingly important moral as well as aesthetic reasons for Refraining, but either could increase alone. Aside from the simulations in this section to examine the role of $N(x)$, all the other simulations ignore $N(x)$ 
Histogram, with $\mathrm{N}(\mathrm{x})$

at Iter $=100$

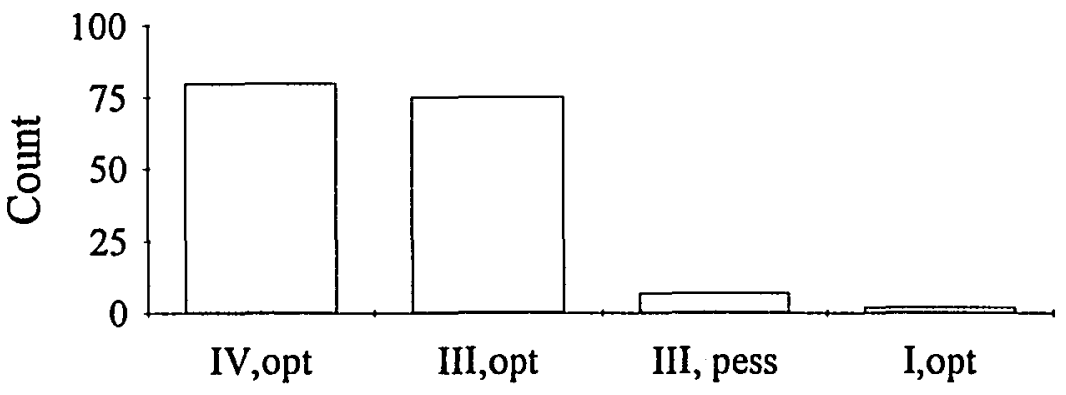

DM Type

Figure 8.22. Population distribution at iter $=100$, with $N(x)$. The hard core, optimistic Refrainers, Type IV's, are the most numerous, and most of the rest of the population are Type III's. The commons is in moderate condition ( $x=$ 5104).

\section{Histogram, with $\mathrm{N}(\mathrm{x})$ \\ at Iter $=260$}

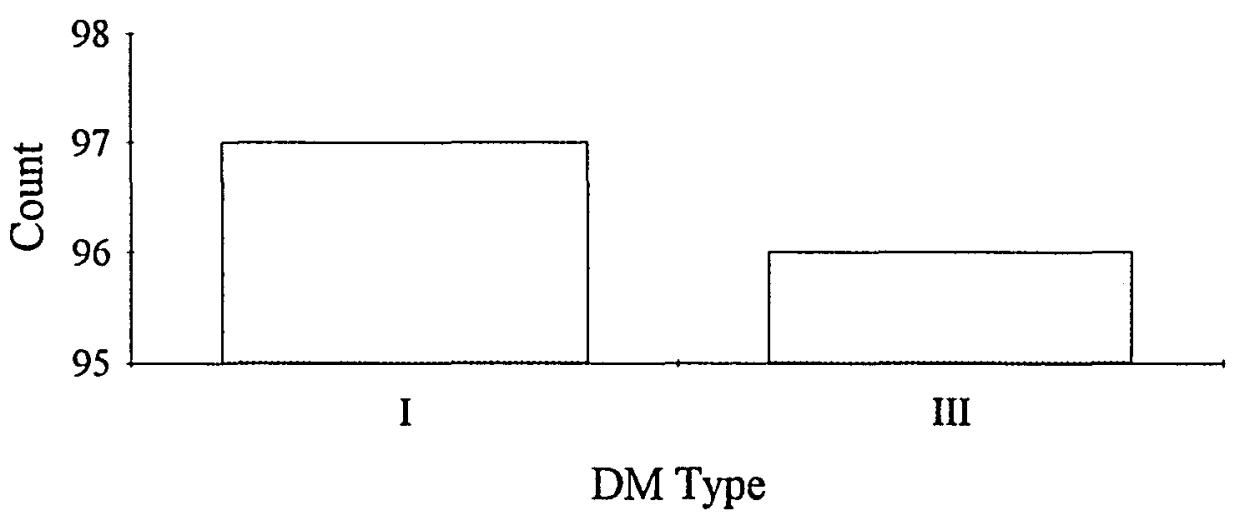

Figure 8.23. Population distribution just before the commons is destroyed ( $x$ $=260$ ). Note the similarity to the population distribution without $N(x)$ shown in Figure 8.20: with or without $N(x)$, at the end, all are pessimists, and the Type I Takers outnumber the Type III Refrainers. 
These simulations consistently suggest that "Conscience" alone could make a significant difference in how long the commons survives.

$N(x)$ alone cannot. Including only $N(x)$ and not $C(x)$ in the simulations produces the same results as including only profit; without $C(x)$, Taking is always dominant. It is $C(x)$, not $N(x)$, that affects a person's choice. In this crude analysis, $N(x)$ was only added to the payoffs for the two most conservationist DM Types (III and IV) ${ }^{15}$. There is then a greater proportion of Types III and IV when the commons is in good condition, because $N(x)$ gives them a competitive advantage over the Types that do not get the $N(x)$ bonus. If $N(x)$ had instead been given to Types I and II, it would have accelerated the destruction of the commons because Types I and II tend to always Take; if it had been given to all four Types, it would have made no difference at all. The purpose here was not to do a rigorous analysis of nonconsumable values, but to roughly investigate how $N(x)$ could help save the commons.

The game theory analysis showed that payoff structures that include $N(x)$ can cause the payoff structures of the Type I and II players to be like the PD; but with both, or with only $C(x)$, Chicken-like games more tractable to solution are possible. Here, $N(x)$ was only added to Types III and IV, which does not significantly change the game form of their payoff graphs. What all this suggests is that people hoping to save a commons might be more successful if they concentrate their efforts more on matters of

\footnotetext{
${ }^{15}$ A more rigorous way to include $N(x)$ would be by adding a gene to the genotypes so that $N(x)$ could vary independently, just as does $C(x)$. This would have required significant time and effort to revise the computer code, however, and the problem of functional form is substantial.
} 
community or moral concerns, than aesthetics, particularly when the commons condition has already become degraded.

This is not the approach that has always been used. The big environmental organizations such as the Sierra Club and Audubon Society were started in order to secure hunting and fishing reserves on public lands for mostly wealthy, white men (Dowie, 1995). It is hard to imagine an environmental group advocating setting aside public lands for the use of hunters today. The emphasis in the past 30 or so years has been more on hiking, fly-fishing, and other low-impact activities - reflecting $N(x)$ kkinds of values, although many in the environmental movement also express values that sometimes sound almost religious. Concern for the rights of future generations of people as well as other species is being expressed more often, too, but members of environmental groups are perceived in many rural areas (and in the poor countries of the world) as self-righteous, urban, elitist, backpackers, and not as soft-hearted dogooders (Dowie, 1995). If these groups were to increase their level of preaching about the immorality of rural lifestyles dependent on resource extraction, they would probably be very poorly received ${ }^{16}$.

Matters of conscience can be tricky to promote: Refraining because one believes it is the ethically right thing to do is not the same as lecturing others that they should Refrain because you believe it is the ethically right thing for them to do. In the

\footnotetext{
${ }^{16}$ When Andy Kerr, an outspoken environmental leader in Oregon, moved to a timber town in 1994 and began preaching to the laid-off timber workers about the destructiveness of their lifestyles, he received numerous death threats and his $\log (!)$ cabin was polted with eggs.
} 
simulations, decision models that provide more total satisfaction become more prevalent simply because they provide higher payoffs; the mechanism is assumed to be a social transmission mechanism analogous to biological recombination. DMs become more "Conscience"-driven essentially because being more "Conscience"-driven becomes more appealing. When "Conscience" and an appreciation for nonconsumable aspects of the commons add to total payoffs for Refraining, then the decision trees that provide those higher payoffs become more popular. Until, that is, population grows enough that demand offsets their beneficial effects.

\section{Financial incentives}

Financial incentives - penalties, fines, tax incentives and bonuses - are elements of the solution approaches that rely on changing the actual monetary payoffs in the TOC. The motivation behind this approach is to try to internalize the externalities: to get decision-makers to take into account the true costs of their actions.

In this approach, a bonus of some kind is paid for Refraining, or a penalty is assessed for Taking. The idea is that people will then Refrain because they want to. Some kind of coercion (i.e., government, or at least within-group self-enforcement) is required, which implies that people may actually feel that they have to, rather than that they want to. If the level of coercion required for enforcement is too high, then not enough people will comply for the solution to work; for some, the expected cost of noncompliance is less than the expected cost of compliance. 
To simulate the reward/penalty approach, a penalty function for Taking, and/or a bonus function for Refraining, could be added to the computer model that increases as $x$ declines. This is, incidentally, exactly what $C(x)$ does. $C(x)$ can even be viewed simply as a penalty/reward function in the utilitarian, rather than the moral, sense.

A thought experiment can predict the outcome of this approach: just as with $C(x)$, some reward/penalty function could certainly be identified for any population size that would cause a sustainable harvest level to be achieved. Then, as population grows, the reward/penalty function would have to grow, too, in order to offset the increase in demand. Either it would have to be linked to population as is profit, so that it would grow automatically with population, or the function parameter(s) would have to vary as $k$ or $m$ did in the solution simulations shown above. Graphs of such simulations would then exhibit the stepwise ratcheting effect that solutions based on decreasing $k$ or increasing $m$ produced.

Just as with the above examples, however, as demand grows, and larger penalties or rewards are required, voluntary Refraining would become increasingly problematic. Objections would increase, not only from would-be Takers who resent the big penalties, but also from the rest of the population whose taxes must be used to pay the rewards. Enforcement would become increasingly difficult.

\section{Property rights}

If common access is the problem, then property rights advocates argue that the 
solution must be to eliminate public property. Privatizing the commons could be implemented by selling it off, or somehow limiting public access in such a way as to allow access to be bought and sold as is private property. Land-based or enclosable commonses such as forests or rangeland would be straightforward to privatize, in theory, because it would be methodologically clear who owned what: for example, fences could be built. The "downstream" costs of grazing and logging (such as water pollution and aquatic habitat destruction) would not be accounted for, and it could be argued that this upstream-owner versus downstream-victim dichotomy can be viewed as a kind of commons dilemma itself. However, to property rights advocates, if upstream activities damage the property values of downstream property owners, then the legal system can be used to get the polluters to compensate the "pollutees." At least, they argue, property ownership and access are clear, because someone can be identified who is directly affected, as well as who is directly responsible.

Other commonses, such as fish, groundwater, and clean air, are more difficult to privatize because delimiting ownership is virtually impossible. Artificial property rights are sometimes created that can be sold or traded, the idea being that if the linkage between supply and demand, or beneficiary and victim, is established, even artificially, then the market can solve the commons dilemma. For example, ocean fishery managers in New Zealand and Alaska have tried issuing tradable fishing access rights called Individual Transferable Quotas (ITQs) with little success, so far, mainly because of the enormous political pressures and enforcement problems involved (Duncan, 1995; 
Mathews, 1995; Mace, 1993; see Chapter IV).

Simulating private property solutions would require significant modification of the computer model, and since privatizing the commons implies "solving" the TOC by eliminating it, this "solution" was not simulated. However, this area of potential solutions would be a potentially fruitful line for further work, particularly because it seems reasonable to expect that population growth would have effects on any private property solutions similar to those it has on common property solutions.

The main difference between the commons decision modeled by the influence diagram in Figure 3.2, and the decision of a private property owner, is that in the TOC model, the state of the commons is determined by the actions of others, not the DM; but in a private ownership model, the state of the (owned) resource is determined primarily by the actions of the DM because other potential Takers are excluded from access. An interesting simulation that comes to mind would be to include in the model the "downstream" stakeholder who has no access to the resource, but whose welfare is determined by the "upstream" resource owner. Some questions to investigate would be what role a "Conscience" function could have in regulating the actions of the "upstream" DM, and how population growth might affect that and other possible solutions such as financial incentives.

\section{$\underline{\text { Regulatory control }}$}

Some kind of governmental system is necessary for any of the solutions to be 
implemented, with the possible exception of Hardin's historical state, where the population was so small relative to the regeneration capacity of the commons that it did not matter what anyone did. "Some kind of government" could be a council of village elders, a shaman, a feudal lord, or a Federal Department of the Interior, and its power could range from merely advisory to strongly coercive. However broad the range of the "government" solution might be, in this work, the "Regulatory control" category is assumed to mean relatively coercive solutions where a clearly identified authority determines an acceptable level of harvest, and enforces that level by means of licenses, regulations, fines or jail terms, similarly to the way state Departments of Fish and Wildlife license and regulate sport and lcommercial fishers (see Chapter IV).

It is worth noting that solutions that create a kind of artificial property rights, such as the Individual Transferable Quota (ITQs) in ocean fisheries, also rely on penalties and/or rewards for enforcement. As long as the resource harvest level is determined and administered by some regulatory body, a DM has to trade off whether it is preferable to follow the rules of the property rights system, or to not follow the rules and run the risk of getting arrested or otherwise paying some penalty. The expected payoff for breaking the rules depends on what the actual penalty would be, as well as on the probability of getting caught.

To investigate such a regulatory solution, a simulation was developed that enforces a harvest limit by assessing a penalty on lawbreakers who get caught. A maximum allowable total harvest level MaxTakers is set, and in each iteration, a penalty 
is imposed on any DM who chooses to Take after the catch limit MaxTakers has been reached. The penalty is set equal to the negative of what the profit would have been. The illegal Taker who gets caught receives a payoff of less than zero, and the illegally harvested product is confiscated. This is what happens in the real world when a DM gets caught with illegally caught fish: the fish are already dead, but the fisher, who does not get to keep them, has to pay the penalty.

This way of simulating harvest limits is admittedly simplistic. Pollution limits are like harvest limits if the polluter is thought of as "harvesting" clean air; grazing commonses are regulated via grazing permits issued to individual ranchers; timber commonses are regulated by auctioning off parcels to private logging companies, and so on. There are many different ways of imposing harvest limits. In fisheries, gear restrictions or per-boat or per-fisher limits are perhaps the most common. Restrictions are enforced via fines, gear confiscation, and occasionally jail terms. However, a very common approach, particularly in Alaska and Pacific Northwest ocean salmon fisheries, is to open the fishery to a short-term free-for-all until a set number of fish are caught, and then to shut it down. That is what is simulated here.

In deciding whether or not to fish, DMs have to estimate the probability and likely payoff of being legally successful, as well as the probability and likely payoff of getting caught if they go on to fish illegally. The general situation facing anyone making a decision about exploiting a regulated commons is similar enough that the general conclusions of this simple simulation should apply to other commonses as well. The 
decision tree on which the simulation is based is shown in Figure 8.24 .

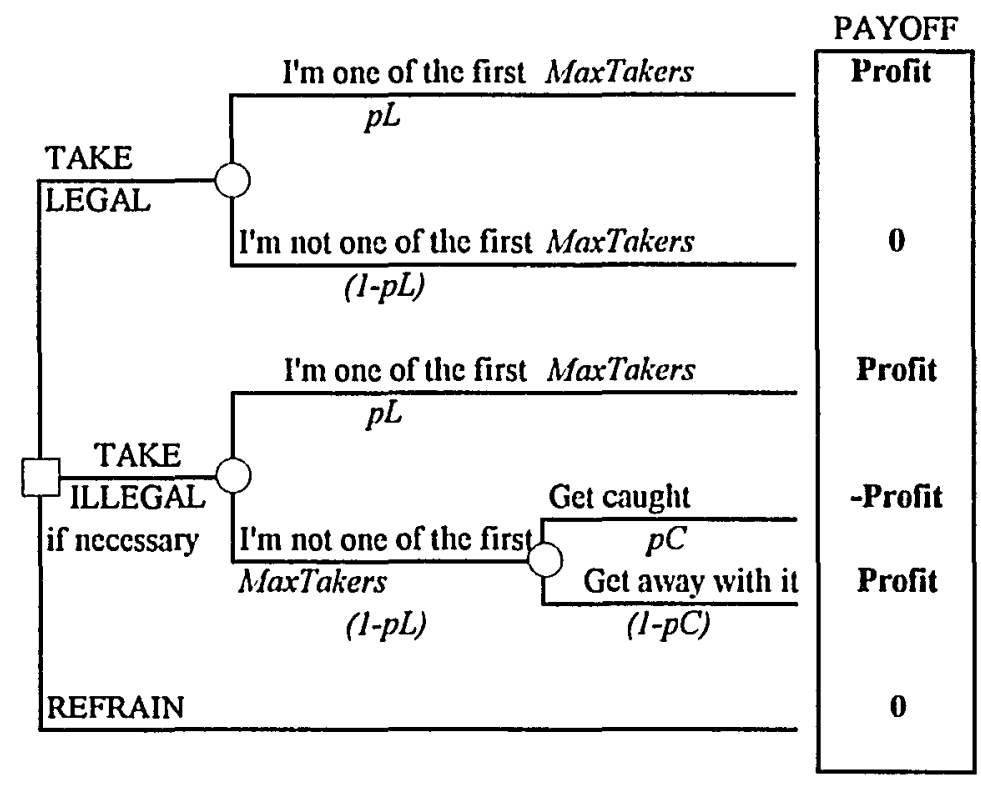

Figure 8.24. Decision tree for fishers in a "free for all" fishery subjected to catch limits and potential penalties. Only the profil attribute is included. $p L$ is the probability of catching a unit of fish during the legal period, and $p C$ is the probability of getting caught, given that a DM was not among the legal Takers and fished anyway.

In the decision tree of Figure 8.24, it is assumed, as it was in all the previous simulations, that no Taker continues fishing after catching their fish. DMs who fish illegally receive the profit as usual if they do not get caught. If they do get caught, then they loose the profit and instead pay a penalty that is equal to the opposite of what the profit would have been. In this way, the penalty, like the profit, increases as the population grows.

The $E V$ of Refraining is zero. This village is assumed to be made up entirely of 
Type I DMs (i.e., "Conscience" and $N(x)$ are neglected, primarily to keep things simple). In these simulations, some are optimists who believe either that they will succeed during the open season ( $p_{L}$ is high), or that they will not get caught if they go on to fish illegally ( $p_{C}$ is low): they will Take, legally or illegally. Pessimists believe they either will not succeed during the open season $\left(p_{L}\right.$ is low) or will get caught if they fish illegally ( $p_{C}$ is high); they tend to Refrain. The GA thus selects for pessimism or optimism, rather than for the relative weights given to the profit and "Conscience" attributes.

Leaving out "Conscience" leaves out some potentially interesting effects in this solution category, but including "Conscience" would require fairly extensive implementation effort. $C(x)$ could certainly be affected by the legality of one's choice, as well as by the perceived fairness of the imposed restriction. Harvest regulation and people's responses to it is an extensive field of research that is outside the scope of this dissertation, but this simulation could be very useful for simulating different regulatory schemes and possible human responses. The present simple effort is intended to be only a preliminary investigation into how the simulated DMs might respond to penalties.

In the simulation code, the sequence in which the DMs make their decisions is random, and so the probability of being one of the DMs who gets caught is equal for all. Recall that the sustainable harvest level is 100 units per iteration. If the population consists of 150 potential Takers, and they all decide to Take, then the true probability $p_{L}$ of being among the first 100 Takers is $100 / 150$. Calculating $p_{L}$ in advance is not 
quite that straightforward, though, because in any iteration it is difficult to say in advance how many will decide to Take. The actual probability of being among the legal MaxTakers is unknown because it depends on how many others end up Takeing. Assumed values for $p_{L}$ and $p_{C}$ were varied in the decision trees, and the results are described below.

Although a rigorous experiment was not conducted, two results were clear enough, and reasonable enough, to support relatively strong conclusions: in order for the harvest limit approach to prevent the commons destruction, (1) the perceived probability of getting caught has to be high, and (2) unless the perceived probability of getting caught is $100 \%$, MaxTakers has to be held lower than the desired harvest level. The less effective the perceived enforcement, the lower MaxTakers has to be. Three typical runs are shown in Figure 8.25.

Although not shown, histograms of the distributions of DM Types during simulation runs indicate that only when the population is relatively pessimistic do they avoid destroying the commons. This solution is stable with a growing population because the penalty function, like the profit function, is proportional to demand. If the penalty is instead set proportional to the level of the commons, it will have the same effect as the guilt impact of $C(x), w$ ith the important exception that it would apply to everyone, including Type I's. Unfortunately, such a solution will not be stable with increasing population. That is because demand grows with population, and if the 


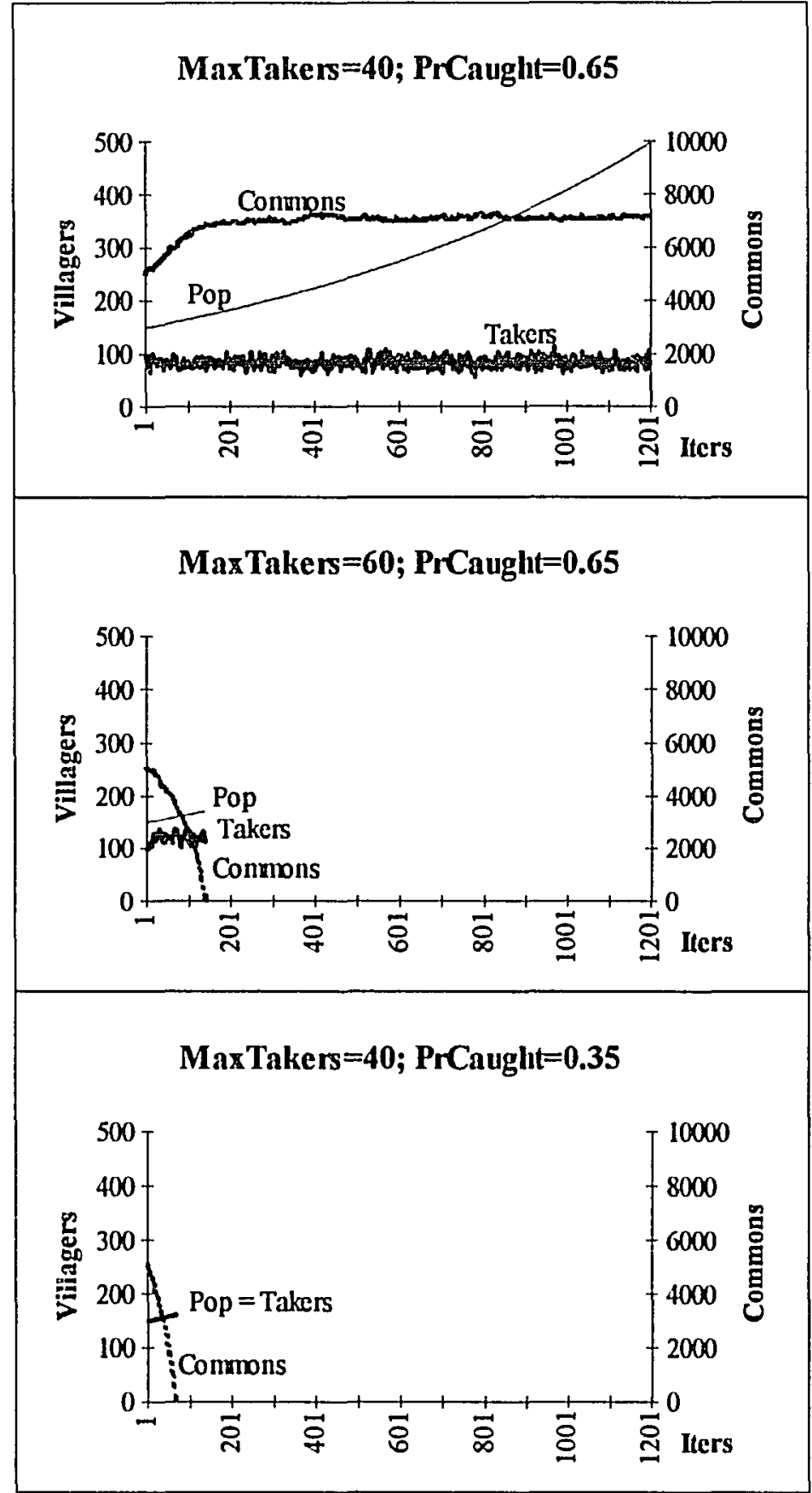

Figure 8.25. Typical results of simulations where the harvest levels are set at MaxTakers, and the perceived probability of getting caught for harvesting illegally is PrCaught. The commons tends to survive longer when MaxTakers is low, and/or PrCaught is high. 
penalty does not, then demand will always eventually cause the expected profit to exceed the expected penalty, even with perfect enforcement.

These findings are hardly surprising. The more badly someone wants or needs something, the more likely it seems to be that they might break the law: if the potential costs of lawbreaking are less than the potential benefits of selling a highly profitable product, even a decision analyst might recommend crime. Only a penalty function that is tied to demand, rather than to the state of the extractable product, appears workable over the long run, but such a penalty function seems unrealistic because it is unlike any kind of familiar regulatory approach. Taxes on resources bought or sold may increase as profits increase, but in order to offset the increase in demand due to increasing population, the tax rate (i.e., percentage of net profit) would have to increase with demand. Regulatory penalty functions more familiarly depend on the state of the commons, as does $C(x)$, or on the magnitude of the crime (illegally harvesting one fish does not carry as large a penalty as illegally harvesting 100 fish).

An interesting implication of the need to tie the penalty function to demand rather than the state of the commons is that if a "Conscience" function could somehow be tied to population size, it could also work for a growing population! "Conscience" is, after all, a penalty function for Taking and a reward function for Refraining, but in this model it is tied to the state of the commons and not to the number of potential Takers. Recall that the reason "Conscience" is a function only of the state of the commons is that Hardin $(1968,1971)$ claims that environmental ethics depend on the 
state of the commons rather than the number of people. However, if enough people feel more virtuous for Refraining when the village begins suffer the effects of overpopulation, and/or feel guilty for Taking, then it is possible for a "Conscience"based solution to be stable over a longer period.

People do sometimes behave more altruistically when struck by disasters, particularly in close-knit communities — at least, up to a point. Other people engage in looting and theft when the opportunity is created by a hurricane or earthquake. Starving homeless in the streets of Portland, Oregon, are fed by some passers-by and spit on by others. Some people believe more food and medical aid should be sent to countries in Africa with ballooning populations of mostly starving people, others disgree, asserting that the misery in such countries is the poor people's own fault. If the number of starving homeless continues to increase, will people's compassion, let alone their willingness to do without in order to help those in need, increase? In the current political climate, the answer does not appear to be encouraging.

\section{Conclusions}

The model on which these solution simulations are based is very simple. There are only a few variables that are important in the baseline simulation: the population growth rate, the commons regeneration rate and stochasticity, the slope and magnitude of the demand curve, the slope and magnitude of the "Conscience" function, and the population diversity control parameters. The problem being analyzed is very complex, 
and the solutions proposed are complex. There are only a few variables in the computer code that could be used to simulate a variety of complex phenomena, and yet the results are intuitively reasonable, if not exactly reassuring.

The goal of these solution simulations was not to determine definitively whether any particular solution would work in the real world, but rather to investigate the question, "Was Hardin right?" — in other words, does it seem reasonable to expect that the problem of natural resource depletion can be solved over the long run unless population growth is stopped? Hardin says the answer is no. It seems difficult to believe that the correctness of Hardin's answer to the question is not obvious; yet the debate rages on in professional journals, and even within professional newsgroups on the Internet. Even people struggling to feed themselves continue having children, corporations continue trying to encourage consumption, and efforts to control people's reproductive freedom are viewed as violations of fundamental human rights. If Hardin is right, people do not behave as though they believed him, or as though they thought their actions mattered.

Many people apparently believe that technology will allow people in the rich countries to continue to live and act as we have always done, as well as to allow poor countries to improve their standards of living. In the simulations, the contribution of technology was simulated by increasing supply via the regeneration rate constant $r_{0}$, and increasing the availability of substitutes and/or efficiency of use via decreasing the demand function parameter $k$. The simple simulations of technological solutions can be 
made successful for any population size; but if population continues growing, then technological solutions have to grow as well. If population growth is exponential, then so must be the decrease in demand, increase in efficiency of use, increase in commons regeneration rate, or production of new substitutes. The evidence so far is not encouraging. Technological fixes have helped over the short run in some areas, but there are no examples of any commonses that have been rescued by technology as yet.

Community identification is a difficult idea to simulate, and the simple approach used here was to use the "Conscience" function to represent the importance of community, or other moral concerns. In the game theory analysis, the "Conscience" function was instrumental in allowing games like Benevolent Chicken and Hero to develop. The payoff orders in these games actually favor payoffs to others under some situations. Much like the solutions based on increasing the contribution of technology, increasing the impact of "Conscience" can also be shown to work for any population size. Yet in order for "Conscience" to work as population grows, each new generation has to be more "Conscience"-driven than previous generations, because in order to counteract the impact of growing demand, "Conscience" has to grow at the same rate. Unfortunately, if the community exhibits an increased commitment to preserving an increasingly scarce resource, a kind of arms race develops because of the increasing demand to reduce restrictions and allow more harvesting. A simple game theory analysis indicates that the tension between extractors and preservationists is likely to drive a cyclical pattern such as has occurred over the past 30-50 years in the United 
States. Unfortunately, while there is plenty of evidence that demand increases with growing populations, there is little evidence to support any contention that the importance of "Conscience" ever increases with growing populations, even in the face of certain disaster.

Adding the nonconsumable attribute function $N(x)$ to some DMs' decision trees helps increase the proportion of DMs who prefer to Refrain, and thus to preserve the commons longer than it would otherwise have lasted. As a first approximation, it was assumed that only the more environmentalist-type DMs, Types III and IV, include $N(x)$ in their decision trees. If $N(x)$ were included for all four Types, $N(x)$ would make no difference in the outcome, because the payoff due to $N(x)$ is the same regardless of whether a DM Takes or Refrains. $N(x)$ helps save the commons only because it gives a payoff bonus to Types III and IV DMs, and helps them stay in the population pool, at least for awhile. Unfortunately, $N(x)$, like $C(x)$, does not increase with population. As demand grows, the relative importance of $N(x)$ declines. The only way $N(x)$ could actually help save a commons in the real world is if the demand for the nonconsumable aspects of the commons were to increase more than the increasing demand for the consumable product of the commons. The opposite assumption appears to be more supported by the real world.

A great variety of approaches that rely on regulatory control was crudely simulated with one very simple model of a "free-for-all" fishery subject to a harvest limit, with penalties imposed on violators who get caught. The intriguing thing about 
this solution is that it is the only solution simulated that relies on a function of the number of Takers, rather than on the state of the commons. Because the penalty function is simply the negative of the profit function, it grows with population and thus is stable with population growth. Not surprisingly, the outcome depends on enforcement effectiveness and the actual level of harvest limits imposed. If such a penalty function could be imposed, it seems reasonable to believe that it might work: but it seems very unlikely that any political system could long sustain it. As demand for a scarce product increases, pressure must also increase on its regulators to allow more of it to be harvested. Resource managers in the United States have had little success resisting such pressures, as recent conflicts over public grazing, logging, air and water pollution controls, and ocean fisheries attest. 


\section{Chapter IX}

\section{SUMMARY AND CONCLUSIONS}

Garrett Hardin said the reason humans destroy commonly-held, important resources is because of a simple cost-benefit analysis all of us who are "rational" must do (Hardin, 1968): if I add a cow to the commons (or cut a tree in the public forest, or catch a fish, or drive a car), I get all the benefit, but the costs are shared among many. My benefits are +1 , and my costs are nearly 0 . If I do not add a cow, I get nothing. Everyone else benefits from my self-restraint, and I may feel like a fool. Since everyone reasons this way, he said, the ultimate destruction of the commons is inescapable. Hardin argued that because of the inescapability of this logic, social, economic, or technical solutions are doomed to fail unless human population growth is controlled. This is the Tragedy of the Commons.

In order to investigate the question, "Is Hardin right?" this dissertation integrates well-established tools from disparate fields to model the evolutionary interactions between the decision-makers and the commons: expected multiple-attribute value (EMAV) theory from the field of decision theory to model human decisionmaking; modeling methodology from the field of ecology to model a stochastically 
varying, simple natural system; and the genetic algorithm from the fields of artificial life and optimization. This work contributes to theory and practice in all these fields not by inventing a completely new computer methodology, but by innovatively integrating methodologies already well established in their respective fields.

Considering Hardin's lifelong battle with neoclassical economists, it is interesting that his seminal paper was based on a neoclassical economic model of human decision-making. To advance his model a step further, this dissertation assumes that decision-makers may consider attributes other than pure profit, and that they explicitly take uncertainties into account by using expected multiple attribute value (EMAV) decision trees to make their decisions. A decision-maker (DM) who chooses to harvest a unit of the commons is called a Taker, and one who decides not to is called a Refrainer. "Harvest" means any consumptive activity such as catching fish, cutting down trees, polluting air or water, or grazing cattle.

Three attributes are considered: profit, nonconsumable value, and "Conscience". Profit is not treated as a constant +1 as Hardin assumed. Instead, marginal profit varies inversely with supply. Hardin neglected costs such as depreciation and labor, arguing that extraction costs are effectively zero because they are diffused among so many other herdsmen. In this work, extraction costs are subsumed in the declining marginal returns of the profit function.

Ecological economists sometimes add a monotonically increasing nonconsumable value attribute $N(x)$ (Pearce and Moran, 1994). $N(x)$ represents 
nonconsumptive uses such as hiking through an old-growth forest or across a grazing commons, enjoying the knowledge that a wilderness exists even if one might never see it, or saving a commons for later. $N(x)$ is an increasing function of the state of the commons $x$, and its value is unaffected by whether a particular DM chooses to Take or Refrain - only by what a great many DMs choose in aggregate. To a particular DM, $N(x)$ is thus not what an economist would call a marginal variable, as is profit (Boyle and Bishop, 1987). As a nonmarginal attribute, $N(x)$ drops out of the decision trees, and thus is not included in the base simulations of this work. It does play an important role in the game theory analysis: only if it is included can the Prisoner's Dilemma (PD) and Chicken develop.

A third attribute, "Conscience", is not discussed as an attribute per se in economics literature, but community and moral concerns are often presented in other literatures as an important mechanism for solving commons dilemmas (Collins and Barkdull, 1995; Daly, 1980; Etzioni, 1988; Sagoff, 1995; Stern, 1985-86; White, 1995). "Conscience" is included as an attribute here, where it represents the guilt some DMs might feel for Taking or the sense of virtue they might enjoy for Refraining. It may arise from concern for the rights of other people or other organisms (now and in the future), or from deontological moral or religious beliefs (Daly, 1995; Ehrenfeld, 1981). Like profit, "Conscience" is also subject to declining marginal returns, but unlike profit, which is a function of the number of Takers, "Conscience" is a function of the state of the commons $x$. The marginal "Conscience" function $C(x)$ is assumed to be 
a monotonically decreasing function of $x$, in contrast to $N(x)$, which is an increasing function of $x$.

The way the DMs incorporate uncertainties and the weighted attribute functions into their EMAV decision trees is determined by a structure in the computer code called a "genotype." The DMs range from a hard-core extractor Type I, who only considers profit (a Hardin herdsman), to a hard-core preservationist who only considers "Conscience" and the nonconsumable value (Type IV). Each of the four Types has an optimistic version and a pessimistic version. The genetic algorithm (GA) simulation method is used to simulate social evolution, whereby the distribution of beliefs and values in the population varies over time. The variation is driven by a selection mechanism called the "fitness function." At the end of each iteration, this "fitness function" uses the relative payoffs achieved by the DM genotypes to modify the distribution of genotypes for the next iteration, so that genotypes that lead to higher net payoffs become more prevalent. Payoffs are determined by the actions of other DMs, the state of the commons, and the DMs' single attribute functions and attribute weights. The GA provides a feedback loop that links the DMs' beliefs and values to the state of the commons.

The commons is simulated as a simple, stochastically regenerating public-access resource. In each iteration of the simulation, each DM who decides to Take depletes the commons by one unit of $x$. This depletion is countered via the commons regeneration rate. If the total harvest is more than the total regeneration, then the 
commons declines during that iteration; then the $C(x)$ payoff increases and the $N(x)$ payoff declines. The profit the Takers get is determined only by the total number of Takers; but the "Conscience" and nonconsumable value payoffs are determined by the state of the commons.

For harvesting of any renewable resource to be sustainable, the harvest rate must mostly stay below the resource's ability to regenerate, including enough margin for inevitable natural stochasticity. Hardin said that the only way this can be achieved for any commons over the long run is if population is maintained below the commons carrying capacity. Technology may increase the carrying capacity, either by improving the commons regeneration rate, or by creating more efficient ways to use the product and/or manage the waste, but his fundamental thesis was that population belongs to the class of problems for which there is no technological solution.

Six overlapping categories of solutions to the TOC were investigated in this work: technology, community identification and moral concerns, property rights, coercion, changing the payoffs, and population control. A seventh, nonconsumable value, was identified as potentially significant by the game theory analysis.

The three objectives of this work were:

1. To develop an evolutionary computer model that can be used to shed new light on the Tragedy of the Commons.

2. To investigate what variables have the most impact on the outcome, and how they interact with one another. 
3. To investigate the conditions under which the Tragedy of the Commons can be avoided.

\section{SHEDDING NEW LIGHT ON THE TRAGEDY OF THE COMMONS}

Three particularly interesting insights were gained during this investigation: the importance of diversity; the requirement that solutions be driven by population growth, rather than the state of the commons; and the fact that the game theory analysis and the decision theory simulations revealed different, important aspects of the TOC.

\section{Diversity}

The most intriguing insight that resulted from this work is that Ashby's Law of Requisite Variety (LRV) applies to the TOC: diversity in the population is a critical factor in determining whether the DMs even have a chance to deal effectively with a changing commons. The factorial experiments (Chapter VII) as well as the game theory analysis (Chapter VIII) indicated that the parameters in the simulation that control diversity are among the most important.

The reason diversity is important is that unless diversity is maintained in the population, the simulated village is unable to deal with a varying commons. Just as in the real world, the commons in this simulation varies because of human impacts as well as because of natural stochasticity. The more stochastic the commons component of the 
model, the more important diversity is. Diversity is maintained in the real world when a society protects and even encourages contrary viewpoints, and when it invests in efforts such as funding basic research and higher education, that work to constantly (re)introduce diversity into the population.

The garne theory analysis showed that diversity is necessary for a different reason: the attainment of a pareto-optimal outcome. If the entire population has a dominant strategy, as they would in the PD, all will Take or all will Refrain. All Refraining is a trivial solution, because it is assumed here that some level of Taking is desirable: otherwise the slope of the marginal profit function would be relatively flat, which would not represent the importance of commonses relevant to this work such as air, water, forests, fisheries, grazing land, and topsoil. All Taking is no solution either, for two reasons: (1) destroying a commons such as breathable air or potable water probably means human extinction; and (2) even if an economic case can be made for liquidating the commons (Fife, 1971), this work assumes that a solution implies preventing commons destruction.

If everyone has payoffs consistent with Chicken, Benevolent Chicken, or Hero, then there is no dominant strategy: everyone prefers to do the opposite of whatever everyone else does. For example, if everyone were an optimist following an expected value rule, all would Take. If everyone followed the maximin principle from game theory, all would Refrain. Even a population made up entirely of people who follow Kant's imperative or the golden rule would not sustainably utilize the commons. 
A particularly intriguing finding related to diversity is that when "Conscience" is a factor in the decision trees, the dominance of the Taking option in the equivalent payoff graphs changes for some DMs as the commons becomes degraded. When the commons is in good condition, their payoff graphs for Taking are always above their graphs for Refraining (even though their payoffs for Taking if all Take are less than their payoffs for Refraining if all Refrain - the deficient outcome typical of the PD). However, when the commons becomes degraded, these DMs' preferences change so that their graphical payoff structures are what Hamburger (1973) calls "Chicken-like": the payoff graph for Taking crosses the payoff graph for Refraining, indicating that there is no dominant strategy.

The implication is that as long as the worst case outcome is seen as not too disastrous, some people may still be willing to accept the non-pareto-optimal outcome typical of the PD; but if the worst case outcome is seen as utter disaster, then more people may be willing to Refrain, as is typical of Chicken. Without "Conscience", the payoff graphs never cross. In order for the tragedy to be avoided, the population has to have enough people with a "Conscience", or with some other attribute function that increases as the commons declines, that they will stop harvesting once they recognize the consequences of their actions. The importance of diversity would be missed by models of the TOC based on N-person PDs, or any other homogeneous payoff structure. 


\section{Solutions as a function of population growth}

Diversity is a necessary, but not a sufficient, requirement for a solution. A second requirement is that, to be evolutionarily stable, solution mechanisms have to be functions of population growth, rather than of the state of the commons. This is a subtle point that became clear only as solution simulations based on "Conscience" and nonconsumable use values were investigated (Chapter VIII). $C(x)$ and $N(x)$ are functions of the state of the commons, whereas $\pi(T)$ is a function of demand. Demand is driven by population growth ${ }^{17}$, but the factors in the simulation that counteract demand are driven by the state of the commons. As the population grows, two things happen that make solutions more difficult: (1) the demand curve shifts out, away from the origin, meaning that the profit per unit harvested goes up for any given supply. The result is that Taking becomes increasingly attractive; (2) the state of the commons declines even faster because there are more people consuming and harvesting.

Social dilemmas happen because of the tenuousness of the connection between people's choices and the impacts of those choices: the true costs of an individual's actions are paid by others, whereas the individual gets the benefits. In these simulations, the benefits (i.e., profits) are a function of supply and demand, which are functions of population size - but the costs ("Conscience" and nonconsumable commons values) are in terms of the state of the commons. Benefits of Taking thus increase with

\footnotetext{
${ }^{17}$ As well as by aspirations, of course. If per capita consumption increases at the same time population increases, as it appears to be doing worldwide for most resources, the problem becomes even more difficult.
} 
population whereas costs of Taking do not. Any attempt to solve the TOC by resourcedriven solutions (i.e., any penalties or rewards that are functions of the state of the commons) will eventually be overtaken and overwhelmed by the effects of continued population growth.

To illustrate how a solution mechanism based on population size might work, a simulation was developed for an imaginary "free for all" fishery, where the penalty imposed on an illegal fisher who gets caught is simply the opposite of the equivalent profit function. The potential penalty thus increases with population, just as does demand. The solution is evolutionarily stable in the simulations for a growing population, because of its ability to ratchet up with demand. Unfortunately, it seems questionable that it would work in the real world because of enforcement problems and political difficulties, both of which would undoubtedly increase with population if demand does.

\section{Different contributions of game theory and decision theory}

Table IX summarizes the key differences in contributions to this work by the decision theory simulations and game theory analyses, and indicates that there are seven important aspects of the TOC that are revealed by only one of the approaches used: if only the game theory analysis had been used, the impacts of uncertainty, impacts of diversity on DMs' ability to adapt to change, and impacts of commons stochasticity would have been missed. If only the decision theoretical simulations had been used, the 
conflicts between individual and group "rationality", pareto-optimality of outcomes to the community, impacts of DM diversity on pareto-optimality of outcomes, and impacts of nonmarginal attributes would have been missed.

\begin{tabular}{|l|c|c|}
\hline $\begin{array}{l}\text { Aspect of TOC revealed (+) or not revealed (-) } \\
\text { by game theory analysis or decision theory } \\
\text { simulations }\end{array}$ & $\begin{array}{c}\text { Game } \\
\text { theory } \\
\text { analysis }\end{array}$ & $\begin{array}{c}\text { Decision } \\
\text { theory } \\
\text { simulations }\end{array}$ \\
\hline Net benefit of potential outcomes to individuals & + & + \\
\hline Pareto-optimality of outcomes to community & + & - \\
\hline Conflicts between individual and group "rationality" & + & - \\
\hline Tradeoffs among multiple attributes & + & + \\
\hline Impacts of uncertainty & - & + \\
\hline $\begin{array}{l}\text { Impacts of diversity on DMs' ability to adapt to } \\
\text { change }\end{array}$ & - & + \\
\hline $\begin{array}{l}\text { Impacts of DM diversity on pareto-optimality of } \\
\text { outcome }\end{array}$ & + & - \\
\hline Impacts of commons stochasticity & - & + \\
\hline Impacts of nonmarginal attribute $N(x)$ & + & - \\
\hline
\end{tabular}

Table IX. Contrasting contributions of the game-theoretical analysis and the decision-theorelical simulations. Aspects of the development of, and potential solutions to, the Tragedy of the Commons that were revealed by each type of analysis are indicated with a "+"; aspects that were not revealed are indicated with a "-".

Game theory had more to contribute to understanding the TOC than was initially expected, particularly because of the effects of the nonmarginal, nonconsumable value $N(x) . N(x)$ is key to the illustration of the conflicts between individual and group rationality, because without $N(x)$, the Prisoner's Dilemma cannot develop. Yet $N(x)$, being a nonmarginal attribute, is irrelevant to the decision trees. In prescriptive decision theory, the only attributes that matter are those that are affected by a DM's individual choice (an economist would say that decisions should be made "at 
the margin"), and so the nonconsumable value function $N(x)$ drops out of the decision trees. As far as any DM would be concerned, it would not matter if $N(x)$ were included in the decision tree or not.

If the decision were framed to include a total social welfare attribute, the pareto-optimality of outcomes could be revealed. However, decision trees are ordinarily focused on a particular decision-maker (or decision-making body), and so individual-vs-group conflicts tend to be invisible. Expected utility to the individual DM is the focus of a decision tree, whereas pareto-optimality of the outcome to all concerned is highlighted in game theory analyses.

In decision theory, considering payoffs to others is only useful insofar as it helps resolve uncertainties about what others might do. In game theory, payoffs to others are fundamental to the strategic aspects of games that involve cooperation as well as competition. A game theory matrix or graph of the PD makes it clear that if everyone chooses their dominant strategy ("always Take"), the payoff each receives will be less than each would have received if all had chosen oppositely: the dominant strategy results in a deficient outcome. This is invisible to decision theory.

Game theory analyses miss the fundamental impacts of uncertainty, adaptation, and stochasticity, that the decision theory based simulations enhance. Uncertainties about what others will do, and what the state of the commons will be, are well represented and quantified by decision trees, but uncertainty is only dealt with in game theory crudely, by making assumptions about payoffs and decision rules used by other 
players. Environmental stochasticity is mostly invisible to game theory, because game theory focuses on interactions among human players; Nature is at most a disturbance input and has no "strategic" role to play (games against Nature are generally better dealt with by decision theory because they are really only decisions under conditions of uncertainty). In contrast, with its greater power to quantify and represent uncertainty, the decision theory simulations incorporate, and illustrate the effects of, environmental stochasticity with ease.

Game theory and decision theory are both powerful tools, and both revealed the net benefit of potential outcomes and the tradeoffs among multiple attributes. Most important, though, the contributions of both turned out to be important to understanding this problem and its potential solutions. Decision theory seems to have the edge in incorporating uncertainty, and hence natural phenomena, into the analysis; but game theory seems to have the edge in explicating the human-interaction kinds of issues.

\section{RELATIVE IMPACTS AND INTERACTIONS OF VARIABLES}

Five factorial experiments were conducted in order to investigate the relative impacts of simulation variables (Chapter VII). The variables found to be significant in the factorial experiments were: the profit function parameter $k$ and "Conscience" function parameter $m$ ( $k$ and $m$ determine the functions' slopes and $y$-intercepts); 
commons stochasticity Stoch; and the evolutionary diversity control parameters TSProb and $m r$. The probability estimates and outcome tables used in the EMAV decision trees were varied to represent populations that were whole relatively pessimistic or optimistic, but were not found to be statistically significant in any experiments. $N(x)$ has no impact on people's choices because people get the $N(x)$ payoff regardless of what they as individuals do; it was therefore not included in the experiments.

The most important single parameter was the mutation rate $m r$. The mutation rate is the small probability (e.g., 0.001 ) that one of the variables in a DM's decision tree will be flipped to its opposite value. For example, a mutation in the optimism "gene" may convert an optimist to a pessimist; a mutation in one of the DM Type genes will turn a hard core extractor DM into a hard core preservationist. The mutation rate thus constantly (re)introduces variety into the population. The higher $m r$ is, the more "irrational" DMs there will be in any iteration.

The profit function parameter $k$, commons stochasticity Stoch, and progenitor diversity parameter TSProb, were all about equally important as main effects, and all were also involved in significant two-factor interactions. TSProb is important to population diversity because it impacts the probability that relatively more successful genotypes will be represented in successive iterations; if TSProb is low, then the population will have more nonconformists. The "Conscience" function parameter was involved in two-factor interactions and a three-factor interaction, but its significance as a main effect was hidden by the nature of the interactions it was involved in. 
The profit function parameter and "Conscience" function parameter are important because they represent the tradeoffs people make between greed and guilt. Hardin's herdsmen only considered profit, but "Conscience" is arguably an important part of human decision-making. The simple function that represents "Conscience" in this work is very crude, at best, but the impact it had on the outcome was significant, not only because it was necessary for most solutions, but also because only if $C(x)$ is included can games without the dominant but non-pareto-optimal strategy of the PD develop. Unlike the $\mathrm{PD}$, which is the only game normally discussed in the context of the $\mathrm{TOC}^{18}$, Hero and Benevolent Chicken are representative of some of the more appealing aspects of human decision-making.

Furthermore, $C(x)$ is the only mechanism in the model that works to offset greed. Most of the simulated solutions depend on either decreasing the profit function parameter or increasing the "Conscience" function parameter, and it was expected that these two parameters would be the most important in the experiments. The importance of the evolutionary diversity control parameters $m r$ and TSProb was a surprise.

\section{SOLVING THE TRAGEDY OF THE COMMONS}

The solutions discussed in the literature all have to do with resolving conflicts between individual and group interests: in effect, changing the payoffs or changing the

\footnotetext{
${ }^{18}$ The only reference to Chicken with respect to the TOC that was found in the literature search was Hamburger's (1973) article.
} 
rules. The means proposed to do this were initially lumped into six, somewhat overlapping categories: technology, community identification, financial incentives, property rights, regulatory control, and population control. A seventh category, nonconsumable value, was added in the game theory analyses.

The computer model was used to crudely simulate and explore such solutions. Technological solutions were simulated by changing the profit function to represent decreasing demand via increased efficiency of use or development of substitutes, and by changing the regeneration rate function to represent increasing supply. Community identification and moral concerns were simulated by increasing the "Conscience" function parameter. Increasing penple's appreciation of nonconsumable values such as aesthetics was simulated by adding a nonconsumable value function to the decision trees for some of the DMs. Financial incentives were examined via a "thought experiment" based on the results of varying the profit and "Conscience" functions. Property rights solutions were not simulated because the computer model was based on an assumption of public access to the commons, and property rights solutions require essentially turning the commons over to private ownership or at least private control. Modifying the computer model to include property rights-based solutions proved to be beyond the scope of this work, although investigating such solutions appears to be a promising line of further work. Population control was not simulated as a separate category, but was instead included in each of the solution simulations (as well as in the preliminary development work of Phase I). This was justified because the question 
being investigated was whether any solution could be shown to work with population growth, and thus each solution simulation had to "stand up" to the test of population growth.

The main finding was that solutions based on increasing commons productivity or efficiency of use, or increasing the relative impacts of "Conscience" or nonconsumable resource value, or imposing rewards and penalties, could be invented for any fixed population size; but to work for growing populations, solutions have to keep up with population growth. For example, in order to provide enough of a solution to allow about a threefold increase in population, the relative importance of "Conscience" had to increase about tenfold in the simulations.

Similarly, solutions based on technology had to provide about a tenfold decrease in the slope of the demand curve, or a ninefold increase in the commons regeneration rate constant, to support a threefold increase in population.

The goal of the simulations was not to determine whether any particular solution would work, but rather to investigate Hardin's contention that no solutions would work unless population growth is stopped. This work supported Hardin's contention: for any simulated solution to continue working for a growing population, its effectiveness had to increase at least as fast as population increased. There is no evidence in the real world that any of the solution mechanisms has become more effective as population has grown, and in fact the evidence has been that population growth makes them all more problematic: the technological miracles of the green 
revolution have become subject to declining marginal returns, while population continues to grow exponentially; the power of community identification and moral concerns becomes weaker, not stronger, as population grows; as population grows, solutions based on imposing property rights have failed not only in historical grazing commonses throughout Africa but also in fishing commonses around the world; and solutions based on financial incentives have shown themselves to be vulnerable to problems with enforcement and political pressures that increase with increasing population.

This gloomy conclusion is hardly surprising, and it is worth noting that in the real world, the effectiveness of any solution actually has to increase at least as fast as consumption increases. As long as per-capita consumption continues to increase even faster than population growth, the challenge for any solution is even tougher than Hardin suggested.

\section{SUGGESTIONS FOR FURTHER WORK}

This model is a first effort to extend Hardin's simple metaphor to something that can be used on a computer to help think through and perhaps to solve the tragedies of natural resource destruction. It produced a number of interesting and potentially powerful insights, not the least of which was that Hardin was right, even if attributes that Hardin dismissed are included, and even if an overly optimistic model of human 
decision-making is assumed. It also suggested a number of lines of promising further work.

The key elements in the model are represented here with very simple mathematical functions: "Conscience" and profit functions are continuous, linear, functions of single variables; all the elements of uncertainty are represented by one "gene" in the computer code; human population growth is modeled as a deterministic, apparently infinitely growing exponential function independent of anything in the rest of the model; commons regeneration is modeled with an introductory biology textbook's single-variable logistic function; commons stochasticity is added via a normal probability distribution. The human tendency to discount the future is not represented explicitly and is only vaguely implied in the "Conscience" function, and there is no real way to simulate private property issues and the conflicts between the profits of "upstream" extractors and costs paid by their "downstream" victims.

Each of these simplifications is nonetheless a move towards reality from Hardin's simple assumption of a " +1 " benefit for Taking and " 0 " benefit for Refraining, with no accounting for potential impacts of "Conscience", uncertainty, environmental stochasticity, or discounting, all of which are widely acknowledged to be important aspects of human decision-making. However, since all these elements turned out to have implications regarding at least buying time that appears to be needed, if not solving the TOC over the long run, they all inspire ideas for further work.

Because this is almost entirely a theoretical model, the most important "next 
step" in this line of work would have to be empirical. In particular, the "Conscience" function suggests the most immediately interesting empirical investigation: that is, to derive an empirically-based function (or functions) that represents moral considerations. Two approaches come immediately to mind: analyzing money spent on conservation activity, in terms of perceived condition of the resource (e.g., amount of money spent on activities to protect Idaho salmon/stocks perceived as endangered, threatened, sensitive, and healthy); and using decision-analytic value function elicitation methods to derive value functions for "Conscience" (perhaps as more than one attribute) of a variety of kinds of people. Each approach would be interesting alone, but a comparison of the two would probably also reveal some significant issues about the relationship between environmental law and social values.

The profit function could also be made considerably more complex and realistic, by, for example, explicitly including extraction costs and discount rates, perhaps based on a real resource. Extraction costs (and perceptions of "true" extraction costs), as well as discount rates, could certainly be represented by "genes" in the algorithm. DMs who ignore global and future ("true") extraction costs (i.e., externalities) and discount the future would be more likely to Take than those who do not, resulting in higher shortterm payoffs but faster depletion of the commons. If time lags were included in the feedback loops, actual long-term payoffs could be used in the selection mechanism, perhaps to illustrate how longer-term decision frames could help save the commons.

Other additions or modifications to the simulation that might be interesting but 
seem less pressing are: modifying the model to represent private property issues such as the impacts of externalities on both the DMs and the stakeholders; incorporating the nonconsumable value attribute function $N(x)$ as a variable in the genotypes; investigating the impacts of different assumptions about how population grows (e.g., not exponentially but logistically, or tying population growth to the commons in a Lottka-Volterra kind of model); and adding "Conscience" to the regulatory control solution simulations.

\section{CONCLUSION}

The conditions under which we evolved have changed, if for no other reason than because there are now so many more of us. Evolution favors having many offspring, but survival of the species now appears to favor having few. Hardin (1968) says that controlling population growth is the only solution to the most important problem in the world, and that the problem of controlling population growth is an even more difficult commons problem than is grazing or fishing. Since population growth drives commons problems such as overgrazing or overfishing, it is an even more important commons problem itself.

It is important to think about not only what kind of world we can survive in, but what kind of world we want to survive in. Hardin (1968) points out that we could maximize population, but only if we minimize the quality of our lives. A great deal of 
research has recently been conducted to determine the world's human carrying capacity, with the odd result that the variance of the estimates has actually increased, rather than converging towards a probably true mean as one might expect (Cohen, 1995); yet it should not be difficult to see how much simpler these problems would be if there were fewer, not more, people than there are now. It is inconceivable that anyone's quality of life will be improved with the doubling of the world's population predicted in the next 40 years.

It may even be that there have been too many people on the planet for some time. About four generations ago, when there were about one-sixteenth as many people as there are now, John Stewart Mill warned of the kind of world we were moving toward, and his warning is at least as relevant today as it was in our great-greatgrandparent's day (Mill, 1857, 320-326):

It is not good for a man to be kept perforce at all times in the presence of his species....Nor is there much satisfaction in contemplating the world with nothing left to the spontaneous activity of nature; with every rood of land brought into cultivation, which is capable of growing food for human beings; every flowery waste or natural pasture plowed up, all quadrupeds or birds which are not domesticated for man's use exterminated as his rivals for food, every hedgerow or superfluous tree rooted out, and scarcely a place left where a wild shrub or flower could grow without being eradicated as a weed in the name of improved agriculture.

If Mill and Hardin are right, then what should be done?

The implications of this research related to that question are that:

- Solving the TOC requires enough diversity of beliefs and values that the 
population can respond in time to changes. Such diversity is maintained in political and social systems that tolerate and nurture contrary viewpoints, and are willing to invest in education and basic research.

- Stochasticity of the commons must be low enough, and/or forecasting accurate enough, to allow planning and decision-making to be effective. This is partly a technological challenge, but it is also a conservation challenge: the stability of natural systems is a strong function of their biodiversity, and biodiversity is inversely related to human impacts. As population grows, biodiversity decreases, and stochasticity increases.

- There must be enough of a benefit for Refraining or cost for Taking, that enough people will choose to Refrain to conserve important commonses. The most common mechanisms for providing a benefit for Refraining or penalty for Taking are social and economic. People in small, closely-knit communities share and conserve because of group pressure; in large, unconnected communities, people have to rely on more complex political systems to impose bonuses and penalties that are part of what Hardin called "mutual coercion, mutually agreed on."

- Demand must be elastic enough that people feel they actually have a choice. There needs to be an abundance of the resource or acceptable substitutes available, because demand is increasingly inelastic as people feel increasingly desperate. In the limit, abundance can be increased only by 
improving efficiency of use. If the rate of consumption of natural resources continues to increase, then resources will need to be used only for the highest-priority uses, and reaching world-wide consensus on prioritization (particularly between rich and poor countries) would probably be impossible.

- These measures (maintaining diversity, reducing stochasticity, changing the payoffs so people can and will Refrain) are merely stopgap measures that will fail if population continues to grow. It becomes more difficult, but more important, to achieve these measures as population grows. Controlling population growth may be viewed as a social dilemma in its own right.

These are not revolutionary findings, and if it were not for the heated debates in professional journals of economics, ecology, biology, and human ecology, one would think they were more or less obvious. Are they hopeful findings? There is little indication in human history, particularly recently, that any of these requirements for solving the Tragedy of the Commons is likely to be met anytime soon. Denial, as Hardin has often said, is one of the most powerful human tendencies because it has been selected for over millennia of evolution: there is little benefit to any of us as individuals in sacrificing our own desires for the sake of other people's children's futures. 


\section{REFERENCES}

Agarwall, M., Shukla, A., and Pal, V. N. 1993. Grazing of forested grassland and its conservation. Ecological Modelling. 69:57-62.

Alexander, M. 1981. Why microbial predators and parasites do not eliminate their prey and hosts. Anmual Review of Microbiology. 35:113-133.

Allan, J. D., and Flecker, A. S. 1993. Biodiversity conservation in running waters. BioScience. 43(1):32-43.

Ashby, W. R. 1956. An Introduction to Cybernetics. New York: J. Wiley.

Axelrod, R. 1984. The Evolution of Cooperation. USA: BasicBooks.

Axelrod, R. 1987. The Evolution of Strategies in the Iterated Prisoner's Dilemma. London: Pittman.

Ball, G. F., and Gimblett, R. 1992. Spatial dynamic and emergent hierarchies simulation and assessment system. Ecological Modelling. 62:107-121.

Bassett, T. J. 1988. The political ecology of peasant-herder conflicts in the northern Ivory Coast. Ammals of the Association of American Geography. 78(3): 453472 .

Bedau, M. A., and Packard, N. H. 1990. Measurement of Evolutionary Activity, Teleology, and Life. In Artificial Life II, X, ed. C. G. Langton, C. Taylor, J. D. Farmer, and S. Rasmussen, pp. 431-462. Santa Fe, NM: Addison-Wesley Publishing Co.

Belyaev, V. I., and Khudoshina, M. Y. 1992. Logical-information modelling of ecosystems. Ecological Modelling. 60:119-138.

Bencherifa, A., and Johnson, D. L. 1991. Changing resource management strategies and their environmental impacts in the middle Altlas mountains of Morocco. Moumtain Research and Development. 11(3):183-194.

Berkes, F., Feeny, D., McCay, B. J., and Acheson, J. M. 1989. The benefits of the commons. Nature. 340:91-93.

Boyle, K. J., and Bishop, R. C. 1987. Valuing wildlife in benefit-cost analyses: a case study involving endangered species. Water Resources Research. 23(5):943-950.

Brown, L. R., Lenssen, N., and Kane, H. 1995. Vital Signs 1995: The Trends That Are Shaping Our Future. New York: W.W. Norton and Company.

Cass, R. C., and Edney, J. J. 1978. The commons dilemma: a simulation testing the effects of resource visibility and territorial division. Human Ecology. 6(4):371386. 
Cohen, J. E. 1995. Population growth and earth's human carrying capacity. Science. 269:341-346.

Collins, D., and Barkdull, J. 1995. Capitalism, environmentalism, and mediating structures: from Adam Smith to stakeholder panels. Environmental Ethics. 17:227-244.

Costanza, R. 1987. Social traps and environmental policy: why do problems persist when there are technical solutions available? BioScience. 37(6):407-412.

Costanza, R., and Daly, H. E. 1992. Natural capital and sustainable development. Conservation Biology. 6(1):37-46.

Daily, G. C., and Ehrlich, P. R. 1992. Population, sustainability, and earth's carrying capacity. BioScience. 42:761-771.

Daly, H. E. 1980. Introduction to the steady-state economy. In Economics, Ecology, Ethics, ed. H. E. Daly, pp. 1-32. San Francisco: W.H. Freeman and Co.

Daly, H. E. 1995. Reply to Mark Sagoff's "Carrying capacity and ecological economics". BioScience. 45(9):621-624.

Dawes, R. M. 1980. Social dilemmas. Anmual Review of Psychology. 31:169-93.

Dawkins, R. 1978. The Selfish Gene. New York: Oxford University Press.

Dietz, T., and Burns, T. R. 1992. Human agency and the evolutionary dynamics of culture. Acta Sociologica. 35:187-200.

Dietz, T., and Rosa, E. A. 1994. Rethinking the environmental impacts of population, affluence and technology. Human Ecology Review. Summer/Autumn. 1:277300.

Dowie, M. 1995. Losing Ground. Cambridge, MA: MIT Press.

Duncan, L. 1995. Closed competition: fish quotas in New Zealand. The Ecologist. 25 (2/3):97-104.

Editors. 1995. Overfishing: its causes and consequences. The Ecologist. 25(2/3):4245.

Edney, J. J., and Harper, C. S. 1978. The effects of information in a resource management problem: a social trap analog. Human Ecology. 6(4):387-395.

Edwards, P. N. 1995. Global comprehensive models in politics and policymaking. Climatic Change. Sept. 1995.

Ehrenfeld, D. 1981. The Arrogance of Humanism. Oxford: Oxford University Press.

Etzioni, A. 1988. The Moral Dimension: Toward a New Economics. New York: The Free Press. 
Fahrig, L. 1991. Simulation methods for developing general landscape-level hypotheses of single-species dynamics. In Quantitative Methods in Landscape Ecology: the Analysis and Interpretation of Landscape Heterogeneity, ed. M. G. Turner and R. H. Gardner, pp. 417-442. New York: Springer-Verlag.

Fairlie, S., Hagler, M., and O'Riordan, B. 1995. The politics of overfishing. The Ecologist. 25(2/3):46-73.

Fiering, M. B., and Holling, C. S. 1974. Management and standards for perturbed ccosystems. Agro-Ecosystems. 1:301-321.

Fife, D. 1971. Killing the goose. In Managing the Commons, ed. Hardin, G. and J. Baden, pp. 76-81. San Francisco: W.H. Freeman and Company.

Fowler, C., and Mooney, P. 1990. Shattering: Food, Politics, and the Loss of Genetic Diversity. Tucson: University of Arizona Press.

Fujita, R., and Hopkins, D. 1995. Market theory can help solve overfishing. The Oregonian, Sept. 20, 1995, p. B7.

Georgescu-Roegen, N. 1971. The Entropy Law and the Economic Process.

Cambridge, MA: Harvard University Press.

Gillett, P. 1981. Calculus and Analytical Geometry. Lexington, MA: D.C. Heath and Company.

Goldberg, D. E. 1989. Genetic Algorithms. Reading, MA: Addison-Wesley Publishing Co. Inc.

Grant, W. E. 1986. Systems Analysis and Simulation in Wildlife and Fisheries Sciences. New York: John Wiley and Sons.

Griffin, D. R. 1992. Animal Minds. Chicago: University of Chicago Press.

Gwartney, J. D., and Stroup, R. 1980. Economics: Private and Public Choice (2nd ed.). New York: Academic Press.

Hamburger, H. 1973. N-person prisoner's dilemma. Journal of Mathematical Sociology. 3:27-48.

Hamburger, H. 1979. Games as Models of Social Phenomena. New York: W.H. Freeman and Company.

Hardin, G. 1968. The tragedy of the commons. In Managing the Commons, ed. G. Hardin and J. Baden, pp. 16-30. New York: W.H. Freeman and Company.

Hardin, G. 1971. Ethical implications of carrying capacity. In Managing the Commons, ed. G. Hardin and J. Baden, pp. 112-125. San Francisco: W.H. Freeman and Company. 
Hardin, G. 1977. Denial and disguise. In Managing the Commons, ed. G. Hardin and J. Baden, pp. 45-52. New York: W. H. Freeman and Company.

Hardin, G. 1993. Living within Limits: Ecology, Economics, and Population Taboos. New York: Oxford University Press.

Hardin, G. 1994. A commons error (letter to the editor). Scientific American. 270(5): 10 .

Hardin, G., and Baden, J. 1977. Managing the Commons. New York: W.H. Freeman and $\mathrm{Co}$.

Harrison, P. 1994. Sex and the single planet: need, greed, and earthly limits. Human Ecology Review. 1(2):229-233.

Heinen, J., and Low, R. 1992. Human behavioral ecology and environmental conservation. Envirommental Conservation. 19(2):105-116.

Hillis, W. D. 1990. Co-evolving parasites improve simulated evolution as an optimization procedure. In Artificial Life II, X, ed. C. Langton, C. Taylor, J. Farmer, and S. Rasmussen, pp. 313-324. Santa Fe, NM: Addison-Wesley Publishing Co.

Hobbes, T. 1649. In Leviathan (1947), ed. M. Oakeshott, Chapter 13. New York: Oxford University Press.

Hogarth, W. L., Norbury, J., Cunning, I., and Sommers, K. 1992. Stability of a predator-prey model with harvesting. Ecological Modelling. 62:83-106.

Holland, J. H. 1975. Adaptation in Natural and Artificial Systems. Ann Arbor: University of Michigan Press.

Holland, J. H. 1992. Genetic algorithms. Scientific American. 267(1):66-72.

Holling, C. S. 1969. Stability in ecological and social systems. In Brookhaven Symposia in Biology Number 22, pp. 128-141. Brookhaven National Laboratory: U.S. Department of Commerce.

Holling, C. S. 1976. Resilience and stability of ecosystems. In Evolution and Consciousness, pp. 73-92. Reading, MA: Addison-Wesley Publishing Co.

Holling, C. S. 1978. Adaptive Environmental Assessment and Management. Chichester: John Wiley and Sons.

Holling, C. S., and Clark, W. C. 1975. Notes towards a science of ecological management. In First International Congress of Ecology, ed. W. H. van Dobben and R. H. Lowe-McConnell, pp. 247-251. The Hague, the Netherlands: Dr W. Junk B.V. Publishers. 
Huntington, C. 1994. Chair, Natural Production Committee, Oregon chapter of the American Fisheries Society. Personal communication.

Huntington, C., Nehlsen, W., and Bowers, J. 1996. A survey of healthy native stocks of anadromous salmonids in the Pacific Northwest and California. Fisheries. In press.

Jeffers, J. N. R. 1988. Practitioner's Handbook on the Modelling of Dynamic Change in Ecosystems. Chichester: John Wiley and Sons.

Jeffreys, K. 1993. Property rights and marine resources. Fisheries. 18(9): 26-28.

Keeney, R. 1992. Value-Focused Thinking. London: Harvard University Press.

Keeney, R. L., and Raiffa, H. 1976. Decisions with Multiple Objectives: Preferences and Value Tradeoffs. New York: John Wiley and Sons.

Kent, G. 1995. "Fish for the poor": competing with chickens. The Ecologist. 25(2/3):48.

Kompaore, S. 1989. Women as managers of village water resources. Natural Resources Forum, Nov. 1989. 319-321.

Lindgren, K. 1990. Evolutionary phenomena in simple dynamics. In Artificial Life II, X, ed. C. G. Langton, C. Taylor, J. D. Farmer, and S. Rasmussen, pp. 295312. Santa Fe, NM: Addison-Wesley Publishing Co.

Low, B. S., and Heinen, J. T. 1993. Population, resources, and environment: implications of human behavioral ecology for conservation. Population and Environment. 15(1):7-41.

Ludwig, D., Hilborn, R., and Walters, C. 1993. Uncertainty, resource exploitation, and conservation: lessons from history. Science. 260(2):17, 36.

MacCrimmon, K. R., and Messick, D. M. 1976. A framework for social motives. Behavioral Science. 21:86-100.

Mace, P. M. 1993. Will private owners practice prudent resource management? Fisheries. 18(9):29-31.

Mathews, D. R. 1995. Commons versus open access: the Canadian experience. The Ecologist. 25(2/3):86-96.

McCay, B. J. 1978. Systems ecology, people ecology, and the anthropology of fishing communities. Human Ecology. 6(4):397-422.

Meffe, G. K., and Ehrlich, A. H. 1993. Human population control: the missing agenda. Conservation Biology. 7(1):1-3.

Mill, J. S. 1857. Principles of Political Economy. London: John W. Parker. 
Miller, J. H. 1989. The co-evolution of automata in the repeated Prisoner's Dilemma No. 89-003. Santa Fe Institute.

Monbiot, G. 1994. The tragedy of enclosure. Scientific American. 270(1):159.

Morowitz, H. J. 1991. Balancing species preservation and economic considerations. Science. 253:752-754.

Muhsam, H. V. 1977. An algebraic theory of the commons. In Managing the Commons, ed. G. Hardin and J. Baden, pp. 34-37. New York: W. H. Freeman.

Ntiamoa-Baidu, Y. 1991. Conservation of coastal lagoons in Ghana: the traditional approach. Landscape and Urban Plaming. 20: 41-46.

Odum, E. P. 1969. The strategy of ecosystem development. Science. 164:262-270.

Odum, E. P. 1977. The emergence of ecology as a new integrative discipline. Science. 195(4284):1289-1293.

Ostrom, E. 1990. Governing the Commons: the Evolution of Institutions for Collective Action. Cambridge, England: Cambridge University Press.

Patten, B. C., and Odum, E. P. 1981. The cybernetic nature of ecosystems. The American Naturalist. 118:886-895.

Pearce, D., and Moran, D. 1994. The Economic Value of Biodiversity. London: Earthscan Publications Ltd.

Pimm, S. L., Russell, G. J., Gittleman, J. L., and Brooks, T. M. 1995. The future of biodiv'ersity. Science. 209:347-350.

Prugh, T., Costanza, R.t, Cumberland, J. H., Daly, H., Goodland, R., and Norgaard, Richard B. 1995. Natural Capital and Human Economic Survival. Solomons, MD: International Society for Ecological Economics Press.

Rapoport, A. 1967. Escape from paradox. Scientific American. 217(1):50-59.

Ridley, M. 1993. The Red Queen: Sex and the Evolution of Human Nature (1 ed.). New York: Macmillan.

Riolo, R. L. 1992. Survival of the fittest bits. Scientific American. 267(1):114-116.

Russo, J. E., and Shoemaker, P. J. H. 1989. Decision Traps: the Ten barriers to Brilliant Decision-making and How to Overcome Them. New York: Fireside Books.

Sagoff, M. 1995. Carrying capacity and ecological economics. BioScience. 45(9):610620.

Shubik, M. 1970. Game theory, behavior, and the paradox of the prisoner's dilemma: three solutions. Journal of Conflict Resolution. 14(2):181-193. 
Simon, J., and Wildavsky, A. 1993. Facts, not species, are periled. New York Times, May 13, 1993.

Smith, A. 1776. The Wealth of Nations. New York: reprint, Modern Library, 1937.

Stern, P. C., Dietz, T., and Black, J. S. 1985-86. Support for environmental protection: the role of moral norms. Population and Environment. 8(3 and 4):204-222.

Stott, P. 1991. Recent trends in the ecology and management of the world's savanna formations. Progress in Physical Geography. 15(1):18-28.

SYSTAT 1992. SYSTAT for Windows. Evanston, IL: SYSTAT, Inc.

Trout, P. A. 1995. Book review: Garrett Hardin (1993) Living Within Limits: Ecology, Economics, and Population Taboos. Environmental Ethics. 17:331-336.

von Winterfeldt, D., and Edwards, W. 1986. Decision Analysis and Behavioral Research. Cambridge: Cambridge University Press.

Vrijenhoek, R. C. 1985. Animal population genetics and disturbance: the effects of local extinctions and recolonizations on heterozygosity and fitness. In The Ecology of Natural Disturbance and Patch Dynamics, ed. S. T. A. Pickett and P. S. White, pp. 265-282. Orlando: Academic Press, Inc.

Weiss, E. B. 1990. In fairness to future generations. Enviromment'. 32(3):7-31.

White, S.D. 1995. The rationale for biological conservation: a reply to Brussard. Society for Conservation Biology Newsletter. 2(2):2.

Wilson, E. O. 1984. The Biophilia Hypothesis. Cambridge: Harvard University Press.

Wilson, E. O. 1992. The Diversity of Life. Cambridge: Harvard University Press.

Wilson, E. O. 1993. Mass extinctions grow. New York Times, May 25, 1993. Letters to the editor. 


\section{A P P E N D I X}

\section{COMPUTER CODE}




\section{$1^{\text {* }}$ GENETIC ALGORITHM PROGRAMI, FROM THE DISSERTATION OF GRETCHEN OOSTERHOUT, 1996. */}

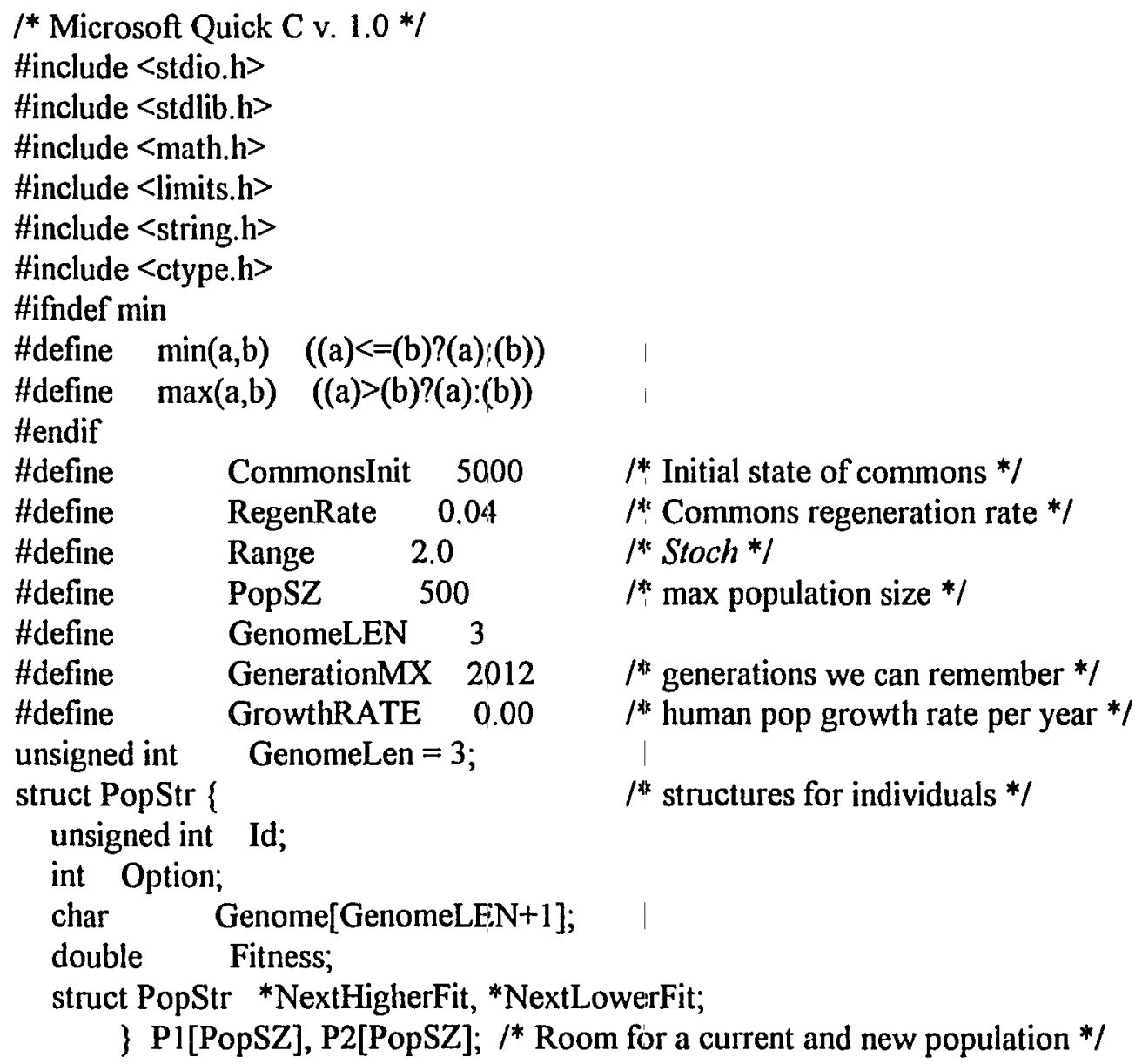




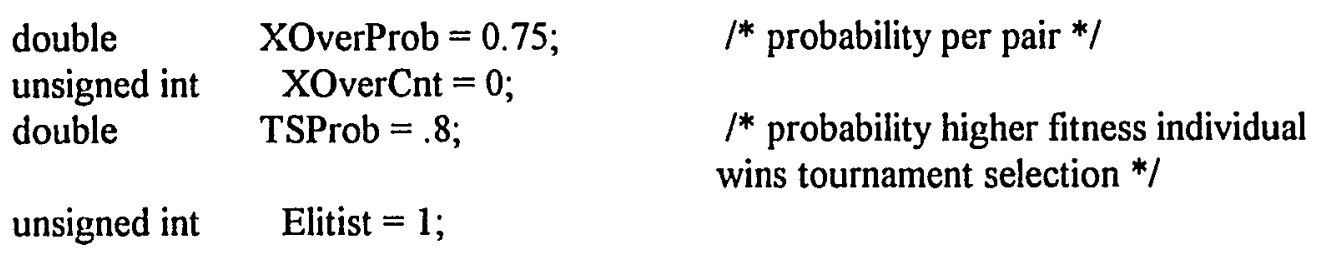

\section{/* DEFINITIONS RELATED TO THE FITNESS FUNCTIONS *}

/* float FitnessOfString; now a variable instead of a function $2 / 14 / 95 *$ /

double BinaryCharToDouble ( register char *Buff, register int $\mathrm{L}$ );

double BinaryCharToDoubleX( register char *Buff, register int $\mathrm{L}$ );

double $\operatorname{Maxf}=-1$;

double AveFitness[GenerationMX], BestFitness[GenerationMX];

\section{/*VARIABLES AND ROUTINES TO GENERATE RANDOM NUMBERS */}

\#define random() rand()

\#define URand01 ( (double) random()/(double) INT_MAX)

\#define RandStateSZ $256 /{ }^{*}$ for calls to random() in Ultrix lib */

char *RandState[RandStateSZ]; $/ *$ store state used by random ()$* /$

unsigned int RandSeed $=3787 ; \quad{ }^{*} 103$ user settable seed to get different sequences *I

\#define initstate( RandSeed, RandState, RandStateSZ) srand( RandSeed );

control pop. display format and frequency, and debugging messages */

unsigned int DisplayPopInt $=3000 ; / *$ If not 0 , display pop every $n$ generations */

unsigned int DisplayPopFmt $=0 ; / *$ default format for pop display $* /$

\section{/*FORWARD DECLARATIONS */}

void Restart(), EvaluatePop(), GenerateRandomPop(), PrintPop();

void Copylndiv(), TournamentSelection(), ModifyPopulation(), AddNew();

char * readline ( char *S, unsigned int L, FILE *F );

char ${ }^{*}$ striptoken( char *S, char *Token, unsigned int L, char *Breaks );

\section{/*MISC. VARIABLES AND FUNCTION PROTOTYPES*/}

FILE *fp, *fp2; $\quad$ /* file pointers */

double Sum, Delta, StDev; $\quad / *$ for standard dev of generation's fitness $* /$

char static MaxGenome[GenomeLEN+1]; $\quad I^{*}$ Genome of BestIndiv */

double xmax; $\quad l$ for BinaryCharToDouble */

float Commons;

float Takers; 
float TotalCommons, AveCommons, TotalTakers, AveTakers, GenCount;

float Regen;

float $\mathrm{z}$;

float StochReg;

void CharTolnt(char Charray[4], int Intarray[3]);

void Decoder(char Genotype[4], float w[3]);

int FindChoice(char geno[4]);

float Payoff(float NTakers, float State, float $* k$, float *d, int Choice);

float gasdev();

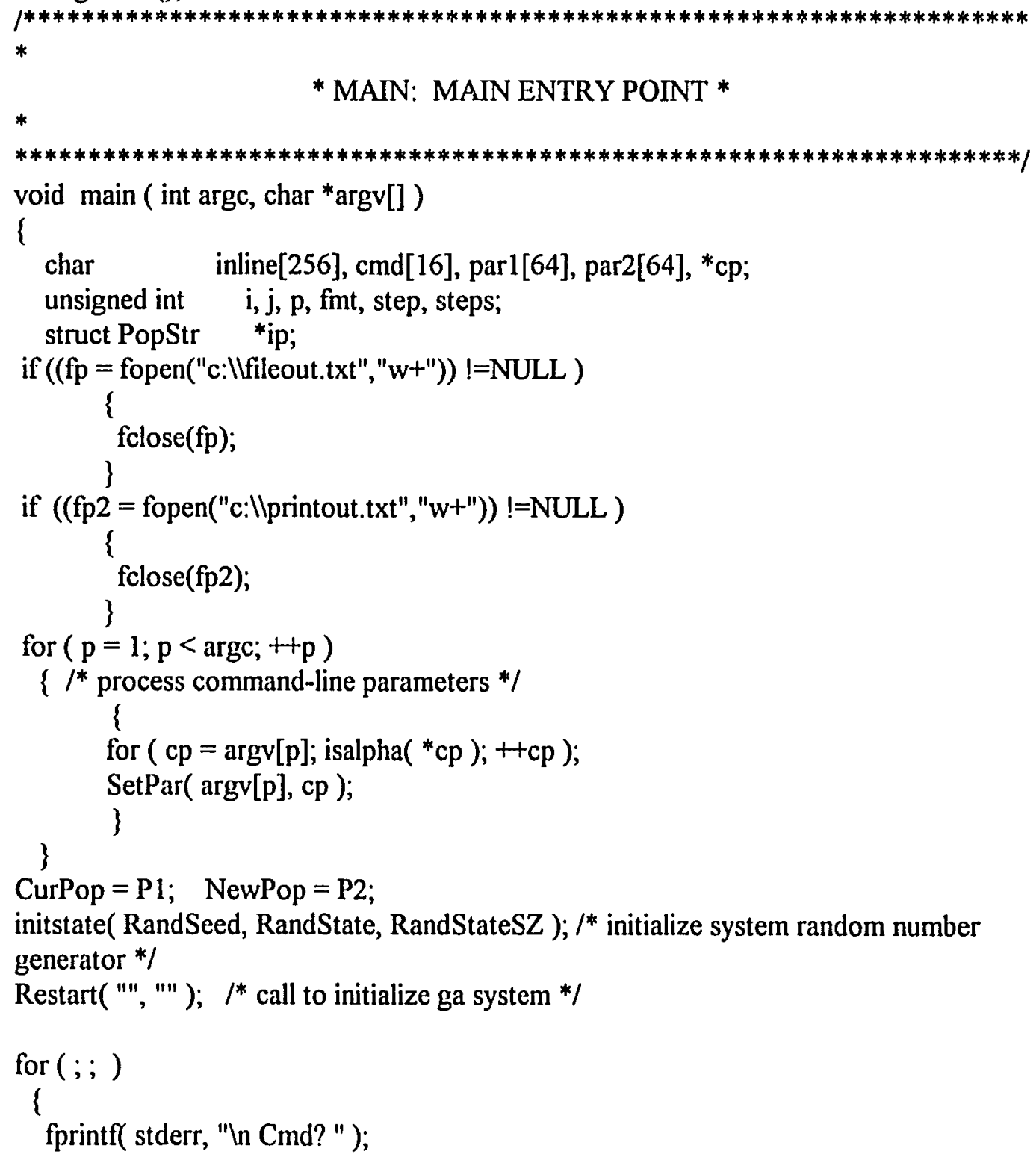


$\mathrm{cp}=$ readline( inline, sizeof(inline), stdin ); /* read command */

$\mathrm{cp}=$ striptoken ( $\mathrm{cp}, \mathrm{cmd}$, sizeof(cmd), " " ); $/ *$ get command into cmd, any par starts at $* \mathrm{cp} *$ /

if ( strncmp ( cmd, "q", 4) $=0 \|$ strncmp( cmd, "ex", 4) $=0$

\| strncmp ( cmd, "EX", 4)=0)

\{

break;

\}

else if ( strncmp( cmd, "di", 2) $=0$ )

\{

cp = striptoken $(\mathrm{cp}$, parl, sizeof(par1), ", ");

/* split into two paramters *I

$\mathrm{cp}=$ striptoken( $\mathrm{cp}$, par2, sizeof(par2), " " );

if ( $\operatorname{strcmp}($ par1, "pop" ) $=0$ )

\{

if ( *par2 = ' 10 ' ) fmt = DisplayPopFmt;

else $\mathrm{fmt}=$ atoi $(\operatorname{par} 2)$;

PrintPop( CurPop, fmt );

\}

else

\{

Display ( par1 );

\}

$/^{*}$ end else if $* /$

else if ( strncmp( cmd, "set", 3 ) $=0$ )

\{

$\mathrm{cp}=$ striptoken $(\mathrm{cp}$, parl, sizeof(parl $), "=")$;

$\mathrm{cp}=\operatorname{striptoken}(\mathrm{cp}$, par2, sizeof(par2), " " );

\}

SetPar( par1, par2);

else if ( strncmp( cmd, "res", 3 ) $=0$ )

\{

$\mathrm{cp}=\operatorname{striptoken}(\mathrm{cp}$, parl, sizeof(parl), ", " );

$\mathrm{cp}=\operatorname{striptoken}(\mathrm{cp}$, par2, sizeof(par2), " " );

\}

Restart( par1, par2 );

else if ( strncmp( cmd, "ep", 2 ) $=0$ )

\{

EvaluatePop( CurPop );

)

else if ( strncmp( cmd, "d2b", 3 ) ==0) 


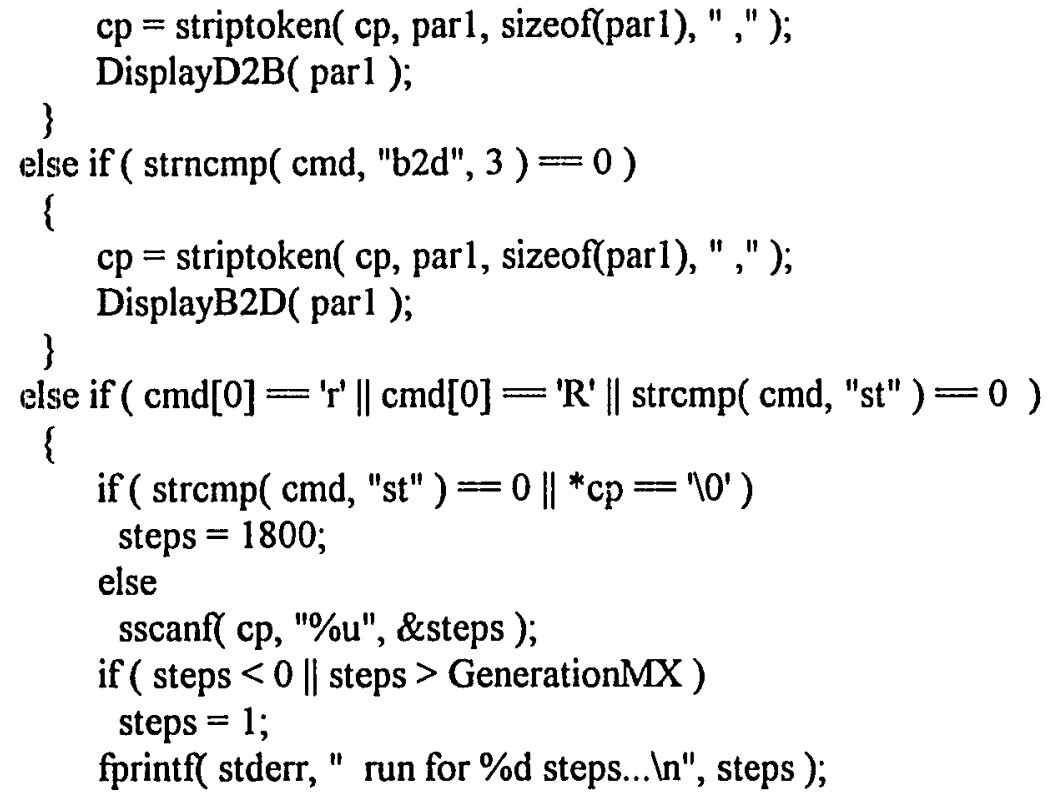




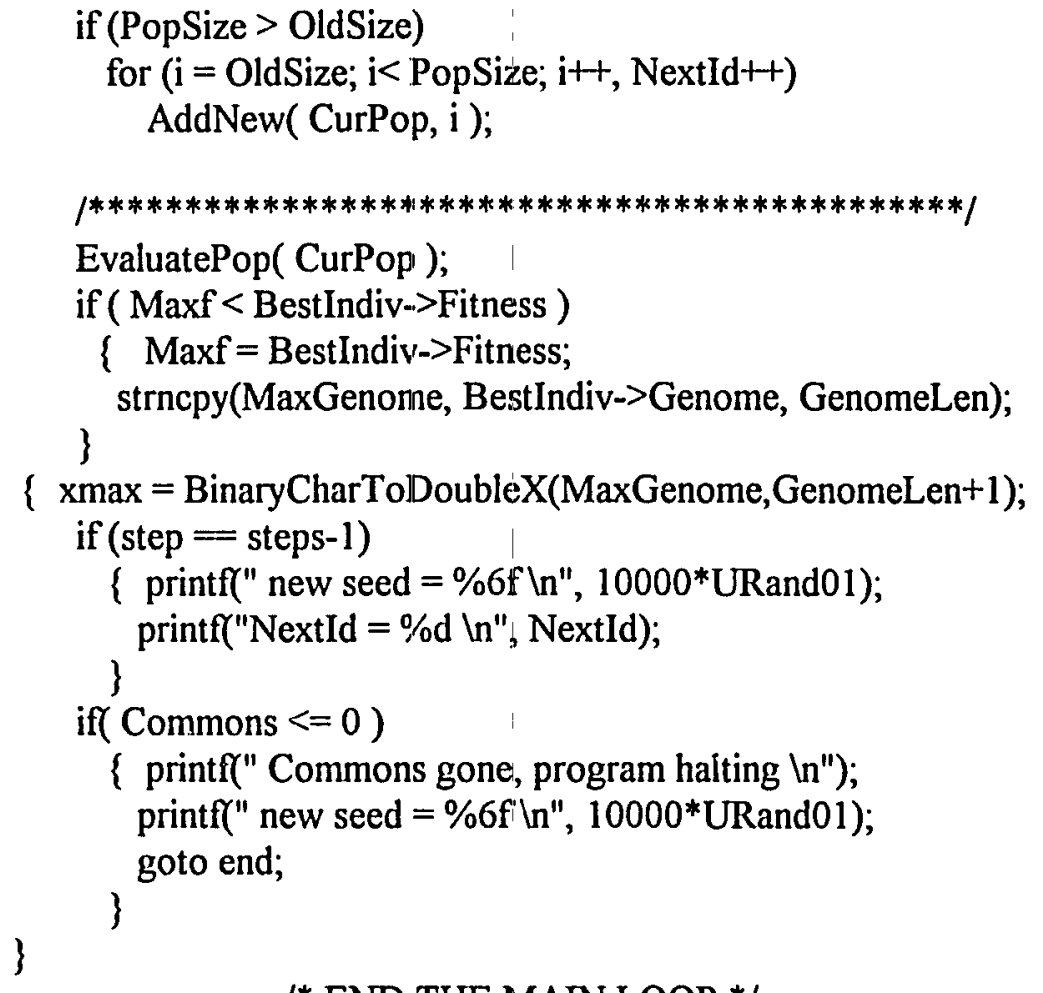

/* END THE MAIN LOOP */

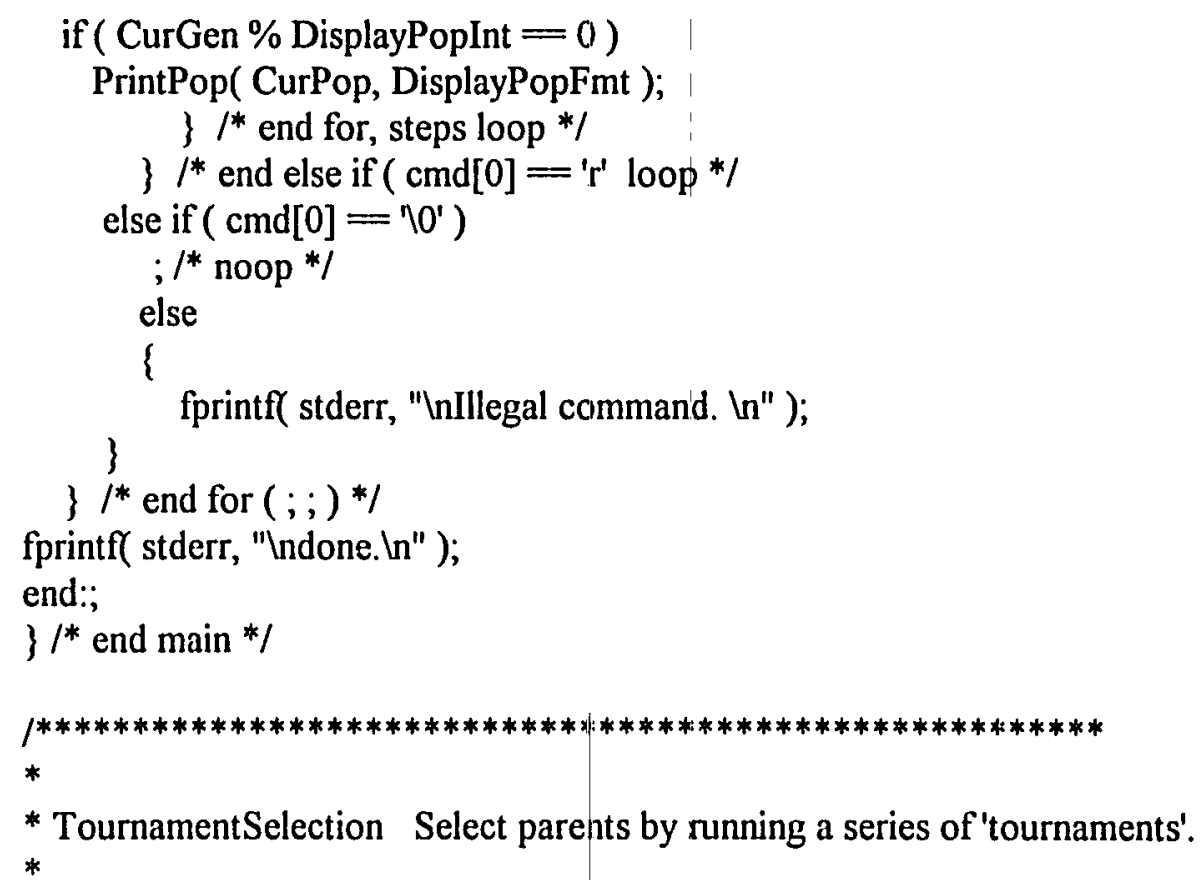


* Pick pairs from the population, and for each pair, pick the highest

* fitness to be reproduced with probability TSProb (eg 0.75 ),

* otherwise use the lower fitness member of the pair.

* Continue this until the new population is the desired size.

* Pick the pairs (uniform) randomly, with replacement.

* cf. Goldberg and Deb.

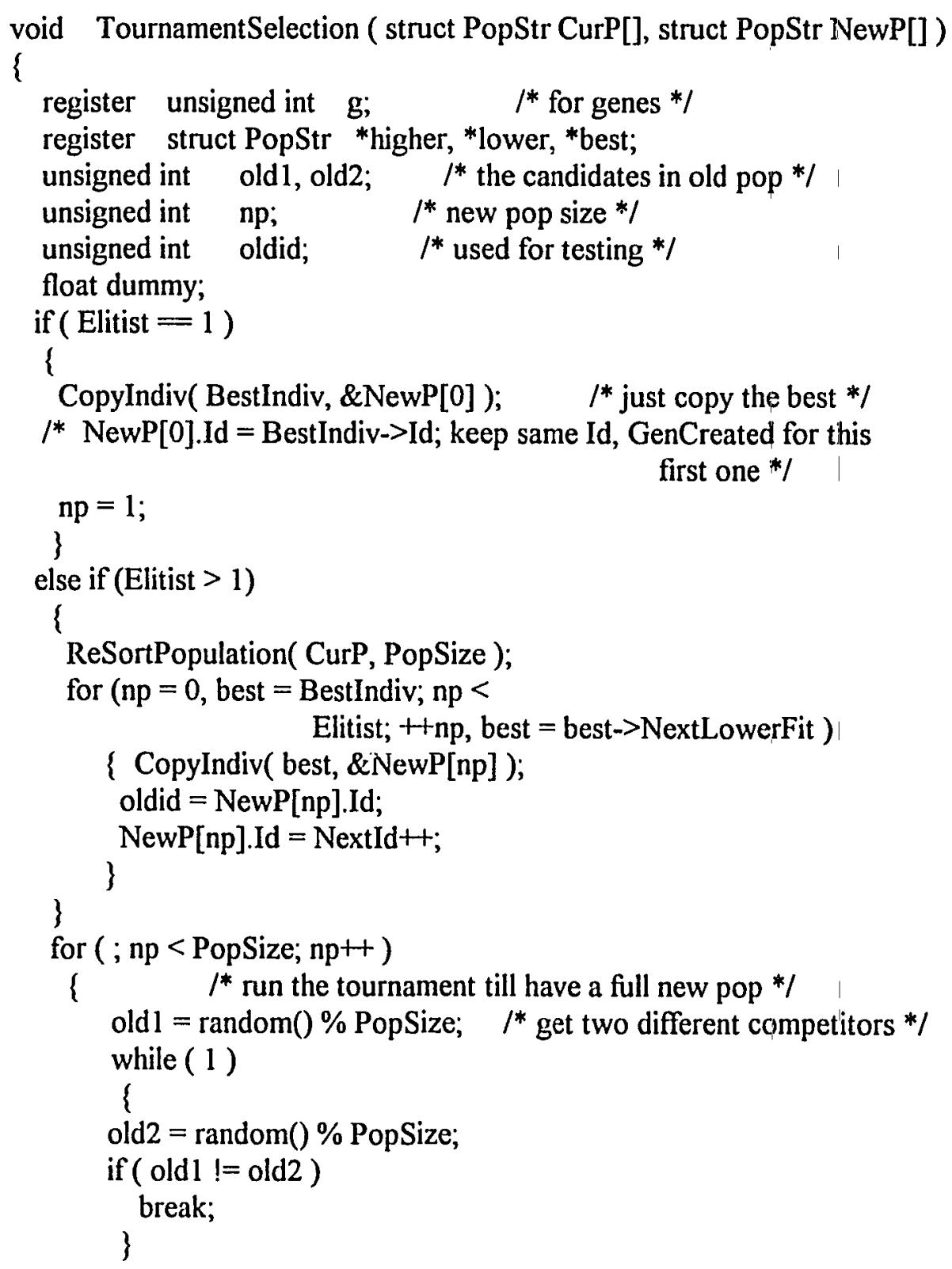


if ( CurP[old1].Fitness $>=$ CurP[old2].Fitness )

$\left\{1^{*}\right.$ figure out which has greater fitness *\}

higher $=\&$ CurP $[$ old 1$]$;

lower $=\&$ CurP[old2];

\}

else

\{

higher $=\&$ CurP $[$ old2];

lower $=\&$ CurP[old1]

)

if ( URand01 < TSProb )

( $/ *$ the best wins! */

CopyIndiv( higher, \&NewP[np] );

oldid $=$ higher $->$ Id;

\}

else

$\left\{l^{*}\right.$ the weaker wins! */

CopyIndiv( lower, \&NewP[np] );

oldid = lower- $>$ Id;

\}

NewP[np].Id = NextId ++;

\}

for $(n p=0 ; n p<$ PopSize; + $n p) \quad / *$ Reassign IDs starting with $1 * /$

NewP[np].Id = np;

BestIndiv->Id = NewP[0].Id;

NextId = PopSize;

\}$/ *$ end TournamentSelection */

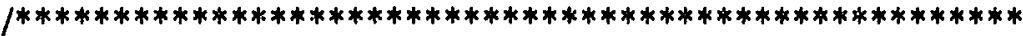

* CopyIndiv Copy an individual's Genome and Fitness from one

* structure to another.

****************************************************/

void CopyIndiv( struct PopStr *From, struct PopStr *To )

\{

register unsigned int $\mathrm{g}$;

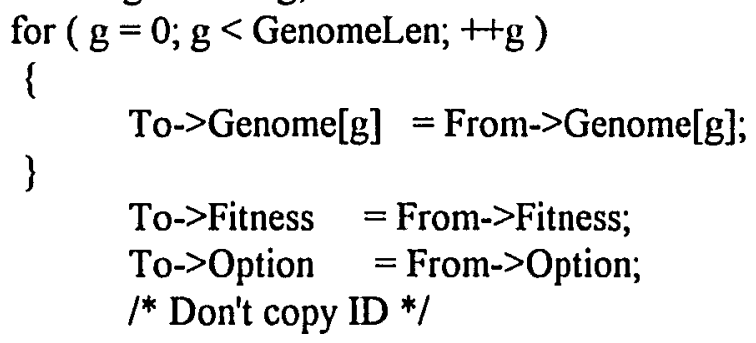




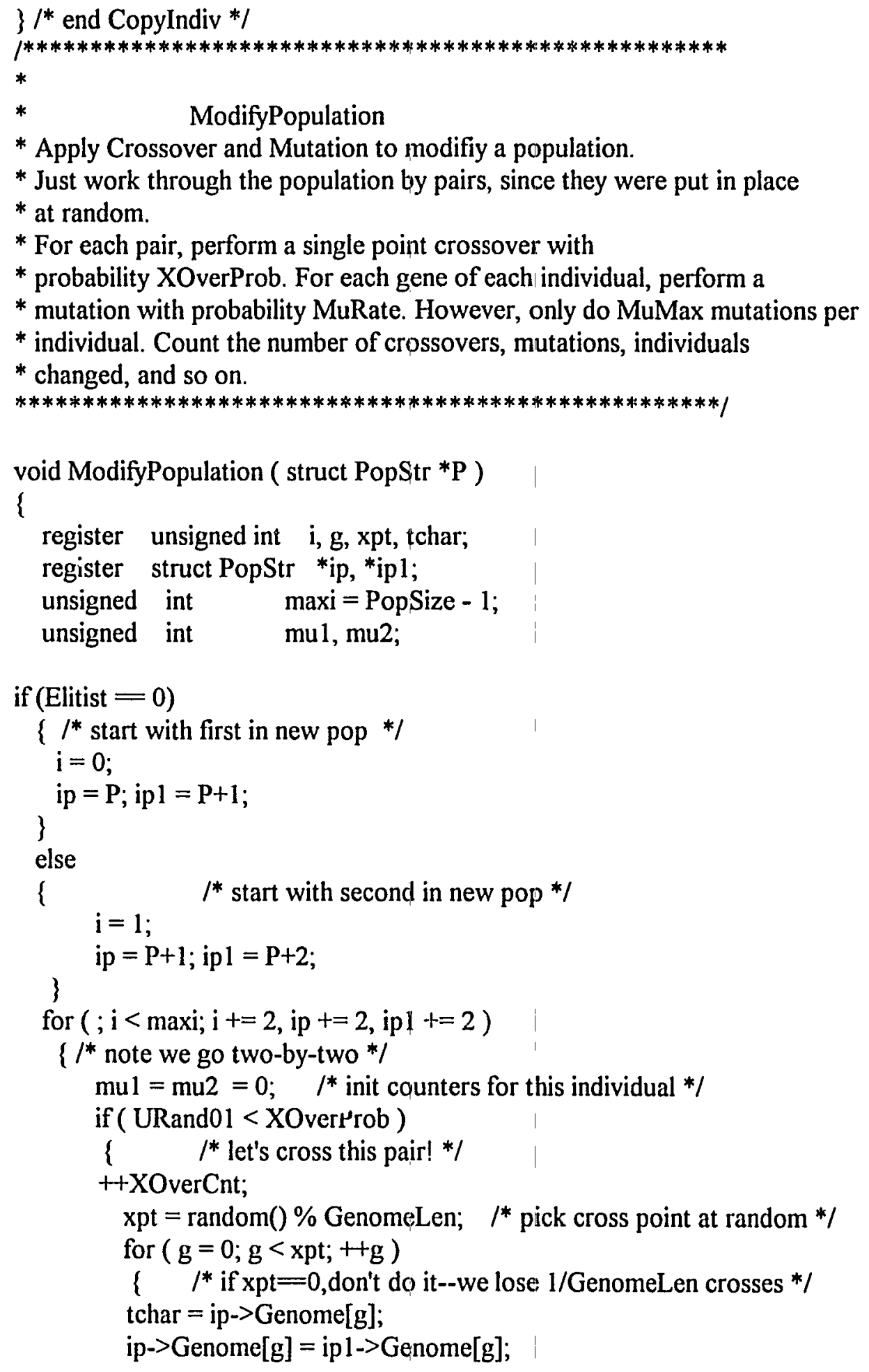




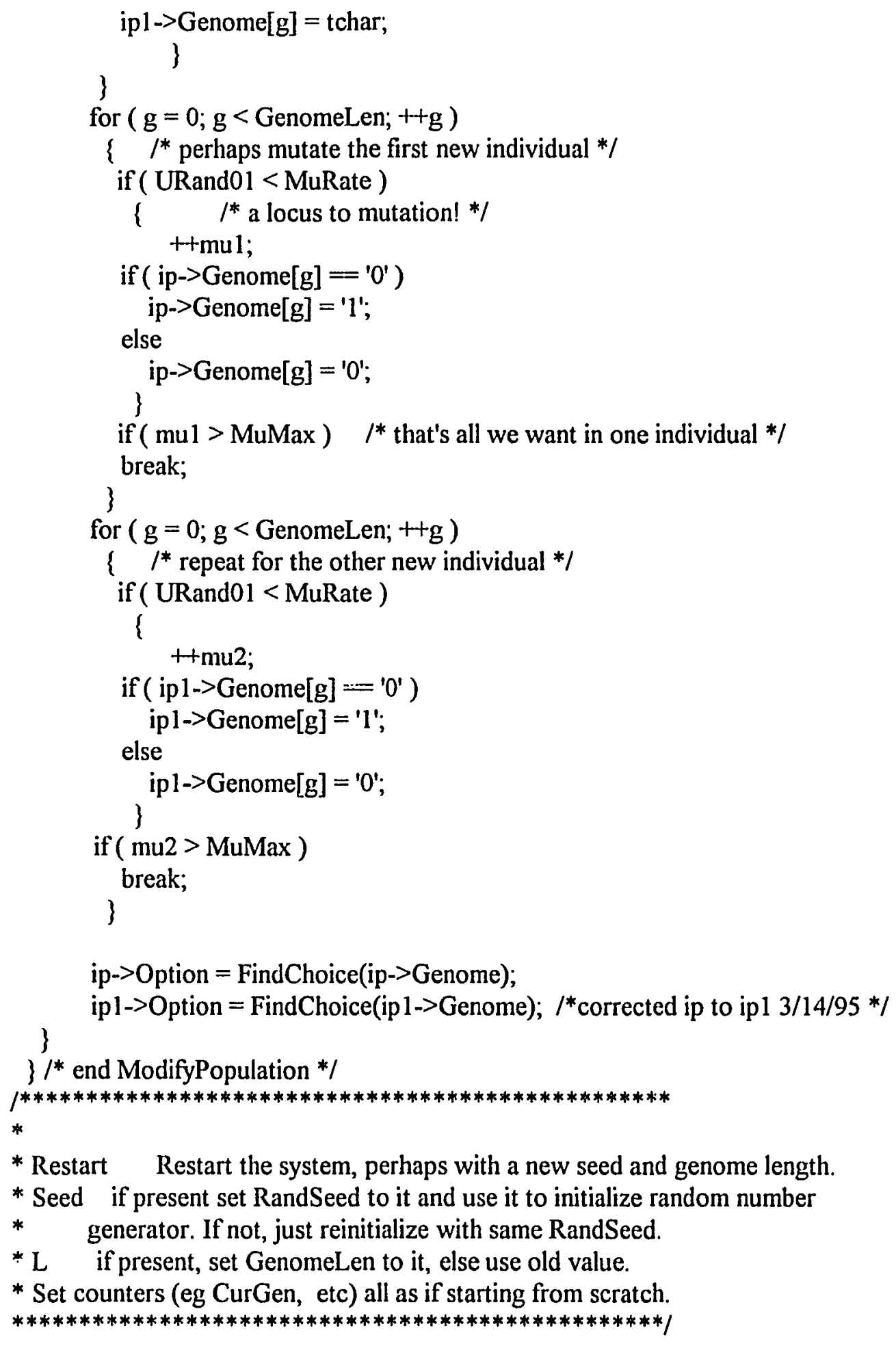




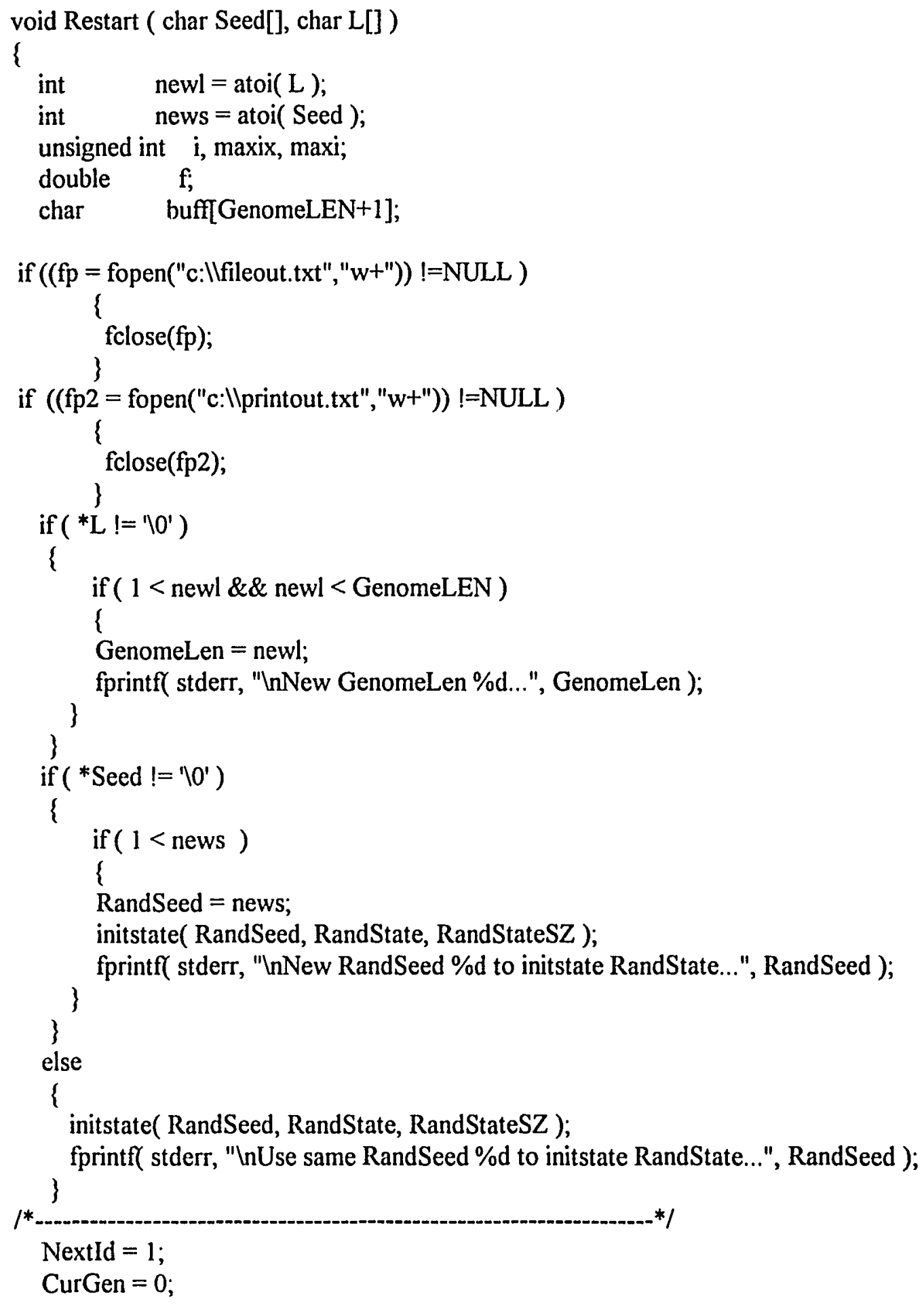




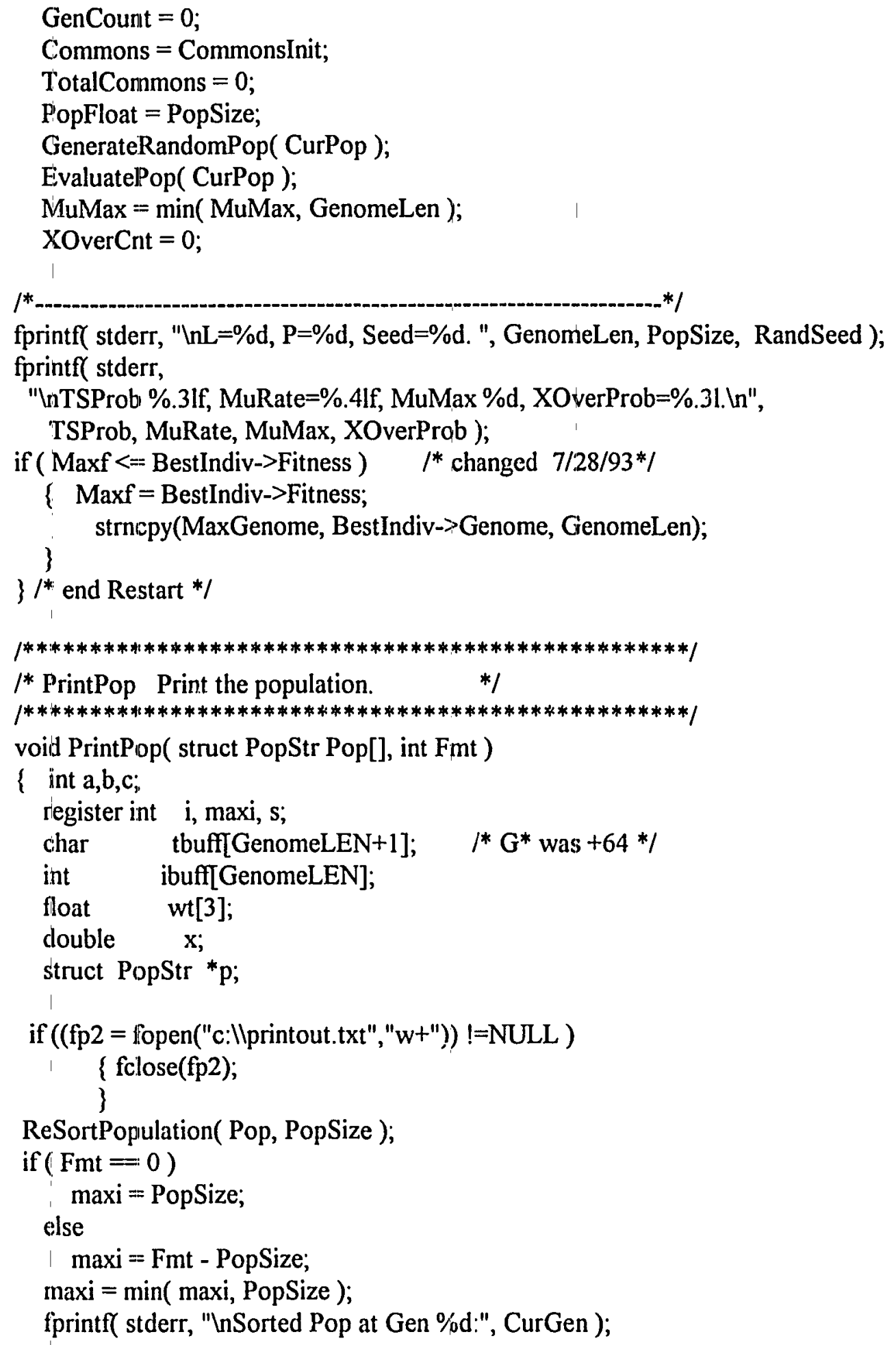




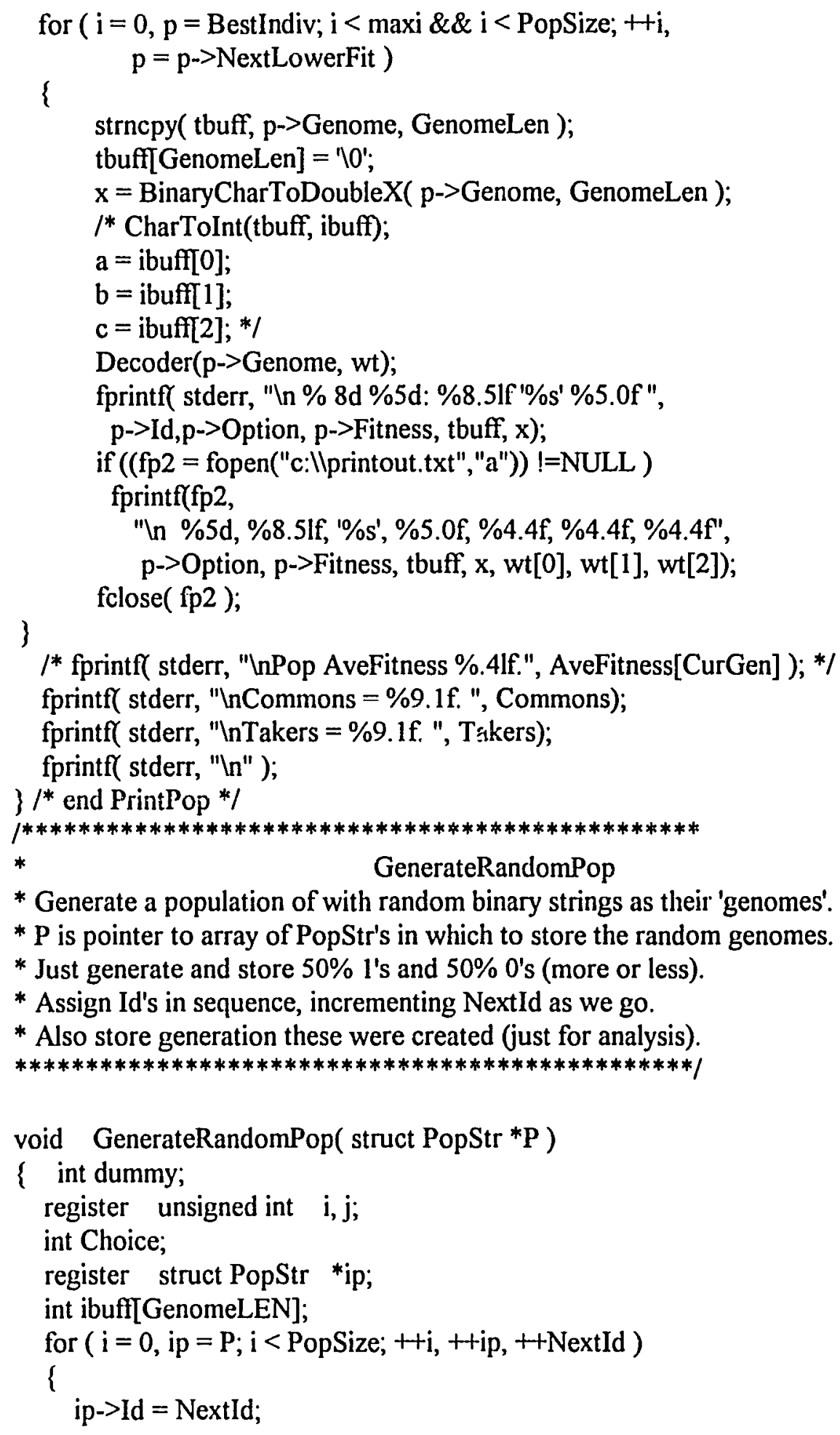

GenerateRandomPop

* Generate a population of with random binary strings as their 'genomes'.

* $\mathrm{P}$ is pointer to array of PopStr's in which to store the random genomes.

* Just generate and store 50\% 1's and 50\% 0's (more or less).

* Assign Id's in sequence, incrementing NextId as we go.

* Also store generation these were created (just for analysis).

$* * * * * * * * * * * * * * * * * * * * * * * * * * * * * * * * * * * * * * * * * * * * * * * * 1$

void GenerateRandomPop( struct PopStr *P)

( int dummy;

register unsigned int $\mathrm{i}, \mathrm{j}$;

int Choice;

register struct PopStr *ip; int ibuff[GenomeLEN];

for $(i=0$, ip = P; $i<$ PopSize; + +i, ++ip, ++Nextld $)$

\{

ip->Id = NextId; 


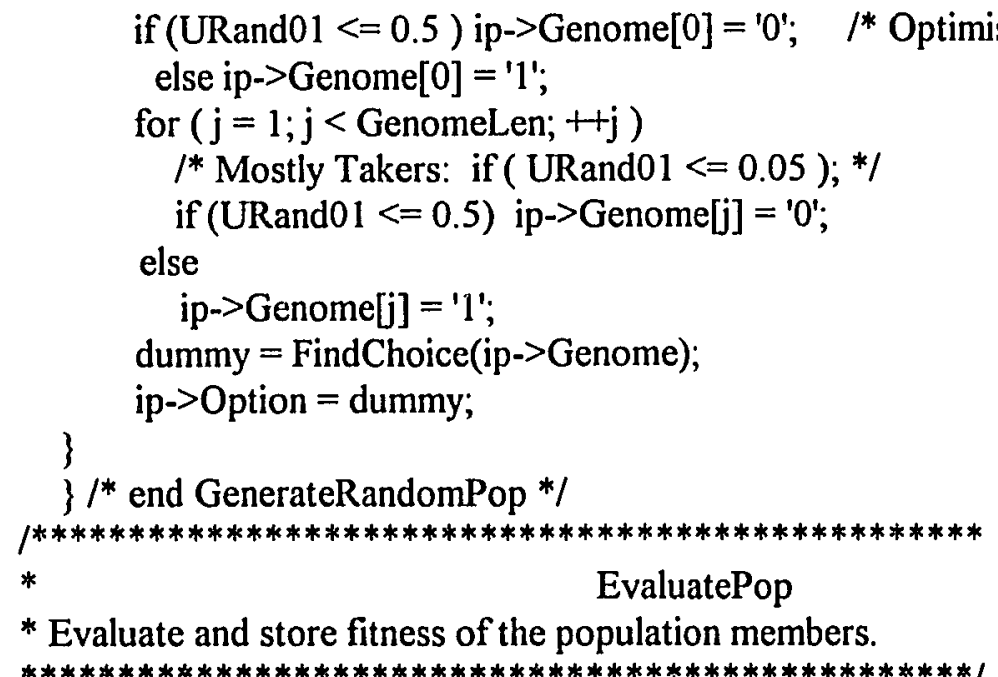

void EvaluatePop( struct PopStr * P )

\{ float w[3];

float $\mathrm{k}, \mathrm{d}$;

float FitnessOfString;

register unsigned int $i$;

register struct PopStr *ip;

register double avef;

int SumOption, Choice, dummy;

BestIndiv $=\mathrm{P} ;$ BestIndiv- $>$ Fitness $=0$;

for $(i=0$, ip $=P$, SumOption $=0 ; i<$ PopSize; ++i, ++ip )

\{ SumOption += ip->Option;

\}

Takers $=$ SumOption;

if(CurGen $=0)$ Commons = CommonsInit;

else

$\{/ *$ Grant's equation, for delta $t=1: * /$

Regen $=$ RegenRate ${ }^{*}$ Commons-(RegenRate/10000)*Commons ${ }^{*}$ Commons; $\mathrm{z}=$ gasdev();

StochReg $=z^{*}($ Range*Regen $)+$ Regen;

Commons $=$ StochReg + Commons - Takers ;

TotalCommons $+=$ Commons;

AveCommons $=$ TotalCommons $/$ GenCount;

TotalTakers $+=$ Takers;

AveTakers $=$ TotalTakers/GenCount; 


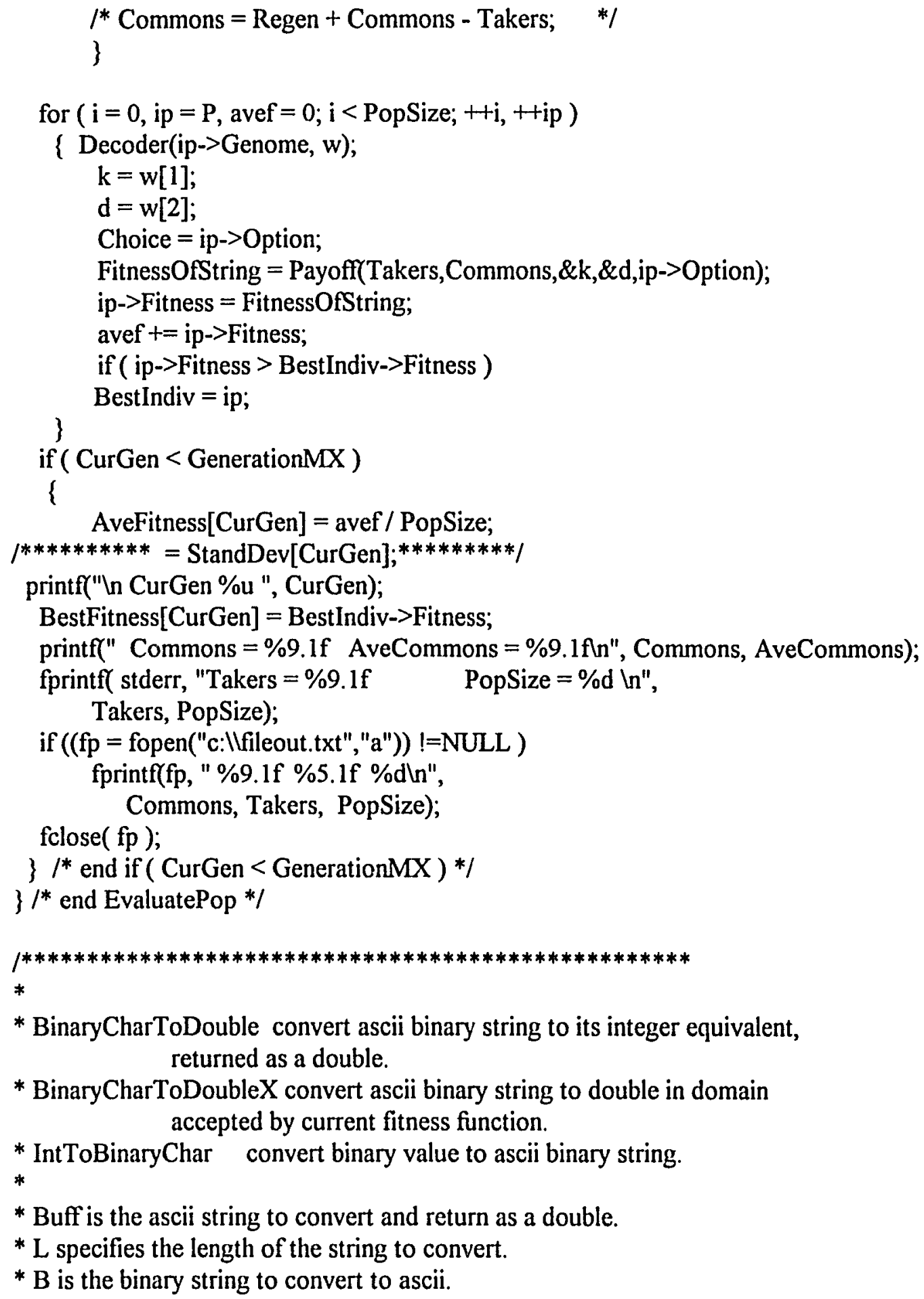

* BinaryCharToDouble convert ascii binary string to its integer equivalent, returned as a double.

* BinaryCharToDoubleX convert ascii binary string to double in domain accepted by current fitness function.

* IntToBinaryChar convert binary value to ascii binary string.

*

* Buff is the ascii string to convert and return as a double.

* L specifies the length of the string to convert.

${ }^{*} \mathrm{~B}$ is the binary string to convert to ascii. 
* BinaryCharToDouble maps an ascii binary representation into a double (real),

* where $000 \ldots 00$ maps to $0,000 \ldots 01$ to $1, \ldots$ etc., up to $111 \ldots 11$ to $2^{* * *} \mathrm{~L}-1$.

* IntToBinaryChar does the inverse.

* BinaryChar2DoubleX just calls BinaryCharToDouble, and then scales the return * value so its in the domain of the current fitness function.

*

$\mid * * * * * * * * * * * * * * * * * * * * * * * * * * * * * * * * * * * * * * * * * * * * * * * * /$

double BinaryCharToDouble ( register char *Buff, register int L )

\{

register int $\mathrm{i}, \mathrm{r}$;

for $(i=r=0 ; i<L ;++i,+$ Buff $)$

$\{\mathrm{r}<<=1$;

if $\left({ }^{*} \mathrm{Buff}={ }^{\prime} 1^{\prime}\right)++\mathrm{r}$;

\}

return( (double) $\mathrm{r}$ );

\} /* end BinaryCharToDouble */

/**************************************************/

double BinaryCharToDoubleX( char *Buff, int L)

\{

double $\mathrm{x}=$ BinaryCharToDouble $($ Buff, $\mathrm{L})$;

\} /* end BinaryCharToDoubleX *I

/***********************************************/

int IntToBinaryChar ( int B, char *Buff, int L )

\{

register int $\mathrm{i}$;

for $(i=L-1 ; i>=0 ;--i)$

$($ if $(B \& 1)$

Buff[i] = ' 1 ';

else

Buff[i] = '0';

$\mathrm{B} \gg=1$;

\}

\}/* end Int ToBinaryChar */

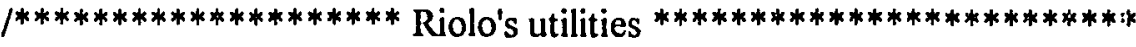

*

* Included here are:

* - routines to count schema instances, calculate average schema fitnesses,

* and so on

* - routines to sort the population by fitness

* - routines to read lines and help parsing them. 
* - routines to set and display various parameters

* - routines to allow the user to see the ascii binary string representations

* of specified decimal values, and to see the fitness assigned by the current fitness function.

* (and to do the inverse, i.e., display the decimal versiorr of an ascii string).

*

$* * * * * * * * * * * * * * * * * * * * * * * * * * * * * * * * * * * * * * * * * * * * * * * * * * * * * * * * /$

/************************************************

*

ReSortPopulation

* This just uses InsertSortIndiv() to re-insert sort individuals, since the

* list is typically almost sorted anyway (i.e., most individuals don't change

* relative fitness if we turnover only a small fraction of the population

* each step).

* For efficiency, start with the high-fitness end, since InsertSortIndiv0

* inserts at low end.

* NOTE:

* On loading individuals (either vi LoadPop or GenRandPop), HighFitIndiv

* and LowFitIndiv list heads are NULLed, and then InsertSortIndiv() is

* called to set up the list.

*

* The Individual nodes are linked to form a two-way linked list sorted on

* fitness.

* HighFitIndiv points to the highest, and NextLowerFit is link to decreasing

* fitness individuals.

* LowFitIndiv points to lowest fit, and NextHigherFit is link to increasing

* fitness.

*

*****************************************/

int ReSortPopulation ( struct PopStr Pop[], unsigned int Size )

\{ unsigned int $\mathrm{i}$;

BestIndiv $=$ WorstIndiv $=$ NULL;

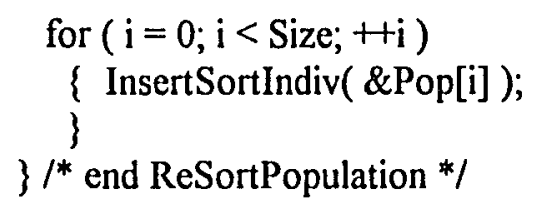

int InsertSortIndiv( struct PopStr *IPtr )

\{ double f; 


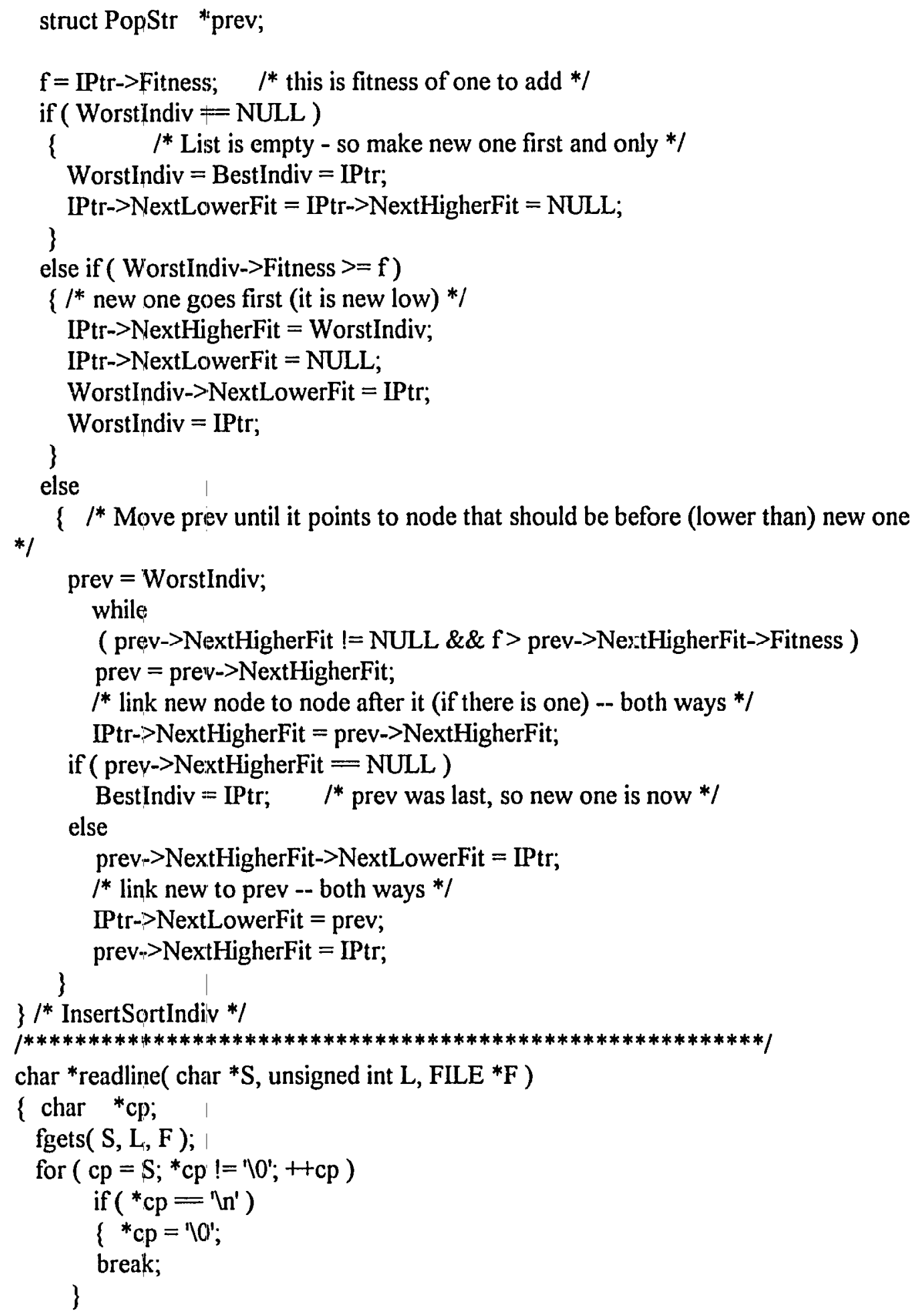




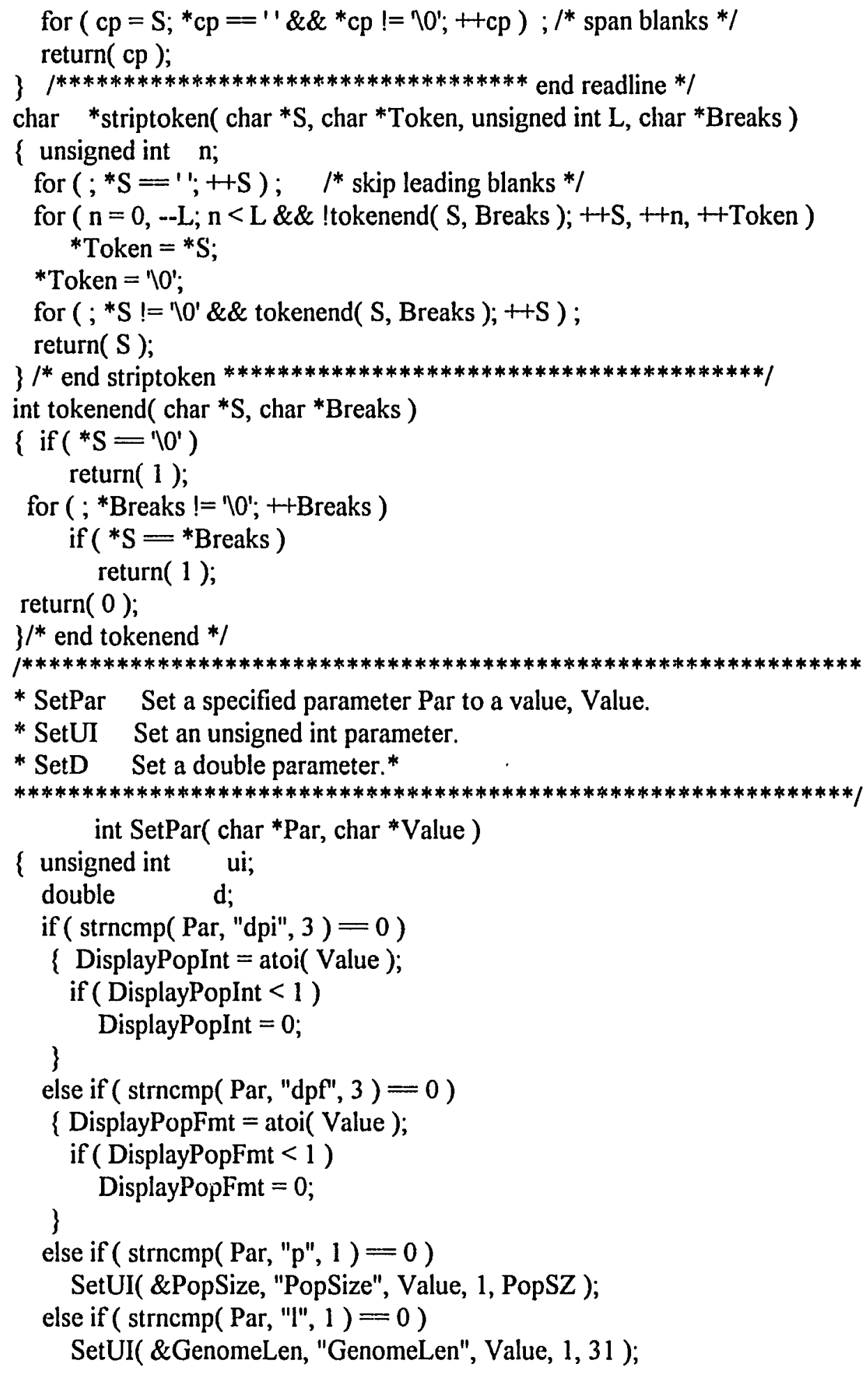




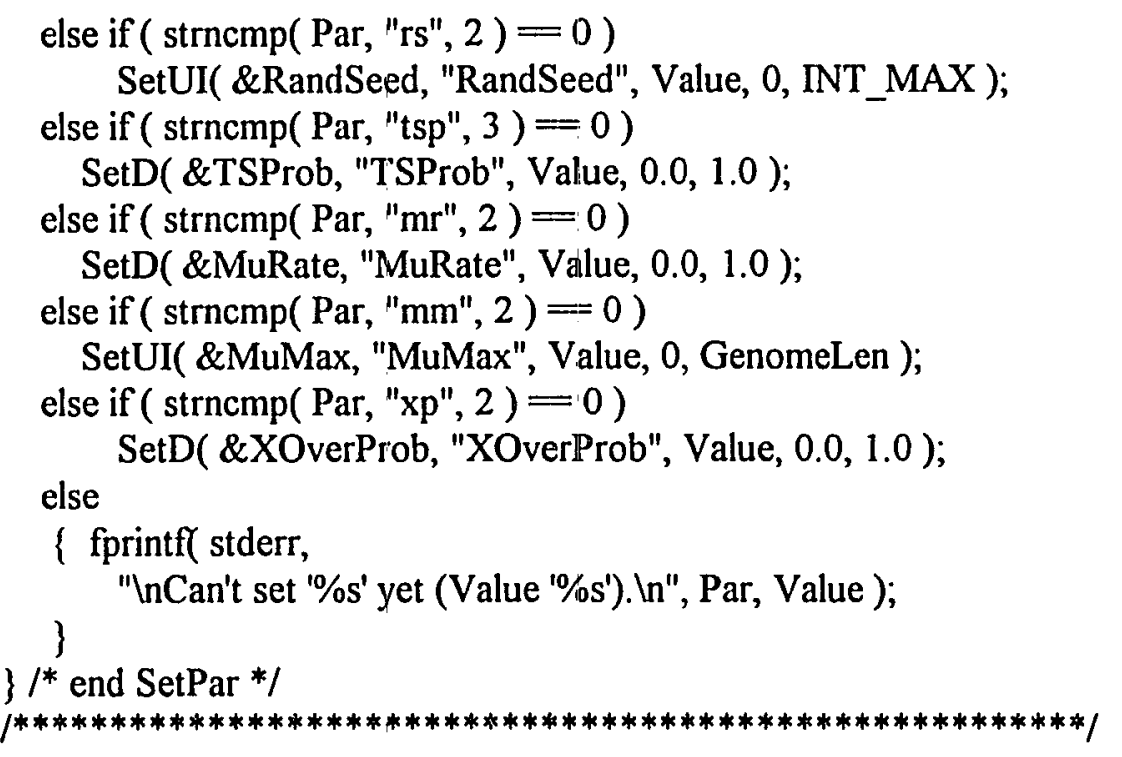

int SetUI( unsigned int *Var, char *VarName, char *Val, unsigned int LB, unsigned int UB )

\{

unsigned int ui;

ui = atoi( Val $)$;

if ( ui < LB $\|$ ui > UB )

fprintf( stderr, "InIllegal \%s '\%s' (LB \%d, UB \%d: ui=\%d). In", VarName, Val,

LB, UB, ui );

else

*Var = ui;

\}/* end SetUI *I

int SetD( double *Var, char *VarName, char *Val, double LB, double UB )

\{

double d;

$\mathrm{d}=$ atof $(\mathrm{Val})$;

if $(\mathrm{d}<\mathrm{LB} \| \mathrm{d}>\mathrm{UB})$

fprintf( stderr, "Inlllegal \%s '\%s' (LB \%lf, UB \%lf: $d=\%$ lf).In", VarName, Val,

LB, UB, d);

else

$* \operatorname{Var}=\mathrm{d}$;

\}$/ *$ end SetD */ 


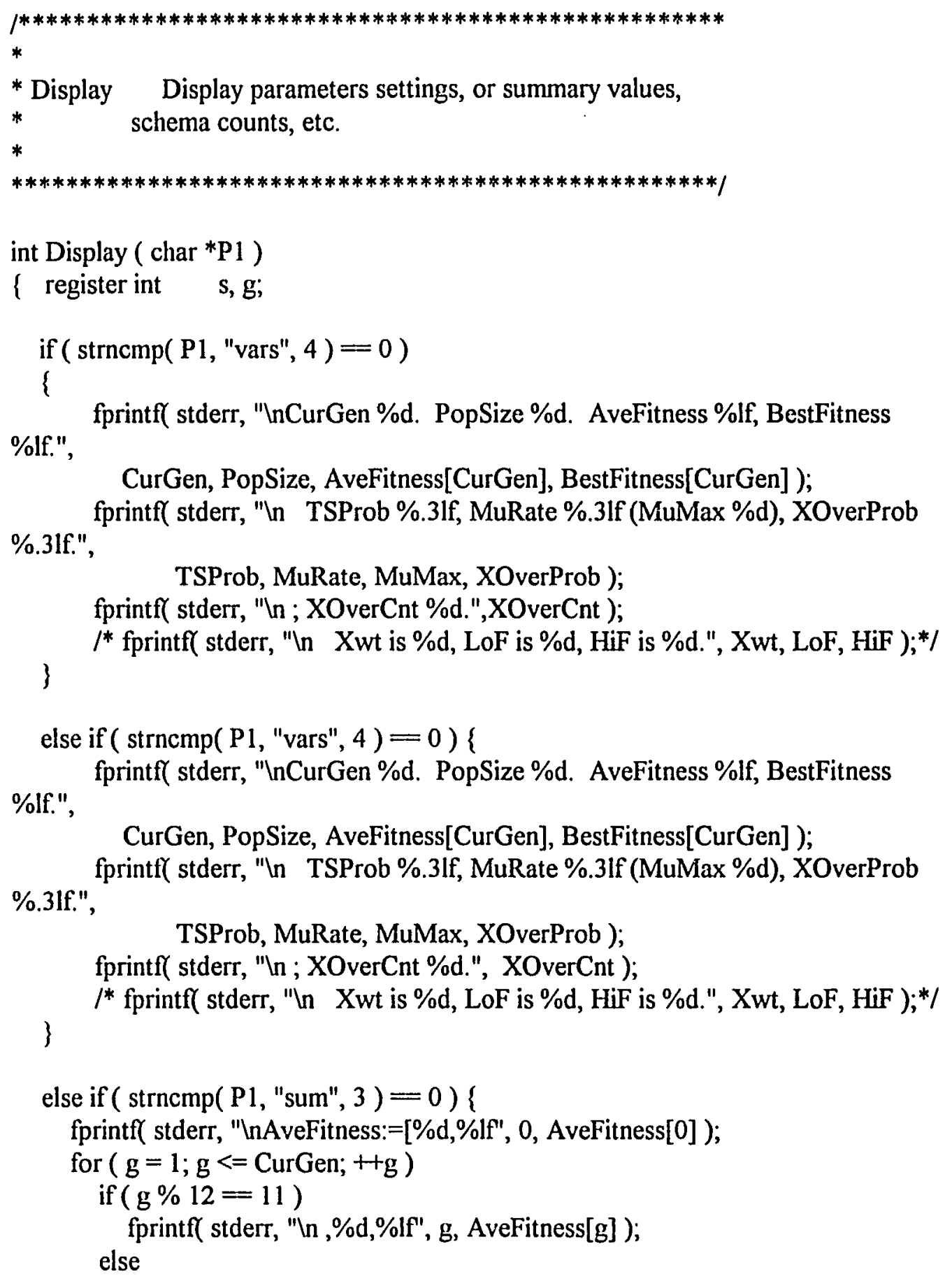




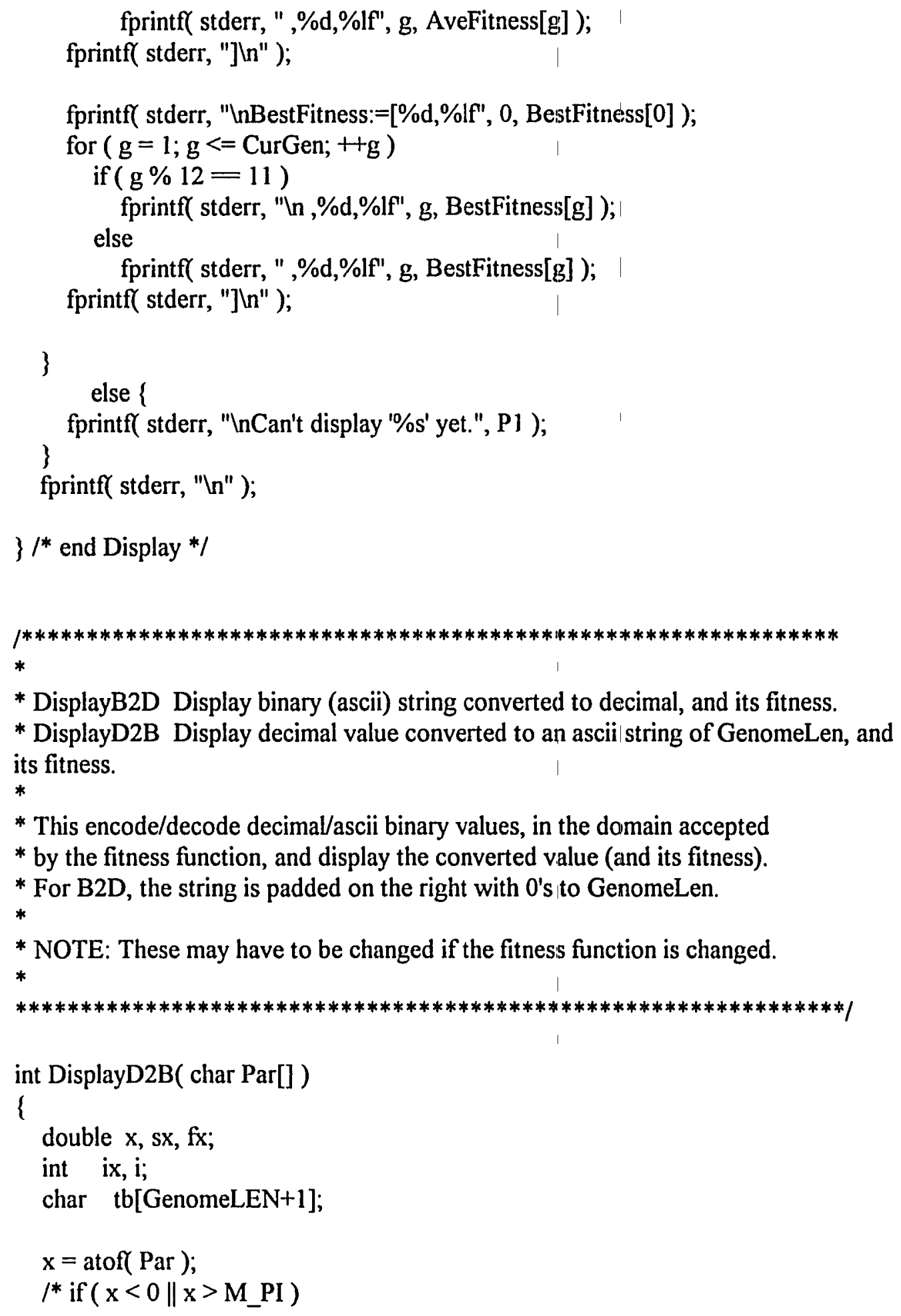

* This encode/decode decimal/ascii binary values, in the domain accepted

* by the fitness function, and display the converted value (and its fitness).

* For B2D, the string is padded on the right with 0 's to GenomeLen.

*

* NOTE: These may have to be changed if the fitnesis function is changed.

*

$* * * * * * * * * * * * * * * * * * * * * * * * * * * * * * * * * * * * * * * * * * * * * * * * * * * * * * * * * * * * * * * * 1$

int DisplayD2B( char Par[])

l

double $\mathrm{x}, \mathrm{sx}, \mathrm{fx}$;

int ix, $\mathrm{i}$;

char tb[GenomeLEN+1];

$\mathrm{x}=$ atof( $\mathrm{Par})$;

$l^{*}$ if $(x<0 \| x>$ M_PI $)$ 

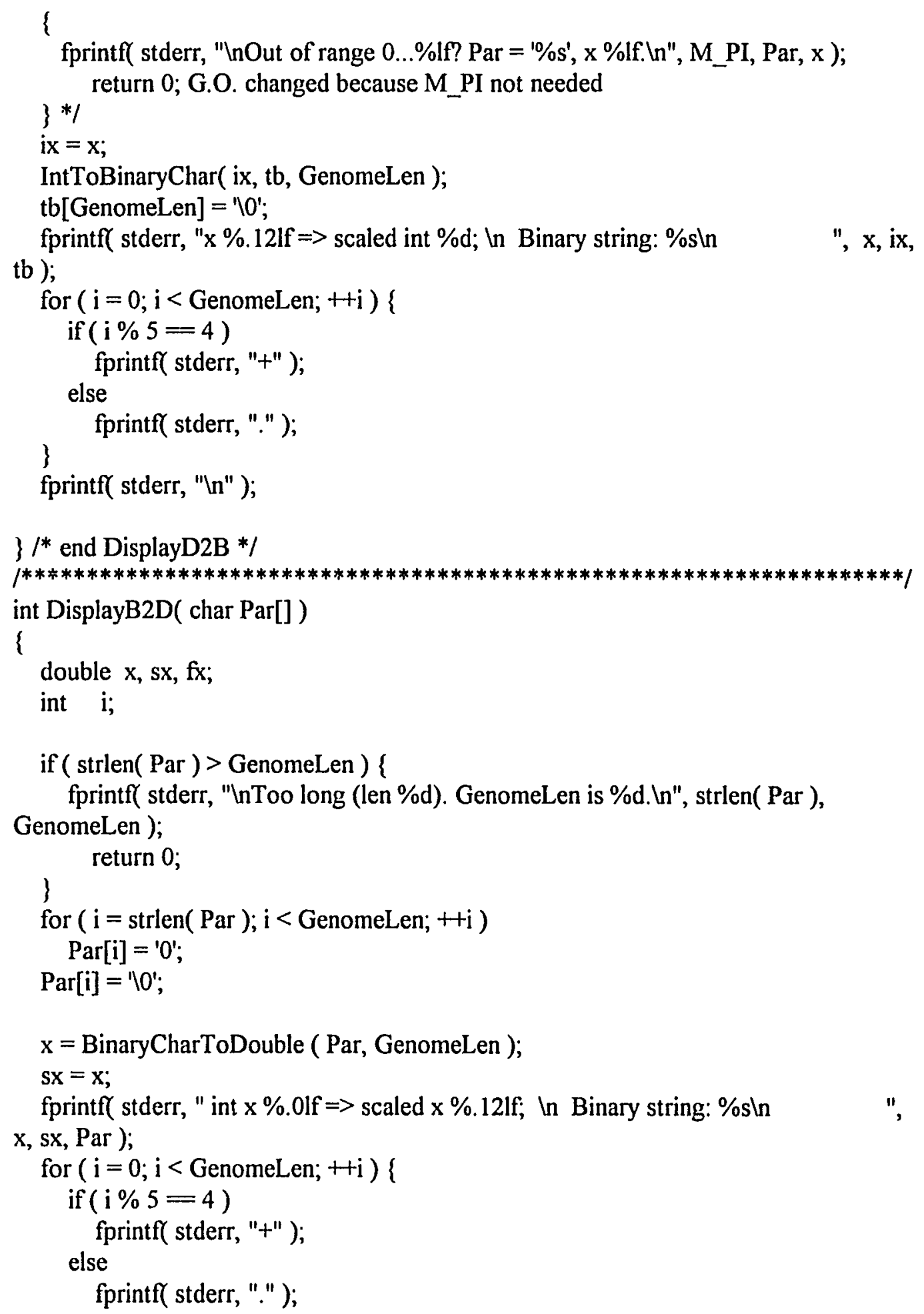


\author{
\} \\ fprintf( stderr, "In" ); \\ \} /* end DisplayB2D */
}

\title{
/* TOC FUNCTIONS */
}

void CharToInt(char Charray[4], int Intarray[3])

\{ char buf[2];

int $\mathrm{i}$;

int dummy;

for $(\mathrm{i}=0$; $\mathrm{i}<$ GenomeLen; $\mathrm{i}++)$

\{

buf $[0]=$ Charray $[\mathrm{i}]$;

dummy = atoi(buf);

\}

Intarray [i] = dummy;

\} /*end of CharToInt*/

\section{/* DECODER */}

void Decoder(char Genotype[4], float w[3])

( char a,b,c;

$\mathrm{a}=$ Genotype[0];

$\mathrm{b}=$ Genotype[1];

c = Genotype[2];

if (Genotype[0] $={ }^{\prime} 0$ ') $w[0]=0$;

$$
\text { else } w[0]=1 ; \quad \quad / * \text { Optimist */ }
$$

if (Genotype[1] == '0'\&\& Genotype[2] = '0')

$\{w[1]=0 ; w[2]=0 ;\}$

if (Genotype[1] = '0' \& \& Genotype[2] $=$ '1')

$\{w[1]=0 ; w[2]=1 ;\}$

if (Genotype[1] = ' 1 ' \& \& Genotype[2] ='0')

$\{w[1]=1 ; w[2]=0 ;\}$

if (Genotype[1] = ' 1 ' \&\& Genotype[2] = '1')

$\{w[1]=1 ; w[2]=1 ;\}$

$I^{*} w[0]=1 ; w[1]=0 ; w[2]=1 ; * /$

\}

/* FINDCHOICE *

int FindChoice(char geno[]) 
\{ float w[3], k, d, Opt, PessTakers, OptTakers;

float PrRight $=.75$;

float TakeSum = 0; float RefrainSum =0;

int Take;

float GoodCommons, BadCommons;

Decoder(geno, w);

Opt $=w[0] ; k=w[1] ; d=w[2] ;$

PessTakers $=$ PopSize; if $($ PessTakers $>$ PopSize $)$ PessTakers $=$ PopSize;

OptTakers $=.5 *$ PopSize; $\quad / * .5$ times, $* /$

BadCommons $=0.7^{*}$ AveCommons; if(BadCommons $\left.<0\right)$ BadCommons $=0$;

GoodCommons $=1.5 *$ AveCommons ; if $($ GoodCommons $>10000)$ GoodCommons $=$ 10000 ;

if $(\mathrm{Opt}==0)$

$\{$ TakeSum $=$ PrRight* (Payoff(PessTakers, BadCommons, \&k, \&d, 1));

TakeSum += (1-PrRight)* (Payoff(OptTakers, GoodCommons, \&k, \&d, 1));

RefrainSum $=$ PrRight $*($ Payoff(PessTakers, DadCommons, \&k, \&d, 0));

RefrainSum $+=(1-P r R i g h t) *$ (Payoff(OptTakers, GoodCommons, \&k, \&d, 0));

\}

else

$\{$ TakeSum $=$ PrRight $*($ Payoff(OptTakers, GoodCommons , \&k, \&d, 1));

TakeSum $+=(1-P r R i g h t) *$ (Payoff(PessTakers, BadCommons, \&k, \&d, 1));

RefrainSum $=$ PrRight * (Payoff(OptTakers, GoodCommons, \&k, \&d, 0));

RefrainSum +=(1-PrRight) * (Payoff(PessTakers, BadCommons, \&k, \&d, 0)); \}

if(TakeSum $<$ RefrainSum) Take $=0$;

else Take = 1 ;

return(Take);

\}

/* PAYOFF */

float Payoff(float NTakers, float State, float *k, float ${ }^{*} d$, int Choice)

\{ float fpayoff, b,m,kk,dd, Conscience;

$\mathrm{b}=1.5 ; /^{*}$ profit function parameter */

$\mathrm{kk}=* \mathrm{k}$;

$\mathrm{dd}=* \mathrm{~d}$;

$\mathrm{m}=0.03 ; l^{*} \mathrm{C}(\mathrm{x})$ parameter ${ }^{*}$

Conscience $=-\mathrm{m}^{*}$ State $+\mathrm{m} * 10000$

if $(\mathrm{kk}=0 \& \& \mathrm{dd}=0) \quad / *$ type $0,4 * /$

$\{$ if(Choice $=0)$ fpayoff $=0$;

else fpayoff $=b^{*}($ PopSize-NTakers $)$;

\}

else if $(k \mathrm{k}==0$ \&\& $\mathrm{dd}>0) \quad \quad I^{*}$ type $1,5 * /$ 


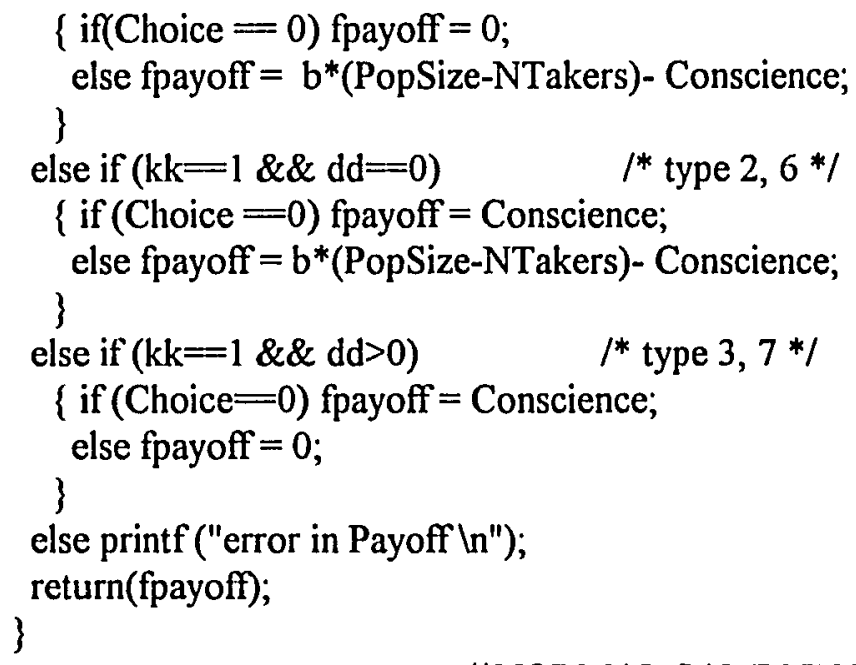

/*NORMAL SAMPLING */

float gasdev()

$\{$ static int iset $=0$;

static float gset;

float fac, rsq, v1, v2;

if (iset $=0$ )

$\{$ do

$\{v\}=2.0 *$ URand01-1.0;

$\mathrm{v} 2=2.0 *$ URand01-1.0;

\}

$\mathrm{rsq}=\mathrm{v} 1 * \mathrm{v} 1+\mathrm{v} 2 * \mathrm{v} 2$;

whila $(\mathrm{rsq}>=1.0 \| \mathrm{rsq}==0.0)$;

fac $=\operatorname{sqrt}\left(-2.0^{*} \log (\mathrm{rsq}) / \mathrm{rsq}\right)$;

gset=vl*fac;

iset $=1$;

return v2*fac;

\}

else

$\{$ iset $=0$;

return gset;

) 
if (URand01 $<=0.5)$ CurP[I].Genome[j] = ' 0 '; else CurP[I].Genome[j] = ' 1 ';

CurP[I].Option = FindChoice(CurP[I].Genome);

CurP[I].Id = NextId; \} 